EPJ manuscript No.

(will be inserted by the editor)

\title{
Physics with the KLOE-2 experiment at the upgraded DAФNE
}

\begin{abstract}
G. Amelino-Camelia ${ }^{1,2}$, F. Archilli ${ }^{3,4}$, D. Babusci ${ }^{5}$, D. Badoni ${ }^{4}$, G. Bencivenni ${ }^{5}$, J. Bernabeu ${ }^{6}$, R.A. Bertlmann ${ }^{7}$, D.R. Boito ${ }^{8}$, C. Bini ${ }^{1,2}$, C. Bloise ${ }^{5}$, V. Bocci ${ }^{2}$, F. Bossi ${ }^{5}$, P. Branchini ${ }^{9}$, A. Budano ${ }^{9}$, S.A. Bulychjev ${ }^{10}$, P. Campana ${ }^{5}$,

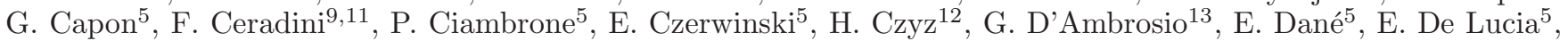
G. De Robertis ${ }^{14}$, A. De Santis ${ }^{1,2}$, P. De Simone ${ }^{5}$, G. De Zorzi ${ }^{1,2}$, A. Di Domenico ${ }^{1,2}$, C. Di Donato ${ }^{13}$, B. Di Micco 9,11 a , D. Domenici ${ }^{5}$, S.I. Eidelman ${ }^{15}$, O. Erriquez ${ }^{14,16}$, R. Escribano ${ }^{8}$, R. Essig17 ${ }^{17}$, G.V. Fedotovich ${ }^{15}$, G. Felici ${ }^{5}$, S. Fiore ${ }^{1,2}$, P. Franzini ${ }^{1,2}$, P. Gauzzi ${ }^{1,2}$, F. Giacosa ${ }^{18}$, S. Giovannella ${ }^{5}$, F. Gonnella ${ }^{3,4}$, E. Graziani ${ }^{9}$, F. Happacher ${ }^{5}$, B.C. Hiesmayr ${ }^{7,19}$, B. Höistad ${ }^{20}$, E. $\operatorname{Iarocci}^{5,21}$, S. Ivashyn ${ }^{12,22}$, M. Jacewicz ${ }^{5}$, F. Jegerlehner ${ }^{23}$, T. Johansson ${ }^{20}$, J. Lee-Franzini ${ }^{5}$, W. Kluge ${ }^{24}$, V.V. Kulikov ${ }^{10}$, A. Kupsc ${ }^{20}$, R. Lehnert ${ }^{25}$, F. Loddo ${ }^{14}$, P. Lukin $^{15}$, M.A. Martemianov ${ }^{10}$, M. Martini ${ }^{5,21}$, M.A. Matsyuk ${ }^{10}$, N.E. Mavromatos ${ }^{26}$, F. Mescia ${ }^{27}$, R. Messi ${ }^{3,4}$, S. Miscetti ${ }^{5}$, G. Morello ${ }^{28,29}$, D. Moricciani ${ }^{4}$, P. Moskal ${ }^{30}$, S. Müller ${ }^{5 b}$, F. Nguyen ${ }^{9}$, E. Passemar $^{6,31}$, M. Passera ${ }^{32}$, A. Passeri ${ }^{9}$, V. Patera ${ }^{5,21}$, M.R. Pennington ${ }^{33}$, J. Prades $^{34}$, L. Quintieri ${ }^{5}$, A. Ranieri ${ }^{14}$, M. Reece ${ }^{35}$, P. Santangelo ${ }^{5}$, S. Sarkar ${ }^{26}$, I. Sarra ${ }^{5}$, M. Schioppa ${ }^{28,29}$, P.C. Schuster ${ }^{17}$, B. Sciascia ${ }^{5}$, A. Sciubba ${ }^{5,21}$, M. Silarski ${ }^{30}$, C. Taccini ${ }^{9,11}$, N. Toro ${ }^{36}$, L. Tortora ${ }^{9}$, G. Venanzoni ${ }^{5}$, R. Versaci ${ }^{5 a}$, L.-T. Wang ${ }^{37}$, W. Wislicki ${ }^{38}$, M. Wolke ${ }^{20}$, and J. Zdebik ${ }^{30}$
\end{abstract}

1 Dipartimento di Fisica, Università "Sapienza", Roma, Italy

2 INFN, Sezione di Roma, Roma, Italy

3 Dipartimento di Fisica, Università "Tor Vergata", Roma, Italy

4 INFN, Sezione Roma 2, Roma, Italy

5 INFN, Laboratori Nazionali di Frascati, Frascati, Italy

6 Departamento de Fìsica Teòrica and IFIC, Universidad de Valencia-CSIC, Valencia, Spain

7 University of Vienna, Vienna, Austria

${ }^{8}$ Grup de Fìsica Teòrica and IFAE, Universitat Autònoma de Barcelona, Spain

9 INFN, Sezione Roma 3, Roma, Italy

10 Institute for Theoretical and Experimental Physics, Moscow, Russia

11 Dipartimento di Fisica, Università "Roma Tre", Roma, Italy

12 Institute of Physics, University of Silesia, Katowice, Poland

13 INFN, Sezione di Napoli, Napoli, Italy

14 INFN, Sezione di Bari, Bari, Italy

15 Budker Institute of Nuclear Physics, Novosibirsk, Russia

16 Dipartimento di Fisica, Università di Bari, Bari, Italy

17 SLAC National Accelerator Laboratory, Menlo Park, U.S.A.

18 Institute for Theoretical Physics, Goethe University, Frankfurt, Germany

19 University of Sofia, Sofia, Bulgaria

20 University of Uppsala, Uppsala, Sweden

21 Dipartimento di Energetica, Università "Sapienza", Roma, Italy

22 Institute for Theoretical Physics, NSC "Kharkov Institute of Physics and Technology", Kharkov, Ukraine

23 Humboldt-Universität zu Berlin, Institut für Physik, Berlin, Germany

24 Institut für Experimentelle Kernphysik, Universität Karlsruhe, Karlsruhe, Germany

${ }^{25}$ Instituto de Ciencias Nucleares, Universidad Nacional Autònoma de México, México D.F., México

26 Department of Physics, King's College, London, England

27 Universitat de Barcelona, Departament ECM and ICC, Barcelona, Spain

28 Università della Calabria, Cosenza, Italy

29 INFN, Gruppo collegato di Cosenza, Cosenza, Italy

30 Jagiellonian University, Cracow, Poland

31 Albert Einstein Center for Fundamental Physics, University of Bern, Bern, Switzerland

32 INFN, Sezione di Padova, Padova, Italy

33 Institute for Particle Physics Phenomenology, Durham University, Durham, U.K.

34 CAFPE and Departamento de Física Teórica y del Cosmos, Universidad de Granada, Granada, Spain

35 Princeton Center for Theoretical Science, Princeton University, Princeton, U.S.A.

\footnotetext{
a Present address: CERN, Geneve, Switzerland

b Present address: Institut für Kernphysik, Gutenberg Universität, Mainz, Germany
} 
36 Stanford Institute for Theoretical Physics, Stanford University, Stanford, U.S.A.

37 Department of Physics, Princeton University, Princeton, U.S.A.

38 A. Soltan Institute for Nuclear Studies, Warsaw, Poland

Received: date / Revised version: date

Abstract. Investigation at a $\phi$-factory can shed light on several debated issues in particle physics. We discuss: i) recent theoretical development and experimental progress in kaon physics relevant for the Standard Model tests in the flavor sector, ii) the sensitivity we can reach in probing CPT and Quantum Mechanics from time evolution of entangled kaon states, iii) the interest for improving on the present measurements of non-leptonic and radiative decays of kaons and $\eta / \eta$ ' mesons, iv) the contribution to understand the nature of light scalar mesons, and v) the opportunity to search for narrow di-lepton resonances suggested by recent models proposing a hidden dark-matter sector. We also report on the $e^{+} e^{-}$physics in the continuum with the measurements of (multi)hadronic cross sections and the study of $\gamma \gamma$ processes. 


\section{Contents}

1 Introduction . . . . . . . . . . . . . .

2 CKM Unitarity and Lepton Universality . . . . . .

2.1 Determination of $f_{+}(0)$ and $f_{K} / f_{\pi} \ldots \ldots$

2.2 Experimental results from kaon decays . . . . .

2.2.1 Branching ratios . . . . . . . . . .

2.2.2 Kaon lifetimes . . . . . . . . . . . . . . .

2.2 .3 Kaon form factors . . . . . . . . . . .

2.2.4 Kaon form factors from $\tau$ decays . . . .

2.2.5 Unitarity and universality . . . . . . . .

2.2.6 $K_{\ell 2}$ and $K_{\ell 3}$ beyond the SM . . . .

2.2.7 Test of lepton-flavor conservation: $R_{K}$.

2.3 KLOE-2 prospects on $\left|V_{u s}\right| \times f_{+}(0) \ldots . .$. .

3 CPT Symmetry and Quantum Mechanics ...... .

3.1 The neutral kaon system at the $\phi$ factory . . .

3.2 Test of quantum coherence . . . . . . . . .

$3.3 C P T$ violation from quantization of space-time

$3.4 C P T$ violation and decoherence . . . . . . .

3.5 CPT violation and Lorentz-symmetry breaking

3.6 Other $C P T$-symmetry tests at the $\phi$ factory . .

3.7 Quantitative Bohr's complementarity . . . . . .

3.7.1 The kaonic quantum eraser . . . . . . .

3.8 KLOE-2 prospects on kaon interferometry . . .

4 Low Energy QCD . . . . . . . . . . . . . . .

4.1 Rare kaon decays . . . . . . . . . . . . . .

$4.1 .1 \quad K \rightarrow 3 \pi \ldots \ldots \ldots \ldots$

4.1.2 $K_{S} \rightarrow \gamma^{(*)} \gamma^{(*)} / K_{S} \rightarrow \pi^{0} \gamma \gamma \ldots \ldots$

4.1.3 $K_{S} \rightarrow \pi^{0} \ell^{+} \ell^{-} \ldots \ldots \ldots \ldots$

4.1.4 $K \rightarrow \pi \pi \gamma / K \rightarrow \pi \pi e e \ldots . . . .$.

4.2 Physics of $\eta$ and $\eta^{\prime}$ mesons . . . . . . . . . .

4.2.1 Hadronic decays into three pions ....

$4.2 .2 \quad \eta / \eta^{\prime} \rightarrow \pi^{+} \pi^{-} \gamma \ldots \ldots \ldots$

4.2.3 Pseudoscalar form factors . . . . . . . .

4.2.4 $\eta$ decays into four charged particles . . .

$4.2 .5 \quad \eta \rightarrow \pi^{0} \gamma \gamma \ldots \ldots \ldots$

4.2.6 $\eta-\eta^{\prime}$ mixing and gluonium content . .

4.3 Low-Mass Scalars . . . . . . . . . . . . .

4.3.1 Structure of $f_{0}(980)$ and $a_{0}(980) \ldots$

4.3.2 Structure of the $\sigma$ meson . . . . . . . .

$4.3 .3 \phi \rightarrow K^{0} \bar{K}^{0} \gamma \ldots \ldots \ldots$

5 Physics in the Continuum: $\sigma_{\text {had }} \ldots \ldots \ldots$

5.1 SM precision tests and $\sigma_{\text {had }}$ at low energy . . .

5.2 The fine-structure constant at the $M_{Z}$ scale . .

5.3 The muon $g-2 \ldots \ldots \ldots$. . . . . . .

$5.4 \sigma_{\text {had }}$ measurements at low energy . . . . . . . .
5.5 Vector-meson spectroscopy . . . . . . . . . 36

5.6 VEPP-2000 prospects . . . . . . . . . . 37

5.7 Improving $\sigma_{\pi \pi}$ below $1 \mathrm{GeV}$ with KLOE-2 . . 38

5.8 Improving $\sigma_{\text {had }}$ above $1.02 \mathrm{GeV}$ with KLOE-2 38

5.9 Summary on $\sigma_{\text {had }}$ measurements . . . . . . . . 39

6 Physics in the Continuum: $\gamma \gamma$ Processes . . . . . . . 40

6.1 Scalar resonances in two photon collisions ... 40

$6.1 .1 e^{+} e^{-} \rightarrow e^{+} e^{-} \pi \pi$ at KLOE-2 . . . . . 42

6.2 Single pseudoscalar final states . . . . . . . . 44

6.2.1 Two photon width of pseudoscalar mesons 44

6.2.2 Contribution to Light-by-Light scattering 46

7 Hidden WIMP Dark Matter . . . . . . . . . . . . . 47

8 Summary ...................... 51

\section{Introduction}

This report results from discussion started at the workshop held at the Frascati Laboratory of INFN to review the major topics of interest for investigation at the upgraded $\phi$-factory DA $\Phi$ NE . The scientific program with a high-performance detector such as KLOE covers several fields in particle physics: from measurements of interest for the development of the Effective Field Theory (EFT) in quark-confinement regime to fundamental tests of Quantum Mechanics (QM) and CPT invariance. It includes precision measurements to probe lepton universality, CKM unitarity and settle the hadronic vacuum polarization contribution to the anomalous magnetic moment of the muon and to the fine-structure constant at the $M_{Z}$ scale.

During year 2008 the Accelerator Division of the Frascati Laboratory has tested a new interaction scheme on the DA $\Phi N E$-factory collider, with the goal of reaching a peak luminosity of $5 \times 10^{32} \mathrm{~cm}^{-2} \mathrm{~s}^{-1}$, a factor of three larger than what previously obtained. The test has been successful and presently DA $\Phi$ NE is delivering up to 15 $\mathrm{pb}^{-1} /$ day, with solid hopes to reach soon $20 \mathrm{pb}^{-1} /$ day [1,2]. Following these achievements, the data-taking campaign of the KLOE detector on the improved machine that was proposed in 2006 [3, will start in 2010.

KLOE is a multipurpose detector, mainly consisting of a large cylindrical drift chamber with an internal radius of $25 \mathrm{~cm}$ and an external one of $2 \mathrm{~m}$, surrounded 
by a lead-scintillating fibers electromagnetic calorimeter. Both are immersed in the 0.52 $\mathrm{T}$ field of a superconducting solenoid. Peculiar to KLOE is the spherical, $10 \mathrm{~cm}$ radius, beam pipe which allows all of the $K_{S}^{0}$ mesons produced in $\phi$ decays to move in vacuum before decaying. Details of the detector can be found in Refs. 4,5,6,7,8. From 2000 to 2006 , KLOE has acquired $2.5 \mathrm{fb}^{-1}$ of data at the $\phi(1020)$ peak, plus additional $250 \mathrm{pb}^{-1}$ at energies slightly higher or lower than that. The $\phi$ meson predominantly decays into charged and neutral kaons, thus allowing KLOE to make precision studies in the fields of flavor physics and low energy QCD. The latter can also be addressed using $\phi$ radiative decays into scalar or pseudoscalar particles. Test of discrete symmetries conservation can be performed using several different methods. Most notably, CPT conservation can be tested via quantum interferometry measurements with neutral kaons, a technique which exploits the quantum correlation between the $K_{L}^{0}$ and the $K_{S}^{0}$ produced in $\phi$ decays. A collection of the main physics results of KLOE can be found in Ref. 9.

For the forthcoming run [10, upgrades have also been proposed for the detector. In a first phase, two different devices (LET and HET) will be installed along the beam line to detect the scattered electrons/positrons from $\gamma \gamma$ interactions. In a second phase, a light-material internal tracker (IT) will be installed in the region between the beam pipe and the drift chamber to improve charged vertex reconstruction and to increase the acceptance for low $\mathrm{p}_{T}$ tracks 11. Crystal calorimeters (CCALT) will cover the low $\theta$ region, aiming at increasing acceptance for very forward electrons/photons down to $8^{\circ}$. A new tile calorimeter (QCALT) will be used to instrument the DA $\Phi$ NE focusing system for the detection of photons coming from $K_{L}$ decays in the drift chamber. Implementation of the second phase is planned for late 2011. The integrated luminosity for the two phases, from here on dubbed as step- 0 and step- 1 , will be $5 \mathrm{fb}^{-1}$ and $20 \mathrm{fb}^{-1}$, respectively.

$\mathrm{DA} \Phi \mathrm{NE}$ can run in a range of $\pm 20 \mathrm{MeV}$ from the $\phi$ peak without loss of luminosity, with the same magnetic configuration. Minor modifications, i.e., a new final particle focusing system, are needed to extend the range to $\pm 100 \mathrm{MeV}$ while a major upgrade of the machine is required to extend it above this limit. The improved KLOE detector is perfectly suited for taking data also at energies away from the $\phi$ mass. Therefore a proposal to perform the challenging and needed precision measurements of (multi)hadronic and $\gamma \gamma$ cross sections at energies up to $2.5 \mathrm{GeV}$ has also been put forward.

We will refer to the entire plan of run as the KLOE-2 project.

The present paper is organised into sections, devoted to the main physics topics of the experiment, where we briefly review the present-day situation for the theoretical and experimental achievements and the improvements expected for the next few years, paying particular attention to the discussion of the KLOE-2 contribution in the field.
We begin in Sect. 2 with precision measurements of CKM unitarity and lepton universality. Section 3 is devoted to the tests of QM and CPT invariance and particurarly to the study of the entangled kaon states. Issues on lowenergy QCD of interest for Chiral Perturbation Theory (ChPT) and for the modelization of underlying quark dynamics are addressed in Sect. 4, while the two following sections, Sects. 56 discuss the physics in the continuum, with the measurements of (multi)hadronic cross sections and $\gamma \gamma$ processes. Section 7 presents the program for searches in the hidden particle sector proposed by recent papers on the Dark Matter (DM) quest. Finally, in Sect. 8 we summarize our proposal of physics reach at the upgraded DA $\Phi$ NE .

\section{CKM Unitarity and Lepton Universality}

Purely leptonic and semileptonic decays of $\mathrm{K}$ mesons $(K \rightarrow$ $\ell \nu, K \rightarrow \pi \ell \nu, \ell=e, \mu)$ are mediated in the Standard Model (SM) by tree-level W-boson exchange. Gauge coupling universality and three-generation quark mixing imply that semileptonic processes such as $d^{i} \rightarrow u^{j} \ell \nu$ are governed by the effective Fermi constant $G_{i j}=G_{\mu} V_{i j}$, where: (i) $G_{\mu}$ is the muon decay constant; (ii) $V_{i j}$ are the elements of the unitary Cabibbo-Kobayashi Maskawa (CKM) matrix. This fact has simple but deep consequences, that go under the name of universality relations:

- In the SM the effective semileptonic constant $G_{i j}$ does not depend on the lepton flavor (lepton universality).

- If one extracts $V_{i j}$ from different semileptonic transitions assuming quark-lepton gauge universality (i.e. normalizing the decay rates with $G_{\mu}$ ), the CKM unitarity condition $\sum_{j}\left|V_{i j}\right|^{2}=1$ should be verified.

Beyond the SM, these universality relations can be violated by new contributions to the low-energy $V-A$ four fermion operators, as well as new non $V$-A structures. Therefore, precision tests of the universality relations probe physics beyond the SM and are sensitive to several SM extensions 12, 13, 14, 15. A simple dimensional analysis argument reveals that deviations from the universality relations scale as $\Delta \sim M_{W}^{2} / \Lambda^{2}$, where $\Lambda$ is the scale associated with new physics (NP). Therefore, testing the universality relations at the $0.1 \%$ level allows us to put non-trivial constraints on physics scenarios at the $\mathrm{TeV}$ scale that will be directly probed at the LHC. Kaon physics currently plays a prominent role in testing both quark-lepton universality, through the $V_{u s}$ entry in the CKM unitarity relation $\left|V_{u d}\right|^{2}+\left|V_{u s}\right|^{2}+\left|V_{u b}\right|^{2}=1$, and lepton universality, through the measurement of $\left|V_{u s}\right| \times f_{+}(0)$ and the helicity suppressed ratio $\Gamma(K \rightarrow e \nu(\gamma)) / \Gamma(K \rightarrow \mu \nu(\gamma))$. In the case of the latter, theoretical prediction, at the 0.04 $\%$ level, is extremely clean [16,17] (it is only affected by hadronic structure dependence to two loops in the chiral effective theory).

The experimental precision was at the $6 \%$ level 18 before the recently-published KLOE measurement [19] which has improved the sensitivity to $1 \%$. New results from the NA62 collaboration at CERN are expected to reduce in 
near-term the error to the $0.5 \%$ level. This helicity suppressed ratio is very promising in terms of uncovering physics beyond the SM [20], including tests of Higgs-induced lepton flavor violation in supersymmetry 21 . Improvements on the experimental side are highly motivated.

Large amount of data has been collected on the semileptonic modes $K \rightarrow \pi \ell \nu$ by several experiments, BNL-E865, KLOE, KTeV, ISTRA+, and NA48 in the last few years. These data have stimulated a substantial progress on the theoretical inputs, so that most of the theory-dominated errors associated to radiative corrections $22,23,24,25$ and hadronic form factors 20 have been reduced below $1 \%$. Presently, the unitarity test

$$
\left|V_{u d}\right|^{2}+\left|V_{u s}\right|^{2}+\left|V_{u b}\right|^{2}=1+\Delta_{\mathrm{CKM}}
$$

implies that $\Delta_{\mathrm{CKM}}$ is consistent with zero at the level of $6 \times 10^{-4}$. $V_{u s}$ from $K \rightarrow \pi \ell \nu$ decays contributes about half of this uncertainty, mostly coming from the hadronic matrix element. Both experimental and theoretical progress in $K_{\ell 3}$ decays will be needed in order to improve the accuracy on $\Delta_{\mathrm{CKM}}$ in the future.

The kaon semileptonic decay rate is given by:

$$
\begin{gathered}
\Gamma\left(K_{l 3}\right)=\frac{C_{K}^{2} G_{F}^{2} M_{K}^{5}}{192 \pi^{3}} S_{E W}\left|V_{u s}\right|^{2}\left|f_{+}(0)\right|^{2} \times \\
I_{K, l}(\lambda)\left(1+2 \Delta_{K}^{S U(2)}+2 \Delta_{K, l}^{E M}\right)
\end{gathered}
$$

where $K=K^{0}, K^{ \pm}, l=e, \mu$ and $C_{K}$ is a Clebsch-Gordan coefficient, equal to $1 / 2$ and 1 for $K^{ \pm}$and $K^{0}$, respectively. The decay width $\Gamma\left(K_{l 3}\right)$ is experimentally determined by measuring the kaon lifetime and the semileptonic branching fractions (BRs) totally inclusive of radiation. The theoretical inputs are: the universal short-distance electroweak correction $S_{E W}=1.0232$, the $S U(2)$-breaking $\Delta_{K}^{S U(2)}$ and the long-distance electromagnetic corrections $\Delta_{K, l}^{E M}$ which depend on the kaon charge and on the lepton flavor, and the form factor $f_{+}(0) \equiv f_{+}^{K^{0} \pi^{-}}(0)$ parametrizing the hadronic matrix element of the $K \rightarrow \pi$ transition, evaluated at zero momentum transfer and for neutral kaons. The form factor dependence on the momentum transfer is needed for the calculation of the phase space integral $I_{K, l}(\lambda)$. It can be described by one or more slope parameters $\lambda$ measured from the decay spectra.

Complementary to $K_{\ell 3}$ decays, the kaon (pion) leptonic radiation-inclusive decays $K(\pi) \rightarrow \mu \bar{\nu}_{\mu}(\gamma)$ provide a precise determination of $\left|V_{u s}\right| /\left|V_{u d}\right|$. The ratio of these decay rates is:

$$
\frac{\Gamma\left(K_{\mu 2}^{+}\right)}{\Gamma\left(\pi_{\mu 2}^{+}\right)}=\frac{m_{K}\left(1-\frac{m_{\mu}^{2}}{m_{K}^{2}}\right)^{2}}{m_{\pi}\left(1-\frac{m_{\mu}^{2}}{m_{\pi}^{2}}\right)^{2}} \frac{f_{K}^{2}\left|V_{u s}\right|^{2}}{f_{\pi}^{2}\left|V_{u d}\right|^{2}} \frac{1+\frac{\alpha}{\pi} C_{K}}{1+\frac{\alpha}{\pi} C_{\pi}}
$$

where $f_{K}$ and $f_{\pi}$ are, respectively, the kaon and the pion decay constants; $C_{\pi}$ and $C_{K}$ parametrize the radiationinclusive electroweak corrections accounting for bremsstrahlung emission of real photons and virtual-photon loop contributions.
It has been shown that presently semileptonic processes and the related universality tests provide constraints on NP that cannot be obtained from other electroweak precision tests and/or direct measurements at the colliders 15.

In the following subsections, an overview of the present scenario and possible improvements on results from kaon physics will be given on both theoretical and experimental sides.

\subsection{Determination of $f_{+}(0)$ and $f_{K} / f_{\pi}$}

The vector form factor at zero-momentum transfer, $f_{+}(0)$, is the key hadronic quantity required for the extraction of the CKM matrix element $\left|V_{u s}\right|$ from semileptonic $K_{\ell 3}$ decays as in Eq.(2). Within $\mathrm{SU}(3)$ ChPT one can perform a systematic expansion of $f_{+}(0)$ of the type

$$
f_{+}(0)=1+f_{2}+f_{4}+\ldots,
$$

where $f_{n}=\mathcal{O}\left[M_{K, \pi}^{n} /\left(4 \pi f_{\pi}\right)^{n}\right]$ and the first term is equal to one due to the vector current conservation in the $\mathrm{SU}(3)$ limit. Because of the Ademollo-Gatto (AG) theorem [26], the first non-trivial term $f_{2}$ does not receive contributions from the local operators of the effective theory and can be unambiguously computed in terms of the kaon and pion masses $\left(m_{K}\right.$ and $\left.m_{\pi}\right)$ and the pion decay constant $f_{\pi}$. At the physical point the value is $f_{2}=-0.023$ [27. Thus the problem is reduced to the evaluation of $\Delta f$, defined as

$$
\Delta f \equiv f_{4}+f_{6}+\ldots=f_{+}(0)-\left(1+f_{2}\right),
$$

which depends on the low-energy constants (LECs) of the effective theory and cannot be deduced from other processes. The original estimate made by Leutwyler and Roos (LR) 27 was based on the quark model yielding $\Delta f=$ $-0.016(8)$. More recently other analytical approaches have been devised to obtain the next-to-next-to-leading (NNLO) order term $f_{4}$ by writing it as:

$$
f_{4}=L_{4}(\mu)+f_{4}^{l o c}(\mu),
$$

where $\mu$ is the renormalization scale, $L_{4}(\mu)$ is the loop contribution computed in Ref. 28 and $f_{4}^{l o c}(\mu)$ is the $\mathcal{O}\left(p^{6}\right)$ local contribution. For the latter, various models have been adopted obtaining values compatible with zero within uncertainty leading to $f_{+}(0)$ values significantly larger than the LR estimate (cf. Tab. 1) and to the prediction of smaller SU(3)-breaking effects. Even if in principle the NNLO term $f_{4}$ could be obtained from the measurement of the slope $\left(\lambda_{0}^{\prime}\right)$ and the curvature $\left(\lambda_{0}^{\prime \prime}\right)$ of the scalar form factor $f_{0}\left(q^{2}\right)$, this is experimentally impossible. Even in the case of a perfect knowledge of $\lambda_{+}^{\prime}$ and $\lambda_{+}^{\prime \prime}$, with one million of $K_{\mu 3}$ events, the error $\delta \lambda_{0}^{\prime \prime}$ would be about four times the expected value of $\lambda_{0}^{\prime \prime}$. Thus, one has to rely on dispersion relations which allow the calculation of the curvature from the measurement of only one parameter and then to perform a matching with ChPT [29]. At present, the precision reached is not sufficient for an accurate determination of $f_{+}(0)$. 
A precise evaluation of $f_{+}(0)$, or equivalently $\Delta f$, requires the use of non-perturbative methods based on the fundamental theory of the strong interaction, such as lattice QCD simulations. Such precision determinations began recently with the quenched simulations of Ref. 30, where it was shown that $f_{+}(0)$ can be determined at the physical point with $\simeq 1 \%$ accuracy. The findings of Ref. [30 triggered various unquenched calculations of $f_{+}(0)$, with both $N_{f}=2$ and $N_{f}=2+1$ dynamical flavors. The error associated with the chiral extrapolation was significantly reduced thanks to the lighter pion masses ( the minimal value of the simulated pion mass is $260 \mathrm{MeV}$ ). The results for $f_{+}(0)$ are summarized in Tab. 1. It can be seen that all lattice results are in agreement with the LR estimate, while the analytical approaches of Refs. [28, 31, 32 are systematically higher. Since simulations of lattice

Table 1: Summary of model and lattice results for $f_{+}(0)$. The lattice errors include both statistical and systematic uncertainties, unless otherwise indicated.

\begin{tabular}{|c|c|c|}
\hline Ref. & Model/Lattice & $f_{+}(0)$ \\
\hline 27 & $\overline{\overline{L R}}$ & $0.961(8)$ \\
\hline 28 & $C h P T+L R$ & $0.978(10)$ \\
\hline 31 & ChPT+disp. & $0.974(11)$ \\
\hline 32 & $C h P T+1 / N_{c}$ & $0.984(12)$ \\
\hline 33 & $C h P T+1 / N_{c}$ & $0.986(7)$ \\
\hline 30 & $\overline{\overline{S P Q_{c d} R}}$ & $0.960(9)$ \\
\hline 34 & $\overline{J J L Q C D}$ & $0.967(6)$ \\
\hline 35 & $R B C$ & $0.968(12)$ \\
\hline 36 & $Q C D S F$ & $0.965\left(2_{\text {stat }}\right)$ \\
\hline 37 & $E T M C$ & $0.957(8)$ \\
\hline 38 & 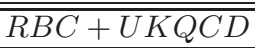 & $0.964(5)$ \\
\hline
\end{tabular}

QCD are carried out in a finite volume, the momentum transfer $q^{2}$ for the conventionally used periodic fermion boundary conditions takes values corresponding to the Fourier modes of the kaon or pion. Using a phenomenological ansatz for the $q^{2}$-dependence of the form factor one interpolates to $q^{2}=0$ where $f_{+}(0)$ is extracted, thereby introducing a major systematic uncertainty. A new method based on the use of partially twisted boundary conditions has been developed 39 which allows this uncertainty to be entirely removed by simulating directly at the desired kinematical point $q^{2}=0$. A systematic study of the scaling behavior of $f_{+}(0)$, using partially twisted boundary conditions and the extension of the simulations to lighter pion masses in order to improve the chiral extrapolation are priorities for the upcoming lattice studies of $K_{\ell 3}$ decays.

In contrast to $f_{+}(0)$, the pseudoscalar decay constants $f_{K}$ and $f_{\pi}$ are not protected by the AG theorem [26] against linear corrections in the $\mathrm{SU}(3)$ breaking. Moreover the first non-trivial term in the chiral expansion of $f_{K} / f_{\pi}$, of order $\mathcal{O}\left(p^{4}\right)$, depends on the LECs and therefore it cannot be unambiguously predicted within ChPT.
This is the reason why the most precise determinations of $f_{K} / f_{\pi}$ come from lattice QCD simulations.

In recent years various collaborations have provided new results for $f_{K} / f_{\pi}$ using unquenched gauge configurations with both $N_{f}=2$ and $N_{f}=2+1$ dynamical flavors. They are summarized in Tab. 2. At the current level of precision, the comparison of $N_{f}=2$ and $N_{f}=2+1$ results indicate a rather small contribution of the strange sea quarks to the ratio of the decay constants. Table 2

Table 2: Summary of lattice results for $f_{K} / f_{\pi}$. The errors include both statistical and systematic uncertainties.

\begin{tabular}{|c|c|c|}
\hline Ref. & Collaboration & $f_{K} / f_{\pi}$ \\
\hline \begin{tabular}{|l|l}
40,41 \\
\end{tabular} & $\overline{M I L C}$ & $1.197_{-13}^{+7}$ \\
\hline 42 & $\overline{H P Q C D}$ & $1.189(7)$ \\
\hline 43 & $\overline{B M W}$ & $1.192(9)$ \\
\hline 44 & $\overline{E T M C}$ & $1.210(18)$ \\
\hline 45 & Aubin et al. & $1.191(23)$ \\
\hline 466 & $\overline{N P L Q C D}$ & $1.218_{-24}^{+11}$ \\
\hline 47 & $R B C / U K Q C D$ & $1.205(65)$ \\
\hline 48 & $P A C S-C S$ & $1.189(20)$ \\
\hline
\end{tabular}

deserves few comments: i) the convergence of the $\mathrm{SU}(3)$ chiral expansion for $f_{K} / f_{\pi}$ is quite questionable, mainly because large next-to-leading order (NLO) corrections are already required to account for the large difference between the experimental value of $f_{\pi}$ and the value of this decay constant in the massless $\mathrm{SU}(3)$ limit. Instead, the convergence of the $\mathrm{SU}(2)$ chiral expansion is much better and, thanks to the light pion masses reached in recent lattice calculations, the uncertainty related to chiral extrapolation to the physical point is kept at the percent level [47; ii) little is known about the details of the chiral and continuum extrapolation in Ref. 42 (HPQCD) which is currently the most precise lattice prediction for $f_{K} / f_{\pi}$; iii) there is also some concern about the staggered fermion formulation used by MILC 40,41, HPQCD 42, Aubin et al. 45 and NPLQCD 46. These results would have to be confirmed by conceptually clean fermion formulations.

Therefore it is not obvious to decide which is the value to be used for $f_{+}(0)$ and $f_{K} / f_{\pi}$. A dedicated working group, the FLAVIAnet lattice averaging group (FLAG), has just started to compile and publish lattice QCD results for SM observables and parameters. They have identified those published results demonstrating good control over systematic uncertainties. A first status report is given in Ref. 49.

\subsection{Experimental results from kaon decays}

In the last years, many efforts have been dedicated to the correct averaging of the rich harvest of recent results in kaon physics. The FLAVIAnet kaon working group has published a comprehensive review [20] where a detailed description of the averaging procedure can be found. In 
this paper we will focus on the contribution from KLOE, with both the present data set $\left(2.5 \mathrm{fb}^{-1}\right)$ and the $5 \mathrm{fb}^{-1}$ planned for KLOE-2/step-0 (cf. Sect. 1).

After four years of data analysis, KLOE has produced the most comprehensive set of results from a single experiment, measuring the main BRs of $K_{L}$ [50, $K^{ \pm}$[51,52,53. and $K_{S}$ [54,55] (unique to KLOE), including semileptonic and two-body decays; lifetime measurements for $K_{L} \square 5$ and $K^{ \pm}$[57]; form factor slopes from the analysis of $K_{L} e 3$ [58 and $K_{L} \mu 3$ [59,60. The value of $\left|V_{u s}\right| \times f_{+}(0)$ has been obtained 61] using the $K_{S}$ lifetime from PDG [18 as the only non-KLOE input. These data together with the value of $\left|V_{u s}\right| /\left|V_{u d}\right|$ from the measurement of the $K^{ \pm} \rightarrow \mu^{ \pm} \nu(\gamma)$ branching ratio 52 and the extraction of $\left|V_{u d}\right|$ from superallowed nuclear $\beta$ decays, provide the basis for testing the unitarity of the quark-flavor mixing matrix. A test of lepton universality and improvements of the bounds on $\mathrm{NP}$ scenarios have also obtained from the measurements of $\left|V_{u s}\right|$ from leptonic and semileptonic decays.

\subsubsection{Branching ratios}

KLOE has measured all of the main branching ratios of $K_{L}, K_{S}$, and $K^{ \pm}$decays using for the $K_{L}$ the whole data set and only one fifth of the entire sample for $K_{S}$ and $K^{ \pm}$. A summary of the results on $K_{L}, K_{S}$, and $K^{ \pm}$is shown in Tab. 3. The measurement of the absolute BR of the $K^{ \pm}$semileptonic decays on the basis of the entire data sample of $2.5 \mathrm{fb}^{-1}$ has just started. The analysis of the subdominant $K_{S} e 3$ modes will be improved at KLOE-2 also with the upgrade of the tracking system through the installation of the inner chamber (cf. Sect. 1). Preliminary studies have shown that the upgrade will increase i) the acceptance for low-momentum tracks and ii) the vertex resolution for decays close to the beam interaction point by a factor of three.

Table 3: Summary of KLOE results on $K_{L}, K_{S}$, and $K^{ \pm}$ branching ratios.

\begin{tabular}{|c|c|}
\hline$K_{L} \rightarrow \pi e \nu$ & $0.4008 \pm 0.0015[50$ \\
\hline$K_{L} \rightarrow \pi \mu \nu$ & $0.2699 \pm 0.0014[50$ \\
\hline$K_{L} \rightarrow 3 \pi^{0}$ & $0.1996 \pm 0.002050$ \\
\hline$K_{L} \rightarrow \pi^{+} \pi^{-} \pi^{0}$ & $0.1261 \pm 0.0011[\underline{50}$ \\
\hline$K_{L} \rightarrow \pi^{+} \pi^{-}$ & $(1.963 \pm 0.21) \times 10^{-3} 62$ \\
\hline$K_{L} \rightarrow \gamma \gamma$ & $(5.569 \pm 0.077) \times 10^{-4} 63$ \\
\hline$K_{S} \rightarrow \pi^{+} \pi^{-}$ & $0.60196 \pm 0.0005155$ \\
\hline$K_{S} \rightarrow \pi^{0} \pi^{0}$ & $0.30687 \pm 0.0005155$ \\
\hline$K_{S} \rightarrow \pi e \nu$ & $(7.05 \pm 0.09) \times 10^{-4}[54$ \\
\hline$K_{S} \rightarrow \gamma \gamma$ & $(2.26 \pm 0.13) \times 10^{-6}$ \\
\hline$K_{S} \rightarrow 3 \pi^{0}$ & $<1.2 \times 10^{-7}$ at $90 \%$ C.L. 65 \\
\hline$K_{S} \rightarrow e^{+} e^{-}(\gamma)$ & $<9 \times 10^{-9}$ at $90 \%$ C.L. 66 \\
\hline$K^{+} \rightarrow \mu^{+} \nu(\gamma)$ & $0.6366 \pm 0.0017[52$ \\
\hline$K^{+} \rightarrow \pi^{+} \pi^{0}(\gamma)$ & $0.2067 \pm 0.001253$ \\
\hline$K^{+} \rightarrow \pi^{0} e^{+} \nu(\gamma)$ & $0.04972 \pm 0.0005351$ \\
\hline$K^{+} \rightarrow \pi^{0} \mu^{+} \nu(\gamma)$ & $0.03237 \pm 0.0003951$ \\
\hline$K^{+} \rightarrow \pi^{+} \pi^{0} \pi^{0}$ & $0.01763 \pm 0.0003467$ \\
\hline
\end{tabular}

\subsubsection{Kaon lifetimes}

KLOE has measured the $K_{L}$ [56, 50 and the $K^{ \pm}$lifetimes 57 using approximately one fifth of the collected data set. Two independent measurements of the $K_{L}$ lifetime have been obtained, yielding an overall fractional accuracy of $1 \%$ :

$$
\begin{aligned}
& \text { (a) } \tau_{L}=(50.92 \pm 0.17 \pm 0.25) \mathrm{ns} \\
& \text { (b) } \tau_{L}=(50.72 \pm 0.11 \pm 0.35) \mathrm{ns}
\end{aligned}
$$

The first measurement uses the fit to the proper-time distribution of the $K_{L} \rightarrow \pi^{0} \pi^{0} \pi^{0}$ decay channel and is currently being repeated with the complete data set. From the preliminary result, $\tau_{L}=(50.56 \pm 0.14 \pm 0.21) \mathrm{ns}$ 68, a fractional error of $0.38 \%$ is expected on the final measurement. Adding the $5 \mathrm{fb}^{-1}$ from the first year of datataking with KLOE-2, the fractional accuracy is reduced to $0.27 \%$. Furtermore, the insertion of new quadrupole instrumentation [69] as planned in 2011 will improve the photon reconstruction allowing better control of the systematics and leading to an error $\Delta \tau_{L} / \tau_{L}<0.2 \%$.

The second $\tau_{L}$ measurement was obtained from the simultaneous measurements of the main $K_{L}$ branching ratios, imposing the constraint on the their sum, $\sum B R_{i}=$ 1. This technique yielded a result of remarkable precision, but severely dominated by systematics.

With the available sample at KLOE-2 and the improved tracking performance from the installation of the IT, it is possible to exploit the measurement of the propertime distribution of all of the charged modes to further improve on $\tau_{L}$. Critical point of this approach is the control of small variations in the tag efficiency of different decay channels. Succeeding in the latter, the result combined with the measurement obtained from the neutral channel would reduce the relative error on $\tau_{L}$ to $0.1 \%$.

The $K^{ \pm}$lifetime has been determined by KLOE with two independent tecnhiques [57: the measurement of the decay length by reconstructing the $K^{ \pm}$track, and of the decay time with a time-of-flight technique using modes with one $\pi^{0}$ in the final state $\left(K^{ \pm} \rightarrow \pi^{0} X\right)$. Their combination yields a relative error of $0.25 \%$.

$$
\begin{aligned}
& \text { decay length } \tau_{ \pm}=(12.364 \pm 0.031 \pm 0.031) \mathrm{ns} \\
& \text { decay time } \tau_{ \pm}=(12.337 \pm 0.030 \pm 0.020) \mathrm{ns}
\end{aligned}
$$

For both methods there is still room for improving both statistical and systematic errors by increasing the analyzed sample. Making use of the KLOE and $5 \mathrm{fb}^{-1}$ of the KLOE-2 data sample, the $\tau_{ \pm}$relative error can be reduced to the $0.1 \%$ level. Moreover, the decay length technique would become much more accurate with the inner tracker allowing the detection of $K^{ \pm}$tracks closer to the interaction point. This is the step- 1 scenario, aiming to improve by a factor of two $\Delta \tau_{ \pm} / \tau_{ \pm}$.

Finally, a new promising technique is currently being developed at KLOE to measure the $K_{S}$ lifetime. This consists in an event-by-event determination of the $\phi$ and the $K_{S}$ decay-point positions by a kinematic fit. With the full KLOE statistics the preliminary result $\tau_{S}=(89.56 \pm$ $0.03 \pm 0.07)$ ps 68 , has been obtained, with the aim of reaching $\sim 0.03$ ps final systematic uncertainty. A relative 
error of $0.03 \%$ on $\tau_{S}$ is expected scaling this result to the KLOE-2/step-0 data sample.

\subsubsection{Kaon form factors}

To compute the phase space integrals appearing in the SM photon-inclusive $K_{\ell 3}$ decay rates as in Eq. (2), we need experimental or theoretical inputs about the $t$-dependence of $f_{+, 0}(t)$. In principle, ChPT and Lattice QCD are useful to set theoretical constraints. In practice, at present the $t$ dependence of the form factors (FFs) is better determined by measurements and by combining measurements with dispersion relations.

A complete description of the experimental situation on FFs can be found in Ref. 20. KLOE-2 can contribute to improve present knowledge of FF. Besides, precise measurements of the FF properties impose stringent test on quantities which can be obtained only from theory. In particular, the theoretical determination of $f_{+}(0)$ (from Lattice and ChPT), and the ratio of the kaon and pion decay constants defined in the $m_{u}=m_{d}$ and $\alpha_{\mathrm{em}} \rightarrow 0$ limit, $f_{K} / f_{\pi}$ (from Lattice only).

In the physical region, $m_{\ell}^{2}<t<\left(m_{K}-m_{\pi}\right)^{2}$, a very good approximation of the FFs is given by a Taylor expansion up to $t^{2}$ terms

$\tilde{f}_{+, 0}(t) \equiv \frac{f_{+, 0}(t)}{f_{+}(0)}=1+\lambda_{+, 0}^{\prime} \frac{t}{m_{\pi}^{2}}+\frac{1}{2} \lambda_{+, 0}^{\prime \prime}\left(\frac{t}{m_{\pi}^{2}}\right)^{2}+\ldots$

For the vector form factor $\tilde{f}_{+}$, the experimental information from both $K_{e 3}$ and $K_{\mu 3}$ data is quite accurate and so far superior to theoretical predictions. For the scalar form factor, $\tilde{f}_{0}(t)$, the situation is more complicated: i) first of all $\tilde{f}_{0}(t)$ is accessible from $K_{\mu 3}$ data only; ii) the correlation between $\lambda_{+, 0}^{\prime}$ and $\lambda_{+, 0}^{\prime \prime}$ is close to -1 so that a fit will trade $\lambda_{+, 0}^{\prime}$ for $\lambda_{+, 0}^{\prime \prime}$ and consequently enlarge the errors, on the other hand, ignoring the $t^{2}$ term would increase the value of $\lambda_{+, 0}^{\prime}$ by $\sim 3.5 \lambda_{+, 0}^{\prime \prime}$; iii) it is not fully justified to fit with two parameters related by the simple relation $\lambda_{+, 0}^{\prime \prime}=2 \lambda_{+, 0}^{\prime}{ }^{2}$ from the pole parametrisation. Indeed, if for the vector $\mathrm{FF}$ the $\mathrm{K}^{\star}(892)$ resonance dominates, for the scalar FF there is not such an obvious pole dominance. Instead, a suitable single-parameter function could be introduced for $\tilde{f}_{+, 0}(t)$ using a dispersive approach as emphasized in Refs. 20,70,71. A dispersive parametrization for $\tilde{f}_{+}$has been built in Ref. [71, with good analytical and unitarity properties and a correct threshold behaviour. For the scalar FF, particularly appealing is an improved dispersion relation proposed in Ref. [71,72 where two subtractions are performed, at $t=0$ (where by definition, $\left.\tilde{f}_{0}(0) \equiv 1\right)$, and at the so-called Callan-Treiman point $t_{C T} \equiv\left(m_{K}^{2}-m_{\pi}^{2}\right)$. At this second point, the CallanTreiman theorem 73.74 implies

$$
\tilde{f}_{0}\left(t_{C T}\right)=\frac{f_{K}}{f_{\pi}} \frac{1}{f_{+}(0)}+\Delta_{C T}
$$

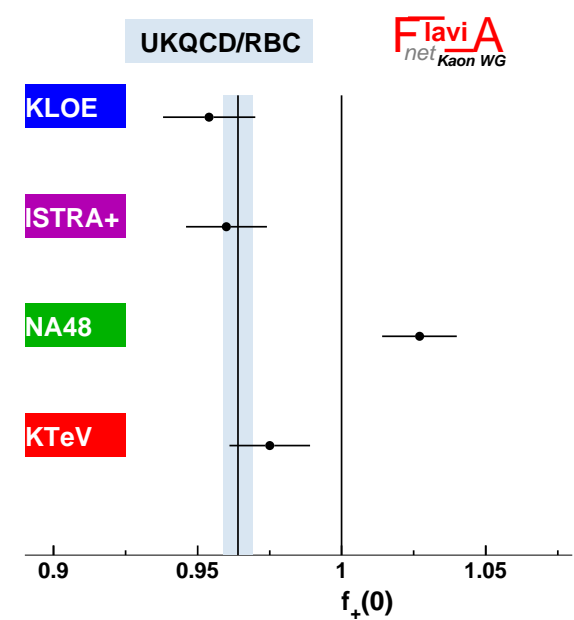

Fig. 1: Values of $f_{+}(0)$ determined from experimental measurement of the scalar FF slope compared with the lattice calculations from the UKQCD/RBC collaboration.

where $\Delta_{C T} \sim \mathcal{O}\left(m_{u, d} / 4 \pi F_{\pi}\right)$ is a small quantity which can be estimated from theory ( ChPT at NLO in the isospin limit estimates $\left.\Delta_{C T}=(-3.5 \pm 8) \times 10^{-3}\right)[75$.

Using $\sim 500 \mathrm{pb}^{-1}$ of integrated luminosity KLOE has obtained the vector $\mathrm{FF}$ in $K_{L} e 3$ decays [58] and the vector and scalar $\mathrm{FF}$ in $K_{L} \mu 3$ decays [59]. For scalar and vector FFs measured in $K_{L} \mu 3$ events, KLOE has also presented the preliminary result based on an integrated luminosity of about $1.5 \mathrm{fb}^{-1} 60$. The statistical error on FF should improve by a factor of three with respect to the KLOE published measurements with the analysis of KLOE-2/step-0 data. Since in KLOE the systematic errors are partially statistical in nature (efficiencies are measured with selected control samples and downscaled samples are commonly used) also these contributions to the total uncertainty decrease approximately by a comparable amount.

For $K^{ \pm}$semileptonic FF the situation is different: the analyses of the KLOE data sample has just started. The main difference with respect to the $K_{L}$ analysis is the absence of the ambiguity on the lepton charge assignment due to the unique charge of the $K^{ \pm}$decay product. Moreover, due to the $\pi^{0}$ presence, for $K^{ \pm} \mu 3$ there is not the $\pi-\mu$ ambiguity which is a limiting systematic factor on the $K_{L} \mu 3 \mathrm{FF}$ measurement. All new FF measurements will use dispersive approaches to fit the data.

The Callan-Treiman relation fixes the value of scalar form factor at $t_{C T}$ to be equal to the $\left(f_{K} / f_{\pi}\right) / f_{+}(0)$ ratio as in Eq. (10). The dispersive parametrization for the scalar form factor proposed in Ref. [72] allows a precise determination of $f_{K} / f_{\pi} / f_{+}(0)$ from the available measurements of the scalar form factor. This experimental evaluation is completely independent from lattice estimates. Figure 1 shows the experimental results on $f_{+}(0)$ from the scalar FF dispersive measurements, using Callan-Treiman relation and $f_{K} / f_{\pi}=1.189(7)$. The value of $f_{+}(0)=$ 
0.964(5) from lattice UKQCD/RBC is also shown for comparison. The NA48 result is difficult to accommodate and it violates the theoretical bound $f_{+}(0)<1$ pointing to the presence of right-handed currents from physics beyond the SM. The fit probability of the world $\tilde{f}_{0}(t)$ data (excluding NA48) is $39 \%$ and the average is in good agreement with lattice calculations. Improvements on the experimental determination of the ratio $f_{K} / f_{\pi} / f_{+}(0)$ can be very effective in constraining right-handed currents 72 and also for the validation of more precise lattice calculations.

\subsubsection{Kaon form factors from $\tau$ decays}

To complete the discussion on the determination of kaon semileptonic FFs we present the status of the analysis of $\tau \rightarrow K \pi \nu_{\tau}$ decays. The recent precise results by BaBar [76] and Belle 77] provide complementary, higher-energy measurements of the kaon form factors. Indeed, the differential decay distribution,

$$
\begin{gathered}
\frac{\mathrm{d} \Gamma_{K \pi}}{\mathrm{d} \sqrt{s}}=\frac{G_{F}^{2}\left|V_{u s}\right|^{2} m_{\tau}^{3}}{32 \pi^{3} s} S_{\mathrm{EW}}\left(1-\frac{s}{m_{\tau}^{2}}\right)^{2} \times \\
{\left[\left(1+2 \frac{s}{m_{\tau}^{2}}\right) q_{K \pi}^{3}\left|f_{+}(s)\right|^{2}+\frac{3 \Delta_{K \pi}^{2}}{4 s} q_{K \pi}\left|f_{0}(s)\right|^{2}\right],}
\end{gathered}
$$

is expressed in terms of $f_{+}(s)$, with $s=\left(p_{K}+p_{\pi}\right)^{2}$, the $K \pi$ vector form factor, and $f_{0}(s)$, the scalar FF. $S_{\mathrm{EW}}$ is an electroweak correction factor, $\Delta_{K \pi}=m_{K}^{2}-m_{\pi}^{2}$, and $q_{K \pi}$ is the absolute value of the momentum in the $K \pi$ center of mass reference frame,

$$
\begin{aligned}
q_{K \pi}(s)=\frac{1}{2 \sqrt{s}} & \sqrt{\left(s-\left(m_{K}+m_{\pi}\right)^{2}\right)\left(s-\left(m_{K}-m_{\pi}\right)^{2}\right)} \\
& \times \theta\left(s-\left(m_{K}+m_{\pi}\right)^{2}\right) .
\end{aligned}
$$

A fit to the measured decay distribution is performed to determine the FFs. Several FF parametrizations have been proposed $78,79,80,81$. The form factors are obtained in the framework of chiral theory with resonances imposing additional constraints from dispersion relations 79, 80, 81, whereas in Ref. 78 a coupled channel analysis has been performed taking into account, through analyticity requirements, the experimental information on elastic and inelastic $K \pi$ scattering from the LASS collaboration 82 .

The parametrizations relying on dispersion relations guarantee that the form factors fulfil the analyticity and unitarity properties. In this framework, a vector FF description as model independent as possible through a threetimes-subtracted dispersive parametrization has been proposed in Ref. 81,

$$
\tilde{f}_{+}(s)=\exp \left[a_{1} \frac{s}{m_{\pi^{-}}^{2}}+a_{2}\left(\frac{s}{m_{\pi^{-}}^{2}}\right)^{2}+a_{3}(s)\right]
$$

with

$$
a_{1}=\lambda_{+}^{\prime}, \quad a_{2}=\frac{1}{2}\left(\lambda_{+}^{\prime \prime}-\lambda_{+}^{\prime 2}\right)
$$

and

$$
a_{3}(s)=\frac{s^{3}}{\pi} \int_{\left(m_{K}+m_{\pi}\right)^{2}}^{\infty} d s^{\prime} \frac{\delta_{1}^{K \pi}\left(s^{\prime}\right)}{\left(s^{\prime}\right)^{3}\left(s^{\prime}-s-i \epsilon\right)} .
$$

Slope and curvature of the form factor, $\lambda_{+}^{\prime}$ and $\lambda_{+}^{\prime \prime}$, are obtained from a fit. The phase, $\delta_{1}^{K \pi}$, considered elastic and therefore equal to the $P$-wave elastic $I=1 / 2 K \pi$ scattering phase shift, is determined by a model relying on the inclusion of two resonances, $\mathrm{K}^{*}(892)$ and $\mathrm{K}^{*}(1410)$ whose parameters are also fitted from data. Due to the presence of the $\mathrm{K}^{*}(892)$ resonance which dominates the decay distribution between 0.8 and $1.2 \mathrm{GeV}$, the vector FF dominates this decay except for the threshold region where the scalar form factor plays an important role. With the present accuracy the scalar FF cannot be determined from data and is thus taken as an input to the analysis. The values of $\lambda_{+}^{\prime}$ and $\lambda_{+}^{\prime \prime}$ in Tab. 4 have been obtained in Ref. [81 from a fit to the Belle data. These are compared with the results from others parametrizations [78. [79, 80] and the measurements from $K_{e 3}$ analyses [20, 83 . The different precision results on $\lambda_{+}^{\prime}$ are in good agree-

Table 4: Recent results on the slope $\lambda_{+}^{\prime}$ and the curvature $\lambda_{+}^{\prime \prime}$ of the vector form factor in units of $10^{-3}$ from the analysis of $\tau \rightarrow K \pi \nu_{\tau}$ [81,78, 79, 80, and $K_{e 3}$ decays [20, 83.

\begin{tabular}{ccc}
\hline \hline Ref. & $\lambda_{+}^{\prime}\left[10^{-3}\right]$ & $\lambda_{+}^{\prime \prime}\left[10^{-3}\right]$ \\
\hline 81 & $24.66 \pm 0.77$ & $1.199 \pm 0.020$ \\
\hline 78 & $26.05_{-0.58}^{+0.21}$ & $1.29_{-0.04}^{+0.01}$ \\
79,80 & $25.20 \pm 0.33$ & $1.285 \pm 0.031$ \\
\hline 20 & $25.2 \pm 0.9$ & $1.6 \pm 0.4$ \\
83 & $25.17 \pm 0.58$ & $1.22 \pm 0.10$ \\
\hline \hline
\end{tabular}

ment. The curvature $\lambda_{+}^{\prime \prime}$ from the average of recent $K_{e 3}$ measurements [20, using a quadratic Taylor parametrization has $25 \%$ uncertainty reduced to $8 \%$ by the dispersive parametrization of the FF 83 .

The knowledge of the t-dependence of vector FF and thus the $K_{e 3}$ phase space integral in Eq.(2) can be improved by combining $\tau$ and $K_{e 3}$ results. The potentiality of such a combined analysis has been presented in Ref. [84] leading to the following results:

$\lambda_{+}^{\prime}=(25.10 \pm 0.44) \times 10^{-3}, \lambda_{+}^{\prime \prime}=(1.213 \pm 0.021) \times 10^{-3}$.

The uncertainty on $\lambda_{+}^{\prime}$ is mainly driven by statistics thus offering good prospects for more accurate results and a better determination of the phase space integral. The correlation between slope and curvature imposed by the dispersion relation [71] used in the $K_{\ell 3}$ dispersive analyses [83, 85, 59, will also be tested.

Moreover, the combination of $\tau, K_{e 3}$ and $K_{\mu 3}$ measurements using the dispersive representation as in Eq. (13) 
and a double-subtracted dispersion relation for the normalized scalar form factor [70,71, could also lead to an improvement in the scalar form factor description. In particular, a more precise determination of the free parameter $\ln C$, where $C=\bar{f}_{0}\left(\Delta_{K \pi}\right)$, is of interest for testing the SM and the presence of right-handed quark or scalar currents [20, 70, 71 .

\subsubsection{Unitarity and universality}

To extract $\left|V_{u s}\right| \times f_{+}(0)$ we use Eq.(2) together with the $S U(2)$-breaking $22,23,24,25$ and long-distance $E M$ corrections to the radiation-inclusive decay rate $[22,23,24,25$ 86. The measured values of $\left|V_{u s}\right| \times f_{+}(0)$ from KLOE are shown in Tab. 5. The five decay modes agree well within

Table 5: Values of $\left|V_{u s}\right| \times f_{+}(0)$ extracted from $K_{l 3}$ decay rates.

\begin{tabular}{ccccc}
\hline$K_{L} e 3$ & $K_{L} \mu 3$ & $K_{S} e 3$ & $K^{ \pm} e 3$ & $K^{ \pm} \mu 3$ \\
\hline $0.2155(7)$ & $0.2167(9)$ & $0.2153(14)$ & $0.2152(13)$ & $0.2132(15)$ \\
\hline
\end{tabular}

the errors and average to $\left|V_{u s}\right| \times f_{+}(0)=0.2157 \pm 0.0006$, with $\chi^{2} / n d f=7.0 / 4(\mathrm{Prob}=13 \%)$. The $0.28 \%$ accuracy of this result has to be compared with the $0.23 \%$ of the world average $\left|V_{u s}\right| \times f_{+}(0)=0.2166 \pm 0.0005$ 61. Significant lepton-universality tests are provided by the comparison of the results from different leptonic channels. Defining the ratio $r_{\mu e}=\left|V_{u s}\right| \times f_{+}(0)_{\mu 3}^{2} /\left|V_{u s}\right| \times f_{+}(0)_{e 3}^{2}$ and using Eq.(2), we have $r_{\mu e}=g_{\mu}^{2} / g_{e}^{2}$, with $g_{\ell}$ the coupling strength at the $W \rightarrow \ell \nu$ vertex. Lepton universality can be then tested comparing the measured value of $r_{\mu e}$ with the SM prediction $r_{\mu e}^{S M}=1$. Averaging charged- and neutral-kaon modes, we obtain $r_{\mu e}=1.000(8)$, to be compared with the results from leptonic pion decays, $\left(r_{\mu e}\right)_{\pi}=1.0042(33)$ [87, and from leptonic $\tau$ decays $\left(r_{\mu e}\right)_{\tau}=1.0005(41)$ [88]. Using the world average of all kaon measurements, $r_{\mu e}=$ 1.004(4) 20 has the same precision than $\tau$ decays.

Using the determination of $\left|V_{u s}\right| \times f_{+}(0)$ from $K_{l 3}$ decays and the value $f_{+}(0)=0.964(5)$ from 38, we get $\left|V_{u s}\right|=0.2237(13)$.

Furthermore, a measurement of $\left|V_{u s}\right| /\left|V_{u d}\right|$ can be obtained from the comparison of the radiation-inclusive decay rates of $K^{ \pm} \rightarrow \mu^{ \pm} \nu(\gamma)$ and $\pi^{ \pm} \rightarrow \mu^{ \pm} \nu(\gamma)$, combined with lattice calculation of $f_{K} / f_{\pi}$ 89. Using $\operatorname{BR}\left(K^{ \pm} \rightarrow\right.$ $\left.\mu^{ \pm} \nu\right)=0.6366(17)$ from KLOE 52 and the lattice result $f_{K} / f_{\pi}=1.189(7)$ from the HP/UKQCD '07 [42, we get $\left|V_{u s}\right| /\left|V_{u d}\right|=0.2323(15)$. This value can be used in a fit together with the measurements of $\left|V_{u s}\right|$ from $K_{l 3}$ decays and $\left|V_{u d}\right|=0.97418(26)$ 90 from superallowed nuclear $\beta$ decays. The result of this fit is $\left|V_{u d}\right|=0.97417(26)$ and $\left|V_{u s}\right|=0.2249(10)$, with $\chi^{2} / n d f=2.34 / 1$ (Prob= $13 \%)$, from which we get $1-\left(\left|V_{u d}\right|^{2}+\left|V_{u s}\right|^{2}+\left|V_{\mathrm{ub}}\right|^{2}\right)=$ $4(7) \times 10^{-4}$ compatible with unitarity at $0.6-\sigma$ level. Using these results, we evaluate $G_{\mathrm{CKM}}=G_{\mathrm{F}}\left(\left|V_{u d}\right|^{2}+\left|V_{u s}\right|^{2}+\right.$ $\left.\left|V_{\mathrm{ub}}\right|^{2}\right)^{1 / 2}=(1.16614 \pm 0.00040) \times 10^{-5} \mathrm{GeV}^{-2}$ which is in perfect agreement with the measurement from the muon lifetime $G_{\mathrm{F}}=(1.166371 \pm 0.000006) \times 10^{-5} \mathrm{GeV}^{-2}$.91] At present, the sensitivity of the quark-lepton universality test through the $G_{\mathrm{CKM}}$ measurement is competitive and even better than the measurements from $\tau$ decays and the electroweak precision tests 92 . Thus unitarity can also be interpreted as a test of the universality of lepton and quark weak couplings to the $W$ boson, allowing bounds to be set on extensions of the SM leading to some kind of universality breaking. For instance, the existence of additional $Z^{\prime}$ gauge bosons, giving different loop-contributions to muon and semileptonic decays, can break gauge universality [12]. The measurement of $G_{\mathrm{CKM}}$ can set constraints on the $Z^{\prime}$ mass which are competitive with direct search at the colliders. When considering supersymmetric extensions, differences between muon and semileptonic decays can arise in the loop contributions from SUSY particles 13,14. The slepton-squark mass difference could be investigated improving present accuracy on the unitarity relation by a factor of $\sim 2-3$.

\subsection{6 $K_{\ell 2}$ and $K_{\ell 3}$ beyond the SM}

The ratio of the $\left|V_{u s}\right|$ values obtained from helicity-suppressed and helicity-allowed kaon modes,

$$
R_{\ell 23}=\frac{\left|V_{u s}\right|\left(K_{\ell 2}\right)}{\left|V_{u s}\right|\left(K_{\ell 3}\right)} \times \frac{V_{u d}\left(0^{+} \rightarrow 0^{+}\right)}{V_{u d}\left(\pi_{\mu 2}\right)}
$$

is equal to unity in the SM. The presence of a scalar current due to a charged Higgs $H^{+}$exchange is expected to lower the value of $R_{\ell 23}$ [93]:

$$
R_{\ell 23}=\left|1-\frac{m_{K^{+}}^{2}}{m_{H^{+}}^{2}}\left(1-\frac{m_{\pi^{+}}^{2}}{m_{K^{+}}^{2}}\right) \frac{\tan ^{2} \beta}{1+0.01 \tan \beta}\right|,
$$

with $\tan \beta$ the ratio of the two Higgs vacuum expectation values in the Minimal Supersymmetric Standar Model, MSSM. The experimental data on $K_{\mu 2}$ and $K_{\ell 3}$ decays are fit in order to evaluate $R_{\ell 23}$, using as external inputs the most recent lattice determinations of $f_{+}(0)$ [38] and $f_{K} / f_{\pi}$ [42, the value of $\left|V_{u d}\right|$ from [90, and $\left|V_{u d}\right|^{2}+\left|V_{u s}\left(K_{l 3}\right)\right|^{2}=$ 1 as a constraint. The result obtained with KLOE data is $R_{\ell 23}=1.008 \pm 0.008$ 61]. From this measurement bounds can be set on the values of the charged Higgs mass and $\tan \beta$ in a region not reachable with the present measurements of the $\mathrm{BR}(B \rightarrow \tau \nu)$ 94. Figure 2 shows the region excluded at $95 \% \mathrm{CL}$ in the charged Higgs mass $m_{H^{ \pm}}$and $\tan \beta$ plane together with the bounds from $\operatorname{BR}(B \rightarrow \tau \nu)$. The measurement of $R_{\ell 23}$ compatible with the SM prediction gives stringent bounds on the presence of charged right-handed currents appearing for instance in some Higgsless low-energy effective theories 71].

\subsubsection{Test of lepton-flavor conservation: $R_{K}$}

KLOE performed a comprehensive study of the process $\Gamma(K \rightarrow e \nu(\gamma))$ based on the complete data set [19. The 


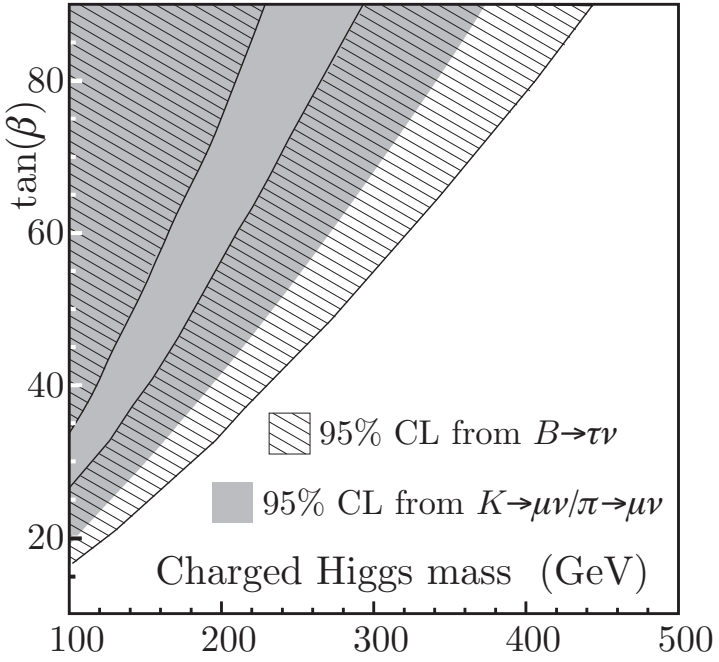

Fig. 2: Excluded region in the $m_{H^{ \pm}}-\tan \beta$ plane from the $R_{\ell 23}$ as measured by KLOE. The region excluded by $B \rightarrow \tau \nu$ is indicated with cross-hatching.

ratio of $\Gamma(K \rightarrow e \nu(\gamma))$ and $\Gamma(K \rightarrow \mu \nu(\gamma))$ decay widths has been measured for photon energies smaller than 10 $\mathrm{MeV}$, without photon detection requirement, $R_{10}=(2.333 \pm$ $\left.0.024_{\text {stat }} \pm 0.019_{\text {stat }}\right) \times 10^{-5}$. The systematic fractional error of $\sim 0.8 \%$, to be compared with the statistical accuracy of $1 \%$, is dominated by the statistics of the controlsample used ( $0.6 \%$ contribution) thus making possible the improvement of the results with larger data samples. The radiation-inclusive ratio $\mathrm{R}_{K}=\left(2.493 \pm 0.025_{\text {stat }} \pm 0.019_{\text {syst }}\right) \times$ $10^{-5}$ has been derived, in excellent agreement with the SM prediction. This result improves the accuracy on $R_{K}$ by a factor of five with respect to the previous world average, putting severe constraints on new physics contributions from MSSM with lepton-flavor violating couplings. The excluded region in the $\tan \beta-m_{H^{ \pm}}$plane as a function of the 1-3 slepton-mass matrix element $\Delta_{13}$ is shown in Fig. 3. Recently, the NA62 collaboration presented a preliminary result $\left[95, R_{K}=\left(2.500 \pm 0.012_{\text {stat }} \pm 0.011_{\text {syst }}\right) \times 10^{-5}\right.$, obtained with $40 \%$ of the sample collected with two dedicated runs at CERN in 2007 and 2008.

The new world average is $R_{K}=(2.498 \pm 0.014) \times 10^{-5}$, with an accuracy of $0.6 \%$.

KLOE-2 with $25 \mathrm{fb}^{-1}$ of integrated luminosity can reach $0.4 \%$ accuracy on $\mathrm{R}_{K}$, a noticeable step forward for the lepton-flavor conservation test.

In the mid-term, the best sensitivity on $\mathrm{R}_{K}$ would be met by the NA62 experiment with the analysis and a severe control over the systematics of the run dedicated to the measurement of the $\operatorname{BR}\left(K^{ \pm} \rightarrow \pi^{ \pm} \nu \bar{\nu}\right)$.

\section{$2.3 \mathrm{KLOE}-2$ prospects on $\left|V_{u s}\right| \times f_{+}(0)$}

KLOE-2 can significantly improve the accuracy on the measurement of $K_{L}, K^{ \pm}$lifetimes and $K_{S} e 3$ branching ratio with respect to present world average 20 with data from the first year of data taking, at KLOE-2/step-0. The

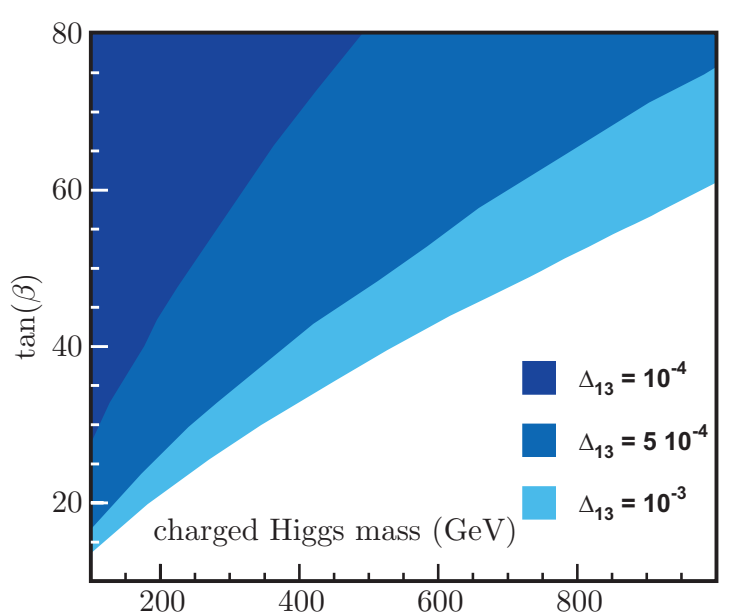

Fig. 3: Excluded region at 95\% C.L. in the $m_{H^{ \pm}}-$ $\tan \beta$ plane from the $R_{\mathrm{K}}$ as measured by KLOE, for $\Delta_{13}=10^{-3}, 0.5 \times 10^{-3}, 10^{-4}$.

Table 6: KLOE-2/step-0 prospects on $\left|V_{u s}\right| \times f_{+}(0)$ extracted from $K_{l 3}$ decay rates.

\begin{tabular}{|c|c|c|c|c|c|}
\hline Mode & $\delta\left|V_{u s}\right| \times f_{+}(0)(\%)$ & $\mathcal{B}$ & $\tau$ & $\delta$ & $\mathrm{I}_{K l}$ \\
\hline$K_{L} e 3$ & 0.21 & 0.09 & 0.13 & 0.11 & 0.09 \\
\hline$K_{L} \mu 3$ & 0.25 & 0.10 & 0.13 & 0.11 & 0.15 \\
\hline$K_{S} e 3$ & 0.33 & 0.30 & 0.03 & 0.11 & 0.09 \\
\hline$K^{ \pm} e 3$ & 0.37 & 0.25 & 0.05 & 0.25 & 0.09 \\
\hline$K^{ \pm} \mu 3$ & 0.40 & 0.27 & 0.05 & 0.25 & 0.15 \\
\hline
\end{tabular}

present $0.23 \%$ fractional uncertainty on $\left|V_{u s}\right| \times f_{+}(0)$ can be reduced to $0.14 \%$ using KLOE present data set together with the KLOE-2/step-0 statistics. The world-average uncertainties on phase space integrals and $K_{L}$ semileptonic BRs 20 have been used in Tab. 6 to summarize the expected accuracy on $\left|V_{u s}\right| \times f_{+}(0)$ for each decay mode and with the contributions from branching ratio, lifetime, $\mathrm{SU}(2)$-breaking and long-distance $E M$ corrections, and phase space integral. Statistical uncertainties on the measurement of BRs and lifetimes have been obtained scaling to the total sample of $7.5 \mathrm{fb}^{-1}$ of integrated luminosity available at the completion of KLOE- $2 /$ step- 0 . The estimate of systematic errors is rather conservative, being based on KLOE published analyses without including any improvement from the detector upgrade.

Lattice QCD simulations have made tremendous progress in the last few years and in near-term the accuracy on $f_{+}(0)$ could reach the $0.1 \%$ level thus allowing $\left|V_{u s}\right|$ to be determined from Tab. 6 with $0.17 \%$ relative error. At this level of precision the accuracy on $\left|V_{u d}\right|$ presently at $0.026 \%$ a6 and dominated by the uncertainty on radiative corrections, becomes the limiting factor in testing CKM unitarity. With $0.02 \%$ accuracy on $\left|V_{u d}\right|$ and with $\left|V_{u s}\right|$ measured at $0.17 \%$, the sensitivity on the unitarity relation would improve by a factor of two reaching the level of a few $10^{-4}$ : a significant opportunity to investi- 
gate beyond-SM models with gauge universality breaking [12, 13, 14, 15.

\section{CPT Symmetry and Quantum Mechanics}

The CPT theorem [97, 98, 99, 100, proves the invariance of physics under combination of the discrete trasformations, $C$ (charge conjugation), $P$ (parity), and $T$ (time reversal). The theorem holds for any quantum field theory formulated on flat space-time assuming i) Lorentz invariance, ii) locality, and iii) unitarity (conservation of probability).

Experimental tests of $C P T$ invariance therefore probe the most fundamental assumptions of our present understanding of particles and their interactions.

The sensitivity of the neutral kaon system to a variety of $C P T$-violating effects makes it one of the best candidates for probing $C P T$ invariance.

A unique feature of the $\phi$-factory is the production of neutral kaon pairs in a pure quantum state so that we can study quantum interference effects and tag pure monochromatic $K_{S}$ and $K_{L}$ beams. The neutral kaon doublet is one of the most intriguing systems in nature. During its time evolution a neutral kaon oscillates to-and-fro between particle and antiparticle states with a beat frequency $\Delta m \approx 5.3 \times 10^{9} \mathrm{~s}^{-1}$, where $\Delta m$ is the mass difference between $K_{L}$ and $K_{S}$. The observation of a rich variety of interference phenomena in the time evolution and decay of neutral kaons is then possible thanks to the special circumstance of a $\Delta m$ value that is half of the $K_{S}$ decay width.

The CPT violation could manifest for instance in conjunction with tiny modifications of the initial correlation of the kaon doublet, with decoherence effects or Lorentz symmetry violations, which in turn might be justified in the context of a quantum theory of gravity.

At KLOE-2 the sensitivity to some observables can meet the level of the Planck scale, $\mathcal{O}\left(m_{K}^{2} / M_{\text {Planck }}\right) \sim$ $2 \times 10^{-20} \mathrm{GeV}$, unreachable in other similar systems, such as $\mathrm{B}$ mesons. As a figure of merit, the fractional mass difference $\left(m_{\mathrm{K}^{0}}-m_{\overline{\mathrm{K}}^{0}}\right) / m_{\mathrm{K}^{0}}$ can be measured to $\mathcal{O}\left(10^{-18}\right)$ while in the neutral $\mathrm{B}$ system experimental accuracy is of the order of $10^{-14}$, and the proton-antiproton mass difference provide a limit at the $10^{-9}$ level [18].

Besides measurements of most of the kaon parameters, the entangled neutral kaons at the $\phi$-factory are also suitable to perform several tests of the foundations of QM, such as state coherence over macroscopic distances, Bohr's complementarity principle, and quantum-erasure and quantum-marking concepts, as explained in Sects. 3.2 3.73 .7 .1

\subsection{The neutral kaon system at the $\phi$ factory}

A pure two-kaon state is produced from $\phi$ decays:

$$
\begin{aligned}
\left|\psi^{-}(t)\right\rangle & =\frac{1}{\sqrt{2}}\left\{\left|K^{0}\right\rangle \otimes\left|\bar{K}^{0}\right\rangle-\left|\bar{K}^{0}\right\rangle \otimes\left|K^{0}\right\rangle\right\} \\
& =\frac{N_{S L}}{\sqrt{2}}\left\{\left|K_{S}\right\rangle \otimes\left|K_{L}\right\rangle-\left|K_{S}\right\rangle \otimes\left|K_{L}\right\rangle,\right.
\end{aligned}
$$

with $N_{S L}=\sqrt{\left(1+\left|\epsilon_{S}\right|^{2}\right)\left(1+\left|\epsilon_{L}\right|^{2}\right)} /\left(1-\epsilon_{S} \epsilon_{L}\right) \simeq 1$ and $\epsilon_{S}=\epsilon+\delta, \epsilon_{L}=\epsilon-\delta$, where $\epsilon$ and $\delta$ denote the $C P$ and $C P T$-violation parameters in the mixing. The state of Eq. (17) is antisymmetric with respect to permutations of kaons, and exhibits maximal entanglement. decay mechanism into a Lindblad operator of a master equation. The maximal entanglement of kaon pairs as in Eq.117) has been observed in the $\phi \rightarrow K_{S} K_{L} \rightarrow \pi^{+} \pi^{-} \pi^{+} \pi^{-} 101$ by the KLOE collaboration in year 2005. Since then, more data (a total of $1.7 \mathrm{fb}^{-1}$ ) and improvements in the analysis procedure have brought to the results on several decoherence and $C P T$-violating parameters [102] that will be presented in this section.

The selection of the signal requires two vertices, each with two opposite-curvature tracks inside the drift chamber, with an invariant mass and total momentum compatible with the two neutral kaon decays. The resolution on $\Delta t$, the absolute value of the time difference of the two $\pi^{+} \pi^{-}$decays, benefits of the precision momentum measurements and of the kinematic closure of the events. The $\Delta t$ distribution can be fitted with the function:

$$
\begin{array}{r}
I\left(\pi^{+} \pi^{-}, \pi^{+} \pi^{-} ; \Delta t\right) \propto e^{-\Gamma_{L} \Delta t}+e^{-\Gamma_{S} \Delta t} \\
-2 e^{-\frac{\left(\Gamma_{S}+\Gamma_{L}\right)}{2} \Delta t} \cos (\Delta m \Delta t),
\end{array}
$$

evaluated from QM, vanishing at $\Delta t=0$ 103 for the complete antisymmetry of the state of Eq. (17). The fit of the $\Delta t$ distribution is performed taking into account resolution and detection efficiency, the background from coherent and incoherent $K_{S}$-regeneration on the beam pipe wall, and the small contamination from the non-resonant $e^{+} e^{-} \rightarrow \pi^{+} \pi^{-} \pi^{+} \pi^{-}$channel. The result obtained fixing $\Delta m, \Gamma_{S}$ and $\Gamma_{L}$ to the Particle Data Group (PDG) values [18, is shown in Fig. 4 .

Different hypotheses on decoherence and $C P T$-violating phenomena are expressed by different modifications of the function of Eq.(18). The modified expressions have been then used to obtain the best values of the QM- and CPTviolating parameters $\left(\zeta_{K_{S} K_{L}}, \zeta_{K^{0} \bar{K}^{0}}, \gamma, \operatorname{Re} \omega, \operatorname{Im} \omega, \Delta a_{X}\right.$, $\left.\Delta a_{Y}, \Delta a_{Z}\right)$ presented in the following subsections.

\subsection{Test of quantum coherence}

The kaon states evolve exponentially in time according to the Wigner-Weisskopf approximation with an effective Hamiltonian consisting of the Hermitean and antiHermitean components. Time evolution can be also described using a Hermitean Hamiltonian and the master equation in the Kossakowski-Lindblad form of Ref. [104.

Due to conservation of the quantum numbers, it is safe to assume that the initial state is indeed the maximally entangled state given by Eq.(17). However, it is by no means proven that the assumed quantum mechanical time evolution (the Wigner-Weisskopf approximation) is the correct one. It may well be that the kaon pair undergoes decoherence effects, i.e., the initially pure state would become mixed for $t>0$.

Decoherence may result from fundamental modifications of QM and may be traced back to the influence of 


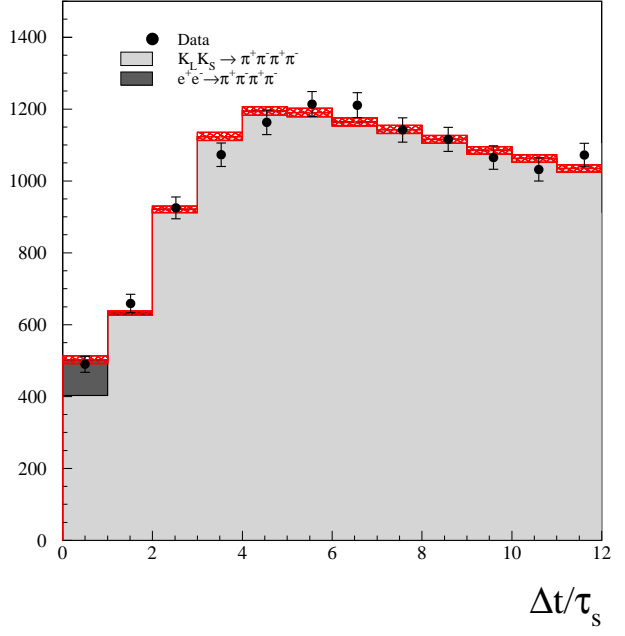

Fig. 4: Number of events as a fuction of the difference in the decay time of the two $\pi^{+} \pi^{-}$vertices, in $\tau_{S}$ units. Bin width is 1 . The measured $I\left(\pi^{+} \pi^{-}, \pi^{+} \pi^{-} ; \Delta t\right)$ distribution is fitted: black points with errors are data and the fit result is shown by the histogram.

quantum gravity 105 106 - quantum fluctuations in the space-time structure on the Planck mass scale - or to dynamical state reductions 107, 108.

Thus from the experimental study of the decoherence we can answer to:

- How accurately is the QM interference term verified by the experimental data?

- Is the purity of the two-kaon state maintained for times $t>0$ ?

The general formalism of open quantum systems, that means a master equation of type Lindblad 109 and GoriniKossakowski-Sudarshan [110, derived under rather general assumptions, allows the study of different scenarios of decoherence without modeling the environment explicitly. Such scenarios have been proposed and, interestingly, a direct relation between the decoherence parameter and the loss of entanglement has been established 103. The decoherence parameter depends on the basis used to describe time evolution of the kaon doublet.

A simpler but very effective approach to treat decoherence is based on an idea of Schrödinger [11] and Furry [112, proposed already in 1935. They raised the question whether an initially entangled state can spontaneously factorize into product states, with a basis-dependent spontaneous factorization. We can quantify the effect by multiplying the QM interference term by a factor $(1-\zeta)$, where $\zeta$ represents the decoherence parameter 113,114 and varies between $0 \leq \zeta \leq 1$, the limits of QM and spontaneous factorization.

KLOE has provided the first experimental evidence for quantum interference in the $C P$-suppressed process $\phi \longrightarrow K_{S} K_{L} \longrightarrow \pi^{+} \pi^{-} \pi^{+} \pi^{-}$obtaining for the decoherence parameter $\zeta$ in $K_{S} K_{L}$ and $K^{0} \bar{K}^{0}$ bases [102,101]:

$$
\begin{aligned}
\zeta_{K_{S} K_{L}} & =0.003 \pm 0.018_{\text {stat }} \pm 0.006_{\text {sys }} \\
\zeta_{K^{0} \bar{K}^{0}} & =\left(1.4 \pm 9.5_{\text {stat }} \pm 3.8_{\text {sys }}\right) \cdot 10^{-7} .
\end{aligned}
$$

Since decoherence in the $K^{0} \bar{K}^{0}$ basis would result in the $C P$-allowed $K_{S} K_{S} \rightarrow \pi^{+} \pi^{-} \pi^{+} \pi^{-}$decays, the sensitivity for the parameter $\zeta_{K^{0} \bar{K}^{0}}$ is naturally much larger than for $\zeta_{K_{S} K_{L}}$. The KLOE results represent a large improvement with respect to the analysis published in year 1998 [114, based on the results of the CPLEAR experiment [115]: $\zeta_{K_{S} K_{L}}=0.13 \pm 0.16, \zeta_{K^{0} \bar{K}^{0}}=0.4 \pm 0.7$.

The upper limits can be definitely improved with KLOE2 , meeting the scale of quantum gravity and dynamical state reduction. The decoherence parameter $\zeta$ is a timeintegrated value and in the framework of open quantum systems we have the following relation [103]:

$$
\zeta\left(\min \left\{t_{l}, t_{r}\right\}\right)=1-e^{-\lambda \min \left\{t_{l}, t_{r}\right\}},
$$

where $\lambda$ is a constant representing the strength of the interaction with the environment. Since we are interested in the decoherence (or entanglement loss) of the two-kaon system, we consider time evolution of the total state up to the minimum of the two times $t_{l}, t_{r}$, when the measurements are performed on the left- and right-hand side. After the first measurement, the two-kaon state collapses and the remaining one-kaon state evolves according to QM until it is measured too.

The parameter $\lambda$ is considered to be the fundamental one. A comparison with other meson systems is of great interest to understand how universal $\lambda$ is, how does it depend on the kind of environment and how does it typically scale with the mass of the system.

The Belle collaboration, at the KEK B-factory in Japan, has analyzed data from the two-B-meson system obtaining, in the flavour basis [116], $\zeta_{B^{0}, \bar{B}^{0}}=0.029 \pm 0.057$.

A precision measurement of the decoherence parameter can provide stringent upper bounds that in turn can rule out several sources of decoherence, using data from neutral mesons and also from photon, neutron, and molecular systems.

\section{3 $C P T$ violation from quantization of space-time}

The study of $C P T$ symmetry at KLOE-2 can contribute to the research program of Quantum Gravity Phenomenology [117.

The $C P T$ and Lorentz symmetry are to be placed under scrutiny whenever quantization of the space-time is adopted. The simplest line of reasoning that supports this thesis takes as starting point the duality relation between the (smooth, classical, Riemannian) light-cone structure of Minkowski space-time and the (classical, Lie-algebra) structure of Poincare' symmetries. This duality between space and its symmetry algebra is rather rigid and very sensitive to the introduction of new elements in the structure of the space. Galilei space-time and the Galilei algebra are linked by an analogous duality relation, and in 
going from Galilei to Minkowski space-time, thus adding the light-cone structure, one must replace the Galilei symmetries with the Poincare' ones. Similarly, we found that the additional space-time elements introduced by different proposals of the space-time quantization typically affect also the description of the symmetries. And indeed over the last decade or so the scenarios with broken Poincare's symmetries [118, 119, 120, 121 or those alternatively introducing the novel concept of deformed Poincare's symmetries [122 123, 124, have experienced a fast-growing popularity in the quantum-gravity studies.

These studies on Poincare's transformations have often implications on the CPT symmetry, producing scenarios with broken $C P T$ invariance 125 or alternatively with a deformed concept of CPT at the Planck-scale [126, 127].

Several tests of quantum-spacetime-inspired scenarios with broken or deformed Poincare's symmetries have been proposed and are being performed by astrophysics experiments [118, 120, 121, 128, 129, 130.

The sensitivity reach of these studies is very promising although the control on possible competitive phenomena is typically rather limited. As a result of our lack of knowledge, the experimental bounds on the parameters of interest are often conditional [117 and rely on some assumptions on the properties of the astrophysical systems. For this reason the quantum gravity community still looks with great interest at the results from controlled laboratory experiments such as KLOE-2.

A key characteristic of the effects expected from spacetime quantization at the Planck scale is the peculiar dependence of the effects on energy. For example, the most studied effects of violation of classical Poincare' symmetry are propagation effects, often codified with modifications of the dispersion relation:

$$
m^{2} \simeq E^{2}-\mathbf{p}^{2}+\eta \mathbf{p}^{2} \frac{E^{n}}{E_{p}^{n}}+\ldots
$$

where $E_{p}$ denotes the Planck scale, the parameter $\eta$ sets the magnitude of the effect, and the integer $n$ (usually $n=1$ or $n=2$ ) fixes the dependence on energy (and the Planck-scale) of the leading Planck-scale effect.

The possibility to formulate these effects within effective field theories is not always viable. Some aspects of the space-time noncommutativity introduce non-analytic features that must be handled with care when attempting to reformulate quantum space-time properties effectively as novel properties of an ordinary field theory in a classical space-time. In cases where an effective-field-theory formulation appears to be viable these effects end up requiring the introduction of non-renormalizable terms in the Lagrangian density, such as dimension-5 and dimension- 6 operators (in the case of theories formulated in 4 spacetime dimensions).

Since the whole quantum gravity problem is plagued by objective difficulties at the level of perturbative renormalizability, the quantum-gravity motivation for this type of studies of space-time quantization conceptually renders formalization in terms of nonrenormalizable field theories rather natural.
On the other hand, this nonrenormalizability still represents a serious challenge for the efforts on the phenomenology side. A study of the implications for KLOE-2 of these specific quantum-spacetime Poincare'-violation effects is presently under way 131 .

The prospects to investigate with KLOE-2 some scenarios for $C P T$ symmetry emerging from quantum spacetime studies are even more interesting. While experimental tests of the Poincare' or Lorentz symmetry in astrophysics are rather powerful, the controlled, laboratory experiments are essential for the $C P T$ tests.

Since space-time quantization is expected to imply nontrivial effects on the coherence of multiparticle states, the tests of CPT invariance at KLOE-2 based on the coherent neutral kaon system from $\phi$ decay, are particularly significant from a quantum space-time perspective.

In our current (pre-quantum-gravity) theories one obtains multiparticle states from single-particle states by a standard use of the trivial tensor product of Hilbert spaces, but there is theoretical evidence that this recipe might not be applicable in a quantum-spacetime environment.

A valuable phenomenological formulation of this intuition has been recently proposed in Ref. 132 and is presented in Sect. 3.4

A robust derivation of the properties of multiparticle states within specific quantum space-time proposals is still obscured by the mathematical complexity of the theoretical framework. Nevertheless, recent preliminary results 127. on theories formulated in noncommutative spacetime are promising for providing in not-too-far future a valuable theory of the multiparticle systems to be tested with KLOE-2.

\subsection{CPT violation and decoherence}

In this and following subsections we shall concentrate on the possible breakdown of the $C P T$ symmetry and in testing it with the entangled states of neutral kaons at the $\phi$-factory. In general, there are two ways by which $C P T$ breakdown is encountered in a quantum gravity model.

The first is through the non-commutativity of a welldefined quantum mechanical CPT operator with the Hamiltonian of the system. This is the breakdown of CPT symmetry dealt with in standard Lorentz-violating Extensions of the Standard Model (SME) [133, 134, 135.

In the second way, the $C P T$ operator is ill-defined as a quantum mechanical operator, but in a perturbative sense to be described below. This ill-defined operator is a consequence of the foamy structure [136] of space-time, whereby the quantum metric fluctuations at Planck scales induce quantun decoherence of matter. The particle field theoretical system is an open quantum mechanical system interacting with the environment of quantum gravity. The reason for ill-defined CPT operator in such cases is of more fundamental nature than the mere non-commutativity of this operator with the local effective Hamiltonian of the matter system in Lorentz-symmetry violating SME models. Quantum-gravity induced decoherence is in operation 
and the very concept of a local effective Lagrangian may itself break down. R. Wald [137] has elegantly argued, based on elementary quantum mechanical analysis of open systems, that the CPT operator is ill-defined for systems which exhibit quantum decoherence, that is they are characterised by an evolution of initially pure quantum mechanical states to mixed ones. This was interpreted as a microscopic time arrow in quantum gravitational media which induce such decoherence. Hence such open systems are characterised by intrinsic CPT violation, a terminology we shall use from now in order to describe this particular type of CPT symmetry breakdown.

Lindblad Parametrizations of Decoherent Evolution in Quantum Gravity: in the past [138, 139, quantum gravity decoherence has been paramaterised in the neutral kaon system by means of a Lindblad-type evolution of the reduced density matrix for kaons, which satisfies energy conservation on average, positivity of the density matrix and probability conservation. In its simplest form, taking into account additional constraints relevant for neutral kaon physics (such as $\Delta S=\Delta Q$ rule), the relevant evolution operator for the density matrices of a single neutral kaon state is given by [138, 139]:

$\partial_{t} \rho=i[\rho, H]+\delta \not H \rho$,
where $H_{\alpha \beta}=\left(\begin{array}{cccc}-\Gamma & -\frac{1}{2} \delta \Gamma & -\operatorname{Im} \Gamma_{12} & -\operatorname{Re} \Gamma_{12} \\ -\frac{1}{2} \delta \Gamma & -\Gamma & -2 \operatorname{Re} M_{12} & -2 \operatorname{Im} M_{12} \\ -\operatorname{Im} \Gamma_{12} & 2 \operatorname{Re} M_{12} & -\Gamma & -\delta M \\ -\operatorname{Re} \Gamma_{12} & -2 \operatorname{Im} M_{12} & \delta M & -\Gamma\end{array}\right)$, and $\delta H_{\alpha \beta}=\left(\begin{array}{cccc}0 & 0 & 0 & 0 \\ 0 & 0 & 0 & 0 \\ 0 & 0 & -2 \alpha & -2 \beta \\ 0 & 0 & -2 \beta & -2 \gamma\end{array}\right)$. Positivity of $\rho$ requires: $\alpha, \gamma>0, \quad \alpha \gamma>\beta^{2}$. Notice that $\alpha, \beta, \gamma$ violate both $C P T$, due to their decohering nature [137, and $C P$ symmetry.

However, as pointed out in Ref. 140, although the above parametrization guarantees a positive-definite density matrix for single kaon states, this is not true for entangled kaon states, as those produced at the $\phi$-factory. For entangled states, the requirement of complete positivity implies different parametrizations for the foam effects. For instance, if one chooses the above-mentioned parametrization of Refs. [138, 139], for consistency with the requirement of universal action of quantum gravity on both single-particle and entangled states, then in the latter case, complete positivity is guaranteed only if the further conditions $\alpha=\gamma$ and $\beta=0$ are imposed. The latest measurement of $\gamma$ from KLOE yields the result [102, 101:

$$
\gamma=\left(0.7 \pm 1.2_{\text {stat }} \pm 0.3_{\text {syst }}\right) \times 10^{-21} \mathrm{GeV},
$$

consistent with zero. Nevertheless, the experiment can measure all three decoherence parameters $\alpha, \beta, \gamma$ and hence one can continue using the full set 141] when discussing neutral kaon effects at the $\phi$-factory. In this way one can provide a formalism for testing experimentally the assumption of complete positivity, which notably may not be a property of quantum gravity.
Decoherence, Ill-defined CPT Operator and the $\omega$-effect in Entangled States: in addition to the decoherence evolution parameters $\alpha, \beta, \gamma$, in entangled states one may have 132,142 some other effects associated with the illdefined nature of the CPT operator in decoherent models of quantum gravity. As a result of the weak nature of quantum gravitational interactions, this ill-defined nature of CPT operator is perturbative in the sense that the antiparticle state still exists, but its properties, which under normal circumstances would be connected by the action of this operator, are modified [132,142. The modifications can be perceived 143,144] as a result of the dressing of the (anti-)particle states by perturbative interactions expressing the effects of the medium.

As argued in Refs. 132,142, the perturbatively ill-defined nature of the CPT operator implies modified EinsteinPodolsky-Rosen (EPR) correlation among the entangled states in meson factories, which are uniquely associated with this effect, termed as $\omega$-effect.

Experimental Signatures of the $\omega$-effect in kaons at the $\phi$ - factory: if $C P T$ is intrinsically violated, in the sense of being not well-defined due to decoherence [137, the neutral mesons $K^{0}$ and $\bar{K}^{0}$ should no longer be treated as particles-antiparticles states. As a consequence [132,142, the initial entangled state at the $\phi$-factory assumes the form:

$$
\begin{gathered}
|\psi\rangle=\mathcal{N}\left\{\left\{\left|K^{0}\right\rangle_{\mathbf{k}} \otimes\left|\bar{K}^{0}\right\rangle_{-\mathbf{k}}-\left|\bar{K}^{0}\right\rangle_{-\mathbf{k}} \otimes\left|K^{0}\right\rangle_{\mathbf{k}}\right\}+\right. \\
\left.\omega\left\{\left|K^{0}\right\rangle_{\mathbf{k}} \otimes\left|\bar{K}^{0}\right\rangle_{-\mathbf{k}}+\left|\bar{K}^{0}\right\rangle_{-\mathbf{k}} \otimes\left|K^{0}\right\rangle_{\mathbf{k}}\right\}\right\}
\end{gathered}
$$

where $\omega=|\omega| e^{i \Omega}$ is a complex parameter, related to the intrinsic $C P T$-violating modifications of the EPR correlations. The $\omega$ parameter controls perturbatively the amount of contamination of the right-symmetry state by the wrong-symmetry state. The appropriate observable (Fig. 5) is the intensity [132, 142]:

$$
I(\Delta t)=\int_{\Delta t \equiv\left|t_{1}-t_{2}\right|}^{\infty}|A(X, Y)|^{2}
$$

with $A(X, Y)$ the decay amplitude of the state $\psi$, where one kaon decays to the final state $X$ at $t_{1}$ and the other to $Y$ at time $t_{2}$.

At the $\phi$-factory there is a particularly good channel, with $\pi^{+} \pi^{-}$as final decay products for both kaons, which enhances the sensitivity to the $\omega$-effect by three orders of magnitude. This is due to the fact that this channel is $C P$ forbidden for the right symmetry, whereas is $C P$-allowed for the wrong symmetry. Then, the relevant terms [132, 142 in the intensity $I(\Delta t)$ (Fig. 5) contain the combination $\omega /\left|\eta_{+-}\right|$, where $\eta_{+-}$is the $C P$-violating amplitude for the $\pi^{+} \pi^{-}$states, which is of the order $10^{-3}$. The KLOE experiment has obtained the first measurement of the $\omega$ parameter [102,101]:

$$
\begin{aligned}
& \operatorname{Re}(\omega)=\left(-1.6_{-2.1_{\text {stat }}^{+3.0}} \pm 0.4_{\text {syst }}\right) \times 10^{-4}
\end{aligned}
$$

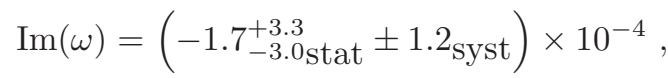




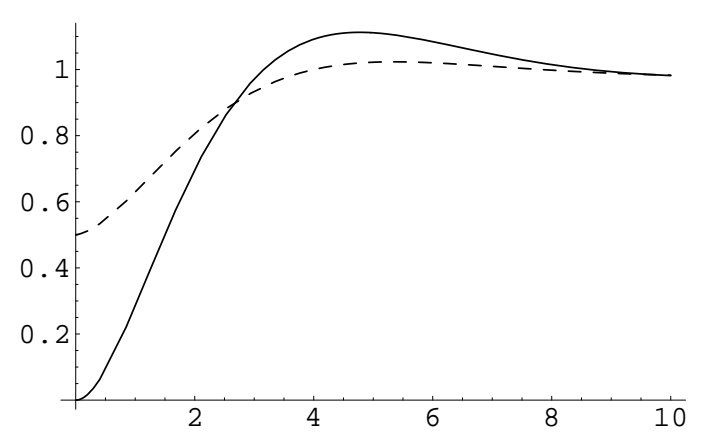

Fig. 5: The intensity $I(\Delta t)$ in arbitrary units, with $\omega=$ $|\omega| e^{i \Omega}=0$ (solid line) and $\omega=\left|\eta_{+-}\right| e^{i\left(\phi_{+-}-0.16 \pi\right)}$ (dashed line) 132,142.

that is $|\omega|<1.0 \times 10^{-3}$ at $95 \%$ C.L.. At least one order of magnitude improvement is expected with KLOE-2 at the upgraded DA $\Phi$ NE [102,101].

This sensitivity is not far from certain models of space time foam leading to $\omega$-like effects [143, 144]. Indeed, in such models, inspired by string theory, the $\omega$-effect is the result of local distortions of space-time in the neighborhood of space-time defects, which interact -via topologically non-trivial interactions (string capture / splitting)only with electrically neutral matter string states, due to electric charge conservation. The recoil of the Planck-mass defect results in metric deformations along the direction of motion of the string state, $g_{0 i} \sim \Delta k^{i} / M_{P}=\zeta k^{i} / M_{P}$, where $\Delta k^{i}=\zeta k^{i}$ denotes the momentum transfer of the matter state. On average, $\left\langle\zeta k^{i}\right\rangle=0$, so Lorentz invariance holds macroscopically, but one has non trivial quantum fluctuations $\left\langle\zeta^{2} k_{i} k_{j}\right\rangle \propto \delta_{i j} \bar{\zeta}^{2}|\mathbf{k}|^{2}$. It can be shown [143. 144 that as a result of such stochastic effects of the space time foam, neutral entangled states - such as the ones in meson factories - exhibit $\omega$-like effects, with the order of magnitude estimate: $|\omega|^{2} \sim \frac{|\mathbf{k}|^{4} \bar{\zeta}^{2}}{M_{P}^{2}\left(m_{1}-m_{2}\right)^{2}}$, where $m_{i}$, $i=1,2$ are the masses of the (near degenerate) mass eigenstates. At the DA $\Phi$ NE energy, for instance, $|\omega| \sim 10^{-4}|\bar{\zeta}|$, which lies within the sensitivity of the facility for values of the average momentum transfer $\bar{\zeta}>10^{-2}$ (which may be expected on account of naturalness), although this is actually a number that depends on the microscopic quantum theory of gravity, and hence is still elusive. Nevertheless, in some concrete string-theory-inspired foam models examined in Ref. [144, this parameter is of the order $|\zeta| \sim \frac{m_{1}^{2}+m_{2}^{2}}{k^{2}}$, and hence such models are in principle falsifiable at KLOE-2.

We close by mentioning that the $\omega$-effect can be disentangled 132,142 experimentally from both, the $\mathrm{C}($ even) background - by means of different interference with the $\mathrm{C}$ (odd) resonant contributions - and the decoherent evolution effects of space-time foam 138,139, due to different structures in the relevant evolution equations of the reduced density matrices.

\section{5 $C P T$ violation and Lorentz-symmetry breaking}

This subsection addresses the prospects of searching for a different type of $C P T$ violation at KLOE-2: as opposed to the situation described in Sec. 3.4. observable systems are taken to be governed by the standard laws of quantum mechanics including unitary time evolution. It is further assumed that possible $C P T$-violating effects beyond the Standard Model (SM) and General Relativity (GR) can be described at low energies within the framework of effective field theory (EFT) 1 This starting point together with a few mild mathematical assumptions immediately implies that $C P T$ violation is necessarily accompanied by Lorentz-symmetry breakdown [134,135, a result sometimes called the anti- $C P T$ theorem. In what follows, $C P T$ and Lorentz-symmetry breakdown therefore can be treated on an equal footing. Note that the converse of this statement, namely that Lorentz-symmetry breakdown always comes with $C P T$ violation, is not true in general. In the following we will discuss how the connection between $C P T$ and Lorentz-symmetry breaking can be exploited for $C P T$-violation searches in neutral meson systems.

The above ideas form the foundation for the construction of the SME (c.f. Sect 3.4) 145, 146, 147]: one starts with the usual Lagrangians $\mathcal{L}_{\mathrm{SM}}$ and $\mathcal{L}_{\mathrm{GR}}$ for the $\mathrm{SM}$ and GR, respectively, and then adds $C P T$ - and Lorentzviolating corrections $\delta \mathcal{L}_{\mathrm{CPTV} / \mathrm{LV}}$ :

$$
\mathcal{L}_{\mathrm{SME}}=\mathcal{L}_{\mathrm{SM}}+\mathcal{L}_{\mathrm{GR}}+\delta \mathcal{L}_{\mathrm{CPTV} / \mathrm{LV}}
$$

Here, $\mathcal{L}_{\mathrm{SME}}$ denotes the SME Lagrangian. The corrections $\delta \mathcal{L}_{\mathrm{CPTV} / \mathrm{LV}}$ are constructed by contracting conventional SM or GR fields of arbitrary mass dimension with $C P T$ and Lorentz-symmetry-breaking nondynamical vectors or tensors to form quantities that transform as scalars under coordinate changes. A SME term may be assumed in the form:

$$
\delta \mathcal{L}_{\mathrm{CPTV} / \mathrm{LV}} \supset-a_{\mu} \bar{\psi} \gamma^{\mu} \psi,
$$

where $\psi$ is a SM fermion and $a_{\mu}$ controls the extent of $C P T$ - and Lorentz-symmetry- violation. We remark that $a_{\mu}$ depends on the fermion species. It will be explained below that neutral-meson oscillations are sensitive to this particular type of coefficient.

SME coefficients for CPT and Lorentz-symmetry breakdown, like $a_{\mu}$ in (28), are assumed to be caused by more fundamental physics possibly arising at the Planck-scale. On phenomenological grounds, these coefficients must be minuscule, and theoretical considerations suggest that they might be suppressed by some power of the Planck mass. Mechanisms for generating SME coefficients can be accommodated in various approaches to physics beyond the SM and GR, such as string theory [148, 149, 150, various space-time-foam models [11, 151, 152, non-commutative

\footnotetext{
1 The SM and GR themselves are widely believed to be EFTs arising from underlying physics. It would then seem contrived to presume that leading-order CPT-violating corrections lie outside EFT. Moreover, EFT has been successful in physics, and remains applicable even in the presence of discrete backgrounds, such as in condensed-matter systems.
} 
field theory [153], and cosmologically varying scalars 154 155, 156. In this sense, the SME is well motivated.

A number of studies have provided the SME with a firm theoretical foundation [157, 158, 159, 160, 161, 162, 163, 164. To date, the SME has been employed to identify ultrahigh-precision CPT and Lorentz-symmetry tests and has provided the basis for the analysis of numerous experimental CPT- and Lorentz-violation searches in astrophysics 165, 166], AMO (atomic, molecular, and optical) physics [167, 168, 169, at colliders 170, 171, 172, 133, 9, 60, 173, 174, 175, and with gravity 176]. For practical reasons, most of these observational results constrain SME coefficients for stable or quasistable particles (i.e., photons, electrons, protons, and neutrons) 177. Experimental tests of $C P T$ and Lorentz symmetry for unstable particles are more difficult, and the majority of the corresponding SME coefficients remain unbounded. The extraction of experimental limits for such unstable particles therefore assumes particular urgency. Neutral-meson interferometry is currently the only feasible method to determine Planck-scale limits on $C P T$ breakdown in the quark sector.

The next step is to employ the SME to make predictions for neutral meson interferometry. To this end, recall that an effective quantum-mechanical description of such a meson system is provided by a simple $2 \times 2$ Hamiltonian $\Lambda$. Indirect $C P T$ violation in this system occurs if and only if the difference $\Delta \Lambda \equiv \Lambda_{11}-\Lambda_{22}$ of the diagonal elements of $\Lambda$ is nonzero. It follows that $\Lambda$ contains two real parameters for $C P T$ breakdown. The $w \xi$ formalism provides a convenient parametrization of $\Lambda$ that is independent of phase conventions, valid for arbitrary-size $C P T$ and $T$ breaking, model independent, and expressed in terms of mass and decay rates insofar as possible [178. In this formalism, the $C P T$ violation determined by $\Delta \Lambda$ is controlled by the parameter $\xi_{K}$. On the other hand, the usual parametrization of $\Lambda$ depends on the phase convention and can be applied only if both $C P T$ and $T$ violation are small. In this conventional parametrization, $C P T$ violation is governed by the coefficient $\delta_{K}$. The relation between $\xi_{K}$ and $\delta_{K}$ is given by $\xi_{K} \approx 2 \delta_{K}$.

The remaining task is now to determine $\Delta \Lambda$ in terms of SME coefficients. This is most conveniently achieved with perturbative methods: the leading-order corrections to $\Lambda$ are expectation values of $C P T$ - and Lorentz-violating interactions in the Hamiltonian evaluated with the unperturbed meson wave functions $\left|P^{0}\right\rangle,\left|\overline{P^{0}}\right\rangle$. Note that the hermiticity of the perturbation Hamiltonian leads to real contributions [179, 180]:

$$
\Delta \Lambda \approx \beta^{\mu} \Delta a_{\mu},
$$

where $\beta^{\mu}=\gamma(1, \beta)$ is the four-velocity of the meson state in the observer frame. In this equation, we have defined $\Delta a_{\mu}=r_{q_{1}} a_{\mu}^{q_{1}}-r_{q_{2}} a_{\mu}^{q_{2}}$, where $a_{\mu}^{q_{1}}$ and $a_{\mu}^{q_{2}}$ are coefficients for $C P T$ - and Lorentz-invariance violation for the two valence quarks in the $P^{0}$ meson. These coefficients have mass dimension one, and they arise from Lagrangian terms of the form (28), more specifically $-a_{\mu}^{q} \bar{q} \gamma^{\mu} q$, where $q$ denotes the quark flavor. The quantities $r_{q_{1}}$ and $r_{q_{2}}$ characterize normalization and quark-binding effects.
The result (29) illustrates that among the consequences of $C P T$ breakdown are the 4-velocity and the 4-momentum dependence of observables. This dependence on the direction is associated with Lorentz-invariance violation, in accordance with the above considerations involving the anti-CPT theorem. It thus becomes apparent that the standard assumption of a constant parameter $\delta_{K}$ for $C P T$ violation is incompatible with unitary quantum field theory. More specifically, the presence of the 4-velocity in Eq. (29) implies that CPT observables will typically vary with the magnitude and orientation of the meson momentum. Additional effect is the motion of the laboratory: the $C P T$ - and Lorentz-violating SME coefficient $\Delta a_{\mu}$ is taken as space-time constant, and it is conventionally specified in the Sun-centered celestial equatorial frame. But Earthbased laboratories move with respect to this frame so sidereal (and in principle seasonal) variations of observables are possible. Thus, time, direction, and momentum binning is required for $C P T$ tests in neutral-mesons systems. Moreover, different laboratories have different orientations in the celestial equatorial frame and hence can be sensitive to different components of $\Delta a_{\mu}$.

Another important fact is that the $C P T$ violation in each of the neutral-meson systems (e.g., $K, D, B_{d}, B_{s}$ ) may be governed by a different $\Delta a_{\mu}$. Because of the distinct masses and decay rates, the physics of each system is distinct. Since each $\Delta a_{\mu}$ contains four components, a complete experimental study of $C P T$ breaking requires four independent measurements in each neutral-meson system.

In a high-energy fixed-target experiment, the momenta of the mesons are approximately aligned with the beam direction so that only the time stamp of the event is needed for directional information. These measurements typically involve uncorrelated mesons, a fact that further simplifies their conceptual analysis. From the decomposition $\beta_{\mu} \Delta a^{\mu}=\left(\beta^{0} \Delta a^{0}-\Delta \mathbf{a}_{\|} \cdot \boldsymbol{\beta}_{\|}\right)-\left(\Delta \mathbf{a}_{\perp} \cdot \boldsymbol{\beta}_{\perp}\right)$, where $\|$ and $\perp$ are taken with respect to the Earth's rotation axis, it is apparent that all four components of $\Delta a_{\mu}$ can be determined: the $\perp$ components via their sidereal variations and the sidereally constant piece in the first parentheses via its dependence on the momentum. However, the variation of $|\boldsymbol{\beta}|$ with the energy is tiny, so it is difficult to disentangle the individual components $\Delta a^{0}$ and $\Delta \mathbf{a}_{\|}$. These ideas have been employed in experiments with the $K$ and $D$ systems. For the $K$ system, a linear combination of $\Delta a_{0}$ and $\Delta a_{Z}$ as well as a linear combination of $\Delta a_{X}$ and $\Delta a_{Y}$ have been bounded to about $10^{-20} \mathrm{GeV}$ and $10^{-21} \mathrm{GeV}$, respectively 170,171 . For the $D$ system, the FOCUS experiment has constrained both a linear combination of $\Delta a_{0}$ and $\Delta a_{Z}$ as well as $\Delta a_{Y}$ to roughly $10^{-16}$ $\mathrm{GeV}$ 172.

CPT tests are also possible with correlated mesons at a symmetric collider, a set-up relevant for the KLOE-2 experiment. Since kaons at the $\phi$-factory are monoenergetic, the analysis of energy dependence can not be pursued; nevertheless such a set-up has other crucial benefits: the wide angular distribution and the correlation of the mesons allows the extraction of limits on all four components of $\Delta a_{\mu}$. 
Searches for $C P T$ violation at symmetric-collider experiments with correlated kaons require analyses of the double-decay rate of the quarkonium state for various final states $f_{1}, f_{2}$. With sufficient experimental resolution, the dependence of certain decays on the two meson momenta $\mathbf{p}_{1}, \mathbf{p}_{2}$ and on the sidereal time $t$ can be extracted by appropriate data binning and analysis. Note that different asymmetries can be sensitive to distinct components of $\Delta \Lambda$.

Consider the case of double-semileptonic decays of correlated kaon pairs. Assuming the $\Delta S=\Delta Q$ rule, it can be shown that the double-decay rate $R_{l^{+} l^{-}}$depends on the ratio:

$$
\left|\frac{\eta_{l^{+}}}{\eta_{l^{-}}}\right| \approx 1-\frac{4 \operatorname{Re}\left(i \sin \phi_{S W} e^{i \phi_{S W}}\right)}{\Delta m} \Delta \Gamma(\mathbf{p}) \Delta a_{0} .
$$

Here, $\phi_{S W} \equiv \tan ^{-1}\left(2 \Delta m /\left(\Gamma_{S}-\Gamma_{L}\right)\right)$ is the superweak angle and $\Delta \Gamma(\mathbf{p})$ stands for the difference of the $K_{L}$ and $K_{S}$ decay widths. Note the absence of all angular and time dependence in Eq. (30). This fact arises because for a symmetric collider the relation $\boldsymbol{\beta}_{\mathbf{1}} \cdot \Delta \mathbf{a}=-\boldsymbol{\beta}_{\mathbf{2}} \cdot \Delta \mathbf{a}$ holds, which leads to a cancellation between the effects from each kaon. KLOE has exploited this idea to place limits on $\Delta a_{0}$ 133, 9, 60:

$$
\Delta a_{0}=(0.4 \pm 1.8) \times 10^{-17} \mathrm{GeV} .
$$

There are also other double-decay channels suitable for $C P T$ tests, in which there is no longer a cancellation of the spatial contributions of $\Delta a_{\mu}$. Mixed double decays, in which only one of the two kaons has a $\xi_{K}$-sensitive mode, are one example. Another possibility is the decay $K_{S} K_{L} \rightarrow \pi^{+} \pi^{-} \pi^{+} \pi^{-}$. This latter option has been chosen by KLOE to place stringent limits on all three spatial components of $\Delta a_{\mu}$ [133, 90 :

$$
\begin{array}{r}
\Delta a_{X}=(-6.3 \pm 6.0) \times 10^{-18} \mathrm{GeV} \\
\Delta a_{Y}=(2.8 \pm 5.9) \times 10^{-18} \mathrm{GeV} \\
\Delta a_{Z}=(2.4 \pm 9.7) \times 10^{-18} \mathrm{GeV} .
\end{array}
$$

In fact the $\Delta a_{X, Y, Z}$ parameters can be evaluated performing a sidereal-time dependent analysis of the asymmetry:

$$
A(\Delta t)=\frac{N^{+}-N^{-}}{N^{+}+N^{-}},
$$

with $N^{+}=I\left(\pi^{+} \pi^{-}(+), \pi^{+} \pi^{-}(-) ; \Delta t>0\right)$ and $N^{-}=$ $I\left(\pi^{+} \pi^{-}(+), \pi^{+} \pi^{-}(-) ; \Delta t<0\right)$, where the two identical final states are distinguished by their emission in the forward $(\cos \theta>0)$ or backward $(\cos \theta<0)$ hemispheres (denoted by the symbols + and - , respectively), and $\Delta t$ is the time difference between $(+)$ and $(-) \pi^{+} \pi^{-}$decays.

In an asymmetric collider, quarkonium is produced with a sizable net momentum. In this case, all four components of $\Delta a_{\mu}$ for the neutral meson system contribute to observable effects. As a consequence, appropriate data binning would also allow up to four independent $C P T$ measurements. The existing asymmetric $B_{d}$ factories BaBar and Belle are able to perform such measurements. Results from the BaBar experiment bound various component combinations of $\Delta a_{\mu}$ for the $B_{d}$ meson to about
$10^{-14}$ to $10^{-15} \mathrm{GeV} 173$. The same study finds a $2.8 \sigma$ signal for sidereal variations 173 . While this significance level is still consistent with no effect, it clearly motivates further experimental $C P T$ - and Lorentz-violation studies with neutral meson systems.

To summarize this subsection, $C P T$ violation, which comes with Lorentz-symmetry breakdown in unitary field theories, is well motivated by various approaches to physics beyond the SM and GR. Experimental CPT and Lorentzsymmetry tests therefore are excellent tools in the search for new physics, possibly arising at the Planck scale. Such effects need not necessarily be correlated across various particles species, so measurements in different physical systems are typically inequivalent. In the quark sector, neutral meson interferometry is currently the only precision method for $C P T$-violation searches. While various experiments have placed tight constraints on $C P T$ breakdown in the $K, D$, and $B_{d}$ systems, only KLOE has been sensitive to all four $C P T$-violating SME coefficients, and KLOE-2 has the unique opportunity to perform a complete $C P T$ test in the kaon sector.

\subsection{Other $C P T$-symmetry tests at the $\phi$ factory}

Valuable information on $C P T$ invariance is provided by the study of $K_{S}$ semileptonic decays at the $\phi$-factory where large samples of tagged, pure, and monochromatic $K_{S}$ mesons can be isolated. In fact, when one kaon is detected at a time $t_{1} \gg \tau_{S}$, the decay amplitude of the entangledkaon state as in Eq.(17) factorizes and the system behaves as if the initial state was an incoherent mixture of states $\left|K_{S}\right\rangle_{\mathbf{k}} \otimes\left|K_{L}\right\rangle_{-\mathbf{k}}$ and $\left|K_{L}\right\rangle_{\mathbf{k}} \otimes\left|K_{S}\right\rangle_{-\mathbf{k}}$. Hence the detection of one kaon at large times tags one $\left|K_{S}\right\rangle$ state in the opposite direction. This is a unique feature of the $\phi$ factory exploited to select pure $K_{S}$ beams. At KLOE, the $K_{S}$ is tagged by identifying the interaction of the $K_{L}$ in the calorimeter. In fact about $50 \%$ of the $K_{L}$ 's reaches the calorimeter before decaying; their interactions $\left(K_{L^{-}}\right.$ -crash) are identified by a high-energy, neutral and delayed deposit in the calorimeter, i.e., not associated to any charged track in the event and delayed of $\sim 30 \mathrm{~ns}$ $\left(\beta_{K} \sim 0.22\right)$ with respect to photons coming from the interaction region (IP). In particular, $K_{S} \rightarrow \pi e \nu$ decays are selected requiring a $K_{L}$-crash and two tracks forming a vertex close to the IP and associated with two energy deposits in the calorimeter. Pions and electrons are identified using the time-of-flight technique. The number of signal events is normalized to the number of $K_{S} \rightarrow \pi^{+} \pi^{-}$ in the same data set.

The semileptonic $K_{S}$ decays are interesting for various reasons: the CKM matrix element $\left|V_{u s}\right|$ can be accurately extracted from the measurement of the semileptonic decay widths (Sect. 2). From the measurement of the lepton charge, the comparison between semileptonic asymmetry in $K_{S}$ decays, $A_{S}$, and the asymmetry $A_{L}$ in $K_{L}$ decays provides significant tests of both, the $\Delta S=\Delta Q$ rule, and $C P T$ invariance.

KLOE has obtained a very pure sample of $\simeq 13,000$ semileptonic $K_{S}$ decay events with an integrated lumi- 
nosity of $0.41 \mathrm{fb}^{-1}$ and measured for the first time the partial decay rates for transitions to final states of each lepton charge, $\Gamma\left(K_{S} \rightarrow e^{+} \pi^{-} \nu\right)$ and $\Gamma\left(K_{S} \rightarrow e^{-} \pi^{+} \bar{\nu}\right)$. The charge asymmetry

$$
\begin{aligned}
A_{S} & =\frac{\Gamma\left(K_{S} \rightarrow \pi^{-} e^{+} \nu\right)-\Gamma\left(K_{S} \rightarrow \pi^{+} e^{-} \bar{\nu}\right)}{\Gamma\left(K_{S} \rightarrow \pi^{-} e^{+} \nu\right)+\Gamma\left(K_{S} \rightarrow \pi^{+} e^{-} \bar{\nu}\right)} \\
& =\left(1.5 \pm 9.6_{\text {stat }} \pm 2.9_{\text {syst }}\right) \times 10^{-3} .
\end{aligned}
$$

has been obtained [54].

Assuming $C P T$ invariance, $A_{S}=A_{L}=2 \operatorname{Re} \epsilon \simeq 3 \times 10^{-3}$, where $\epsilon$ is the parameter related to $C P$ violation in $K \leftrightarrow \bar{K}$ $\Delta S=2$ transitions. The difference between the charge asymmetries,

$$
A_{S}-A_{L}=4\left(\operatorname{Re} \delta+\operatorname{Re} x_{-}\right),
$$

signals $C P T$ violation either in the mass matrix ( $\delta$ term), or in the decay amplitudes with $\Delta S \neq \Delta Q$ ( $x_{-}$term). The sum of the asymmetries,

$$
A_{S}+A_{L}=4(\operatorname{Re} \epsilon-\operatorname{Re} y),
$$

is related to $C P$ violation in the mass matrix ( $\epsilon$ term) and to $C P T$ violation in the $\Delta S=\Delta Q$ decay amplitude $(y$ term). Finally, the validity of the $\Delta S=\Delta Q$ rule in $C P T$ conserving transitions can be tested through the quantity:

$$
\operatorname{Re} x_{+}=\frac{1}{2} \frac{\Gamma\left(K_{S} \rightarrow \pi e \nu\right)-\Gamma\left(K_{L} \rightarrow \pi e \nu\right)}{\Gamma\left(K_{S} \rightarrow \pi e \nu\right)+\Gamma\left(K_{L} \rightarrow \pi e \nu\right)} .
$$

In the SM, Re $x_{+}$is of the order of $G_{F} m_{\pi}^{2} \sim 10^{-7}$, being due to second-order weak transitions.

Using the values of $A_{L}, \operatorname{Re} \delta$, and $\operatorname{Re} \epsilon$ from other experiments [18, KLOE obtained the following results [54]:

$$
\begin{aligned}
\operatorname{Re} y & =(0.4 \pm 2.5) \times 10^{-3} \\
\operatorname{Re} x_{-} & =(-0.8 \pm 2.5) \times 10^{-3} \\
\operatorname{Re} x_{+} & =(-1.2 \pm 3.6) \times 10^{-3}
\end{aligned}
$$

which are the most precise results on $C P T$ and $\Delta S=\Delta Q$ rule violation parameters in kaon semileptonic decays.

At KLOE-2, the integrated luminosity of $25 \mathrm{fb}^{-1}$ and better performance of the tracking system for decays close to the IP, beneficial for both the acceptance and the background rejection power, allow a total fractional uncertainty on the BR of $0.3 \%$ and thus sensitivity for obtaining evidence of a non-zero semileptonic asymmetry in $K_{S}$ decays.

The analogous measurements on $K_{S} \rightarrow \pi \mu \nu$ decays, never obtained before, are more difficult since poor $\pi-\mu$ separation power at low energy implies larger contamination on both, the semileptonic decay sample from $K_{S} \rightarrow$ $\pi^{+} \pi^{-}$, and the $K_{S} \rightarrow \pi^{-(+)} \mu^{+(-)} \nu$ sample from $K_{S} \rightarrow$ $\pi^{+(-)} \mu^{-(+)} \nu$.

A preliminary analysis of KLOE data has proven the capability to isolate the $K_{S} \rightarrow \pi \mu \nu$ events giving the first experimental evidence of the signal, and has shown the potentiality of reaching a BR precision better than $2 \%$ with a sample of $2.5 \mathrm{fb}^{-1}$. This translates to a precision of $\simeq 0.4 \%$ on the $\mathrm{BR}\left(K_{S} \rightarrow \pi \mu \nu\right)$ with KLOE-2.

\subsection{Quantitative Bohr's complementarity}

Bohr's complementarity principle or the closely related concept of duality in interferometric or double-slit like devices are at the heart of QM. The complementarity principle was phrased by Niels Bohr in an attempt to express the most fundamental difference between classical and quantum physics. According to this principle, and in sharp contrast to classical physics, in quantum physics we cannot capture all aspects of reality simultaneously, the information obtained in one single setup being always limited. By choosing the setup, e.g. the double-slit parameters, and thus the quantum state under investigation, the predictability, i.e., the a-priori knowledge of the path is simply calculated (particle-like information), whereas the interference pattern (wave-like information) is observed by the experiment. In the case of pure state, the sum of the squares of these two quantities adds up to one, i.e., available information, particle-like and wave-like, is conserved. This principle has been investigated both in theory and experiment mainly for photons, electrons and neutrons propagating through a double slit or through an interferometer. The neutral kaon system is optimal for testing Bohr's complementarity: it is an interfering system with no need of experimental devices for effecting interference and at an energy scale not usually tested from other systems. Moreover, the $C P$ violation makes these kaonic systems special. Via Bohr's complementarity relation new light can be shone upon $C P$ violation by showing that it moves information about reality to other aspects without violating the complementarity principle, i.e., from predictability $\mathcal{P}$ to coherence $\mathcal{V}$ and vice-versa, as discussed in Ref. [181.

The qualitative well-known statement that the observation of an interference pattern and the acquisition of which-way information are mutually exclusive has been rephrased to a quantitative statement [182,183]:

$$
\mathcal{P}^{2}(y)+\mathcal{V}_{0}^{2}(y) \leq 1,
$$

where the equality is valid for pure quantum states and the inequality for mixed ones. $\mathcal{V}_{0}(y)$ is the fringe visibility which quantifies the sharpness or contrast of the interference pattern (the wave-like property) and can depend on an external parameter $y$, whereas $\mathcal{P}(y)$ denotes the path predictability, i.e., the a-priori knowledge one can have on the path taken by the interfering system (the particle-like property). It is defined by

$$
\mathcal{P}(y)=\left|p_{I}(y)-p_{I I}(y)\right|
$$

where $p_{I}(y)$ and $p_{I I}(y)$ are the probabilities for taking each path $\left(p_{I}(y)+p_{I I}(y)=1\right)$. The predictability and visibility are in general dependent from external parameters. For example, in the usual double-slit experiment the intensity is given by

$$
I(y)=F(y)\left(1+\mathcal{V}_{0}(y) \cos (\phi(y))\right),
$$

where $F(y)$ is specific for each setup and $\phi(y)$ is the phasedifference between the two paths. The variable $y$ characterizes in this case the detector position, thus visibility 
and predictability are $y$-dependent. In Ref. 184 physical situations were investigated for which the expressions of $\mathcal{V}_{0}(y), \mathcal{P}(y)$ and $\phi(y)$ can be calculated analytically. These systems include interference patterns of various types of double-slit experiments ( $y$ is linked to position), the oscillations due to particle mixing ( $y$ is linked to time) as in case of kaon systems, and also Mott scattering experiments on identical particles or nuclei ( $y$ is linked to a scattering angle). All these two-state systems can be treated in a unified way via the generalized complementarity relation. Here we investigate shortly the neutral kaon case while the reader can refer to Ref. [184] for more details and discussion on other systems.

The time evolution of an initial $K^{0}$ state can be described by (in the following $C P$ violation effects can safely be neglected)

$$
\left|K^{0}(t)\right\rangle \cong \frac{e^{-\frac{\Delta \Gamma}{4} t}}{\sqrt{2 \cosh \left(\frac{\Delta \Gamma}{2} t\right)}}\left\{e^{i \Delta m \cdot t+\frac{\Delta \Gamma}{2} t}\left|K_{S}\right\rangle+\left|K_{L}\right\rangle\right\}
$$

where $\Delta \Gamma=\Gamma_{L}-\Gamma_{S}<0$. The state of Eq. (41) can be interpreted as follows. The two mass eigenstates $\left|K_{S}\right\rangle,\left|K_{L}\right\rangle$ represent the two slits. At time $t=0$ both slits have the same width, as time evolves one slit decreases as compared to the other, however, in addition the whole double slit shrinks due to the decay property of the kaons. This analogy gets more obvious if we consider for an initial $K^{0}$ the probabilities for finding a $K^{0}$ or a $\bar{K}^{0}$ state at time $t$, i.e., the strangeness oscillation:

$$
\begin{aligned}
& P\left(K^{0}, t\right)=\left|\left\langle K^{0} \mid K^{0}(t)\right\rangle\right|^{2}=\frac{1}{2}\left\{1+\frac{\cos (\Delta m \cdot t)}{\cosh \left(\frac{\Delta \Gamma}{2} t\right)}\right\} \\
& P\left(\bar{K}^{0}, t\right)=\left|\left\langle\bar{K}^{0} \mid K^{0}(t)\right\rangle\right|^{2}=\frac{1}{2}\left\{1-\frac{\cos (\Delta m \cdot t)}{\cosh \left(\frac{\Delta \Gamma}{2} t\right)}\right\}
\end{aligned}
$$

We observe that the oscillating phase is given by $\phi(t)=$ $\Delta m \cdot t$ and the time dependent visibility by

$$
\mathcal{V}_{0}(t)=\frac{1}{\cosh \left(\frac{\Delta \Gamma}{2} t\right)}
$$

maximal at $t=0$. The "which width" information, analogously to the "which way" information in usual double-slit experiments, can be directly calculated from Eq. (41) and gives the following predictability,

$$
\begin{aligned}
\mathcal{P}(t) & =\left|P\left(K_{S}, t\right)-P\left(K_{L}, t\right)\right|=\left|\frac{e^{\frac{\Delta \Gamma}{2} t}-e^{-\frac{\Delta \Gamma}{2} t}}{2 \cosh \left(\frac{\Delta \Gamma}{2} t\right)}\right| \\
& =\left|\tanh \left(\frac{\Delta \Gamma}{2} t\right)\right| .
\end{aligned}
$$

The larger the time $t$ is, the more probable is the propagation of the $K_{L}$ component, because the $K_{S}$ component has died out, the predictability converges to its upper bound 1. Both expressions, the predictability (44) and the visibility (43), satisfy the complementary relation (38) for all times $t$ :

$$
\mathcal{P}^{2}(t)+\mathcal{V}_{0}^{2}(t)=\tanh ^{2}\left(\frac{\Delta \Gamma}{2} t\right)+\frac{1}{\cosh ^{2}\left(\frac{\Delta \Gamma}{2} t\right)}=1
$$

For time $t=0$ there is full interference, the visibility is $\mathcal{V}_{0}(t=0)=1$, and we have no information about the lifetimes or widths, $\mathcal{P}(t=0)=0$. This corresponds to the usual double-slit scenario. For large times, i.e. $t \gg$ $1 / \Gamma_{S}$, the kaon is most probable in a long-lived state $K_{L}$ and no interference is observed, we have information on the "which width". For times between the two extremes we obtain partially information on "which width" and on the interference contrast due to the natural instability of the kaons. For pure systems however the information in Eq. (38) or Eq. (45) is always maximal.

This strict formal analogy between the neutral kaon system and the double-slit device can be fully appreciated when exploited to implement a quantum eraser experiment, which could be feasible at KLOE-2, as briefly explained in the next subsection.

\subsubsection{The kaonic quantum eraser}

Photons intereference was demonstrated two hundred years ago by Thomas Young. There is no physical reason why even heavier particles should not interfere, and also experiments with very massive "particles" like the fullerenes have proven this fundamental feature of QM. Further studies have shown that the knowledge of the path to the double slit is the reason why interference is lost. The gedanken experiment of Scully and Drühl in 1982 [185] surprised the physics community demonstrating that if the knowledge of the particle path is erased, the interference is brought back again.

Since that work many different types of quantum erasures have been analyzed and experiments were performed with atom interferometers and entangled photons where the quantum erasure in the so-called delayed-choice mode captures better the essence and the most subtle aspects of the eraser phenomenon. In this case the meter, the quantum system which carries the mark on the path, is a system spatially separated from the interfering system, referred to as object system. The decision to erase or not the mark of the meter system - and therefore to observe or not the interference - can be taken long after the measurement on the object system has been completed. This was nicely phrased by Aharonov and Zubairy in their Science review "Time and the Quantum: Erasing the Past and Impacting the Future" [186] where also the kaon eraser proposed in Refs. 187,188 is presented.

The quantum erasure experiments were mostly based on some kind of double-slit device or on entangled states. which are both available at DA $\Phi$ NE .

All existing experiments with usual quantum systems as photons, based on entanglement and double-slit devices, can be divided into two classes. In the first, the erasure of the "which way" information is fully-actively performed by an experimenter, i.e., choosing the appropriate experimental setup. In the second class erasure is 
obtained by a probabilistic process, e.g. a beam splitter. The erasure of the "which way" information is in this case partially active. Both options of erasure can be tested with the neutral kaon system. In addition, neutral kaons can be measured by a different, completely passive procedure. Herewith we obtain four different erasure options, two more than with usual quantum systems. An example of active measurement is placing a matter block into the beam for the determination of kaon strangeness from final-state particle identification. It is active because the experimenter chooses to measure the strangeness of the kaons during their time evolution and also the time when the measurement is performed. But there is as well the option of simply waiting for the kaon decay. If by chance a semileptonic decay is detected then the kaon strangeness is determined as well, without the experimenter's control on the mode and the decay time, with a passive-measurement procedure.

In summary, these two plus two possibilities of quantum erasure prove in a new way the very concept of a quantum eraser. The proposed quantum-eraser procedures give the same probabilities even regardless of the temporal ordering of the measurements. Thus kaonic erasers can also be operated in the so called delayed-choice mode as discussed in Refs. [189, 190, shedding new light on the very nature of the quantum eraser and contributing to clarify the eraser working principle hot-debated in literature.

\subsection{KLOE-2 prospects on kaon interferometry}

The decay mode $\phi \rightarrow K_{S} K_{L} \rightarrow \pi^{+} \pi^{-} \pi^{+} \pi^{-}$is very rich in physics. In general all decoherence effects including $C P T-$ violation related to decoherence phenomena should manifest as a deviation from the QM prediction $I\left(\pi^{+} \pi^{-}, \pi^{+} \pi^{-}\right.$; $\Delta t=0)=0$. Hence the reconstruction of events in the region $\Delta t \approx 0$, i.e., with vertices close to the IP, is crucial for precise determination of the parameters related to the $C P T$ violation and to the decoherence. The vertex resolution affects the $I\left(\pi^{+} \pi^{-}, \pi^{+} \pi^{-} ; \Delta t\right)$ distribution precisely in that region as shown in Fig. 6, and impacts the measurement of the decoherence parameter in two ways: i) reducing the sensitivity of the fit with respect to the accuracy expected from statistical fluctuations only; ii) introducing a source of systematic uncertainty. The major upgrade planned at KLOE-2 is the addition of the vertex detector between the spherical beam pipe and the drift chamber as presented in Sect. 1, designed to reach an experimental resolution on charged vertices of $\sigma_{\Delta t} \approx 0.3 \tau_{S}$. In Fig. 7 the statistical uncertainty on several decoherence and $C P T$-violating parameters is shown as a function of the integrated luminosity for the case $\sigma_{\Delta t} \approx \tau_{S}$ (KLOE resolution), and for $\sigma_{\Delta t} \approx 0.3 \tau_{S}$ (KLOE-2 resolution with the inner tracker). The improvement on vertex resolution leads to an increase of the experimental sensitivity by a factor of two. The physics program concerning interferometry is summarized in Tab. 7 where the KLOE2 sensitivity to the main parameters that can be extracted from $I\left(f_{1}, f_{2} ; \Delta t\right)$ with an integrated luminosity of $25 \mathrm{fb}^{-1}$

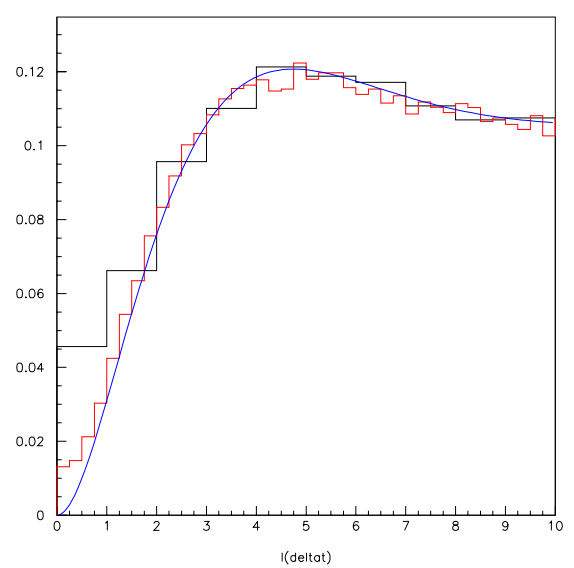

Fig. 6: Monte Carlo simulation of the $I\left(\pi^{+} \pi^{-}, \pi^{+} \pi^{-} ; \Delta t\right)$ as a function of $|\Delta t|$ (in $\tau_{S}$ units) with the present KLOE resolution $\sigma_{\Delta t} \approx \tau_{S}$ (histogram with large bins), with an improved resolution $\sigma_{\Delta t} \approx 0.3 \tau_{S}$ (histogram with small bins), and in the ideal case (solid line).
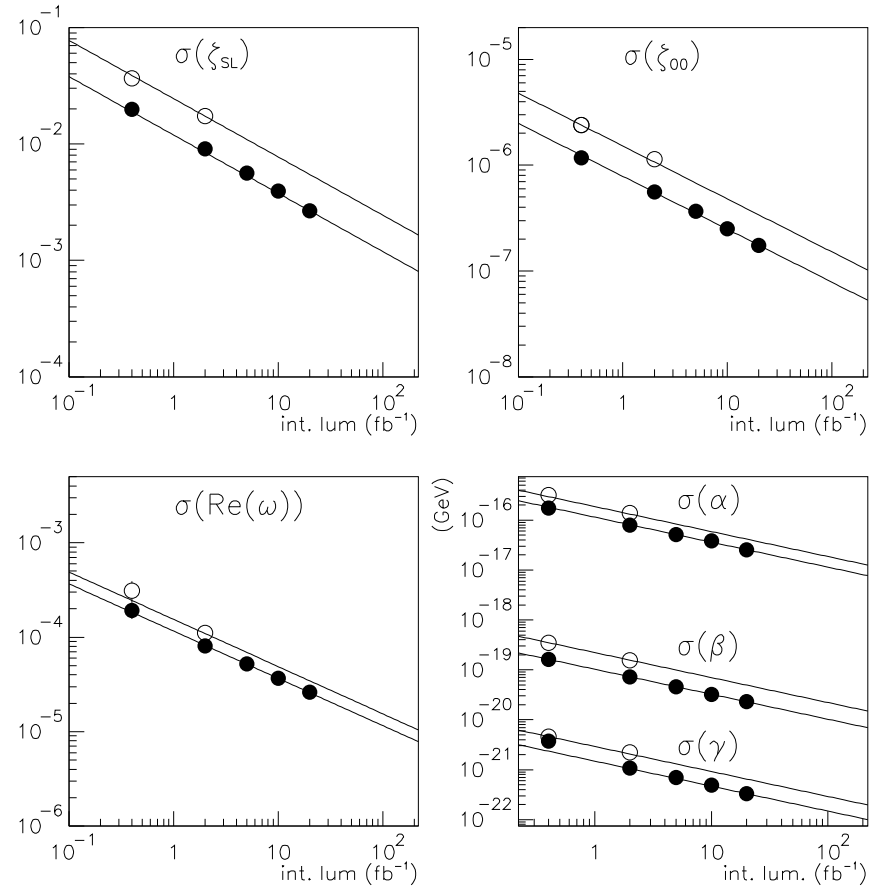

Fig. 7: The sensitivity to the $\zeta_{S L}, \zeta_{0 \overline{0}}$, Re $\omega$ and $\alpha, \beta, \gamma$ parameters with the present KLOE resolution $\sigma_{\Delta t} \approx \tau_{S}$ (open circles), and with the improved resolution $\sigma_{\Delta t} \approx$ $0.3 \tau_{S}$ expected with the IT at KLOE-2 (full circles).

are listed and compared with the best published measurements. Although not exhaustive, the list is nevertheless indicative of the extent to which the experimental results at KLOE-2 can contribute to this field. 
Table 7: KLOE-2 sensitivity on several parameters.

\begin{tabular}{|c|c|c|c|c|}
\hline$\overline{f_{1}}$ & $\mathbf{f}_{2}$ & Parameter & Present best measurement & KLOE-2 $\left(25 \mathrm{fb}^{-1}\right)$ \\
\hline$K_{S} \rightarrow \pi e \nu$ & & $\overline{A_{S}}$ & $(1.5 \pm 11) \times 10^{-3}$ & $\pm 1 \times 10^{-3}$ \\
\hline$\pi^{+} \pi^{-}$ & $\pi l \nu$ & $\overline{A_{L}}$ & $(332.2 \pm 5.8 \pm 4.7) \times 10^{-5}$ & $\pm 4 \times 10^{-5}$ \\
\hline$\pi^{+} \pi^{-}$ & $\pi^{0} \pi^{0}$ & $\operatorname{Re} \frac{\epsilon^{\prime}}{\epsilon}$ & $(1.65 \pm 0.26) \times 10^{-3}(\mathrm{PDG})$ & $\pm 0.3 \times 10^{-3}$ \\
\hline$\pi^{+} \pi^{-}$ & $\pi^{0} \pi^{0}$ & $\operatorname{Im} \frac{\epsilon^{\prime}}{\epsilon}$ & $(-1.2 \pm 2.3) \times 10^{-3}(\mathrm{PDG})$ & $\pm 4 \times 10^{-3}$ \\
\hline$\pi^{+} l^{-} \bar{\nu}$ & $\pi^{-} l^{+} \nu$ & $\left(\operatorname{Re} \delta+\operatorname{Re} x_{-}\right)$ & $\begin{array}{c}\operatorname{Re} \delta=(0.25 \pm 0.23) \times 10^{-3}(\mathrm{PDG}) \\
\operatorname{Re} x_{-}=(-4.2 \pm 1.7) \times 10^{-3}(\mathrm{PDG})\end{array}$ & $\pm 0.3 \times 10^{-3}$ \\
\hline$\pi^{+} l^{-} \bar{\nu}$ & $\pi^{-} l^{+} \nu$ & $\left(\operatorname{Im} \delta+\operatorname{Im} x_{+}\right)$ & $\begin{array}{l}\operatorname{Im} \delta=(-0.6 \pm 1.9) \times 10^{-5}(\mathrm{PDG}) \\
\operatorname{Im} x_{+}=(0.2 \pm 2.2) \times 10^{-3}(\mathrm{PDG})\end{array}$ & $\pm 4 \times 10^{-3}$ \\
\hline$\pi^{+} \pi$ & $\pi^{+} \pi^{-}$ & $\Delta m$ & $5.288 \pm 0.043 \times 10^{9} \mathrm{~s}^{-1}$ & $\pm 0.05 \times 10^{9} \mathrm{~s}^{-1}$ \\
\hline$\pi^{+} \pi^{-}$ & $\pi^{+} \pi^{-}$ & $\zeta_{S L}$ & $(0.3 \pm 1.9) \times 10^{-2}$ & $\pm 0.2 \times 10^{-2}$ \\
\hline$\pi^{+} \pi^{-}$ & $\pi^{+} \pi^{-}$ & $\zeta_{0 \overline{0}}$ & $(0.1 \pm 1.0) \times 10^{-6}$ & $\pm 0.1 \times 10^{-6}$ \\
\hline$\pi^{+} \pi^{-}$ & $\pi^{+} \pi^{-}$ & $\alpha$ & $(-0.5 \pm 2.8) \times 10^{-17} \mathrm{GeV}$ & $\pm 2 \times 10^{-17} \mathrm{GeV}$ \\
\hline$\pi^{+} \pi^{-}$ & $\pi^{+} \pi^{-}$ & $\beta$ & $(2.5 \pm 2.3) \times 10^{-19} \mathrm{GeV}$ & $\pm 0.2 \times 10^{-19} \mathrm{GeV}$ \\
\hline$\pi^{+} \pi^{-}$ & $\pi^{+} \pi^{-}$ & $\gamma$ & $\begin{array}{l}(1.1 \pm 2.5) \times 10^{-21} \mathrm{GeV} \\
(\text { compl. pos. hyp. }) \\
(0.7 \pm 1.2) \times 10^{-21} \mathrm{GeV}\end{array}$ & $\begin{array}{l} \pm 0.3 \times 10^{-21} \mathrm{GeV} \\
\pm 0.2 \times 10^{-21} \mathrm{GeV}\end{array}$ \\
\hline$\pi^{+} \pi^{-}$ & $\pi^{+} \pi^{-}$ & Rew & $\left(-1.6_{-2.1}^{+3.0} \pm 0.4\right) \times 10^{-4}$ & $\pm 3 \times 10^{-5}$ \\
\hline$\pi^{+} \pi^{-}$ & $\pi^{+} \pi^{-}$ & $\operatorname{Im} \omega$ & $\left(-1.7_{-3.0}^{+3.3} \pm 1.2\right) \times 10^{-4}$ & $\pm 4 \times 10^{-5}$ \\
\hline$K_{S, L} \rightarrow \pi e \nu$ & & $\Delta a_{0}$ & (prelim.: $\left.(0.4 \pm 1.8) \times 10^{-17} \mathrm{GeV}\right)$ & $\pm 2 \times 10^{-18} \mathrm{GeV}$ \\
\hline$\pi^{+} \pi^{-}$ & $\pi^{+} \pi^{-}$ & $\Delta a_{Z}$ & (prelim.: $\left.(2.4 \pm 9.7) \times 10^{-18} \mathrm{GeV}\right)$ & $\pm 1 \times 10^{-18} \mathrm{GeV}$ \\
\hline$\pi^{+} \pi^{-}$ & $\pi^{+} \pi^{-}$ & $\Delta a_{X}, \Delta a_{Y}$ & (prelim.: $\left.<9.2 \times 10^{-22} \mathrm{GeV}\right)$ & $\pm 6 \times 10^{-19} \mathrm{GeV}$ \\
\hline
\end{tabular}

\section{Low Energy QCD}

The interest for hadronic physics at low energy is not only related to the development of the EFT describing to some extent the non-perturbative phenomena, but also to precision physics involving light flavors $\mathrm{u}, \mathrm{d}, \mathrm{s}$, where the sensitivity for testing the SM is often limited by the knowledge of hadronic interactions (strong and electroweak).

The impact on the field of the measurements of semileptonic and leptonic kaon channels has already been discussed in Sect. 2 .

ChPT is the theoretical framework to study low-energy implications of the symmetry properties of the QCD theory 191,192. The pseudoscalar octet $(\pi, \mathrm{K}, \eta)$ is identified as the multiplet of massless Goldstone bosons associated to the chiral-symmetry breaking and the scalars are used as degrees of freedom of the effective field theory at energy scale $\lambda<\mathrm{M}_{\rho}$.

This section is mostly dedicated to the measurements of non-leptonic and radiative decays of the $K_{S}$ and the $\eta$ meson; other significant results in the field can be achieved at KLOE-2 with the study of events in the continuum, reviewed in Sects. 56 .

\subsection{Rare kaon decays}

The measurement of $K_{S}$ decays at the $\phi$-factory has the unique feature to rely on pure $K_{S}$ beams, tagged by the reconstruction of $K_{L}$ decays and $K_{L}$ interactions in the calorimeter. Background sources are limited to the dominant $K_{S}$ decay channels, $K_{S} \rightarrow \pi \pi$, severely constrained by the $K_{L} 4$-momentum reconstruction.
With a target integrated luminosity of $25 \mathrm{fb}^{-1}$, accessible channels include non-leptonic and radiative decays with $\mathrm{BR}$ down to $\mathcal{O}\left(10^{-9}\right), K_{S} \rightarrow 3 \pi, K_{S} \rightarrow \gamma \gamma, K_{S} \rightarrow$ $\pi^{0} \gamma \gamma, K_{S} \rightarrow \pi^{0} \ell^{+} \ell^{-}, K_{S} \rightarrow \pi^{+} \pi^{-} \gamma, K_{S} \rightarrow \pi^{+} \pi^{-} e^{+} e^{-}$.

We can divide kaon decays into three categories: i) long-distance dominated (LD), ii) with comparable shortand long-distance contributions and iii) short-distance dominated (SD) decays. Categories i) and ii) are those of interest for KLOE-2: the LD modes are addressed by the $K_{S}$-decay analysis. They are properly described by ChPT 193, 194, 195, 196, 197, whose $\Delta S=1$ lagrangian at $\mathcal{O}\left(p^{4}\right)$ reads:

$$
\mathcal{L}_{\Delta S=1}=G_{8} F^{4} \underbrace{\left\langle\lambda_{6} D_{\mu} U^{\dagger} D^{\mu} U\right\rangle}_{K \rightarrow \pi \pi / 3 \pi, \gamma \gamma}+\underbrace{G_{8} F^{2} \sum N_{i} W_{i}}_{K^{+} \rightarrow \pi^{+} \gamma \gamma, K \rightarrow \pi \pi \gamma}+\ldots
$$

$G_{8}$ is fixed by the $K \rightarrow \pi \pi$ amplitudes; the first $\left(\mathcal{L}_{\Delta S=1}^{2}\right)$ and the second term $\left(\mathcal{L}_{\Delta S=1}^{4}\right)$ represent the $\mathcal{O}\left(p^{2}\right)$ and $\mathcal{O}\left(p^{4}\right)$ weak lagrangian, respectively [198, 199. There are 37 counterterm coefficients, $N_{i}$ 's, and operators, $W_{i}$ 's. Unfortunately the low energy constants $N_{i}$ 's (LECs) are both theoretically and phenomenologically very poorly known. New measurements of the non-leptonic and radiative decays are relevant to test the weak ChPT Lagrangian and help settling the LECs, which encode the underlying quark dynamics.

The Vector Dominance Model, by analogy to the treatment of the strong amplitudes, has been used to determine the relevant combination of LECs in processes with vector-meson-exchange contributions. As an example, the results for radiative non-leptonic kaon decays are shown in Tab. 8 198, 199 . 
Table 8: Combinations of the counterterm coefficients in radiative non-leptonic kaon decays.

\begin{tabular}{l|l} 
Decay & $\mathcal{L}_{\Delta S=1}^{4}$ \\
\hline$K^{+} \rightarrow \pi^{+} l^{+} l^{-}$ & $N_{14}^{r}-N_{15}^{r}$ \\
$K_{S} \rightarrow \pi^{0} l^{+} l^{-}$ & $2 N_{14}^{r}+N_{15}^{r}$ \\
$K^{ \pm} \rightarrow \pi^{ \pm} \gamma \gamma$ & $N_{14}-N_{15}-2 N_{18}$ \\
$K_{S} \rightarrow \pi^{+} \pi^{-} \gamma$ & $N_{14}-N_{15}-N_{16}-N_{17}$ \\
$K^{ \pm} \rightarrow \pi^{ \pm} \pi^{0} \gamma$ & $N_{14}-N_{15}-N_{16}-N_{17}$ \\
$K_{L} \rightarrow \pi^{+} \pi^{-} e^{+} e^{-}$ & $N_{14}^{r}+2 N_{15}^{r}-3\left(N_{16}^{r}-N_{17}\right)$ \\
$K^{+} \rightarrow \pi^{+} \pi^{0} e^{+} e^{-}$ & $N_{14}^{r}+2 N_{15}^{r}-3\left(N_{16}^{r}-N_{17}\right)$ \\
$K_{S} \rightarrow \pi^{+} \pi^{-} e^{+} e^{-}$ & $N_{14}^{r}-N_{15}^{r}-3\left(N_{16}^{r}+N_{17}\right)$
\end{tabular}

\subsection{1 $K \rightarrow 3 \pi$}

The amplitudes of $K \rightarrow 3 \pi$ decays can be approximated by a second-order polynomial in the pion kinetic energy $\mathrm{T}_{i}$ using the $\mathrm{X}, \mathrm{Y}$ variables 2 . An alternative parametrization has been recently proposed [200,18] to evaluate how the Dalitz plot is affected by $\pi \pi$ re-scattering and in particular to extract the $\pi \pi$ scattering lenghts from the analysis of the cusp in $K^{+} \rightarrow \pi^{+} \pi^{0} \pi^{0}$. Chiral expansion relates $K \rightarrow$ $\pi \pi$ to $K \rightarrow 3 \pi$ transitions so that significant constraints on the values of the $\mathcal{O}\left(p^{4}\right)$ counterterms of Eq.(46) have been obtained from the Dalitz plot of the experimental data, largely dominated by $\Delta I=1 / 2$ transitions.

Lack of data on the subleading $\Delta I=3 / 2$ amplitudes calls for the measurement of the $K_{S} \rightarrow \pi^{+} \pi^{-} \pi^{0}$ decay at KLOE-2. In fact, the $K_{S} \rightarrow \pi^{+} \pi^{-} \pi^{0}$ amplitude:

$$
A\left(K_{S} \rightarrow \pi^{+} \pi^{-} \pi^{0}\right)=\frac{2}{3} b_{2} X-\frac{4}{3} d_{2} X Y,
$$

is directly related to the $\Delta I=3 / 2, L=1$ transitions, being the $\mathrm{I}=0$ isospin final state suppressed by the high angular momentum barrier 201.

A preliminary but rather detailed search for this decay has been performed at KLOE with $740 \mathrm{pb}^{-1}$ of integrated luminosity. Main backgrounds come from charged kaon events in which one $\pi^{0}$ mimics the $K_{L}$ interaction in the calorimeter used to tag the $K_{S}$ decay, and from $K_{S} \rightarrow \pi^{0} \pi^{0}$ events with one $\pi^{0} \rightarrow e^{+} e^{-} \gamma$. Kinematic constraints are effective for background suppression. Final contaminations at the level of $20-30 \%$ have been obtained selecting the signal sample with a global efficiency of $1-2 \%$. KLOE-2 can eventually select from 50 to few hundreds events, depending on the total integrated luminosity and also on the fraction of data collected after the installation of the detector upgrades, all of them beneficial for improving the acceptance and the reconstruction capability of these events.

The $C P$-violating transition $K_{S} \rightarrow \pi^{0} \pi^{0} \pi^{0}$ receives contributions from $\mathrm{I}=1$ final state only. KLOE has obtained the upper limit $\operatorname{BR}\left(K_{S} \rightarrow 3 \pi^{0}\right) \leq 1.2 \times 10^{-7}$ at $90 \%$ C.L., using a sample of $450 \mathrm{pb}^{-1}$ [65].

\footnotetext{
2 Neglecting the pion mass difference, $\mathrm{X}=\frac{2 m_{k}}{m_{\pi}^{2}}\left(\mathrm{~T}_{2}-\mathrm{T}_{1}\right)$; $\mathrm{Y}=\frac{2 m_{k}}{m_{\pi}^{2}}\left(\mathrm{~T}_{3}+m_{\pi}-\frac{1}{3} m_{k}\right)$
}

The main background source is given by incorrectly reconstructed $K_{S} \rightarrow \pi^{0} \pi^{0}$ decays, when the photon-energy deposit in the calorimeter is reconstructed as split clusters, or additional photons from machine background are assigned to the $K_{S}$ decay. The kinematic constraints applied to fully reconstructed final states have allowed KLOE to improve by a factor of ten the upper limit on this channel and to increase the sensitivity on the $C P$ and $C P T$ tests using the unitary condition (Bell-Steinberger relation) as explained in Ref. 202. KLOE, with a $K_{S}$ tagging efficiency of about $23 \%$ and a selection efficiency on the signal of $\sim 25 \%$ has found 2 events to be compared with 3.13 expected by Monte Carlo simulation of the background sources. Since then, further improvements on the clustering procedure to recover erroneously-split photon clusters are proven to reduce contamination by a factor of six while leaving the signal efficiency unaffected. There are currently studies underway to evaluate the increase in acceptance from the installation of the crystal calorimeters (cf. Sec1), and in the $K_{S}$ tagging efficiency resulting from the addition of the $K_{L} \rightarrow \pi^{+} \pi^{-} \pi^{0}$ sample to the events with $K_{L}$ interactions in the calorimeter used in the KLOE analysis. In any case, as a conservative estimate, KLOE-2 can obtain an upper limit lower than $10^{-8}$ with one year of data taking.

\subsection{2 $K_{S} \rightarrow \gamma^{(*)} \gamma^{(*)} / K_{S} \rightarrow \pi^{0} \gamma \gamma$}

$K_{S} \rightarrow \gamma \gamma$ does not receive any SD contribution while LD terms starts at $\mathcal{O}\left(p^{4}\right)$ without counterterm structure. This implies that: i) we have only one loop contribution and ii) this contribution is scale-independent 203,204]. The $B R\left(K_{S} \rightarrow \gamma \gamma\right)=2.1 \times 10^{-6}$ is predicted at $\mathcal{O}\left(p^{4}\right)$ in terms of two known LECs of lowest order. From a naive dimensional analysis higher order contributions are expected to be suppressed by a factor $\mathrm{m}_{k}^{2} /\left(4 \pi \mathrm{F}_{\pi}\right)^{2} \sim 0.2$. This process is the ideal test of the ChPT (and in general of the EFT) at the quantum level. At present, for the branching fraction, we have:

$$
\begin{aligned}
& \mathrm{ChPT} \text { at } \mathcal{O}\left(p^{4}\right) \quad 2.1 \times 10^{-6} \\
& \mathrm{NA} 48 \quad(2.713 \pm 0.063) \times 10^{-6} \\
& \mathrm{KLOE} \quad(2.26 \pm 0.13) \times 10^{-6}
\end{aligned}
$$

with the recent measurements differing by $\sim 3 \sigma$ 's 205,64 , as also shown in Fig. 8 . These results are based on different experimental methods. At the hadronic machines, the branching ratio has been obtained from the simultaneous measurement of $K_{L}$ and $K_{S}$ decays to the same final state, $K_{S, L} \rightarrow \gamma \gamma$. The subtraction of the $K_{L}$ background has been performed on the basis of precision measurements of the ratio $B R\left(K_{L} \rightarrow \gamma \gamma\right) / B R\left(K_{L} \rightarrow 3 \pi^{0}\right)$. At the $\phi$ factory, the $K_{S}$ decays are tagged by $K_{L}$ interactions in the calorimeter and the $K_{S} \rightarrow \gamma \gamma$ signal has to be separated from the $K_{S} \rightarrow \pi^{0} \pi^{0}$ decays with lost or unresolved photon clusters.

The KLOE result is fully compatible with $\mathcal{O}\left(p^{4}\right)$ calculations in ChPT 203] thus severely constraining $\mathcal{O}\left(p^{6}\right)$ 


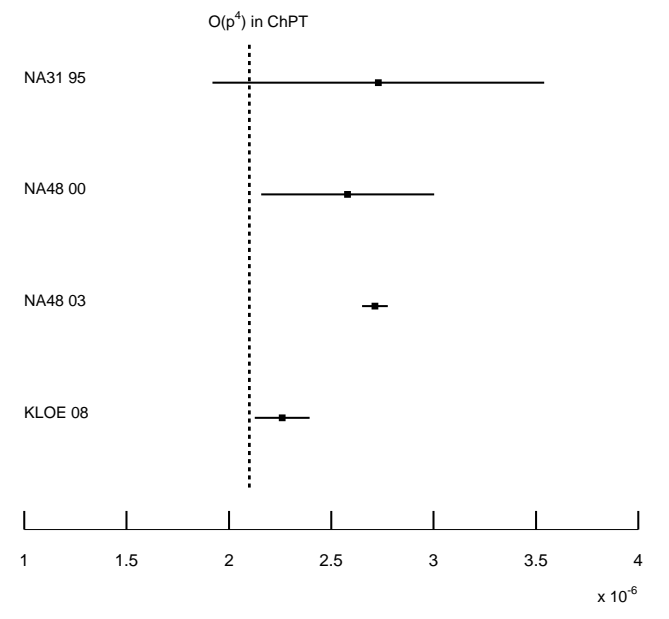

Fig. 8: $\operatorname{BR}\left(K_{S} \rightarrow \gamma \gamma\right)$ at $\mathcal{O}\left(p^{4}\right)$ in $\mathrm{ChPT}$ (dashed line) compared with the experimental results. It should be considered that from a naive dimensional analysis higher order theoretical contributions are expected to be suppressed by a factor $\mathrm{m}_{k}^{2} /\left(4 \pi \mathrm{F}_{\pi}\right)^{2} \sim 0.2$.

contributions to this process, while the NA48 measurement points to sizable higher-order corrections, $\frac{A\left(\mathcal{O}\left(p^{6}\right)\right)}{A\left(\mathcal{O}\left(p^{4}\right)\right)} \sim$ $15 \%$. KLOE-2 will improve both sample size and data quality, the latter thanks to the detector upgrade, namely the crystal calorimeters CCALT important for the identification of $K_{S} \rightarrow \pi^{0} \pi^{0}$ with photons at low angle with respect to the beam direction.

The KLOE-2 measurement can clarify the disagreement between KLOE and NA48 205,64 and help settling the $\mathcal{O}\left(p^{6}\right)$ contributions to $A\left(K_{L} \rightarrow \pi^{0} \gamma \gamma\right)$, related by chiral symmetry only to the $K_{S} \rightarrow \gamma \gamma$ terms [206. The $K_{S} \rightarrow \pi^{0} \gamma \gamma$ decay is dominated by pole contributions, from $\pi^{0}$ and $\eta: K_{S} \rightarrow \pi^{0} " \pi^{0 "}$ " " $\left.\eta "\right) \rightarrow \pi^{0} \gamma \gamma$. The diphoton invariant mass spectrum should be better assessed for a significant test of the pole dominance and for the study of the weak chiral vertex, $K_{S} \rightarrow \pi^{0}$ " $P$ ".

The NA48 analysis of the $K_{S} \rightarrow \pi^{0} \gamma \gamma$ has provided the branching fraction and the diphoton invariant-mass $\left(m_{\gamma \gamma}\right)$ spectrum [207] at high $z\left(z=m_{\gamma \gamma}^{2} / m_{K}^{2}\right)$. The result is based on 31 candidates, with an expected background of $13.7 \pm 3.2$ events:

$$
B R\left(K_{S} \rightarrow \pi^{0} \gamma \gamma\right)_{z>0.2}=(4.9 \pm 1.6 \pm 0.9) \times 10^{-8}
$$

to be compared with the theoretical prediction of $3.8 \times$ $10^{-8} 208$.

At KLOE-2 the acceptance for $K_{S} \rightarrow \pi^{0} \gamma \gamma$ is about $60 \%$, as for $K_{S} \rightarrow \pi^{0} \pi^{0}$, and we expect 10 events $/ \mathrm{fb}^{-1}$ to be sorted out from the dominant $K_{S} \rightarrow \pi^{0} \pi^{0}$ sample.

The $K_{S}$ decays into lepton pairs $\left(e^{+} e^{-}, \mu^{+} \mu^{-}\right)$are due to $\Delta S=1$ flavor-changing neutral-current (FCNC) transitions. Decay amplitudes receive contributions both from LD effects, dominated by the $\gamma \gamma$ intermediate state shown in Fig. 9, and from SD interactions, due to box and penguin diagrams via $W, Z$ exchange. The SD contribu-

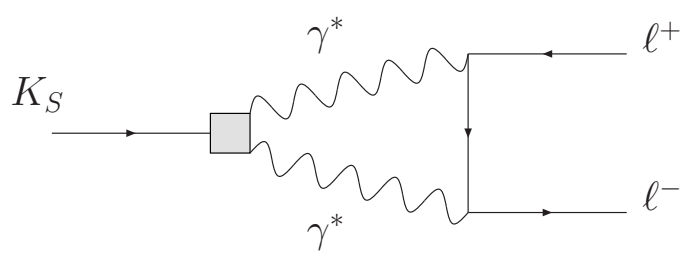

Fig. 9: Long distance contribution to $K_{S} \rightarrow \ell^{+} \ell^{-}$process mediated by two-photon exchange.

tion can be rather precisely evaluated in the SM, while the $\mathrm{LD}$ terms can be determined in ChPT. At lowest order, $\mathcal{O}\left(p^{4}\right)$, one obtains [209]:

$$
\begin{aligned}
\Gamma\left(K_{S} \rightarrow \mu^{+} \mu^{-}\right) / \Gamma\left(K_{S} \rightarrow \gamma \gamma\right) & \simeq 2 \times 10^{-6}, \\
\Gamma\left(K_{S} \rightarrow e^{+} e^{-}\right) / \Gamma\left(K_{S} \rightarrow \gamma \gamma\right) & \simeq 8 \times 10^{-9}
\end{aligned}
$$

which translate into $\operatorname{BR}\left(K_{S} \rightarrow \mu^{+} \mu^{-}\right) \simeq 5 \times 10^{-12}$ and $\operatorname{BR}\left(K_{S} \rightarrow e^{+} e^{-}\right) \simeq 2 \times 10^{-14}$. Significantly higher values would point to new physics (NP).

Using the complete data set KLOE has obtained an upper limit on the electron channel, $B R\left(K_{S} \rightarrow e^{+} e^{-}\right) \leq$ $9 \times 10^{-9} 66$, one order of magnitude more stringent than previous experimental results 210. KLOE-2 can improve the sensitivity for both the leptonic channels to the $10^{-9}$ level.

\subsection{3 $K_{S} \rightarrow \pi^{0} \ell^{+} \ell^{-}$}

The measurements of $\operatorname{BR}\left(K_{L} \rightarrow \pi^{0} l^{+} l^{-}\right)$can clarify the mechanism of quark-flavor mixing and $C P$-violation, both within and beyond the SM [206, 211]. In order to extract with high precision the direct $C P$-violating component of the $K_{L}$ amplitude, it is necessary to determine from data the branching fraction of the $C P$-conserving transition $K_{S} \rightarrow \pi^{0} l^{+} l^{-}$.

Any improvement on the present results on $K_{S, \pm} \rightarrow$ $\pi^{0, \pm} \ell^{+} \ell^{-}$is desirable to assess the $C P$-violating term in $K_{L} \rightarrow \pi^{0} e^{+} e^{-}$thus obtaining the SD contribution with the accuracy needed for a significant test of the SM. The $C P$-conserving decays $K^{ \pm}\left(K_{S}\right) \rightarrow \pi^{ \pm}\left(\pi^{0}\right) \ell^{+} \ell^{-}$are dominated by the LD process $K \rightarrow \pi \gamma^{*} \rightarrow \pi \ell^{+} \ell^{-}$[212,213]. The decay amplitudes can be expressed in terms of one form factor $W_{i}(z)\left(i= \pm, S, z=q^{2} / M_{K}^{2}\right)$ which can be decomposed as the sum of a polynomial plus a non-analytic term, $W_{i}^{\pi \pi}(z)$, generated by a $\pi \pi$ loop. The non-analytic term is determined by the $K \rightarrow 3 \pi$ amplitude [213. At $\mathcal{O}\left(p^{6}\right)$ it reads:

$$
W_{i}(z)=G_{F} M_{K}^{2}\left(a_{i}+b_{i} z\right)+W_{i}^{\pi \pi}(z),
$$

where the constants $a_{i}$ and $b_{i}$ parametrize local contributions starting at $\mathcal{O}\left(p^{4}\right)$ and $\mathcal{O}\left(p^{6}\right)$, respectively. The most 
accurate measurements have been obtained with charged kaon beams by BNL-E865 [214] and NA48/2 [215]:

$$
\begin{array}{ll}
a_{+}=-0.587 \pm 0.010 & b_{+}=-0.655 \pm 0.044[214 \\
a_{+}=-0.578 \pm 0.016 & b_{+}=-0.779 \pm 0.066[215
\end{array}
$$

The experimental value of the ratio $b_{+} / a_{+}$is bigger than the estimate from a naive dimensional analysis, $b_{+} / a_{+} \sim$ $\mathcal{O}\left[M_{k}^{2} /\left(4 \pi F_{\pi}\right)^{2}\right] \sim 0.2$, thus pointing to large VMD contributions to the $K \rightarrow \pi l l$ decay .

Chiral symmetry only cannot provide the $K_{S}$ couplings $a_{S}$ and $b_{S}$ in terms of $a_{+}$and $b_{+}$[212,213] and definite predictions on the $K_{S} \rightarrow \pi l l$ mode have to rely on model-dependent assumptions. Vector Dominance hypothesis leads to:

$$
\begin{aligned}
& B R\left(K_{S} \rightarrow \pi^{0} e^{+} e^{-}\right) \approx 5.2 \times 10^{-9} \cdot a_{S}^{2} \\
& B R\left(K_{S} \rightarrow \pi^{0} \mu^{+} \mu^{-}\right) \approx 1.2 \times 10^{-9} \cdot a_{S}^{2}
\end{aligned}
$$

NA48 has obtained first evidence of the $K_{S} \rightarrow \pi l l$ decay for both leptonic channels on the basis of 7 and 6 candidates 216 217 in the $e e$ and $\mu \mu$ mode, respectively. The experimental result on the ratio of the branching fractions is in agreement with Eq. (52) from which they found:

$$
\left|a_{S}\right|_{e e}=1.06_{-0.21}^{+0.26} \pm 0.07 \quad\left|a_{S}\right|_{\mu \mu}=1.54_{-0.32}^{+0.40} \pm 0.06 .
$$

A preliminary KLOE analysis of the $K_{S} \rightarrow \pi^{0} e^{+} e^{-}$based on $480 \mathrm{pb}^{-1}$, an equivalent amount of MC-simulated background and $2 \times 10^{5} \mathrm{MC}$-signal events, has proven that the background sources, mostly from $K_{S} \rightarrow \pi^{0} \pi^{0} \rightarrow \gamma \gamma \gamma e^{+} e^{-}$, can be rejected keeping the overall efficiency to the $5 \%$ level. With the analysis of the entire KLOE-2 data sample we can thus expect a measurement comparable with the NA48 result.

\subsection{4 $K \rightarrow \pi \pi \gamma / K \rightarrow \pi \pi e e$}

Although radiative non-leptonic decays $K_{L}, K_{S} \rightarrow \pi^{+} \pi^{-} \gamma$ and $K^{ \pm} \rightarrow \pi^{ \pm} \pi^{0} \gamma$ are dominated by LD contributions, subleading SD terms in this case can be disentangled studying $C P$-violating observables, useful to test SM predictions 218 .

The $K \rightarrow \pi \pi \gamma$ amplitudes contain two terms, from inner bremsstrahlung, IB, and direct emission, DE. Due to the large contribution from low-energy photons collinear with pions, the IB amplitude is in general much larger than DE. The IB component is suppressed by $C P$-invariance for $K_{L}$ and by $\Delta I=1 / 2$ rule for $K^{ \pm}$, thus allowing the measurement of the DE term in these decays. Electric transitions only are allowed for the IB part, $E_{I B}$, while the DE component can be separated into electric $(E 1)$ and magnetic (M1) dipole terms 219,220. Summing over photon helicities cancels out the interference between electric and magnetic terms. At lowest order, $\mathcal{O}\left(p^{2}\right)$, there is only the bremsstrahlung contribution. At $\mathcal{O}\left(p^{4}\right)$ both $E 1$ and $M 1$ transitions contribute. $E 1$ and $M 1$ are $C P$-allowed for charged kaons while $C P$-simmetry suppresses $E 1$ transitions in the $K_{L}$ decays.
Table 9: Experimental results on $\mathrm{BR}(k \rightarrow \pi \pi \gamma)$ and contributing transitions.

$$
\begin{array}{lcc}
K_{S} \rightarrow \pi^{+} \pi^{-} \gamma & <9 \cdot 10^{-5} & E 1 \\
K^{+} \rightarrow \pi^{+} \pi^{0} \gamma & (0.44 \pm 0.07) 10^{-5} & M 1, E 1 \\
K_{L} \rightarrow \pi^{+} \pi^{-} \gamma & (2.92 \pm 0.07) 10^{-5} & M 1, \mathrm{VMD}
\end{array}
$$

Table 10: Contributions from direct emission and interference $(I N T)$ between IB and DE to radiative $K^{+} \rightarrow \pi^{+} \pi^{0} \gamma$ decays observed by NA48/2 221].

$$
\begin{aligned}
& \begin{array}{cl} 
& T_{\pi}^{*} \in[0,80] \mathrm{MeV} \\
{\frac{\Gamma\left(K^{+} \rightarrow \pi^{+} \pi^{0} \gamma\right)}{\Gamma(T o t)}}_{D E}=\quad(3.35 \pm 0.35 \pm 0.25) \times 10^{-2}
\end{array} \\
& {\frac{\Gamma\left(K^{+} \rightarrow \pi^{+} \pi^{0} \gamma\right)}{\Gamma(T o t)}}_{I N T}=(-2.67 \pm 0.81 \pm 0.73) \times 10^{-2}
\end{aligned}
$$

Present experimental status is summarized in Tab. 9 . NA48/2 has recently obtained first evidence of the $K^{+} \rightarrow$ $\pi^{+} \pi^{0} \gamma$ dipole electric transition, E1, as shown in Tab. 10. The decay rate depends only on $T_{\pi}^{*}$, the kinetic pion energy in the kaon rest frame. Integrating over $T_{\pi}^{*}$, the rate reads:

$$
\frac{d \Gamma}{d W}=\frac{d \Gamma(I B)}{d W} \times\left(1+k_{I N T} \cdot W^{2}+k_{D E} \cdot W^{4}\right),
$$

where $W^{2}=\frac{\left(P_{k}^{*} P_{\gamma}^{*}\right)\left(P_{\pi}^{*} P_{\gamma}^{*}\right)}{\left(m_{K} m_{\pi}\right)^{2}}, P_{i}^{*}$ is the particle 4-momentum and INT denotes the interference between IB- and DEphoton emission. Both $E 1$ from the interference and $M 1$ from the DE term, are extracted from a fit to the W spectrum. The weak counterterms contributing to E1 [198, 199. can be evaluated from the experimental results 221. under the assumption of $E 1$ and $M 1$ amplitudes independent from $W$. The presence of a form factor could affect the distribution and therefore simulate an interference term similar to what observed by NA48/2 [218. The interference term can be further investigated by KLOE2 both with the analysis of $K^{ \pm}$decays and looking at the $K_{S} \rightarrow \pi^{+} \pi^{-} \gamma$ channel which has the same counterterm combination of the weak chiral lagrangian contributing to the DE component. Experimentally, $B R\left(K_{S} \rightarrow\right.$ $\left.\pi^{+} \pi^{-} \gamma\right)(I B) / B R\left(K_{S} \rightarrow \pi^{+} \pi^{-}\right)=6.36 \times 10^{-3}$ with $\mathrm{E}_{\gamma}^{*}>$ $20 \mathrm{MeV}$ while only bounds from E731 exist on the DE component 18:

$$
\begin{aligned}
& B R\left(K_{S} \rightarrow \pi^{+} \pi^{-} \gamma\right)\left(\mathrm{DE}, E_{\gamma}^{*}>50 \mathrm{MeV}\right)<6 \times 10^{-5} \\
& B R\left(K_{S} \rightarrow \pi^{+} \pi^{-} \gamma\right)\left(\mathrm{INT}, E_{\gamma}^{*}>50 \mathrm{MeV}\right)<9 \times 10^{-5} .
\end{aligned}
$$
From theory, $B R\left(K_{S} \rightarrow \pi^{+} \pi^{-} \gamma\right)\left(\right.$ INT, $\left.E_{\gamma}^{*}>20 \mathrm{MeV}\right)=$ $10^{-6} \div 10^{-5}$, reduced by a factor of 2.5 with a $50 \mathrm{MeV}$ cutoff.

The background at KLOE mainly consists of accidental and spurious clusters from machine background and photon splitting, respectively. Both background sources can be safely removed by energy cuts and kinematic contraints coming from completely reconstructed final states. KLOE-2 can select a $K_{S}$ tagged sample of $\sim 3 \times 10^{8}$ events $/ \mathrm{fb}^{-1}$ which corresponds to a total counting of radiative decays of $\sim 8 \times 10^{5} / \mathrm{fb}^{-1}$ with at least $120 / \mathrm{fb}^{-1}$ 
events contributing to the interference. Since the dominant IB term, the interference and the contribution from counterterm combinations have a different dependence on $\mathrm{E}_{\gamma}^{*}$ so that a precision measurement of the photon energy distribution can disentangle the different components. Among other kaon radiative decays, accessible channels at KLOE-2 are the $K \rightarrow \pi \pi e^{+} e^{-}$, particularly the $K_{S} \rightarrow$ $\pi^{+} \pi^{-} e^{+} e^{-}$mode.

NA48 has obtained 222$] \mathrm{BR}\left(K_{s} \rightarrow \pi^{+} \pi^{-} e^{+} e^{-}\right)=(4.69 \pm$ $0.30) \times 10^{-5}$ on the basis of 676 events normalized to the $K_{S} \rightarrow \pi^{0} \pi^{0} \pi_{D}^{0}$, i.e., the kaon decay into three neutral pions followed by the Dalitz decay of one of the pions. With a preliminary analysis based on $\sim 1 \mathrm{fb}^{-1}$, KLOE has obtained 974 cleanly identified events providing the BR measurement with a statistical error of $5 \%$. Differently from NA48, negligible errors are related to the normalization which is done by $K_{S}$ tagging and $K_{S} \rightarrow \pi^{+} \pi^{-}$counting. The evaluation of systematics indicates they could be brought to the per cent level, so that KLOE-2 can reduce the error to $1 \%$. The $K_{S}$ amplitude is dominated by the $C P$-even IB component needed to estimate the $C P$ violating term in the $K_{L}$ decay to the same final state. A test of the $C P$ symmetry in this channel can be performed through the measurement of the angular asymmetry between $\pi \pi$ and $e^{+} e^{-}$planes.

\subsection{Physics of $\eta$ and $\eta^{\prime}$ mesons}

ChPT provides accurate description of the strong and electroweak interactions of the pseudoscalar mesons at low energies 196, 195, 223, 224.

Same final states as in the case of $K_{L}$ can be investigated with the decay of the isospin-singlet $\eta$ meson. Due to weak $K_{L}-\eta$ mixing, understanding $\eta$ decays is a prerequisite for the calculation of SM contributions to rare kaon decays such as $K_{L} \rightarrow \pi^{0} e^{+} e^{-}[212,225]$. CP violation in flavor-conserving processes can be tested in the $\eta$ decays to final states that are, as in the $K_{L}$ case: $\eta \rightarrow \pi \pi$, $\eta \rightarrow \pi^{0} e^{+} e^{-}$and $\eta \rightarrow \pi^{+} \pi^{-} e^{+} e^{-}$decay. In the latter, $C P$ violation could manifest in the angular asymmetry between the $\pi^{+} \pi^{-}$and $e^{+} e^{-}$decay planes.

Conversion decays provide information about transition form-factors needed, e.g., for the light-by-light (LbL) contribution to the anomalous magnetic moment of the muon. The $\eta\left(\eta^{\prime}\right) \rightarrow 3 \pi$ decay is a valuable source of information on light quark masses.

Most complications in treating the $\eta$ in ChPT are due to the mixing of the $\eta$ and $\eta^{\prime}$ fields [226]. The $\eta^{\prime}$ is the most exotic meson of the pseudoscalar nonet, being identified with the $U(1)_{A}$ anomaly singlet $\left(\eta_{0}\right)$ which is massive even in the chiral limit. It was observed by Witten [227] that in QCD with infinite number of colors $\left(\mathrm{N}_{c}\right)$ [228 the $\eta_{0}$ state is massless and the global $S U(3)_{L} \times S U(3)_{R}$ symmetry is replaced by $U(3)_{L} \times U(3)_{R}$. Systematical treatment of the $1 / N_{c}$ expansion, providing foundation for the $\eta-\eta^{\prime}$ mixing, was introduced in ChPT by Kaiser and Leutwyler 229.

The $\eta^{\prime}$ decays to both vector and scalar mesons. The dominance of the vector-meson coupling is demonstrated by radiative decays, $\eta^{\prime} \rightarrow \rho \gamma$ and $\eta^{\prime} \rightarrow \omega \gamma$. Light scalar mesons $\sigma, f_{0}, a_{0}$ should play a significant role in the $\eta \pi \pi$ and $\pi \pi \pi$ decays 230,231,232 although the experimental evidence of the off-shell contributions from large-width scalars is, per se, more elusive. The treatment of the resonances is beyond the scope of standard ChPT, nevertheless continous progress in this field has been achieved over the years. The VMD model, for example, has been incorporated into the theory [233,234 and unitarity constraints included by dispersion relations [235, 236].

In summary, the extension of ChPT stimulated by precision calculations of kaon and $\eta$ decays has also provided elegant and consistent methods for the treatment of $\eta^{\prime}$ physics. The dominant $\eta$ and $\eta^{\prime}$ decays were recently analyzed in the framework of the ChPT extension as in Ref. 237. The amplitudes of hadronic and anomalous decays have been calculated in Refs. 232,238] and Refs. 239,240], respectively.

\subsubsection{Hadronic decays into three pions}

The decay widths of isospin-violating decays $\eta\left(\eta^{\prime}\right) \rightarrow 3 \pi$ are comparable to those of the second-order electromagnetic decays $\eta\left(\eta^{\prime}\right) \rightarrow \gamma \gamma$. The three pion decays are essentially due to the QCD Lagrangian term proportional to $d$ and $u$ quark mass difference, the electromagnetic contribution being suppressed [241,242. The decay width is thus sensitive to the mass difference of the quarks: $\Gamma_{\eta\left(\eta^{\prime}\right) \rightarrow 3 \pi} \propto$ $\Gamma_{0} \times\left(m_{d}-m_{u}\right)^{2}$, and the decay is suitable for precise determination of the ratios of the light quark masses [243]. The calculations of the decay width in ChPT are based on the expansion in the quark masses and the calculations of $\Gamma_{0}$ in the isospin limit. The measurement of both, the value of the decay width, and the Dalitz-plot distribution are needed in order to test ChPT predictions and extract the fractional difference between light quark masses. The decay width is normalized using the two-photon width which can be measured at KLOE-2 in $\gamma \gamma$ interactions, $e^{+} e^{-} \rightarrow e^{+} e^{-} \eta$. The theoretical predictions for $\Gamma_{0}$ are tested comparing the results from the Dalitz plot distributions to the ratio of the two partial widths:

$$
r_{\eta, \eta^{\prime}} \equiv \frac{\Gamma\left(\eta, \eta^{\prime} \rightarrow \pi^{0} \pi^{0} \pi^{0}\right)}{\Gamma\left(\eta, \eta^{\prime} \rightarrow \pi^{+} \pi^{-} \pi^{0}\right)} .
$$

The decay amplitudes for $\eta, \eta^{\prime} \rightarrow \pi^{+} \pi^{-} \pi^{0}$ and $\eta, \eta^{\prime} \rightarrow$ $\pi^{0} \pi^{0} \pi^{0}$ are related by Bose symmetry, assuming $\Delta I=1$ transitions. This implies that, e.g., the relation $r_{\eta, \eta^{\prime}} \leq 3 / 2$ holds in the limit $m_{\pi^{+}}=m_{\pi^{0}}$.

For a three body decay one can use symmetrized, dimensionless variables to parameterize the phase space:

$$
x \equiv \frac{1}{\sqrt{3}} \frac{T_{1}-T_{2}}{\langle T\rangle} ; \quad y \equiv \frac{T_{3}}{\langle T\rangle}-1,
$$

where $T_{1}, T_{2}, T_{3}$ are the pion kinetic energies in the $\eta$ meson rest frame and $\langle T\rangle$ is the average energy: $\langle T\rangle=$ $\left(T_{1}+T_{2}+T_{3}\right) / 3=\left(m_{\eta}-m_{1}-m_{2}-m_{3}\right) / 3$. Decays into three $\pi^{0}$ are conveniently described using polar coordinates $(\sqrt{z}, \phi)$ in the $(x, y)$ plane:

$$
x=\sqrt{z} \sin \phi ; \quad y=\sqrt{z} \cos \phi .
$$


The Dalitz plot has a sextant symmetry so that the range $0^{\circ} \leq \phi<60^{\circ}$ contains all information on the decay. The variable $z$ is given by:

$$
z=x^{2}+y^{2}=\frac{2}{3} \sum_{i=1}^{3}\left(\frac{T_{i}-\langle T\rangle}{\langle T\rangle}\right)^{2}, \quad 0 \leq z \leq 1 .
$$

$\eta \rightarrow 3 \pi$

The neutral decay $\eta \rightarrow 3 \pi^{0}$ has been recently measured by Crystal Ball at BNL 244, KLOE 245, CELSIUS/WASA 246, WASA-at-COSY 247, Crystal Ball at MAMI-B 248 and Crystal Ball at MAMI-C 249. The density of the Dalitz plot is nearly constant and the results of the experiments are expressed by the lowest order expansion at the center:

$$
|\overline{\mathcal{A}}(z, \phi)|^{2}=c_{0}(1+2 \alpha z+\ldots) \text {. }
$$

The experimental values for the $\alpha$ parameter are consistent and the average value is $\alpha=-0.0312 \pm 0.0017$, while ChPT calculations at NNLO, although with large uncertainty, predict a positive value for $\alpha, \alpha=0.013 \pm 0.032$ 250. A better agreement with experimental data was found by model-dependent calculations which include unitarity resummations, giving $\alpha=-0.014$ [236], and $\alpha=$ $-0.031 \pm 0.003$ [238].

The data for $\eta \rightarrow \pi^{+} \pi^{-} \pi^{0}$ decay are dominated by the recent KLOE results with $1.3 \times 10^{6}$ events in the Dalitz plot 251. All other experiments, performed more than thirty years ago, had orders of magnitude lower statistics. The mechanism of the $\eta \rightarrow \pi^{+} \pi^{-} \pi^{0}$ decays is described by an expansion of the amplitude $A(x, y)$ around the center, $x=y=0$, and the density of the Dalitz plot is expressed as:

$$
|\mathcal{A}(x, y)|^{2} \propto 1+a y+b y^{2}+d x^{2}+f y^{3}+\ldots
$$

The LO ChPT amplitude is linear in the $y$ variable with $a=-1.039$ and $b=a^{2} / 4$. From the Dalitz plot density extracted by KLOE, $a=-1.090 \pm 0.005$ (stat) ${ }_{-0.019}^{+0.008}$ (syst) agrees within few per cent with the LO estimate, however higher order ChPT calculations, e.g., $a=-1.371$ at NLO, and $a=-1.271 \pm 0.075$ at NNLO 250, tend to make the agreement worse, for $a$ and for all the parameters describing the $y$-dependence. On the other hand, the $d$ value, $d=0.057 \pm 0.006$ (stat) ${ }_{-0.016}^{+0.007}$ (syst), from KLOE is consistent with $d=0.055 \pm 0.057$ from the NNLO calculations. Experimental uncertainties are lower than the errors on theoretical calculations, nevertheless even higher experimental precision is needed to constrain also the imaginary part of the decay amplitude. The KLOE large drift chamber allowing excellent momentum resolution on $\pi^{ \pm}$ tracks has provided the most precise measurement of the Dalitz-plot distribution of the $\eta \rightarrow \pi^{+} \pi^{-} \pi^{0}$. The analysis of KLOE data is based on $450 \mathrm{pb}^{-1}$ and the sample will be increased by an order of magnitude with the first year of KLOE-2 data-taking. The systematical uncertainty of the
KLOE results is dominated by the event selection procedure. To overcome this limit, new kinds of data-selection criteria will be devised for the larger data samples available.

For the ratio $r_{\eta}$ as in Eq. (155), the ChPT calculation at NNLO 250]) $r_{\eta}=1.47$ is in good agreement with the measurements, $r_{\eta}=1.48 \pm 0.05$ (world average) and $r_{\eta}=1.432 \pm 0.026$ (from the PDG fit) [18. However, the experimental values of the Dalitz-plot parameters, assuming $I=1$ for the final state, lead to $r_{\eta}=1.54$, close to the $\mathrm{LO}$ result in $\mathrm{ChPT}, r_{\eta}=1.52$. Therefore a new precision measurement of the $r_{\eta}$ ratio is needed to get a consistent overall picture of the $\eta \rightarrow 3 \pi$ decays. This requires simultaneous analysis of both $\eta \rightarrow \pi^{+} \pi^{-} \pi^{0}$ and $\eta \rightarrow \pi^{0} \pi^{0} \pi^{0}$ decays leading to cancellation of some of the systematical effects in the ratio.

$\eta^{\prime} \rightarrow 3 \pi$

In the case of $\eta^{\prime}$, it was argued that the decay $\eta^{\prime} \rightarrow \pi \pi \pi$ can be related via $\pi-\eta$ mixing to the isospin-conserving $\eta^{\prime} \rightarrow \eta \pi \pi$ decay and the ratio $B R\left(\eta^{\prime} \rightarrow \pi \pi \pi\right) / B R\left(\eta^{\prime} \rightarrow\right.$ $\eta \pi \pi)$ gives the $\eta-\pi^{0}$ mixing angle or the quark-mass difference 252. This scenario has been recently criticized 253 and it was shown that the relation between the two hadronic decays is more complicated 238] and cannot be expressed by one mixing parameter only. The same work 238 emphasizes that a significant contribution to the $\eta^{\prime} \rightarrow \pi^{+} \pi^{-} \pi^{0}$ from the $\rho^{ \pm} \pi^{\mp}$ intermediate state and thus lower values of the $r_{\eta^{\prime}}$ ratio are expected. A fit to all data (until 2006) on rates and Dalitz plot parameters of $\eta$ and $\eta^{\prime}$ hadronic decays leads to $B R\left(\eta^{\prime} \rightarrow \pi^{+} \pi^{-} \pi^{0}\right) \sim 0.01$ 253. Large contributions from $\rho^{ \pm} \pi^{\mp}$ should also manifest in the Dalitz plot distribution. The $\eta^{\prime} \rightarrow \pi^{0} \pi^{0} \pi^{0}$ decay has been observed by GAMS-2000 254 on the basis of 62 candidates and an expected background of 34 events. The measured partial width of about $300 \mathrm{eV}$ is very close to $\Gamma\left(\eta \rightarrow \pi^{0} \pi^{0} \pi^{0}\right)$ but due to the different total widths for the contributions of isospin-conserving and radiative decays to the $\eta^{\prime}$, it leads to $B R\left(\eta^{\prime} \rightarrow \pi^{0} \pi^{0} \pi^{0}\right)$ $=0.15 \%$. The Dalitz plot slope is consistent with zero within large errors: $\alpha=-0.1 \pm 0.3255$. GAMS-4 $\pi$ has recently collected $235 \pm 45 \eta^{\prime} \rightarrow \pi^{0} \pi^{0} \pi^{0}$ events, obtaining the $B R\left(\eta^{\prime} \rightarrow \pi^{0} \pi^{0} \pi^{0}\right)=(1.8 \pm 0.4) \times 10^{-3}$ and $\alpha=$ $-0.59 \pm 0.18$ 256]. In 2008, CLEO 257 has identified $20.2_{-4.8}^{+6.1} \eta^{\prime} \rightarrow \pi^{+} \pi^{-} \pi^{0}$ decays corresponding to $B R\left(\eta^{\prime} \rightarrow\right.$ $\left.\pi^{+} \pi^{-} \pi^{0}\right)=\left(37_{-9}^{+11} \pm 4\right) \times 10^{-4}$. This result is much lower than predictions from Ref. [253], pointing to a smaller contribution than expected from the $r_{\eta^{\prime}}$ measurement. This is also confirmed by the relatively uniform Dalitz plot distribution found by the same experiment 257. To solve this open question new data on $\eta^{\prime} \rightarrow \pi^{+} \pi^{-} \pi^{0}$ providing stringent constraints on all hadronic $\eta$ and $\eta^{\prime}$ decays [238. 231 are needed. In the first KLOE- 2 run we expect $\approx 8,000$ events produced. The experimental challenge in this case is the background suppression, mostly from $\phi \rightarrow \pi^{+} \pi^{-} \pi^{0}$. 

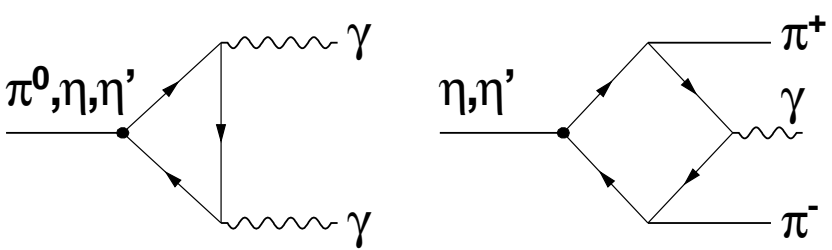

Fig. 10: Diagrams for the triangle and box anomalies.

\subsection{2 $\eta / \eta^{\prime} \rightarrow \pi^{+} \pi^{-} \gamma$}

The decays $\eta / \eta^{\prime} \rightarrow \gamma \gamma$ proceed through the triangle anomaly, and they are accounted for by the Wess-ZuminoWitten (WZW) term into the ChPT Lagrangian. The box anomaly is a higher term of WZW describing the direct coupling of three pseudoscalar mesons with the photon (Fig. 10). The $\eta, \eta^{\prime} \rightarrow \pi^{+} \pi^{-} \gamma$ decays provide a good tool to investigate the box anomaly, which describes the notresonant part of the coupling. The invariant mass of the pions is a good observable to disentangle this contribution from other possible resonant ones, e.g. from the $\rho$-meson. The kinematic range of the $\eta$ and $\eta^{\prime}$ decays is above the chiral limit where the WZW coupling holds. Many efforts have been done to correctly describe contributions of the box anomaly to the two decays. Some examples, which have implemented unitary effects via final-state (FS) interactions, are: HLS, where the hidden local symmetries have been used to evaluate the WZW term 258; the chiral unitary approach using Bethe-Salpeter equation 239; the N/D structure matching the one loop corrections obtained with an Omnes function [259].

The $\eta \rightarrow \pi^{+} \pi^{-} \gamma$ decay has been measured in 1970s 260,261. The analysis of the two data sets, 7,250 and 18,150 events respectively, shows some contradiction. Theoretical papers trying to combine the two measurements have found discrepancies in data treatment and problems with obtaining consistent results 258, 262. Therefore new measurements are needed to clarify the scenario.

KLOE has collected $5 \times 10^{6} \eta \rightarrow \pi^{+} \pi^{-} \gamma$ decays. This sample allows a detailed investigation of the di-pion invariant mass distribution. The main background is due to the $\phi \rightarrow \pi^{+} \pi^{-} \pi^{0}$, with a natural signal to background ratio (S/B) of $1 / 200$. The energy resolution for photons is improved exploiting the kinematic contraints to the $\phi \rightarrow \eta \gamma$ and $\eta \rightarrow \pi^{+} \pi^{-} \gamma$ decays, leading to a final $\mathrm{S} / \mathrm{B}$ of $\sim 20 / 1$ with a selection efficiency on the signal of about $60 \%$. The preliminary analysis of $130 \mathrm{pb}^{-1}$ provides $10^{5}$ signal events. Studies are under way to finalize the selection procedure in order to obtain an efficiency as independent as possible from the $\pi^{+} \pi^{-}$invariant mass and thus an accurate investigation of the box anomaly effect.

Same considerations can be applied to the $\eta^{\prime}$ meson, taking into account that in this case also the $\rho$-resonance has to be considered. Data related to the $\eta^{\prime} \rightarrow \pi^{+} \pi^{-} \gamma$ decays are much more recent: in 1997 the analysis of 7,392 events provided by Crystal Barrel gave evidence for the box anomaly in the invariant mass of pions [263, while in 1998 the L3 collaboration found that their data $(2,123$

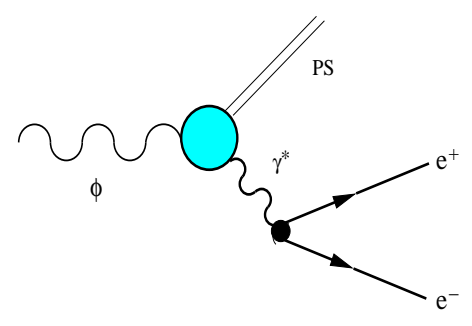

Fig. 11: Diagram of the pseudoscalar production at the $\phi$ factory.

events) were well described by the resonant contribution 264. The relevance of the box anomaly asks for more data, in particular from the next scheduled run of KLOE2 when simultaneous analyses of the $\eta$ and $\eta^{\prime}$ radiative decays can be performed. The data already collected at KLOE contain $1.5 \times 10^{5} \eta^{\prime} \rightarrow \pi^{+} \pi^{-} \gamma$ decays. The feasibility of this measurement has been evaluated on the KLOE sample. With an estimated analysis efficiency of about $20 \%$, it is possible to obtain with KLOE-2/step-0 a result on the basis of $\sim 10^{5}$ selected decays.

\subsubsection{Pseudoscalar form factors}

Pseudoscalar production at the $\phi$ factory (Fig. 11) associated to internal conversion of the photon into a lepton pair allows the measurement of the form factor $\mathcal{F}_{P}\left(q_{1}^{2}=\right.$ $\left.M_{\phi}^{2}, q_{2}^{2}>0\right)$ of the pseudoscalar $P$ in the kinematical region of interest for the VMD model. The vector meson dominance assumption generally provides a good description of the photon coupling to hadrons but in the case of the transition form factor of the $\omega$ meson [265, 266] where standard VMD fails in predicting the strong rise of the coupling in the $\left(0.4<M_{l^{+} l^{-}}<0.6\right) \mathrm{GeV}$ region. Recently, a model for implementing systematic corrections to the standard VMD calculations has been proposed 267 . which correctly describes the $\omega \rightarrow \pi^{0} \mu^{+} \mu^{-}$experimental results, and predicts deviation from standard VMD for the $\phi \rightarrow \eta e^{+} e^{-}$decay spectrum. The only existing data on the latter process come from the SND experiment at Novosibirsk which has measured the $\mathrm{M}_{e e}$ invariant mass distribution on the basis of 74 events [268. The accuracy on the shape does not allow any conclusion on the models. The predictions can be tested with the analysis of KLOE and KLOE-2/step0 data, e.g., selecting the $\eta$ sample by means of the reconstruction of $\eta \rightarrow \pi^{+} \pi^{-} \pi^{0}$ decays. This kind of procedure has been tested on a $90 \mathrm{pb}^{-1}$ sample obtaining, as a figure of merit for the KLOE-2 reach, a clean sample with a selection efficiency of $15 \%$ which translates to 12,000 events $/ \mathrm{fb}^{-1}$.

Pseudoscalar form factors in the time-like region, where $q^{2}>0$, can also be accessed through Dalitz or double Dalitz decays (Fig. 12), which allows the study of $\mathcal{F}_{P}\left(q_{1}^{2}, 0\right)$ and $\mathcal{F}_{P}\left(q_{1}^{2}, q_{2}^{2}\right)$ respectively. Several parametrisations have been proposed, guided by theoretical constraints and phenomenological models. The form factors at $q^{2}>0$ are not well measured and the analysis of both $\pi^{0}$ and $\eta$ meson 


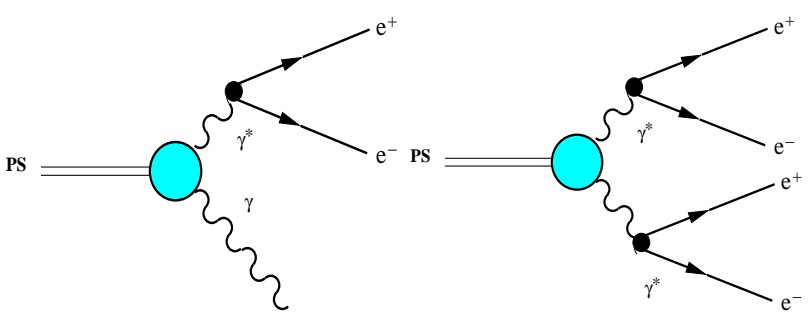

Fig. 12: Diagram for pseudoscalar Dalitz (left) and double Dalitz (right) decays.

Table 11: Measured and expected BRs for $\eta$ into four charged particles.

\begin{tabular}{lcc}
\hline Decay & $\mathrm{BR}_{\exp }$ & $\mathrm{BR}_{\text {theo }}$ \\
\hline$\eta \rightarrow \pi^{+} \pi^{-} e^{+} e^{-}$ & $(26.8 \pm 1.1) 10^{-5}[273]$ & $25.7 \div 36 \cdot 10^{-5}$ \\
$\eta \rightarrow e^{+} e^{-} e^{+} e^{-}$ & $<6.9 \cdot 10^{-5}[274]$ & $2.5 \div 2.6 \cdot 10^{-5}$ \\
$\eta \rightarrow \mu^{+} \mu^{-} e^{+} e^{-}$ & - & $1.6 \div 2.2 \cdot 10^{-5}$ \\
$\eta \rightarrow \pi^{+} \pi^{-} \mu^{+} \mu^{-}$ & - & $8 \cdot 10^{-7}$ \\
\hline
\end{tabular}

decays deserves more efforts. For the $\pi^{0}$, both $\mathcal{F}_{\pi^{0}}\left(q^{2}, 0\right)$ and $\mathcal{F}_{\pi^{0}}\left(q_{1}^{2}, q_{2}^{2}\right)$ are extracted with large errors [269, 270], while in the $\eta$ case only the $\eta \rightarrow \mu^{+} \mu^{-} \gamma$ form factor has been measured [271,266].

A copious number of $\pi^{0}$ will be produced at KLOE-2 through the decays $\phi \rightarrow \pi^{+} \pi^{-} \pi^{0}$ and $K_{L} \rightarrow \pi^{+} \pi^{-} \pi^{0}$. For both processes the $\pi^{0}$ momentum can be obtained from precision measurements of the charged-particle tracks. In the first case the event topology is simpler and the $\pi^{0}$ momentum can be determined with $<1 \mathrm{MeV}$ resolution using the measured $\pi^{+}$and $\pi^{-}$tracks and energy-momentum conservation. The $\pi^{0}$ decay vertices at the IP are are precisely and efficiently reconstructed. The sample can be enlarged using the $K_{L} \rightarrow \pi^{+} \pi^{-} \pi^{0}$ decay, even though the resolution on $K_{L}$ momentum and vertex position are worse than in the $\phi \rightarrow \pi^{+} \pi^{-} \pi^{0}$ mode. With $10 \mathrm{fb}^{-1}$ taking into account the analysis efficiency, a sample of $10^{8}$ $\pi^{0} \rightarrow e^{+} e^{-} \gamma$ and $180,000 \pi^{0} \rightarrow e^{+} e^{-} e^{+} e^{-}$events is expected, larger than what has been used in previous experiments.

In the $\eta$ case we expect $3 \times 10^{5} \eta \rightarrow e^{+} e^{-} \gamma$ and $2.8 \times 10^{4} \eta \rightarrow e^{+} e^{-} e^{+} e^{-}$events. As a figure of merit of the sensitivity to the $\eta$ form factors with the first KLOE-2 run, in Fig. 13 the $m_{e^{+} e^{-}}$distribution of the $\eta \rightarrow e^{+} e^{-} \gamma$ events is shown for point-like and pole approximation of the $\mathcal{F}_{\eta}\left(q^{2}, 0\right)$ form factor, assuming an overall analysis efficiency of $10 \%$. KLOE-2 can also provide the first measurement of the $m_{e^{+} e^{-}}$distribution from the analysis of the four-electrons mode which is expected to be almost independent from the form factor parametrisation 272 .

\subsection{4 $\eta$ decays into four charged particles}

With $\mathcal{O}(10) \mathrm{fb}^{-1}$ KLOE-2 can access the first three decays summarized in Tab.11, These decays allow the study of the $\eta$ meson internal structure, measuring the virtual-

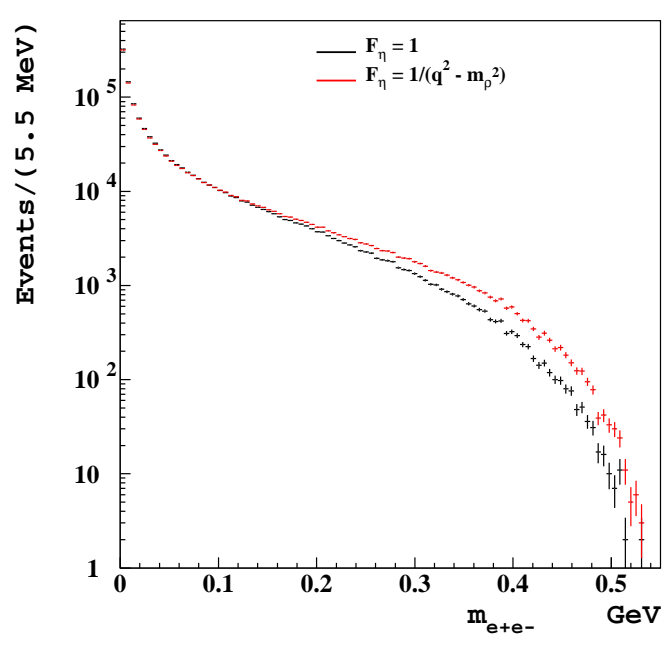

Fig. 13: The $m_{e^{+} e^{-}}$distribution of $\eta \rightarrow e^{+} e^{-} \gamma$ events, for point-like and pole approximation of the $\mathcal{F}_{\eta}\left(q^{2}, 0\right)$ form factor.

photon 4-momentum via the invariant mass of the lepton pair [275]. This is important for the connections with light-by-light physics 272. In particular, the comparison of the kinematical distributions of the $\eta \rightarrow e^{+} e^{-} e^{+} e^{-}$to $\eta \rightarrow \mu^{+} \mu^{-} e^{+} e^{-}$can provide significant information on the underlying processes. These decays can also probe some physics beyond the SM: CP violation in $\eta \rightarrow \pi^{+} \pi^{-} e^{+} e^{-}$ [276] and light CP-odd Higgs of the NMSSM (next-tominimal supersymmetric SM) in $\eta \rightarrow \pi^{+} \pi^{-} \mu^{+} \mu^{-}$[277]. The latter could provide up to 1-2 events per $\mathrm{fb}^{-1}$, not sufficient to be investigated at KLOE-2. The $\eta \rightarrow \pi^{+} \pi^{-} e^{+} e^{-}$ decay has been already studied with KLOE [273, which measured an asymmetry $A_{\phi}$ in the angle between the $\pi \pi$ and ee decay planes consistent with zero to the $3 \%$ precision level, while theoretical predictions allow this quantity to be up to $2 \%$. The largest contribution to the uncertainty of this measurement comes from the statistical error. In KLOE the minimal transverse momentum of reconstructed tracks, $P_{T_{\min }}$, is $23 \mathrm{MeV}$. It limits the selection efficiency to $\sim 8 \%$. The installation of the inner tracker, in the second phase of the KLOE-2 experiment would reduce $P_{T_{\min }}$ to $16 \mathrm{MeV}$ improving at the same time the tracking resolution, beneficial for the rejection of photon-conversion events mimicking the signal [273. With a sample of $20 \mathrm{fb}^{-1}$ and the increase in the efficiency from both acceptance and easier background rejection, KLOE-2 could obtain $A_{\phi}$ with $0.8-1 \%$ precision.

$$
\text { 4.2.5 } \eta \rightarrow \pi^{0} \gamma \gamma
$$

The $\eta \rightarrow \pi^{0} \gamma \gamma$ decay is very interesting both from the experimental and theoretical point of view. Big theoretical efforts to evaluate the $B R\left(\eta \rightarrow \pi^{0} \gamma \gamma\right)$ were originally motivated by the large branching fraction obtained by experiments dating back 40 years. ChPT terms 278 

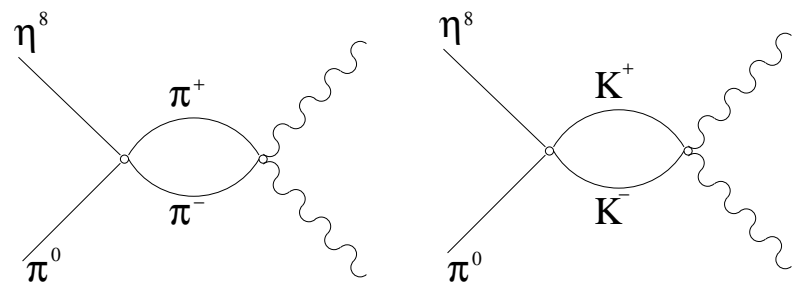

Fig. 14: Meson loop diagrams in the $\eta \rightarrow \pi^{0} \gamma \gamma$ decay.

start at order $\mathcal{O}\left(p^{4}\right)$ through the pion and kaon loop diagrams shown in Fig. 14. The $\eta^{8} \pi^{0} \pi^{+} \pi^{-}$vertex and kaonloop diagrams are suppressed by G-parity conservation and the large kaon mass, respectively. Therefore, relatively sizable contributions at higher order are expected. At $\mathcal{O}\left(p^{6}\right)$ the counterterms of the chiral lagrangian can not be determined by experimental input only so that the prediction of the decay rate goes beyond pure ChPT constrained by experimental data. In Ref. 278 large $N_{C}$ expansion and resonance saturation were used to determine the $\mathcal{O}\left(p^{6}\right)$ LECs in the Lagrangian, obtaining $\Gamma(\eta \rightarrow$ $\left.\pi^{0} \gamma \gamma\right)=0.18 \mathrm{eV}$. The contribution from $a_{0}(980)$ was also estimated, $\Gamma\left(\eta \rightarrow \pi^{0} \gamma \gamma\right)_{a_{0}}=0.02 \mathrm{eV}$ but the relative phase with respect to the VMD term is unknown. The uncertainty from the sign of the interference term is quoted as the dominant contribution to the theoretical error at $\mathcal{O}\left(p^{6}\right), \Gamma\left(\eta \rightarrow \pi^{0} \gamma \gamma\right)=0.18 \pm 0.02 \mathrm{eV}$ or $B R\left(\eta \rightarrow \pi^{0} \gamma \gamma\right)=(1.40 \pm 0.14) \times 10^{-4}$. Higher order terms in ChPT have been also studied, e.g. in Ref. 278 where all-order VMD counterterms have been added, obtaining $\Gamma\left(\eta \rightarrow \pi^{0} \gamma \gamma\right)=(0.42 \pm 0.2) \mathrm{eV}$ with the uncertainty again mostly from the sign of the interference term. On the experimental side, several measurements have been published since 1970s with smaller and smaller values of the branching fraction, decreased by three order of magnitude from 1967 to 1981 when the GAMS-2000 experiment measured $B R\left(\eta \rightarrow \pi^{0} \gamma \gamma\right)=(7.1 \pm 1.4) \times 10^{-4}$. In 2005 the AGS/Crystal Ball collaboration has obtained an even lower value, $B R\left(\eta \rightarrow \pi^{0} \gamma \gamma\right)=(3.5 \pm 0.9) \times 10^{-4}$, updated to $B R\left(\eta \rightarrow \pi^{0} \gamma \gamma\right)=(2.2 \pm 0.5) \times 10^{-4}$ in year 2008 with a revised analysis aiming at the optimization of the selection criteria and fitting procedure of the experimental spectra. The prelimary KLOE result 279 is $B R(\eta \rightarrow$ $\left.\pi^{0} \gamma \gamma\right)=(8 \pm 3) \times 10^{-5}, 1.8-\sigma$ lower than the ChPT prediction at $\mathcal{O}\left(p^{6}\right)$ and 2.2- $\sigma$ lower than the AGS/CB measurement. Using the KLOE preliminary result on the branching fraction and the analysis efficiency obtained of $\sim 5 \%$, $1,300 \eta \rightarrow \pi^{0} \gamma \gamma$ events are expected from the first year of data-taking at KLOE-2, thus allowing an accuracy of $3 \%$ . Moreover, KLOE-2 can provide the the $m_{\gamma \gamma}$ distribution with sufficient precision to solve the ambiguity connected to the sign of the interference between VMD and scalar terms as shown in Fig. 15.

\subsection{6 $\eta-\eta^{\prime}$ mixing and gluonium content}

The $\eta^{\prime}$ meson, being almost a pure $\mathrm{SU}(3)_{\text {Flavor }}$ singlet, is considered a good candidate to host a gluon condensate.

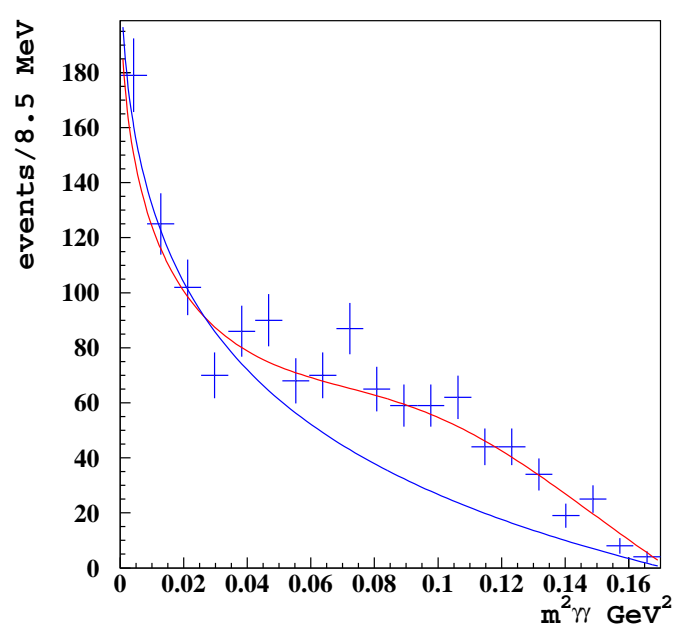

Fig. 15: The $m_{\gamma \gamma}^{2}$ distribution in $\eta \rightarrow \pi^{0} \gamma \gamma$ decays expected at KLOE- 2 for the VMD $+a_{0}(980)$ model, with constructive and destructive interference term. Crosses are the simulated experimental data assuming $5 \%$ constant efficiency as a function of $m_{\gamma \gamma}^{2}$ and constructive interference.

KLOE 280 has extracted the $\eta^{\prime}$ gluonium content and the $\eta-\eta^{\prime}$ mixing angle according to the model of Ref. 281. The $\eta$ and $\eta^{\prime}$ wave functions can be decomposed in three terms: the $u, d$ quark wave function $|q \bar{q}\rangle=\frac{1}{\sqrt{2}}(|u \bar{u}\rangle+|d \bar{d}\rangle)$, the $|s \bar{s}\rangle$ component and the gluonium $|G G\rangle$. The wave functions are written as:

$$
\begin{aligned}
\left|\eta^{\prime}\right\rangle & =\cos \psi_{G} \sin \psi_{P}|q \bar{q}\rangle+\cos \psi_{G} \cos \psi_{P}|s \bar{s}\rangle+\sin \psi_{G}|G G\rangle \\
|\eta\rangle & =\cos \psi_{P}|q \bar{q}\rangle-\sin \psi_{P}|s \bar{s}\rangle
\end{aligned}
$$

where $\psi_{P}$ is the $\eta-\eta^{\prime}$ mixing angle and $Z_{G}^{2}=\sin ^{2} \psi_{G}$ is the gluonium fraction in the $\eta^{\prime}$ meson. The $Z_{G}^{2}$ parameter can be interpreted as the mixing with a pseudoscalar glueball. In Ref. 282 it has been identified with the $\eta(1405)$. The gluonium fraction has been extracted fitting the widths of the magnetic dipole transition $V \rightarrow P \gamma$, where $V$ are the vector mesons $\rho, \omega, \phi$ and $P$ the pseudoscalar mesons $\pi^{0}, \eta, \eta^{\prime}$, together with the $\pi^{0} \rightarrow \gamma \gamma$ and $\eta^{\prime} \rightarrow \gamma \gamma$ partial widths. In particular, the KLOE measurement of $R_{\phi}=$ $B R\left(\phi \rightarrow \eta^{\prime} \gamma\right) / B R(\phi \rightarrow \eta \gamma)$ has been used, related to the gluonium content and mixing angle by [283]:

$$
R_{\phi}=\cot ^{2} \psi_{P} \cos ^{2} \psi_{G}\left(1-\frac{m_{s}}{\bar{m}} \frac{Z_{q}}{Z_{s}} \frac{\tan \psi_{V}}{\sin 2 \psi_{P}}\right)^{2}\left(\frac{p_{\eta^{\prime}}}{p_{\eta}}\right)^{3}
$$

where $p_{\eta^{\prime}}$ and $p_{\eta}$ are the momenta of the $\eta^{\prime}$ and $\eta$ meson respectively, $m_{s} / \bar{m}=2 m_{s} /\left(m_{u}+m_{d}\right)$ is the mass ratio of the constituent quarks and $\psi_{V}$ is the $\phi-\omega$ mixing angle. The $Z_{q}$ and $Z_{s}$ parameters take into account hadronic uncertainties 284. The evidence at $3-\sigma$ level of a gluonium component in the $\eta^{\prime}$ has been obtained, $Z_{G}^{2}=(12 \pm 4) \%$. A detailed study of the fit has shown that the measurement 
Table 12: Fit to the gluonium content in $\eta^{\prime}$ assuming $1 \%$ error on the $\eta^{\prime}$ branching fractions. The $\eta^{\prime} \rightarrow \gamma \gamma / \pi^{0} \rightarrow \gamma \gamma$ constraint is used (not used) in the left (right) column.

\begin{tabular}{c|c|c} 
& with $\eta^{\prime} \rightarrow \gamma \gamma / \pi^{0} \rightarrow \gamma \gamma$ & without $\eta^{\prime} \rightarrow \gamma \gamma / \pi^{0} \rightarrow \gamma \gamma$ \\
\hline$Z_{G}^{2}$ & $0.11 \pm 0.03$ & $0.11 \pm 0.04$ \\
$\psi_{P}$ & $(40.5 \pm 0.6)^{\circ}$ & $40.5 \pm 0.6$ \\
$Z_{N S}$ & $0.93 \pm 0.02$ & $0.93 \pm 0.03$ \\
$Z_{S}$ & $0.83 \pm 0.05$ & $0.83 \pm 0.05$ \\
$\psi_{V}$ & $(3.32 \pm 0.08)^{\circ}$ & $(3.32 \pm 0.09)$ \\
$m_{s} / \bar{m}$ & $1.24 \pm 0.07$ & $1.24 \pm 0.07$ \\
\hline
\end{tabular}

of the ratio $\Gamma\left(\eta^{\prime} \rightarrow \gamma \gamma\right) / \Gamma\left(\pi^{0} \rightarrow \gamma \gamma\right)$ is the most sensitive to the gluonium fraction.

The theoretical frameworks to explain $\eta^{\prime} \rightarrow \gamma \gamma$ and $V \rightarrow P \gamma$ transitions are slightly different, the first being related to quark-antiquark annihilation to two photons, the second to spin-flip transitions between the vector and pseudoscalar meson. Therefore it is important to obtain both, a sensitivity to the gluonium fraction independent from the $\eta^{\prime} \rightarrow \gamma \gamma$ decay, and a new measurement of the $\eta^{\prime} \rightarrow \gamma \gamma$ branching fraction. The $\eta^{\prime} \rightarrow \gamma \gamma$ branching fraction is $\sim 2 \%$. Using $\operatorname{BR}\left(\phi \rightarrow \eta^{\prime} \gamma\right) \sim 6 \times 10^{-5}$ and $\sigma\left(e^{+} e^{-} \rightarrow \phi\right)=3.3 \mu \mathrm{b}$, we expect 4 events $/ \mathrm{pb}^{-1}$. Unluckily this signal is overwhelmed by the $e^{+} e^{-} \rightarrow \gamma \gamma$ background. With the available sample after one year running at KLOE-2, we will able to perform a selection based 40,000 events, sufficient to improve the fractional accuracy on $\operatorname{BR}\left(\eta^{\prime} \rightarrow \gamma \gamma\right)$ to the per cent level.

The $B R\left(\eta^{\prime} \rightarrow \gamma \gamma\right)$ limits the accuracy on the $\eta^{\prime}$ decay width while the $\eta^{\prime} \rightarrow \pi^{+} \pi^{-} \eta$ and the $\eta^{\prime} \rightarrow \pi^{0} \pi^{0} \eta$ branching ratios dominate the systematic error on the $R_{\phi}$ measurement because the $\eta^{\prime}$ is identified through these decays. Table 12 shows how the evidence for a gluonium content in the $\eta^{\prime}$ could be confirmed also independently from the $\Gamma\left(\eta^{\prime} \rightarrow \gamma \gamma\right) / \Gamma\left(\pi^{0} \rightarrow \gamma \gamma\right)$ ratio if the fractional accuracy on the $\eta^{\prime}$ partial widths is brought to $1 \%$ level. We have assumed that the actual branching fractions and the correlation matrix of the measurements are those obtained from the present fit.

With the KLOE-2 data-taking above the $\phi$ peak, e.g., at $\sqrt{s} \sim 1.2 \mathrm{GeV}$, it is possible to measure the $\eta^{\prime}$ decay width $\Gamma\left(\eta^{\prime} \rightarrow \gamma \gamma\right)$ through $\sigma\left(e^{+} e^{-} \rightarrow e^{+} e^{-}\left(\gamma^{*} \gamma^{*}\right) \rightarrow\right.$ $\left.e^{+} e^{-} \eta^{\prime}\right)$, as discussed in Sect. 6. The measurement to $1 \%$ level of both the cross section and the $B R\left(\eta^{\prime} \rightarrow \gamma \gamma\right)$ would bring the fractional error on the $\eta^{\prime}$ total width, $\Gamma_{\eta^{\prime}}=\Gamma\left(\eta^{\prime} \rightarrow \gamma \gamma\right) / B R\left(\eta^{\prime} \rightarrow \gamma \gamma\right)$, to $\sim 1.4 \%$. In Fig. 16 the $68 \%$ C.L. region in the $\psi_{P}, Z_{G}^{2}$ plane obtained with the improvements discussed in this subsection is shown. The comparison of the top to bottom panels makes evident how the fit accuracy increases with the precision measurement of the $\eta^{\prime}$ total width.

\subsection{Low-Mass Scalars}

The radiative processes $\phi \rightarrow \gamma S$, where $S=f_{0}(980)$, $a_{0}(980), \sigma \equiv f_{0}(600)$, are followed by a decay of $S$ into
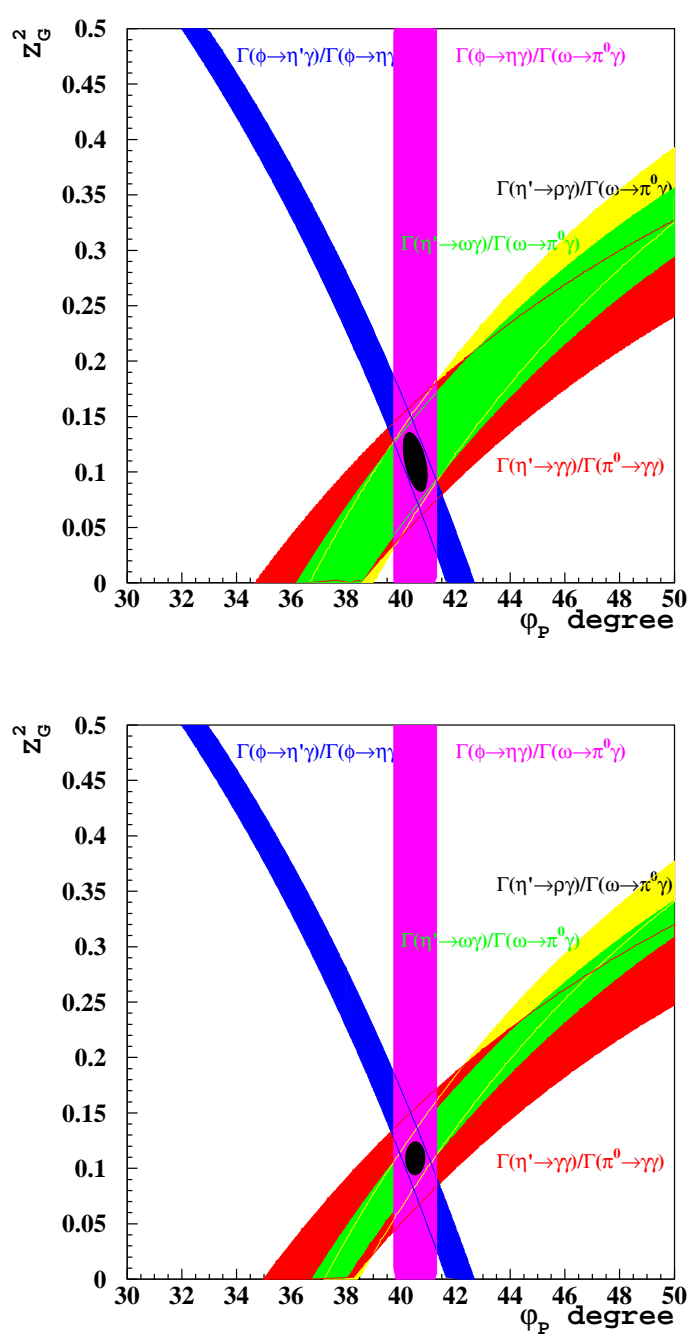

Fig. 16: The $68 \%$ C.L. region in the $\psi_{P}, Z_{G}^{2}$ plane. Top: only the $\eta^{\prime}$ branching ratios are improved to $1 \%$ precision. Bottom: the $\eta^{\prime}$ total width is also lowered to $1.4 \%$.

two pseudoscalar mesons $(P P)$. The analysis of the $M_{P P}$ invariant mass distribution in $\phi \rightarrow \gamma P P$ transitions is sensitive to the nature of light scalar states. If they are tetraquarks, quarkonia or molecules is one of the open questions of low-energy QCD. Interestingly, the tetraquark assignment 285, 286 naturally explains the mass pattern and decay widths, although different instanton-driven [287] or quark-antiquark processes 288] have been considered.

Two kinds of theoretical models for the $\phi \rightarrow \gamma S \rightarrow$ $\gamma P P$ have been analyzed: (i) the 'no structure' approach based on a point-like $\phi \gamma S$ coupling 289,290, 291]; (ii) the kaon-loop coupling of the $\phi$ to $\gamma S$ [292. Future analyses will determine which way is dominant and the decay amplitudes of the scalar states. Moreover, the precision study of the $S \rightarrow \gamma \gamma$ reactions at KLOE-2 as presented in Sect. 6] would add information relevant for the underlying quark dynamics. 
The solution of the scalar puzzle, which has stimulated many other theoretical studies, e.g. [293, 294,295, 296, can also shed light on the mechanism of chiral symmetry restoration at non-zero temperature and density, where scalar mesons, which carry the quantum numbers of the vacuum, play a central role 297.

\subsubsection{Structure of $f_{0}(980)$ and $a_{0}(980)$}

The KLOE experiment has measured the branching ratio and the spectral function of the decays $\phi \rightarrow S \gamma \rightarrow$ $\pi^{0} \pi^{0} \gamma, \quad \pi^{0} \eta \gamma, \quad \pi^{+} \pi^{-} \gamma$ 298, 299, 300 obtaining within the Kaon Loop phenomenological model the coupling of scalar mesons to the $\pi \pi$ and $\eta \pi^{0}$ pairs. While in the latter only the $a_{0}(980)$ can contribute, in the $\pi \pi$ channel both, the $f_{0}(980)$, and the $\sigma$ whose existence has been debated since a long time, are expected. KLOE has fitted the $\pi^{0} \pi^{0}$ invariant mass fixing the parameters of the $\sigma$ meson from other measurements and phenomenological fits. Data from $\pi^{+} \pi^{-}$phase shift were also taken into account. For the charged channel, due to the large background from the $e^{+} e^{-} \rightarrow \pi^{+} \pi^{-} \gamma$ dominated by the $\rho$ pole, no sensitivity to the $\sigma$ meson is shown by the fit. The present analysis of KLOE data is based on $\sim 500 \mathrm{pb}^{-1}$. KLOE- 2 will have a 20 times larger sample, so that the $\sigma$ could also show up in the $\pi^{+} \pi^{-}$channel. Moreover, the $\pi^{+} \pi^{-}$forwardbackward asymmetry $\left(A_{F B}\right)$ is expected to be affected by the presence of the $\sigma$. This asymmetry is generated by the different behaviour under C-conjugation of the $\pi^{+} \pi^{-}$ amplitude for initial state radiation (ISR) with respect to the other contributions (final state radiation (FSR) and $e^{+} e^{-} \rightarrow \phi \rightarrow\left(f_{0}(980)+\sigma\right) \gamma$. In Fig. 17 $A_{F B}$ is shown for two sets of KLOE data, collected at $\sqrt{s}=m_{\phi}$ and $\sqrt{s}=1$ $\mathrm{GeV}$. In the second case no scalar should be present due to the low value of the $e^{+} e^{-} \rightarrow \phi$ cross section. A combined fit to the invariant mass spectrum and asymmetry is of interest at KLOE-2 for the study of the properties of the $f_{0}(980)$ and $\sigma$ mesons.

The set of scalar couplings to the pseudoscalars can be used to determine the structure of the scalar meson in the naive $S U(3)$ hypothesis. In Tab. 13, the $\mathrm{SU}(3)$ predicted couplings with different quark structure of the pseudoscalars is shown compared with the KLOE results. The 4-quark model predicts values larger than observed for $g_{a_{0} K^{+} K^{-}} / g_{a_{0} \eta \pi}$ while the 2 -quark model can be accommodated only if $f_{0}=s \bar{s}$, which is disfavoured by the mass degeneracy of the two mesons. The ratio of $f_{0}$ to $a_{0}$ couplings to $K^{+} K^{-}$is higher with respect to all predictions.

\subsubsection{Structure of the $\sigma$ meson}

In the $\eta^{\prime} \rightarrow \pi^{+} \pi^{-} \eta$ and $\eta^{\prime} \rightarrow \pi^{0} \pi^{0} \eta$, the $\pi \pi$ system is produced mostly with scalar quantum numbers. Indeed, the available kinetic energy of the $\pi^{+} \pi^{-}$pair is $[0,137]$ $\mathrm{MeV}$, suppressing high angular momentum contribution. Furthermore, the exchange of vector mesons is forbidden by G-parity conservation. For these reasons, only scalar mesons can participate to the scattering amplitude. The
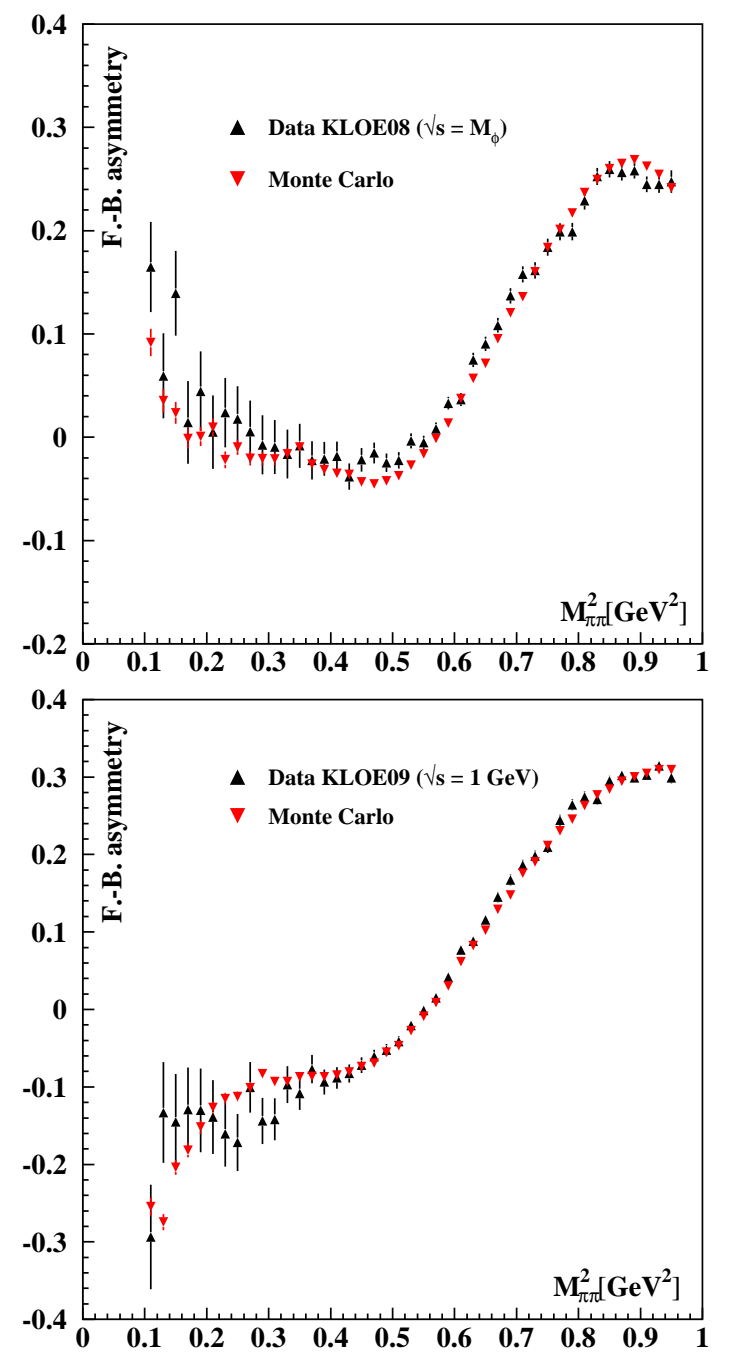

Fig. 17: Forward-backward asymmetry, $A_{F B}$, as a function of $M_{\pi \pi}^{2}$ in $e^{+} e^{-} \rightarrow \pi^{+} \pi^{-} \gamma$ events for data at $\sqrt{s}=m_{\phi}$ (top) and at $\sqrt{s}=1 \mathrm{GeV}$ (bottom).

decay can be mediated by the $\sigma, a_{0}(980)$ and $f_{0}(980)$ exchange and by a direct contact term due to the chiral anomaly 230. The scalar contribution can be determined by fitting the Dalitz plot of the $\eta^{\prime} \rightarrow \pi \pi \eta$ system. The golden channel for KLOE-2 is the decay chain $\eta^{\prime} \rightarrow \pi^{+} \pi^{-} \eta$, with $\eta \rightarrow \gamma \gamma$. The signal can be easily identified requiring the $\eta$ and $\eta^{\prime}$ invariant masses. Such final state was already studied at KLOE to measure the branching fraction of the $\phi \rightarrow \eta^{\prime} \gamma$ decay 301. The analysis efficiency was $22.8 \%$, with $10 \%$ residual background contamination. With $\mathcal{O}(10) \mathrm{fb}^{-1}$, we expect 80,000 fully reconstructed events. Following Ref. 230 we have written the matrix element of the decay and built a MC generator. In Fig. 18 the $m_{\pi^{+} \pi^{-}}$invariant mass distribution is shown with and without the $\sigma$ contribution with the expected KLOE-2 statistics. Good sensitivity to the parameters of the $\sigma$ meson is therefore expected from the study of this channel. 
Table 13: Comparison of the measured couplings to the SU(3) predictions for different quark-structure hypotheses.

\begin{tabular}{|c|c|c|c|c|}
\hline & KLOE & \multicolumn{3}{|c|}{$\mathrm{SU}(3)$} \\
\hline & & $4 \mathrm{q}$ & $\mathrm{q} \bar{q} f_{0}=s \bar{s}$ & $q \bar{q} f_{0}=(u \bar{u}+d \bar{d}) / \sqrt{2}$ \\
\cline { 3 - 5 } & & $1.2-1.7$ & 0.4 & 0.4 \\
$\left(g_{a_{0} K^{+} K^{-}} / g_{a_{0} \eta \pi}\right)^{2}$ & $0.6-0.7$ & $1 / 4$ \\
$\left(g_{f_{0} K^{+} K^{-}} / g_{f_{0} \pi^{+} \pi^{-}}\right)^{2}$ & $4.6-4.8$ & $>>1$ & $>>1$ & 1 \\
$\left(g_{f_{0} K^{+} K^{-}} / g_{a_{0} K^{+} K^{-}}\right)^{2}$ & $4-5$ & 1 & 2 & \\
\hline
\end{tabular}

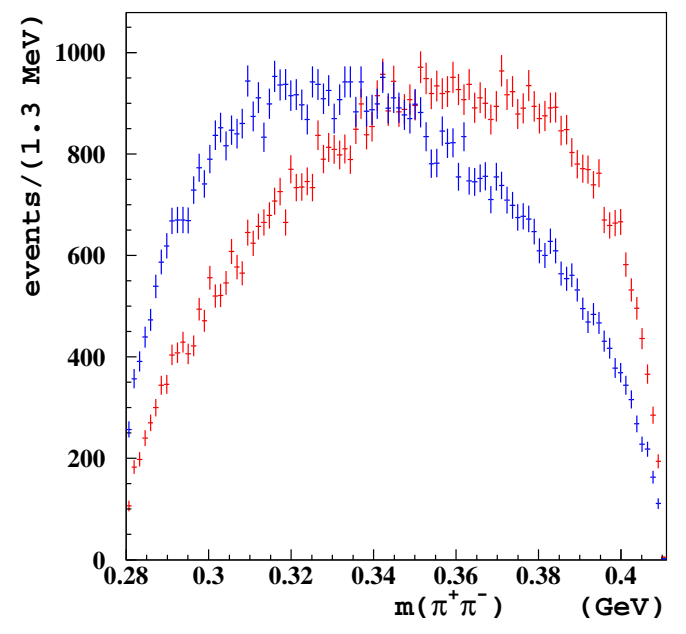

Fig. 18: The $m_{\pi^{+} \pi^{-}}$distribution in the $\eta^{\prime} \rightarrow \eta \pi^{+} \pi^{-}$decay with the $\sigma$ meson (right-centered distribution) and without (left-centered distribution) contribution.

\subsection{3 $\phi \rightarrow K^{0} \bar{K}^{0} \gamma$}

The $\phi$ meson can decay to the $a_{0}(980)$ and $f_{0}(980)$ scalars with the emission of one photon. Both of them couples to $K^{+} K^{-}$and $K^{0} \bar{K}^{0}$, so that the decay $\phi \rightarrow K^{0} \bar{K}^{0} \gamma$ is expected to proceed through an $\left[f_{0}(980)+a_{0}(980)\right] \gamma$ intermediate state. $\mathrm{SU}(2)$ relates the coupling constant to the pseudoscalars in a clean way: $g_{f_{0} \pi^{+} \pi^{-}}=2 g_{f_{0} \pi^{0} \pi^{0}}$, $g_{f_{0} K^{0} \bar{K}^{0}}=g_{f_{0} K^{+} K^{-}}, g_{a_{0} K^{0} \bar{K}^{0}}=-g_{a_{0} K^{+} K^{-}}$. Due to the opposite sign in $g_{f_{0} K^{0} \bar{K}^{0}} \cdot g_{f_{0} K^{+} K^{-}}$and $g_{a_{0} K^{0} \bar{K}^{0}} \cdot g_{a_{0} K^{+} K^{-}}$, destructive interference between $f_{0}(980)$ and $a_{0}(980)$ is expected. The scalars decay in an even combination of $\left|K^{0}\right\rangle\left|\bar{K}^{0}\right\rangle: \frac{\left.\left|K^{0}>\bar{K}^{0}>+\right| \bar{K}^{0}>K^{0}\right\rangle}{\sqrt{2}}=\frac{\left.\left|K_{S}>\right| K_{S}\right\rangle+\left|K_{L}>K_{L}\right\rangle}{\sqrt{2}}$. The channel with two $K_{S}$ in the final state can be easily identified through the $K_{S} \rightarrow \pi^{+} \pi^{-}$decay, looking for 4 tracks pointing to the IP. The main background comes from $\phi \rightarrow$ $K_{S} K_{L}$ events with a $C P$-violating decay $K_{L} \rightarrow \pi^{+} \pi^{-}$. In KLOE, the $K_{S}$ decay path is $6 \mathrm{~mm}$, while for the $K_{L}$ is $\sim 3.4 \mathrm{~m}$. Therefore the $K_{L}$ vertices are uniformly distribuited in a small region around the IP. Cuts on the vertex position are the most effective way to remove $K_{S} K_{L}$ background. KLOE 302 has already analyzed a sample of $2.2 \mathrm{fb}^{-1}$ of data, observing 5 events with $3.2 \pm 0.7$ expected background. The result is $B R\left(\phi \rightarrow K^{0} \bar{K}^{0} \gamma\right)<1.9 \times 10^{-8}$, at $90 \%$ C.L.. Scaling these numbers with the KLOE-2 statistics we expect to reach a sensitivity of $B R(\phi \rightarrow$ $\left.K^{0} \bar{K}^{0} \gamma\right)<1 \times 10^{-8}$. The inner tracker will provide in the second phase of the experiment three times better vertex resolution which is beneficial for the rejection capability and together with $20 \mathrm{fb}^{-1}$ of integrated luminosity could lead to first observation of the decay.

\section{Physics in the Continuum: $\sigma_{\text {had }}$}

In this section we discuss the physics reach of the DA $\Phi$ NE running outside the $\phi$-meson peak. We consider a maximal energy of $\sqrt{s}=2.5 \mathrm{GeV}$ with a luminosity of $\sim 10^{32}$ $\mathrm{cm}^{-2} \mathrm{~s}^{-1}$ already exceeded by DA $\Phi$ NE at the $\phi$ peak. With such a machine one can collect an integrated luminosity of $\mathcal{O}(10) \mathrm{fb}^{-1}$ between 1 and $2.5 \mathrm{GeV}$ in a few years of data taking. This high statistics, much larger than what collected at any collider in this energy range, would allow major improvements in physics, with relevant implications for the precision tests of the SM, such as the $g-2$ of the muon and the effective fine-structure constant at the $M_{Z}$ scale, $\alpha_{e m}\left(M_{Z}^{2}\right)$. The only direct competitor is VEPP-2000 at Novosibirsk, which will cover the center-ofmass energy range between 1 and $2 \mathrm{GeV}$ with two experiments. VEPP-2000 is expected to start by year 2010 with a luminosity from $10^{31} \mathrm{~cm}^{-2} \mathrm{~s}^{-1}$ at $1 \mathrm{GeV}$ to $10^{32} \mathrm{~cm}^{-2} \mathrm{~s}^{-1}$ at $2 \mathrm{GeV}$, as presented in more detail in Sect. 5.6. Other indirect competitors are the higher-energy $e^{+} e^{-}$colliders ( $\tau$-charm and B-factories) which in principle can cover the same energy range by means of radiative return. However, due to the photon emission, the "equivalent" luminosity produced by these machines in the region between 1 and $2.5 \mathrm{GeV}$ is much less than what proposed in the KLOE-2 programme.

In the following subsections we present the main physics motivations for the off-peak running. We start with the improvements on the cross sections $\sigma\left(e^{+} e^{-} \rightarrow\right.$ hadrons $)$ in a wide center-of-mass energy range, from the $\pi \pi$ threshold up to $2.5 \mathrm{GeV}$ discussing the implications for precision tests of the SM (Sect. 5.1) and vector-meson spectroscopy (Sect. 5.5). The physics reach with the study of $\gamma \gamma$ processes is presented in Sect. 6.

\subsection{SM precision tests and $\sigma_{h a d}$ at low energy}

The comparison of the SM predictions with precision data served in the last few decades as an invaluable tool to test 
this theory at the quantum level. It has also provided stringent constraints on many NP scenarios. The remarkable agreement between the precision measurements of electroweak observables and their SM predictions is a striking experimental confirmation of the theory, even if there are a few observables where the agreement is not so satisfactory. On the other hand, the Higgs boson has not yet been observed and there are clear phenomenological facts (e.g., dark matter, matter-antimatter asymmetry in the universe) as well as strong theoretical arguments hinting at the presence of physics beyond the SM. Future colliders, such as the LHC or the ILC will hopefully answer many such questions offering at the same time, great physics potential, and a new challenge to provide even more precise theoretical predictions.

Precise SM predictions require precise input parameters. Among the three basic input parameters of the electroweak (EW) sector of the SM - the fine-structure constant $\alpha$, the Fermi coupling constant $G_{F}$ and the mass of the $Z$ boson $-\alpha$ is by far the most precisely known, determined mainly from the anomalous magnetic moment of the electron with an amazing relative precision of 0.37 parts per billion (ppb) 303. However, physics at nonzero squared momentum transfer $q^{2}$ is actually described by an effective electromagnetic coupling $\alpha\left(q^{2}\right)$ rather than by the low-energy constant $\alpha$ itself. The shift of the finestructure constant from the Thomson limit to high energy involves non-perturbative hadronic effects which spoil this fabulous precision. Indeed, the present accuracies of these basic parameters are 303, 18, 304, 305, 306

$$
\begin{gathered}
\frac{\delta \alpha}{\alpha} \sim 4 \times 10^{-10}, \frac{\delta \alpha\left(M_{Z}^{2}\right)}{\alpha\left(M_{Z}^{2}\right)} \sim \mathcal{O}\left(10^{-4}\right), \\
\frac{\delta M_{Z}}{M_{Z}} \sim 2 \times 10^{-5}, \frac{\delta G_{F}}{G_{F}} \sim 4 \times 10^{-6} .
\end{gathered}
$$

The relative uncertainty of $\alpha\left(M_{z}^{2}\right)$ is roughly one order of magnitude worse than that of $M_{Z}$, making it one of the limiting factors in the calculation of precise SM predictions. The effective fine-structure constant at the scale $M_{Z}$ $\alpha\left(M_{Z}^{2}\right)$ plays a crucial role in basic EW radiative corrections of the SM. An important example is the EW mixing parameter $\sin ^{2} \theta$, related to $\alpha, G_{F}$ and $M_{Z}$ via the Sirlin relation $307,308,309,310,311$

$$
\sin ^{2} \theta_{S} \cos ^{2} \theta_{S}=\frac{\pi \alpha}{\sqrt{2} G_{F} M_{Z}^{2}\left(1-\Delta r_{S}\right)}
$$

where the subscript $S$ identifies the renormalization scheme. $\Delta r_{S}$ incorporates the universal correction $\Delta \alpha\left(M_{z}^{2}\right)$, large contributions that depend quadratically on the top quark mass 312 (which led to its indirect determination before the discovery of this quark at the Tevatron 313,314]), plus all remaining quantum effects. Neglecting the latter sub-leading corrections, SM predictions of precision observables which have been measured at LEP would fail at the $10-\sigma$ level. In the SM, $\Delta r_{S}$ depends on various physical parameters such as $\alpha, G_{F}, M_{Z}, M_{W}, M_{H}, m_{f}$, etc., where $m_{f}$ stands for a generic fermion mass. As $M_{H}$, the mass of the Higgs boson, is the only relevant unknown parameter in the SM, important indirect bounds on this missing ingredient can be set by comparing the calculated quantity in Eq.(63) with the experimental value of $\sin ^{2} \theta_{S}$. These constraints can be easily derived using the simple formulae of Refs. 315, 316, 317, 318, 319, 320, which relate the effective EW mixing angle $\sin ^{2} \theta_{\text {eff }}^{\text {lept }}$ (measured at LEP and SLC from the on-resonance asymmetries) with $\Delta \alpha\left(M_{z}^{2}\right)$ and other experimental inputs such as the mass of the top quark. It is important to note that an error of $\delta \Delta \alpha\left(M_{Z}^{2}\right)=35 \times 10^{-5}$ 321 in the effective electromagnetic coupling constant dominates the uncertainty of the theoretical prediction of $\sin ^{2} \theta_{\text {eff }}^{\text {lept }}$, inducing an error $\delta\left(\sin ^{2} \theta_{\text {eff }}^{\text {lept }}\right) \sim 12 \times 10^{-5}$ (which is not much smaller than the experimental one $\delta\left(\sin ^{2} \theta_{\text {eff }}^{\text {lept }}\right)^{\mathrm{EXP}}=16 \times 10^{-5}$ determined by LEP and SLD 322,323) and affecting the Higgs boson mass bound. Moreover, as measurements of the effective EW mixing angle at a future linear collider may improve its precision by one order of magnitude 324, a much smaller value of $\delta \Delta \alpha\left(M_{Z}^{2}\right)$ will be required as discussed in the next subsection. It is therefore crucial to assess all viable options to further reduce this uncertainty. The latest global fit of the LEP Electroweak Working Group, which employs the complete set of EW observables, leads at present to the value $M_{H}=87_{-26}^{+35} \mathrm{GeV}$ and the $95 \%$ C.L. upper limit of $157 \mathrm{GeV}$ (Fig. 19] [323]. This limit increases to $186 \mathrm{GeV}$ when including the LEPII direct search lower limit of $114 \mathrm{GeV}$.

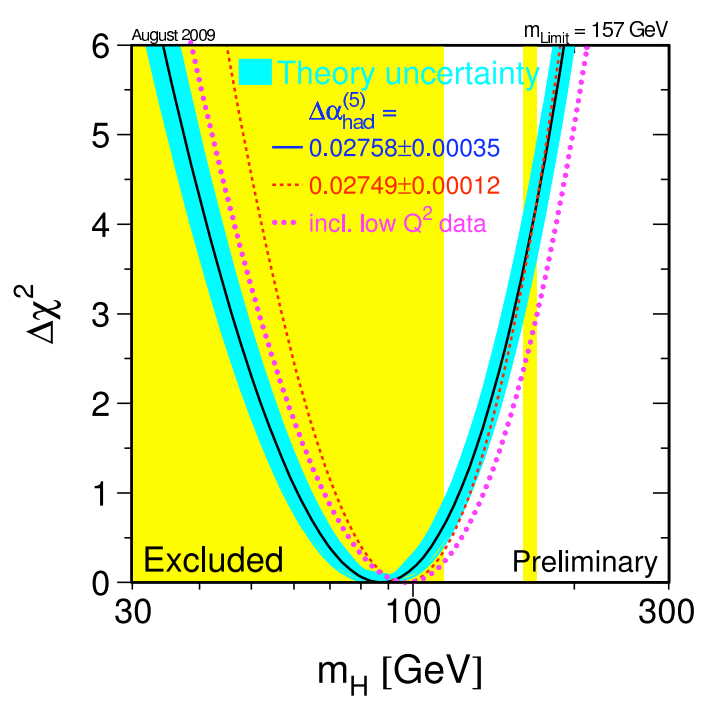

Fig. 19: The line is the result of the Electroweak Working Group fit using all data 323; the band represents an estimate of the theoretical error due to missing higher order corrections. The vertical bands show the $95 \%$ C.L. exclusion limits on $M_{H}$ from the direct searches. 


\subsection{The fine-structure constant at the $M_{Z}$ scale}

Let us examine the running of the effective fine-structure constant to the scale $M_{Z}$, that can be defined by $\Delta \alpha\left(M_{Z}^{2}\right)=$ $4 \pi \alpha \operatorname{Re}\left[\Pi_{\gamma \gamma}^{(f)}(0)-\Pi_{\gamma \gamma}^{(f)}\left(M_{z}^{2}\right)\right]$, where $\Pi_{\gamma \gamma}^{(f)}\left(q^{2}\right)$ is the fermionic part of the photon vacuum polarization function (with the top quark decoupled). Its evaluation includes hadronic contributions where long-distance QCD cannot be calculated analytically. These contributions cause the aforementioned dramatic loss of accuracy, by several orders of magnitude which occurs moving from the value of $\alpha$ at vanishing momentum transfer to that at $q^{2}=M_{z}^{2}$. The shift $\Delta \alpha\left(M_{Z}^{2}\right)$ can be divided in two parts: $\Delta \alpha\left(M_{Z}^{2}\right)=$ $\Delta \alpha_{\text {lep }}\left(M_{Z}^{2}\right)+\Delta \alpha_{\text {had }}^{(5)}\left(M_{z}^{2}\right)$. The leptonic contribution is calculable in perturbation theory and known up to three loops: $\Delta \alpha_{\text {lep }}\left(M_{z}^{2}\right)=3149.7686 \times 10^{-5}[325$. The hadronic contribution $\Delta \alpha_{\text {had }}^{(5)}\left(M_{z}^{2}\right)$ of the five quarks $(u, d, s, c$, and $b$ ) can be computed from hadronic $e^{+} e^{-}$annihilation data via the dispersion relation 326 :

$$
\Delta \alpha_{\text {had }}^{(5)}\left(M_{Z}^{2}\right)=-\left(\frac{\alpha M_{Z}^{2}}{3 \pi}\right) \operatorname{Re} \int_{4 m_{\pi}^{2}}^{\infty} d s \frac{R(s)}{s\left(s-M_{Z}^{2}-i \epsilon\right)},
$$

where $R(s)=\sigma^{(0)}(s) /\left(4 \pi \alpha^{2} / 3 s\right)$ and $\sigma^{(0)}(s)$ is the total cross section for $e^{+} e^{-}$annihilation into any hadronic state, with vacuum polarization and initial-state QED corrections subtracted off. In the 1990s, detailed evaluations of this dispersive integral have been carried out by several authors $327,328,329,330,331,332,333,334,335,336,337$. . recently, some of these analyses were updated to include new $e^{+} e^{-}$data - mostly from CMD-2 338, and BESII 339. - obtaining: $\Delta \alpha_{\text {had }}^{(5)}=2761(36) \times 10^{-5}$ [340], $\Delta \alpha_{\text {had }}^{(5)}=$ $2757(36) \times 10^{-5}[341], \Delta \alpha_{\text {had }}^{(5)}=2755(23) \times 10^{-5}$ [342], and $\Delta \alpha_{\text {had }}^{(5)}=2749(12) \times 10^{-5}[343$. The reduced uncertainty of the latter result has been obtained making stronger use of theoretical inputs. The reduction, by a factor of two, of the uncertainty quoted in Ref. 327. $\left(70 \times 10^{-5}\right)$ with respect to that in $341\left(36 \times 10^{-5}\right)$ is mainly due to the data from BESII. The latest updates, $\Delta \alpha_{\text {had }}^{(5)}=$ $2758(35) \times 10^{-5} 321, \Delta \alpha_{\text {had }}^{(5)}=2768(22) \times 10^{-5}$ 344, and $\Delta \alpha_{\text {had }}^{(5)}=2759.4(21.9) \times 10^{-5}$ 306, include also the KLOE measurements 345. In particular, the latest CMD2 346, 347,348 and SND 349 data where included in the derivations of Refs. 344, 306, and the BaBar 350, 351, 352 data were also used in that of Ref. 306. Table 14 from Ref. 341 shows that an uncertainty $\delta \Delta \alpha_{\text {had }}^{(5)} \sim 5 \times 10^{-5}$ needed for precision physics at a future linear collider requires the measurement of the hadronic cross section with a precision of $\mathcal{O}(1 \%)$ from $\pi \pi$ threshold up to the $\Upsilon$ peak.

As advocated in Ref. 353, the dispersion integral as in Eq. (64) can be calculated in a different way: it is sufficient to calculate $\Delta \alpha_{\text {had }}^{(5)}(s)$ not directly at $s=M_{Z}^{2}$, but at some much lower scale $s_{0}=-M_{0}^{2}$ in the Euclidean region, which is chosen such that the difference $\Delta \alpha_{\text {had }}^{(5)}\left(M_{Z}^{2}\right)-$ $\Delta \alpha_{\text {had }}^{(5)}\left(-M_{0}^{2}\right)$ can be reliably calculated using perturba-
Table 14: The uncertainties $\delta \Delta \alpha_{\text {had }}^{(5)}$ (first column) and the errors induced by these uncertainties on the theoretical SM prediction for $\sin ^{2} \theta_{\text {eff }}^{\text {lept }}$ (second column). The third column indicates the corresponding requirements on the $R$ measurement.

\begin{tabular}{|c|c|c|}
\hline$\delta \Delta \alpha_{\text {had }}^{(5)} \times 10^{5}$ & $\delta\left(\sin ^{2} \theta_{\text {eff }}^{\text {lept }}\right) \times 10^{5}$ & Request on $R$ \\
\hline \hline 22 & 7.9 & Present \\
\hline 7 & 2.5 & $\delta R / R \sim 1 \%$ up to $J / \psi$ \\
\hline 5 & 1.8 & $\delta R / R \sim 1 \%$ up to $\Upsilon$ \\
\hline
\end{tabular}

tive QCD (pQCD). In Eq. (64) pQCD is used to compute the high energy tail, including some perturbative windows at intermediate energies. An extended use of pQCD is possible by monitoring the validity of $\mathrm{pQCD}$ via the Adler function, essentially the derivative of $\Delta \alpha_{\text {had }}^{(5)}(s)$ evaluated in the spacelike region: $\frac{D\left(Q^{2}\right)}{Q^{2}}=-\left.\frac{3 \pi}{\alpha} \frac{d \Delta \alpha_{\mathrm{had}}}{d q^{2}}\right|_{q^{2}=-Q^{2}}$. Using a full-fledged state-of-the-art pQCD prediction for the Adler function one finds that $\Delta \alpha_{\text {had }}^{(5)}\left(-M_{Z}^{2}\right)-\Delta \alpha_{\text {had }}^{(5)}\left(-M_{0}^{2}\right)$ can be neatly calculated from the predicted Adler function 354 for $M_{0} \sim 2.5 \mathrm{GeV}$ as a conservative choice. Also the small missing $\Delta \alpha_{\text {had }}^{(5)}\left(M_{Z}^{2}\right)-\Delta \alpha_{\text {had }}^{(5)}\left(-M_{Z}^{2}\right)$ terms can safely be calculated in pQCD. The crucial point is that pQCD is used in a fully controlled manner, away from Mothesholds and resonances. There are three points to note: i) this strategy allows a more precise determination of $\Delta \alpha_{\text {had }}^{(5)}\left(M_{Z}^{2}\right)$ than the direct method based on Eq.(64); ii) it requires a precise QCD calculation and relies on a very precise determination of the QCD parameters $\alpha_{s}, m_{c}$ and $m_{b}$ 355; iii) the method relies mainly on the precise cross section measurements at low energy which at the same time are needed to reduce the uncertainty on the prediction of the muon $g-2$. Thus projects such as KLOE-2 are crucial for a better determination of the effective fine structure constant and the muon $g-2$ 306.

\subsection{The muon $g-2$}

During the last few years, in a sequence of measurements of increasing precision, the E821 collaboration at the BNL has determined $a_{\mu}=\left(g_{\mu}-2\right) / 2$ with a fabulous relative precision of 0.5 parts per million (ppm) 356, 357. 358 359, 360, allowing us to test all sectors of the SM and to scrutinize viable alternatives to this theory 361 , 362363 . The present world average experimental value is $a_{\mu}^{\text {EXP }}=116592089(63) \times 10^{-11}(0.54 \mathrm{ppm})$ 359, 360, 364. This impressive result is still limited by statistical errors, and a proposal to measure the muon $g-2$ to a precision of $0.14 \mathrm{ppm}$ has recently been submitted to FNAL 365 . But how precise is the theoretical prediction?

The SM prediction of the muon $g-2$ is conveniently divided into QED, electroweak (EW) and hadronic (leadingand higher-order) contributions: $a_{\mu}^{\mathrm{SM}}=a_{\mu}^{\mathrm{QED}}+a_{\mu}^{\mathrm{EW}}+a_{\mu}^{\mathrm{HLO}}+$ 
$a_{\mu}^{\mathrm{HHO}}$. The QED prediction, computed up to four (and estimated at five) loops, currently stands at

$a_{\mu}^{\mathrm{QED}}=116584718.10(16) \times 10^{-11} 366,367,368,369,370$ 371, 372, 373, while the EW effects, suppressed by a factor $\left(m_{\mu}^{2} / M_{W}^{2}\right)$, provide $a_{\mu}^{\mathrm{EW}}=154(2) \times 10^{-11}$ 374, 375, 376.

As in the case of the effective fine-structure constant at the scale $M_{Z}$, the SM determination of the anomalous magnetic moment of the muon is presently limited by the evaluation of the hadronic vacuum polarization and, in turn, by our knowledge of the low-energy total cross section for $e^{+} e^{-}$annihilation into hadrons. Indeed, the hadronic leading-order contribution $a_{\mu}^{\mathrm{HLO}}$, due to the hadronic vacuum polarization correction to the one-loop diagram, involves long-distance QCD effects which cannot be computed perturbatively. However, using analyticity and unitarity, it was shown long ago that this term can be computed from hadronic $e^{+} e^{-}$annihilation data via the dispersion integral 377

$$
\begin{aligned}
a_{\mu}^{\mathrm{HLO}} & =\left(1 / 4 \pi^{3}\right) \int_{4 m_{\pi}^{2}}^{\infty} d s K(s) \sigma^{(0)}(s) \\
& =\left(\alpha^{2} / 3 \pi^{2}\right) \int_{4 m_{\pi}^{2}}^{\infty} d s K(s) R(s) / s,
\end{aligned}
$$

where $K(s)$ is a kernel function which decreases monotonically for increasing $s$. This integral is similar to the one entering the evaluation of the hadronic contribution $\Delta \alpha_{\text {had }}^{(5)}\left(M_{Z}^{2}\right)$ in Eq.(64). Here, however, the weight function in the integrand gives a stronger weight to low-energy data. Figure 20 from Ref. 378] shows the fractions of the total contributions and (errors) ${ }^{2}$ from various energy intervals in the dispersion integrals for $a_{\mu}^{\mathrm{HLO}}$ and $\Delta \alpha_{\text {had }}^{(5)}\left(M_{Z}^{2}\right)$. An important role among all $e^{+} e^{-}$annihilation measurements is played by the precise data collected by the CMD2 338,346, 347,348 and SND 349 experiments at the VEPP-2M collider in Novosibirsk for the $e^{+} e^{-} \rightarrow \pi^{+} \pi^{-}$ cross section in the energy ranges $\sqrt{s} \in[0.37,1.39] \mathrm{GeV}$ and $\sqrt{s} \in[0.39,0.98] \mathrm{GeV}$, respectively. In 2004 the KLOE experiment has obtained a precise measurement of $\sigma\left(e^{+} e^{-}\right.$ $\left.\pi^{+} \pi^{-}\right)$via the initial-state radiation (ISR) method at the $\phi$ resonance 345 . This cross section was extracted for $\sqrt{s}$ between 0.59 and $0.97 \mathrm{GeV}$ with a systematic error of $1.3 \%$ and a negligible statistical one. More recently KLOE published a new measurement with larger statistics and a systematic error of $0.9 \% 379$. KLOE, CDM-2 and SND give consistent contributions to $a_{\mu}$, and the evaluations of the dispersive integral in Eq. 65 are in good agreement: $a_{\mu}^{\mathrm{HLO}}=6894(42)_{\mathrm{exp}}(18)_{\mathrm{rad}} \times 10^{-11} 344, a_{\mu}^{\mathrm{HLO}}=$ $6909(39)_{\exp }(20)_{\mathrm{th}} \times 10^{-11} 380,381, a_{\mu}^{\mathrm{HLO}}=6903.0(52.6) \times$ $10^{-11} 382,383$.

The $e^{+} e^{-} \rightarrow \pi^{+} \pi^{-}(\gamma)$ process via ISR has also been studied by BaBar, which has recently reported a new measurement of the $\pi^{+} \pi^{-}(\gamma)$ cross section from threshold up to $3 \mathrm{GeV}$ 384. The BaBar data have been included in a new evaluation of the lowest order hadronic contribution to $a_{\mu}: a_{\mu}^{\mathrm{HLO}}=6955.0(41.0) \times 10^{-11} 385$.

The term $a_{\mu}^{\mathrm{HLO}}$ can alternatively be computed incorporating hadronic $\tau$-decay data, related to those of hadroproduction in $e^{+} e^{-}$collisions via isospin symmetry [337, 386,

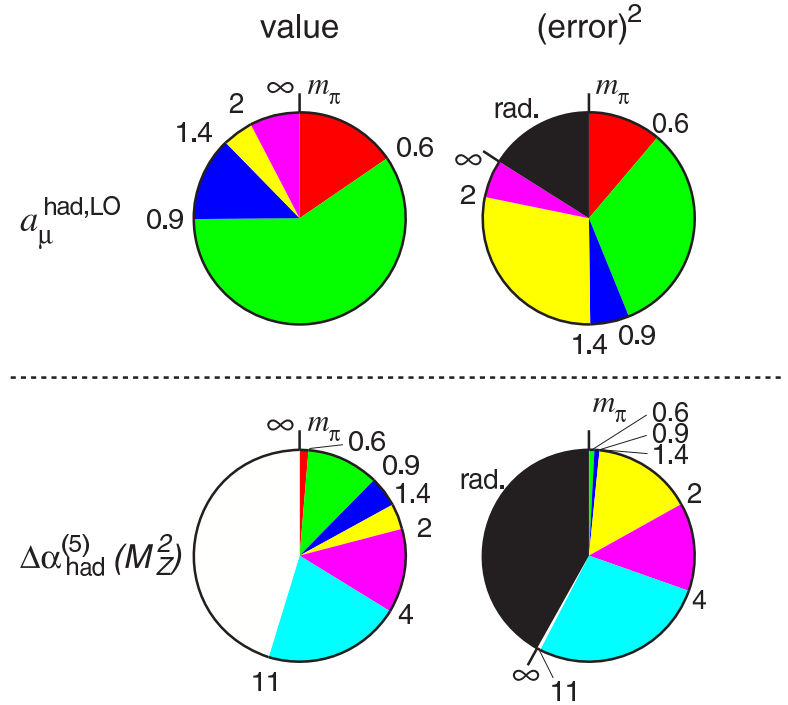

Fig. 20: The pie diagrams show the fractions of the total contributions and (errors) ${ }^{2}$ from various energy intervals in the dispersion integrals in Eqs. (64), 650. The diagrams for the LO hadronic contribution to $g-2$, shown in the first row, correspond to sub-contributions with energy boundaries at $0.6,0.9,1.4,2 \mathrm{GeV}$ and $\infty$, whereas for the hadronic contribution to the effective fine-structure constant, shown in the second row, the boundaries are at 0.6, $0.9,1.4,2,4,11.09 \mathrm{GeV}$ and $\infty$. In the (error) ${ }^{2}$ diagrams, the contributions arising from the treatment of the radiative corrections to the data are also included [378.

387. Unfortunately, even if isospin violation corrections are taken into account $388,389,390,391$, the $\tau$-based value is higher than the $e^{+} e^{-}$-based one, leading to a small $(\sim 2$ $\sigma) \Delta a_{\mu}$ difference 392 . As the $e^{+} e^{-}$data are more directly related to the $a_{\mu}^{\mathrm{HLO}}$ calculation than the $\tau$ ones, the latest analyses do not include the latter. Also, recently studied additional isospin-breaking corrections somewhat reduce the difference between these two sets of data (lowering the $\tau$-based determination) [393, 394, 392, and a new analysis of the pion form factor claims that the $\tau$ and $e^{+} e^{-}$ data are consistent after isospin violation effects and vector meson mixing are considered 395, 396, 397.

The higher-order hadronic term is further divided into two parts: $a_{\mu}^{\mathrm{HHO}}=a_{\mu}^{\mathrm{HHO}}(\mathrm{vp})+a_{\mu}^{\mathrm{HHO}}(\mathrm{lbl})$. The first one, $-98(1) \times 10^{-11} 344$, is the $\mathcal{O}\left(\alpha^{3}\right)$ contribution of diagrams containing hadronic vacuum polarization insertions [398]. The second term, also of $\mathcal{O}\left(\alpha^{3}\right)$, is the hadronic lightby-light contribution; as it cannot be determined from data, its evaluation relies on specific models. Three major components of $a_{\mu}^{\mathrm{HHO}}(\mathrm{lbl})$ can be identified: charged-pion loops, quark loops, and pseudoscalar $\left(\pi^{0}, \eta\right.$, and $\left.\eta^{\prime}\right)$ pole diagrams. The latter ones dominate the final result and require information on the electromagnetic form factors of the pseudoscalars (c.f. Sect. 4.2 and Sect. 6.2.2). Recent determinations of $a_{\mu}^{\mathrm{HHO}}(\mathrm{lbl})$ vary between $80(40) \times$ $10^{-11}$ [399, 400] and $136(25) \times 10^{-11}$,401. The latest ones, $105(26) \times 10^{-11} 402$ and $116(39) \times 10^{-11} 403,383$, lie be- 
tween them. If we add the second of these two results to the leading-order hadronic contribution, for example the value of Refs. 382 383, and the rest of the SM contributions, we obtain $a_{\mu}^{\mathrm{SM}}=116591793(66) \times 10^{-11}$. The difference with the experimental value is then $\Delta a_{\mu}=a_{\mu}^{\mathrm{EXP}}-a_{\mu}^{\mathrm{SM}}$ $=296(91) \times 10^{-11}$, i.e., 3.3 standard deviations (all errors were added in quadrature). Slightly higher discrepancies are obtained employing the values of the leadingorder hadronic contribution reported in Refs. 380,381 or 344. Recent reviews of the muon $g-2$ can be found in Refs. 373 382, 383, 404, 405 406 407, 408 409|410.

Hypothetical errors in the SM prediction that could explain the present discrepancy with the experimental value were discussed in Ref. 411,412. The authors concluded that none of them looks likely. In particular, a hypothetical increase of the hadroproduction cross section in lowenergy $e^{+} e^{-}$collisions could bridge the muon $g-2$ discrepancy, but it was shown to be unlikely in view of current experimental error estimates. If, nonetheless, this turns out to be the explanation of the discrepancy, it was shown that the $95 \%$ C.L. upper bound on the Higgs boson mass is then reduced to about $130 \mathrm{GeV}$ which, in conjunction with the experimental $114.4 \mathrm{GeV} 95 \%$ C.L. lower bound, leaves a narrow window for the mass of this fundamental particle.

The analysis of this section shows that while the QED and EW contributions to the anomalous magnetic moment of the muon appear to be ready to rival the forecasted precisions of future experiments, much effort will be needed to reduce the hadronic uncertainty. This effort is challenging but possible, and certainly well motivated by the excellent opportunity the muon $g-2$ is providing us to unveil (or constrain) NP effects. Once again, a medium-term program of hadronic cross section measurements is clearly essential.

\section{$5.4 \sigma_{h a d}$ measurements at low energy}

In the last years big efforts on $e^{+} e^{-}$data in the energy range below a few $\mathrm{GeV}$ led to a substantial reduction in the hadronic uncertainty on $\Delta \alpha^{\text {had }}$ and $a_{\mu}^{\text {had }}$. Figure 21 shows an up-to-date compilation of this data. The main improvements have been achieved in the region below 5 $\mathrm{GeV}$ : between 2 and $5 \mathrm{GeV}$ (where the data are now closer to the prediction of pQCD), the BESII collaboration reduced the error to $\sim 7 \% 339$ (before it was $\sim 15 \%$ ); between 1 and $4.5 \mathrm{GeV}$ BaBar measured various final states with more than two hadrons with a systematic accuracy between $5 \%$ and $15 \%$, as shown in Tab. 15 below $1 \mathrm{GeV}$, the CMD-2 and SND collaborations at Novosibirsk, KLOE at Frascati and BaBar at Stanford measured the pion form factor in the energy range around the $\rho$ peak with a systematic error of $0.8 \%, 1.3 \%, 0.9 \%$, and $0.5 \%$, respectively. The CMD-2 and SND collaborations at Novosibirsk and BESII in Beijing were performing the hadronic cross section measurements in a traditional way, i.e., by varying the $e^{+} e^{-}$beam energies. KLOE, BaBar, and more recently Belle used the method of radiative return 418, 419, 420, 421, 422, 423, 424, 425, as reviewed in Refs. 426, 427.

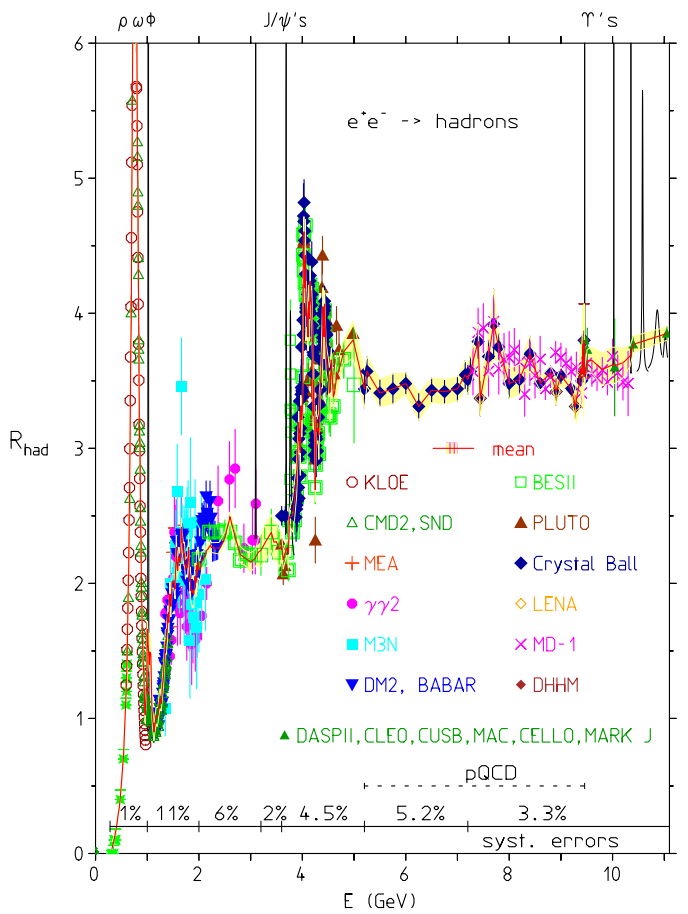

Fig. 21: An updated compilation of $R$ measurements. In the bottom line the overall uncertainties of the different regions are reported.

Table 15: Systematic accuracy on several processes studied by BaBar in the energy range $\sqrt{s}<4.5 \mathrm{GeV}$ using ISR. Integrated luminosity is $232 \mathrm{fb}^{-1}$ for all processes but $3 \pi$ and $2 \pi^{+} 2 \pi^{-}$where only $89 \mathrm{fb}^{-1}$ were used.

\begin{tabular}{|c|c|r|}
\hline Process & Systematic accuracy & Reference \\
\hline \hline$\pi^{+} \pi^{-} \pi^{0}$ & $(6-8) \%$ & 413 \\
$2 \pi^{+} 2 \pi^{-}$ & $5 \%$ & 414 \\
$2 \pi 2 \pi^{0}$ & $(8-14) \%$ & 415 \\
$2 \pi^{+} 2 \pi^{-} \pi^{0}$ & $(8-11) \%$ & 351 \\
$2 \pi^{+} 2 \pi^{-} \eta$ & $7 \%$ & 351 \\
$3 \pi^{+} 3 \pi^{-}+2 \pi^{+} 2 \pi^{-} 2 \pi^{0}$ & $(6-11) \%$ & 416 \\
$K K \pi$ & $(5-6) \%$ & 417 \\
$K^{+} K^{-} \pi \pi$ & $(8-11) \%$ & 350 \\
\hline
\end{tabular}

Figure 21 shows that, despite the recent progress, the region between 1 and $2 \mathrm{GeV}$ is still poorly known, with a fractional accuracy of $\sim 10 \%$. Since about $50 \%$ of the error squared, $\delta^{2} a_{\mu}^{\text {had }}$, comes from this region (and about $70 \%$ of $\left.\delta^{2} \Delta \alpha_{\text {had }}^{(5)}\left(-M_{0}^{2}\right)\right)$, it is evident how desirable an improvement on this region is.

\subsection{Vector-meson spectroscopy}

Cross sections of exclusive final states are also important for the spectroscopy of vector mesons whose properties provide fundamental information on interactions of 
light quarks. The $\omega(1420), \rho(1450), \omega(1650), \phi(1680)$, and $\rho(1700)$ have been found between 1 and $2 \mathrm{GeV}$ [18] but even their basic parameters $\left(M, \Gamma, \Gamma_{e e}\right)$ are poorly known. The mass of these states is in satisfactory agreement with QCD which actually predicts three sets of vectors $\rho, \omega, \phi$ from 1 to $2 \mathrm{GeV}$ 428]. Recent studies of $e^{+} e^{-} \rightarrow \pi^{+} \pi^{-} \pi^{0}$ by SND 429 and BaBar 413 as well as $e^{+} e^{-} \rightarrow 2 \pi^{+} 2 \pi^{-} \pi^{0}$ by CMD-2 430 and BaBar 351, have significantly affected the $\omega(1420)$ and $\omega(1650)$ parameters. In the $\pi^{+} \pi^{-} \pi^{0}$ final state the cross sections measured by SND and BaBar are in good agreement at $\sqrt{s}<1.4 \mathrm{GeV}$, whereas above this energy the BaBar cross section is more than two times larger than what previously measured by DM2 431. The estimated values of the leptonic width of the $\omega(1420)$ and $\omega(1650)$ states are significantly higher than what found in Ref. 428. The $\rho(1450)$ and $\rho(1700)$ are expected to predominantly decay into four pions. Various measurements of both $2 \pi^{+} 2 \pi^{-}$and $\pi^{+} \pi^{-} 2 \pi^{0}$ agree with each other [432,433,434,414] showing one broad structure only, while three interfering $\rho$ 's have been observed in the twopion decay of the $\tau$-lepton 435 . A peculiar interference pattern or the existence of additional exotic states close to the regular $q \bar{q}$ states and mixed with them 436,437 can explain these results. It is also possible that the $\rho(1450)$ and $\rho(1700)$ have different decay modes. BaBar has recently reported the observation of two structures at $\sim 1.5 \mathrm{G}$ The broad one in the $K K^{*}$ state (width of $418 \pm 25 \pm$ $4 \mathrm{MeV}$ ) could be interpreted as the overlapping of $\rho(1450)$ and $\rho(1700)$ mesons, but the one in the $\phi \pi^{0}$ final state with a smaller width of $144 \pm 75 \pm 43 \mathrm{MeV}$, should have a different origin. Its properties are very close to those of the $C(1480)$ observed more than 20 years ago in $\pi^{-} p$ collisions 438. The $\phi \pi^{0}$ cross section also exhibits some narrow structures at $1.9 \mathrm{GeV}$, which could be the $\rho(1900)$ earlier observed in the $3 \pi^{+} 3 \pi^{-}$state photoproduction 439 , in the total $R$ at $e^{+} e^{-}$collider 440 as well as in both $3 \pi^{+} 3 \pi^{-}$and $2 \pi^{+} 2 \pi^{-} 2 \pi^{0}$ cross sections measured by DM2 and BaBar 416 (although with a larger width). The statistical significance of these findings is not sufficient to exclude that an OZI-violating decay mode of the $\rho(1700)$ was observed 416. BaBar also reports clear evidence for the $\rho(2150)$ meson in the $\eta^{\prime}(958) \pi^{+} \pi^{-}$and $f_{1}(1285) \pi^{+} \pi^{-}$ cross sections [351. Finally, BESII has recently reported evidence for a very broad $(\sim 800 \mathrm{MeV}) K^{+} K^{-}$state at about $1.5 \mathrm{GeV}$ produced in $J / \psi$ decays 442 , with isovector quantum numbers which have been obtained from a partial-wave analysis. These measurements can hardly be reconciled with what was observed to both $K^{+} K^{-}$and $K_{S}^{0} K_{L}^{0}$ final states.

Evidence for the $\phi(1680)$ was only based on an old observation of the structure in the $K_{S}^{0} K^{ \pm} \pi^{\mp}$ final state by DM1 443 . BaBar has recently confirmed the resonance in both $K K^{*}$ and $\phi \eta$ 417 modes while Belle has found it in the $\phi \pi^{+} \pi^{-}$final state 444. There is, however, a conflicting result of FOCUS [45], which observes a structure with similar parameters in the $K^{+} K^{-}$and does not observe it in the $K K^{*}$ channel.

BaBar has observed a new isoscalar resonance in the $K^{+} K^{-} \pi^{+} \pi^{-}$and $K^{+} K^{-} \pi^{0} \pi^{0}$ final states (predominantly $\left.\phi f_{0}(980)\right)$ at $2.12 \mathrm{GeV}$ 414,350. This result is confirmed by BESII with the analysis of the same final state in $J / \psi \rightarrow \eta \phi f_{0}(980)$ decay 446 and by Belle 444.

There are still many puzzles and unknown things calling for more theoretical and experimental efforts. Some progress, limited by the number of events, is expected from the ISR studies at BaBar and Belle. A real breakthrough can happen with the direct scanning at lower-energy colliders, VEPP-2000 and DA $\Phi$ NE.

Before discussing the improvements on the hadronic cross section measurements expected from KLOE-2 in the region $\left[2 m_{\pi}-2.5 \mathrm{GeV}\right]$, we review the status of the main competitor, VEPP-2000.

\subsection{VEPP-2000 prospects}

The VEPP-2000 $e^{+} e^{-}$collider at the Budker Institute of Nuclear Physics (BINP) in Novosibirsk will cover the energy range up to $2 \mathrm{GeV}$ which accounts for the $~ 92 \%$ of the hadronic contribution to the $g-2$ of the muon. Two different detectors will take data at VEPP-2000: the upgraded Spherical Neutral Detector (SND) and newly constructed Cryogenic Magnetic Detector (CMD-3). SND is a non-magnetic detector, whose basic part is a three-layer

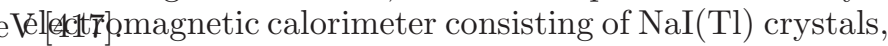
13.4 radiation-length long. The calorimeter covers nearly $90 \%$ of the full solid angle. Charged-particle tracking is provided by a cylindrical drift chamber. Particle identification is performed with aerogel Cherenkov counters of high density $(n=1.13)$.

CMD-3 is a magnetic detector based on a cylindrical drift chamber (DC) to measure coordinates, angles and momenta of charged particles. The DC is surrounded by a Z-chamber $(\mathrm{ZC})$ to measure the coordinate along the beam direction. DC and $\mathrm{ZC}$ are placed inside the thin $(\sim$ 4[18 $18 X_{0}$ ) superconducting solenoid with a magnetic field of $1.3 \mathrm{~T}$. Coordinates and energies of photons are measured by the barrel system based on the liquid Xe calorimeter $\left(7 X_{0}\right)$ and CsI $\left(8 X_{0}\right)$ crystals. BGO crystals $\left(13 X_{0}\right)$ are used in the end cap calorimeter.

As a rule, the pion form factor is calculated via the ratio $N_{\pi \pi} / N_{e e}$ which is directly measured using collinear events. The trigger and reconstruction inefficiencies cancel out partly in this ratio. Geometrical efficiencies $\epsilon_{e e}$ and $\epsilon_{\pi \pi}$ are calculated via Monte-Carlo simulation together with corrections for the detector imperfections. To achieve a systematic error less than $0.1 \%$ the beam energy must be measured with $10^{-4}$ fractional uncertainty or better. The variation of the derivative with the energy, $d\left|F_{\pi}(\sqrt{s})\right|^{2} / d \sqrt{s} /\left|F_{\pi}(\sqrt{s})\right|^{2} \cdot \Delta \sqrt{s}\left(\Delta E=10^{-3} E\right)$ does not exceed $\pm 1 \%$ but in the energy range near $\omega$ and $\phi$ mesons where it is about $6 \%$.

Radiative corrections are included in the Monte Carlo generator (MCGPJ) by means of the Structure Function approach. The estimated theoretical error on the process $e^{+} e^{-} \rightarrow \pi^{+} \pi^{-}, K^{+} K^{-}$(with point-like pions and kaons) is $0.2 \%$. A similar (or even better) accuracy is expected for channels with neutral particles in the final state because only ISR contributes to the cross sections. 
In a few years CMD-3 and SND experiments at VEPP2000 will provide new precision results on the measurements of the exclusive hadronic cross sections below 2 $\mathrm{GeV}$. Progress is particularly expected in the $e^{+} e^{-} \rightarrow$ $\pi^{+} \pi^{-}$channel, where a systematic uncertainty of about $0.4 \%$ or even better will be achieved.

\subsection{Improving $\sigma_{\pi \pi}$ below $1 \mathrm{GeV}$ with KLOE-2}

The region below $1 \mathrm{GeV}$ is dominated by the two-pion channel which accounts for $70 \%$ of the contribution to $a_{\mu}^{\text {had }}$, and for $40 \%$ to the total error $\delta^{2} a_{\mu}$ as shown in Fig. 20. How can this error be reduced? Let us consider the region around the $\rho$ and $\pi^{+} \pi^{-}$threshold: i) the $\pi^{+} \pi^{-}$ region between 0.5 and $1 \mathrm{GeV}$ has been studied by different experiments. CMD-2 and SND have performed an energy scan at the $e^{+} e^{-}$collider VEPP-2M $(\sqrt{s} \in[0.4-$ 1.4] $\mathrm{GeV}$ ) with $\sim 10^{6}$ and $\sim 4.5 \times 10^{6}$ events respectively, and systematic fractional errors from $0.6 \%$ to $4 \%$ in the cross sections, depending on $\sqrt{s}$. The pion form factor has also been measured by KLOE using ISR, and more recently by BaBar. KLOE collected more than 3.1 million events, corresponding to an integrated luminosity of 240 $\mathrm{pb}^{-1}$, leading to a relative error of $0.9 \%$ in the energy region [0.6-0.97] GeV dominated by systematics. BaBar has presented a $\pi^{+} \pi^{-}(\gamma)$ cross section measurement based on half a million selected events. The pion form factor is obtained by the ratio $\pi^{+} \pi^{-}(\gamma)$ to $\mu^{+} \mu^{-}(\gamma)$ which allows a systematic error of $0.5 \%$ in the $\rho$ region increasing to $1 \%$ outside; ii) the threshold region $\left[2 m_{\pi}-0.5 \mathrm{GeV}\right]$ provides $13 \%$ of the total $\pi^{+} \pi^{-}$contribution to the muon anomaly: $a_{\mu}^{\mathrm{HLO}}\left[2 m_{\pi}-0.5 \mathrm{GeV}\right]=(58.0 \pm 2.1) \times 10^{-10}$. To overcome the lack of precision data at threshold energies, the pion form factor is extracted from a parametrization based on ChPT, constrained from spacelike data 447. The most effective way to measure the cross section near the threshold in the timelike region is provided by ISR events, where the emission of an energetic photon allows the study of the two pions at rest. However at DA $\Phi$ NE, the process $\phi \rightarrow \pi^{+} \pi^{-} \pi^{0}$ with one missing photon is hundreds of times more frequent than the signal, and therefore a precision measurement requires an accurate evaluation of the background. Furthermore, irreducible background due to $\phi \rightarrow \pi^{+} \pi^{-} \gamma$ is also present while running at the $\phi$ - resonance peak. The background issue can be largely overcome by taking data at $\sqrt{s}<M_{\phi}$ : KLOE has analysed about $200 \mathrm{pb}^{-1}$ of integrated luminosity at $1 \mathrm{GeV}$ and a new measurement of the pion form factor has been recently presented 448 .

Figure22 shows the statistical precision that can be reached in the region below $1 \mathrm{GeV}$ with an integrated luminosity of $2 \mathrm{fb}^{-1}$ at $1 \mathrm{GeV}$ for each bin of $\Delta s=0.01 \mathrm{GeV}^{2}$. This luminosity leads to a statistical error on $a_{\mu}^{\mathrm{HLO}}$ of a few per mill. The experimental systematic error could be kept at the same level (now is at $1 \%$ ) by taking data at $1 \mathrm{GeV}$, where the background conditions (mainly from $\phi \rightarrow \pi^{+} \pi^{-} \gamma$ and $\phi \rightarrow \pi^{+} \pi^{-} \pi^{0}$ ) which especially affect the threshold region, are much more favourable. The theoretical error, of the order of $0.6 \%$, dominated by the uncertainty on the

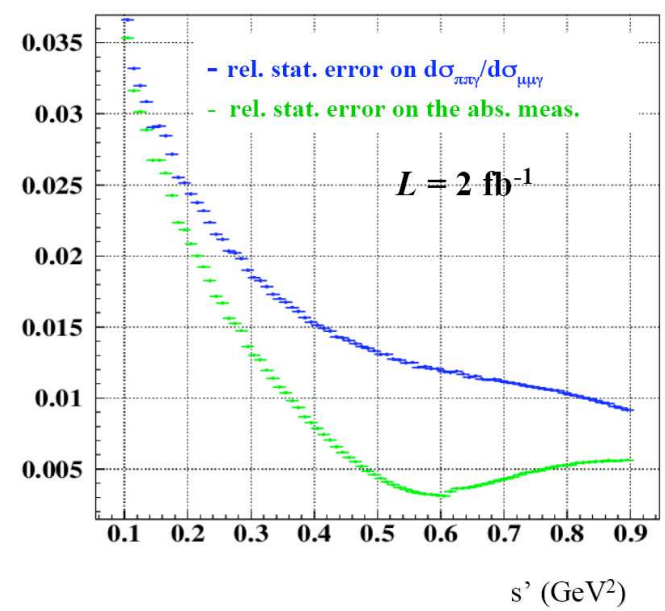

Fig. 22: The fractional statistical error on the cross section $\mathrm{d} \sigma_{\pi \pi \gamma} / \mathrm{d} M_{\pi \pi}^{2}$ and on the ratio $\mathrm{d} \sigma_{\pi \pi \gamma} / \mathrm{d} \sigma_{\mu \mu \gamma}$ as a function of the $\pi \pi$ invariant mass squared, $s^{\prime}=M_{\pi \pi}^{2}$ (bin width $=0.01 \mathrm{GeV}^{2}$ ) for an integrated luminosity of $2 \mathrm{fb}^{-1}$ at 1 $\mathrm{GeV}$.

radiator function $(=0.5 \%)$, could be reduced to a few per mill in the future. The dependence on the theory is much reduced by the measurement of the $\pi \pi \gamma / \mu \mu \gamma$ ratio where the main uncertainty on the radiator function and vacuum polarization cancel out in the ratio so that with an integrated luminosity of $2 \mathrm{fb}^{-1}$ at $1 \mathrm{GeV}$ a fractional error of $0.4 \%$ could be reached.

\subsection{Improving $\sigma_{h a d}$ above $1.02 \mathrm{GeV}$ with KLOE-2}

The region [1-2.5] GeV, with an uncertainty on $\sigma_{\text {had }}$ of roughly $11 \%$, is the most poorly known, and contributes about $55 \%$ of the uncertainty on $a_{\mu}^{\mathrm{HLO}}$ and $40 \%$ of the error on $\Delta \alpha_{h a d}^{(5)}\left(M_{Z}^{2}\right)$ 306. In this region BaBar has published results on $e^{+} e^{-}$into three and four hadrons, obtained with an integrated luminosity of $89 \mathrm{fb}^{-1}$ [13. KLOE-2 can improve both the exclusive and inclusive measurements. At $10^{32} \mathrm{~cm}^{-2} \mathrm{~s}^{-1}$ luminosity, a scan in the region from 1 to $2.5 \mathrm{GeV}$ aiming at an integrated luminosity of $20 \mathrm{pb}^{-1}$ per point corresponds to few days of data taking for each energy bin. By assuming an energy step of $25 \mathrm{MeV}$, the whole region would be thus scanned in one year of data taking. As shown in Fig. 23, the statistical yield would be one order of magnitude higher than what achieved with 1 $a b^{-1}$ at BaBar, and better than what expected at BESIII with $10 \mathrm{fb}^{-1}$ at $3 \mathrm{GeV}$. Figure 24 shows the statistical error on the $\pi^{+} \pi^{-} \pi^{0}, 2 \pi^{+} 2 \pi^{-}$and $\pi^{+} \pi^{-} K^{+} K^{-}$channels which can be achieved by an energy scan of $20 \mathrm{pb}^{-1}$ per point, compared with Babar published $\left(89 \mathrm{fb}^{-1}\right)$ and tenfold $\left(890 \mathrm{fb}^{-1}\right)$ statistics. The energy scan allows a statistical accuracy at $1 \%$ level for most of the energy points. In addition, KLOE- 2 can benefit of the high machine luminosity and use ISR as well. 


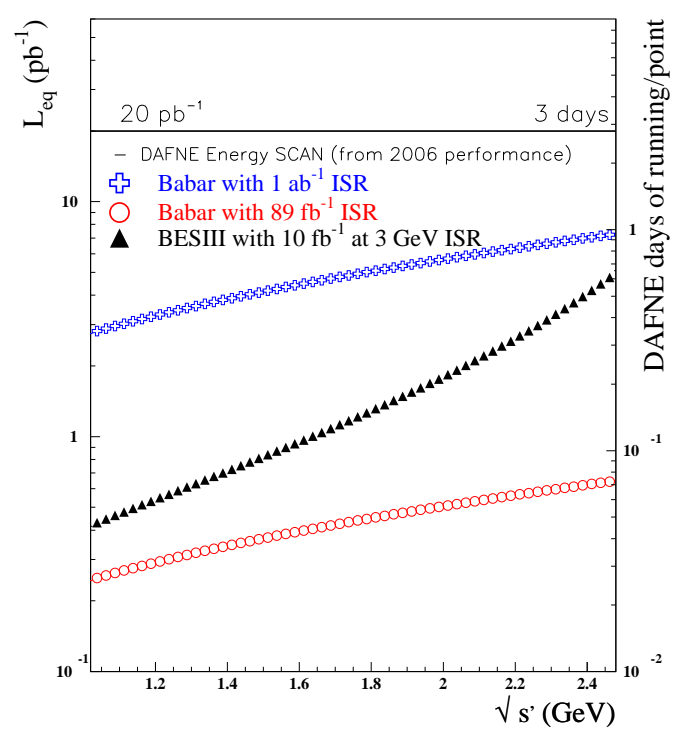

Fig. 23: Comparison of the event yield in terms of "equivalent" luminosity for BaBar with $89 \mathrm{fb}^{-1}$ (circle); BaBar with $1 \mathrm{ab}^{-1}$ (cross); BESIII using $10 \mathrm{fb}^{-1}$ at $3 \mathrm{GeV}$ (triangle). A polar angle of the photon larger than $20^{\circ}$ and a bin width of $25 \mathrm{MeV}$ have been assumed.
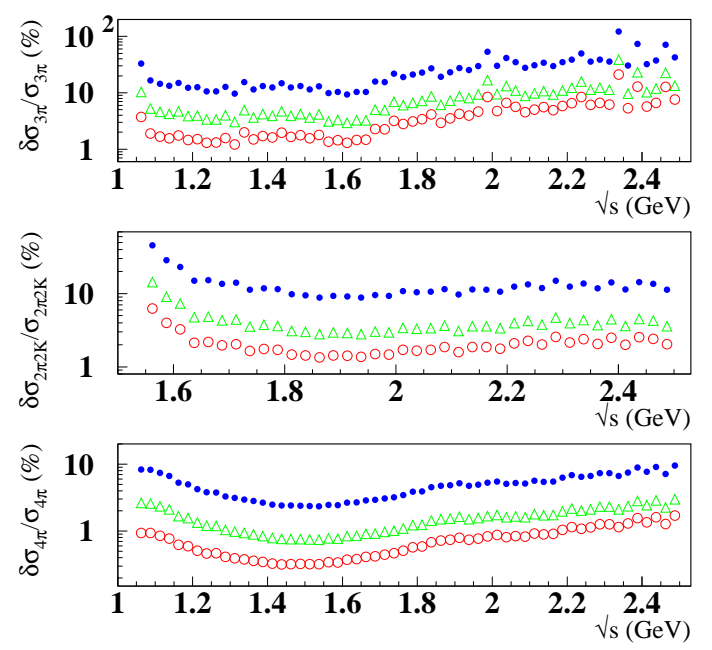

Fig. 24: Comparison of the statistical accuracy in the cross-section among an energy scan with $20 \mathrm{pb}^{-1}$ per point (०) at KLOE-2; the published BaBar results (•), BaBar with $890 \mathrm{pb}^{-1}$ of integrated luminosity (triangle) for the $\pi^{+} \pi^{-} \pi^{0}$ (top), $\pi^{+} \pi^{-} K^{+} K^{-}$(middle) and $2 \pi^{+} 2 \pi^{-}$(bottom) channels. An energy step of $25 \mathrm{MeV}$ is assumed.

The energy scan for a precision measurement of the cross sections requires the knowledge of the energy of the beams circulating in the collider with a fractional accuracy of $\mathcal{O}\left(10^{-4}\right)$, i.e., $\sim 100 \mathrm{keV}$ at $1 \mathrm{GeV}$. Two well estabilished techniques exist: the resonant depolarization (RD) technique and Compton backscattering (CBS) of laser photons against the electron beam, as reviewed in Ref. 449. The RD technique provides an accuracy of $1-3 \mathrm{keV}$ and is based on polarized electrons, a requirement not easy to fullfil at the low-energy colliders and making in addition the energy determination a very time-consuming task. The CBS method is less accurate, but does not need of any special condition on the beams. For this reason, it seems more suitable for DA $\Phi N E$. The measurement procedure consists of the acquisition of the order of $10^{6}$ events providing an accurate determination of the photon Compton spectrum. In one acquisition hour at VEPP-4M 449 a statistical accuracy on the beam energy of $70-100 \mathrm{keV}$ has been obtained with the CBS method. The simultaneous measurement of the energy of the scattered electron can be used to improve the precision on the beam energy. This is what has been done at the GRAAL beam at the ESRF machine in Grenoble. The GRAAL beam is a Compton-backscattered beam with maximal photon energy of $1.5 \mathrm{GeV}\left(\omega_{l}=3.51\right.$ $\left.\mathrm{eV}, E_{e}=6 \mathrm{GeV}\right)$, mainly devoted to photonuclear reactions 450. At GRAAL the determination of the $\gamma$-ray energy is achieved by tagging the scattered electrons which are separated from the primary beam by machine optics (bending magnets and quadrupoles) downstream to the laser-beam interaction region. The tagging detector consists of a silicon $\mu$ strip placed in a suitable position along the machine lattice, and measuring the displacement of these electrons from the orbit of nominal-energy particles. From the displacement, the energy of the electron is inferred and thus the photon one. The advantage of this approach lies in the great accuracy of the position measurements of the silicon strip detectors. A precision in the energy beam to the $10 \mathrm{keV}$ level was actually achieved with the $\mu$ strip detectors 451.

\subsection{Summary on $\sigma_{h a d}$ measurements}

In summary, precision tests of the SM require a more accurate knowledge of the hadronic cross section in the whole energy range, from the $2 m_{\pi}$ threshold to $2.5 \mathrm{GeV}$. The region between 1 and $2.5 \mathrm{GeV}$ is at present the most poorly known and is crucial especially for the hadronic corrections to the effective fine structure constant at the $M_{Z}$ scale. In order to improve the theoretical accuracy of $a_{\mu}^{\mathrm{HLO}}$ the precision measurement of the hadronic cross section close to the $\pi \pi$ threshold is also required. In both regions KLOE-2 can give important contributions bringing the accuracy on $a_{\mu}^{\mathrm{HLO}}$ to about $3 \times 10^{-10}$. The result can be achieved by measuring both i) the ratio $\pi^{+} \pi^{-}(\gamma)$ to $\mu^{+} \mu^{-}(\gamma)$ with $0.4 \%$ accuracy at $1 \mathrm{GeV}$ with ISR and ii) the hadronic cross section in the $[1-2.5] \mathrm{GeV}$ region with $[1-2] \%$ fractional error. This would represent a factor of two improvement on the error of $a_{\mu}^{\mathrm{HLO}}$, needed to clarify the $3-\sigma$ discrepancy in conjuction with the proposed muon $g-2$ experiments at FNAL 365] and J-PARC 452. 


\section{Physics in the Continuum: $\gamma \gamma$ Processes}

The upgrade of the KLOE detector with the installation of four stations 453 to tag electrons and positrons from the reaction

$$
e^{+} e^{-} \rightarrow e^{+} e^{-} \gamma^{*} \gamma^{*} \rightarrow e^{+} e^{-} X
$$

gives the opportunity to investigate $\gamma \gamma$ physics at DA $\Phi$ NE . Since it was realised in 1970s that significant production rates could be achieved [454,455, the two photon processes have been investigated at most of the $e^{+} e^{-}$colliders with the limitations imposed by the rate of these subleading processes at the luminosity reached by pastgeneration colliders.

In the $\gamma \gamma$ processes $C=+1$ hadronic states can be produced. In the energy region accessible with the KLOE2 taggers the two $\gamma^{*}$ can be considered quasi-real so that only $J^{P C}=0^{ \pm+}, 2^{ \pm+}$quantum numbers are allowed 456 . If no cut is applied to the final-state leptons, the Weizsäcker Williams or Equivalent Photon Approximation (EPA) 455 can be used to understand the main qualitative features of the process (66). Then the event yield, $N_{e e X}$, can be evaluated according to:

$$
N_{e e X}=L_{e e} \int \frac{\mathrm{dF}}{\mathrm{d} \mathrm{W}_{\gamma \gamma}} \sigma_{\gamma \gamma \rightarrow X}\left(\mathrm{~W}_{\gamma \gamma}\right) \mathrm{dW}_{\gamma \gamma},
$$

where $W_{\gamma \gamma}$ is the invariant mass of the two quasi-real photons, $L_{e e}$ is the integrated luminosity, and $\mathrm{d} F / \mathrm{d} W_{\gamma \gamma}$ is the $\gamma \gamma$ flux function:

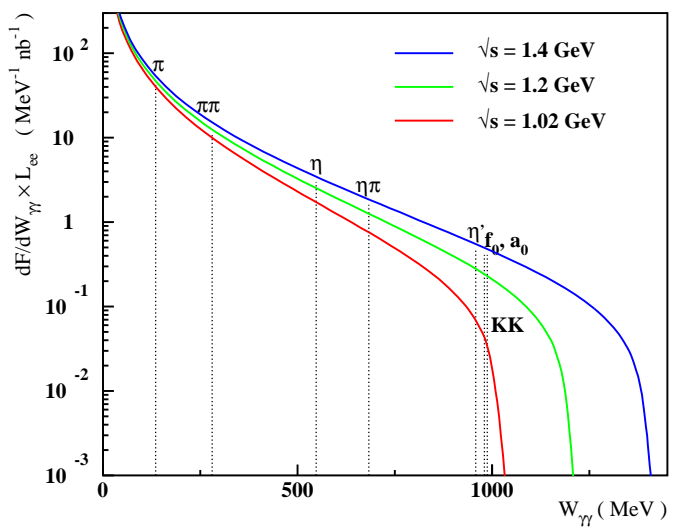

Fig. 25: Differential $\gamma \gamma$ flux function as a function of the center-of-mass energy.

$$
\frac{\mathrm{d} F}{\mathrm{~d} W_{\gamma \gamma}}=\frac{1}{W_{\gamma \gamma}}\left(\frac{2 \alpha}{\pi}\right)^{2}\left(\ln \frac{E_{b}}{m_{e}}\right)^{2} f(z)
$$

where $E_{b}$ is the beam energy and

$$
f(z)=\left(z^{2}+2\right)^{2} \ln \frac{1}{z}-\left(1-z^{2}\right)\left(3+z^{2}\right), \quad z=\frac{\mathrm{W}_{\gamma \gamma}}{2 E_{b}} .
$$

Figure 25] shows the flux function multiplied by an integrated luminosity $L_{e e}=1 \mathrm{fb}^{-1}$, as a function of the $\gamma \gamma$ invariant mass for three different center-of-mass energies. This plot demonstrates the feasibility of the detection of the final states $\pi^{+} \pi^{-}, \pi^{0} \pi^{0}, \pi^{0} \eta$ whose crosssections are of the order of or larger than $1 \mathrm{nb}$ 457, 458, 459,460, 461, and the identification of the resonances produced in these channels. Running DA $\Phi N E$ at centerof-mass energies up to $1.4 \mathrm{GeV}$ can eventually complete the low energy spectroscopy studies with the detection of the heavier $K^{+} K^{-}$and $\bar{K}^{0} K^{0}$ final states. Single pseudoscalar $\left(X=\pi^{0}, \eta\right.$ or $\left.\eta^{\prime}\right)$ production is also accessible and would improve the determination of the two-photon decay widths of these mesons, relevant for the measurement of the pseudoscalar mixing angle $\varphi_{P}$, and the measurement of the valence gluon content in the $\eta^{\prime}$ wavefunction. Moreover, it would be possible to measure the transition form factors $\mathcal{F}_{X \gamma^{*} \gamma^{*}}\left(q_{1}^{2}, q_{2}^{2}\right)$ as a function of the momentum of the virtual photons, $q_{1}^{2}$ and $q_{2}^{2}$. The interest in such form factors is rising again in connection with the theoretical evaluation of the hadronic light-by-light contribution to the muon magnetic anomaly.

\subsection{Scalar resonances in two photon collisions}

The two photon production of hadronic resonances is often advertised as one of the clearest ways of revealing their composition 462, 463, 464, 465, 466, 467, 468, 469, 470, 471, 472, 473, 474, 475, 476, 477]. For instance, the nature of the isoscalar scalars seen in $\pi \pi$ scattering below $1.6 \mathrm{GeV}$, namely the $f_{0}(600)$ or $\sigma, f_{0}(980), f_{0}(1370)$ and $f_{0}(1510)$ mesons, remains an enigma 462,463. While models abound in which some are $\bar{q} q$, some $\overline{q q} q q$, sometimes one is a $\bar{K} K$ molecule, and one a glueball [462, definitive statements are few and far between. Their two photon couplings will help unraveling the enigma.

The ability of photons to probe such structure naturally depends on the photon wavelength. This is readily illustrated by looking at the integrated cross-sections from Mark II 457, Crystal Ball 458 and Belle 460,461 shown in Fig. 26 for $\gamma \gamma \rightarrow \pi^{+} \pi^{-}, \pi^{0} \pi^{0}$. It is worthwhile to consider these processes from the crossed channel viewpoint in which the photon scatters off a pion. At low energies the photon has long wavelength, and so sees the whole hadron and couples to its electric charge. Thus the photon sees the charged pions. The $\pi^{+} \pi^{-}$cross-section is large and its value is a measure of the electric charge of the pion. In contrast, the neutral pion cross-section is small. However, as the energy increases the photon wavelength shortens and recognises that the pions, whether charged or neutral, are made of the same charged constituents, namely quarks, and causes these to resonate, as illustrated in Fig. 27. Thus both channels reveal the well-known $\bar{q} q$ tensor meson, the $f_{2}(1270)$, seen as a peak in Fig. 26, with its production cross-section related to the average charge squared of its constituents.

However, at lower energies, 500-1000 MeV, the photon wavelength is longer. States like the $\sigma$ are so shortlived that they very rapidly disintegrate into two pions 


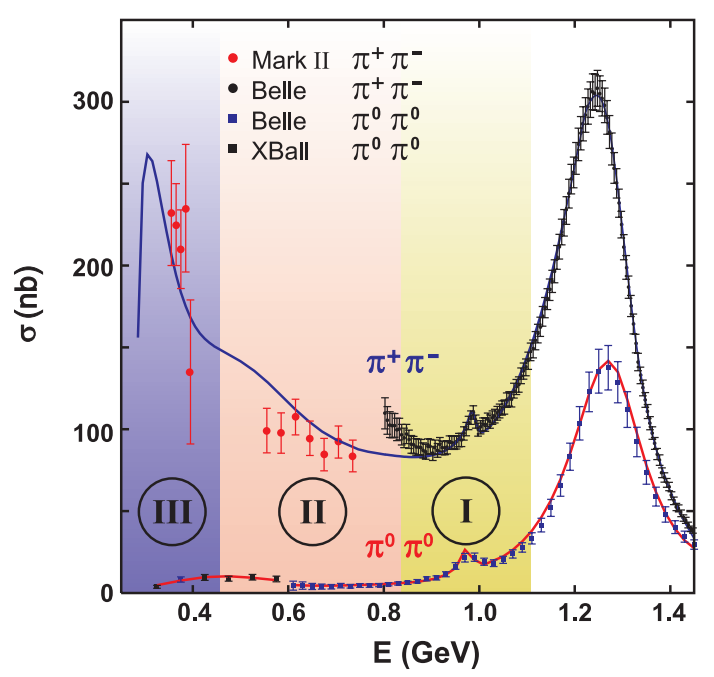

Fig. 26: Cross-section results for $\gamma \gamma \rightarrow \pi^{+} \pi^{-}$from Mark II 457. (below $800 \mathrm{MeV}$ ) and Belle 460 above, integrated over $\left|\cos \theta^{*}\right| \leq 0.6$, and for $\gamma \gamma \rightarrow \pi^{0} \pi^{0}$ from Crystal Ball 458 below $600 \mathrm{MeV}$ and Belle 461 above, integrated over $\left|\cos \theta^{*}\right| \leq 0.8$. $E$ is the $\gamma \gamma$ c.m. energy. The curves are from the (as yet) unpublished Amplitude Analysis that includes the data of Ref. 461. The three shaded bands delineate the energy regions where KLOE-2 can make a contribution discussed in the text.

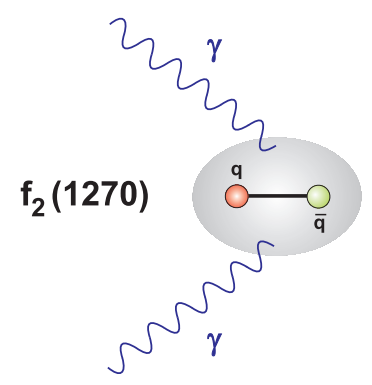

Fig. 27: Illustration of two photons coupling to a relatively long-lived $\bar{q} q$ state. The photons predominantly couples to the electric charge of the constituents.

and when these are charged, the photons couple to these, Fig. 28. The intrinsic make-up of the state, whether $\bar{q} q$, $\overline{q q q q}$ or glueball, is obscured by the large coupling to the pions to which the $\sigma$ decays. Data reveal a similar situation applies to the heavier, and seemingly much narrower, $f_{0}(980)$. This state has equally large hadronic couplings and is only narrow because it sits just below $\bar{K} K$ threshold, to which it strongly couples. Experimental results discussed below suggest the two photons largely see its meson decay products too, regardless of whether the $f_{0}(980)$ is intrinsically a $\bar{K} K$ molecule or not 464, 465, 466, 467, 468, 473, 474, 475, 476, 477.

In the future, strong coupling QCD will eventually predict the two photon couplings of these states according to models of their composition. While theorists work on this, the experimental task is to determine the resonance couplings. To extract these reliably requires a partial wave

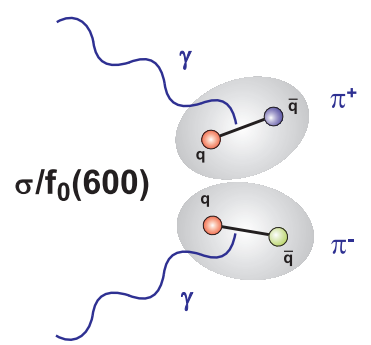

Fig. 28: Illustration of two photons coupling to a state that quickly decays to $\pi \pi$. In the case of the $\sigma / f_{0}(600)$, the photons coupling to the final state dominates over any coupling to the intrinsic make-up of the state, regardless of its composition.

separation. In principle one needs data with full angular coverage with polarised photon beams. But the available data have no polarisation information and in the two photon center-of-mass frame at most $80 \%$ angular acceptance, less in the case of $\pi^{+} \pi^{-}$because of the difficulty of separating these from the scattered $e^{+}$and $e^{-}$. Thus even for the large $f_{2}(1270)$ signal seen so prominently in the integrated cross-sections of Fig. 26, determining its two photon width is not so easy. One must separate the $\pi \pi$ amplitude into components with definite spin, helicity and isospin.

The $\pi^{+} \pi^{-}$cross-section near threshold is dominated by the one pion exchange Born amplitude, producing the enhancement seen in Fig. 26. Being controlled by $I=1$ exchange in the crossed channels means that at low energies $\pi \pi$ production in $I=0$ and $I=2$ must be comparable in all partial waves 478 . Therefore data on both final states, $\pi^{+} \pi^{-}$and $\pi^{0} \pi^{0}$ should be analized at the same time.

The era of high luminosity $e^{+} e^{-}$colliders with their intense programme of study of heavy flavour decays has, as a by-product, yielded two photon data of unprecedented statistics. The Belle collaboration [479,460 has published results on $\gamma \gamma \rightarrow \pi^{+} \pi^{-}$in $5 \mathrm{MeV}$ bins above $800 \mathrm{MeV}$. These show a very clear peak for the $f_{0}(980)$, Fig. 26. Belle 460, analysing just their integrated cross-section, find its radiative width to be $205_{-83-117}^{+95+147} \mathrm{eV}$. The large errors reflect the many ways of drawing a background whether in the $I=0 S$-wave where the resonance appears or in the other partial waves: remember that without full angular coverage the partial waves are not orthogonal, and so interferences occur. Despite these uncertainties, a number of theoretical predictions have now honed in on $[0.2-0.3] \mathrm{keV}$ for the radiative width of the $f_{0}(980)$, whether it is a $\bar{K} K$ molecule or a $\overline{q q} q q$ state $468,469,470$, 475, 476, 477.

The only way to make sense of the real uncertainties is to perform an Amplitude Analysis. A key role is played by the general $S$-matrix properties of analyticity, unitarity and crossing symmetry. When these are combined with the low energy theorem for Compton scattering, these anchor the partial wave amplitudes close to $\pi \pi$ threshold [480. 481,482,483, as described in Refs. 484, 485, 486, 478, and so help to make up for the lack of full angular coverage 
in experiments. Crucially, unitarity imposes a connection between the $\gamma \gamma \rightarrow \pi \pi$ partial wave amplitudes and the behaviour of hadronic processes with $\pi \pi$ final states. Below $1 \mathrm{GeV}$ the unitarity sum is saturated by the $\pi \pi$ intermediate state, while above it the $\bar{K} K$ channel is critically important. Beyond [1.4-1.5] GeV multipion processes start to contribute as $\rho \rho$ threshold is passed. Little is known about the $\pi \pi \rightarrow \rho \rho$ channel in each partial wave. Consequently attention should be restricted to the region below $1.4 \mathrm{GeV}$, where $\pi \pi$ and $\bar{K} K$ intermediate states dominate. The hadronic scattering amplitudes for $\pi \pi \rightarrow \pi \pi$ and $\bar{K} K \rightarrow \pi \pi$ are known and so enable the unitarity constraint to be realised in practice and in turn allow an Amplitude Analysis to be undertaken.

Such an analysis has been performed 478, incorporating all the world data and its key angular information 457, 460, 458, 487, 488. Since the $\pi \pi$ system can be formed in both $I=0$ and $I=2$ final states, the $\pi^{+} \pi^{-}$and $\pi^{0} \pi^{0}$ channels have to be treated simultaneously. Though there are now more than 2000 datapoints in the charged channel below $1.5 \mathrm{GeV}$, there are only 126 in the neutral channel, and they have to be weighted more equally to ensure that the isospin components are reliably separable.

These world data can then be fitted adequately by a range of solutions 478: a range, in which there remains a significant ambiguity in the relative amount of helicity zero $S$ and $D$ waves, particularly above $900 \mathrm{MeV}$. The acceptable solutions have a $\gamma \gamma$ width for the $f_{0}(980)$ (determined from the residue at the pole on the nearby unphysical sheet, being the only unambiguous measure) of between 96 and $540 \mathrm{eV}$, with $450 \mathrm{eV}$ favoured: a significantly larger value than predicted by many current models 466, 467,468, 469, 470, 475, 476, 477]. Of course, the experimental value includes the coupling of the $f_{0}(980)$ to its $\pi \pi$ and $\bar{K} K$ decay products and their final state interactions (the analogue of Fig. 28), not necessarily included in all the theoretical calculations.

The fits accurately follow the lower statistics data from Mark II 457] and CELLO 487] (see the detailed figures in Ref. 478). However, they do not describe the Belle $\pi^{+} \pi^{-}$data between 850 and $950 \mathrm{MeV}$, as seen in Fig. 26. This "mis-fit" is even more apparent in the angular distributions. In the charged pion channel, there is always a large $\mu^{+} \mu^{-}$background. Though the Belle data have unprecedented statistics, the separation of the $\pi^{+} \pi^{-}$signal is highly sensitive to the $\mu$-pair background. This may well be responsible for the apparent distortion below $1 \mathrm{GeV}$ in Fig. 26.

Since that analysis, Belle have more recently published results 461] (both integrated and differential cross-sections) on $\pi^{0} \pi^{0}$ production in $20 \mathrm{MeV}$ bins, Fig. 26. Again these reveal the $f_{0}(980)$ as a small peak, rather than the shoulder seen in earlier much lower statistics data from Crystal Ball [458,488. A new Amplitude Analysis has been started, which significantly changes the solution space, pushing the allowed amplitudes to those with a larger radiative width for the $f_{0}(980)$. However, the final solutions are not yet available.
Though the Belle experiment represents an enormous stride in two photon statistics, there remains room for KLOE-2 to make a significant contribution in each of the three energy regions displayed as bands in Fig. 26 .

I. 850-1100 MeV: accurate measurement of the $\pi^{+} \pi^{-}$ and $\pi^{0} \pi^{0}$ cross-sections (integrated and differential) are crucially still required, with clean $\mu \mu$ background separation. In addition, any information just above $1 \mathrm{GeV}$ on $\bar{K} K$ production would provide an important constraint on the coupled channel Amplitude Analyses described above. Moreover $\pi^{0} \eta$ studies will complement the results from Belle [489].

II. 450-850 MeV: this is the region where the $\sigma$ pole lies. This is a region almost devoid of precision $\gamma \gamma$ data and so allows a range of interpretations [471, 472, 490, 491, 492. Given the importance of the $\sigma$ for our understanding of strong coupling QCD and the nature of the vacuum, it is crucial to measure $\pi \pi$ production in this region in both charge modes [493,494].

III. 280-450 MeV: though this region is controlled by the Born amplitude with corrections computable by the first few orders of ChPT, it is the domain that anchors the partial wave analyses described here. The Mark II experiment [457] is the only one that has made a special measurement of the normalised cross-section for the $\pi^{+} \pi^{-}$channel near threshold. As seen in Fig. 26, their data have very large error-bars.

\subsection{1 $e^{+} e^{-} \rightarrow e^{+} e^{-} \pi \pi$ at KLOE-2}

KLOE-2 can improve the experimental precision in all three regions of Fig. 26] contributing to the solution of the open questions on low-energy hadron physics with the study of $e^{+} e^{-} \rightarrow e^{+} e^{-} \pi \pi$. This process is a clean electromagnetic probe to investigate the nature of the $\sigma$ meson through the analysis of the $\pi \pi$ invariant mass which is expected to be plainly affected by the presence of the scalar meson. A precision measurement of the cross-section of $\gamma \gamma \rightarrow \pi^{+} \pi^{-}$and $\gamma \gamma \rightarrow \pi^{0} \pi^{0}$ would also complete the information from previous experiments allowing the determination of the $\gamma \gamma$ couplings of the $\sigma$ and $f_{0}(980)$ from the partial wave analysis discussed above.

From the experimental point of view the final state with neutral pions, $e^{+} e^{-} \rightarrow e^{+} e^{-} \pi^{0} \pi^{0}$, is cleaner than $e^{+} e^{-} \rightarrow e^{+} e^{-} \pi^{+} \pi^{-}$, the latter being affected by the large background from $e^{+} e^{-} \rightarrow e^{+} e^{-} \mu^{+} \mu^{-}$and from the ISR process $e^{+} e^{-} \rightarrow \pi^{+} \pi^{-} \gamma^{*} \rightarrow \pi^{+} \pi^{-} e^{+} e^{-}$. In Fig. 29, the low-energy part of the $\gamma \gamma \rightarrow \pi^{0} \pi^{0}$ cross-section corresponding to the energy regions II and III of Fig. 26] is shown. The large uncertainties in the data do not allow any conclusion to be drawn about the existence of a resonance-like structure in the region [400-500] MeV.

All the studies to assess the KLOE-2 potentiality in the analysis of $e^{+} e^{-} \rightarrow e^{+} e^{-} \pi^{0} \pi^{0}$ were performed with the MC generator 495, based on the full matrix element calculation over the four-body phase space. The $\sigma \gamma \gamma$ coupling depicted in Fig. 30 is obtained assuming vector meson dominance: the $\sigma$ meson decays to $\rho \rho$ with transitions 


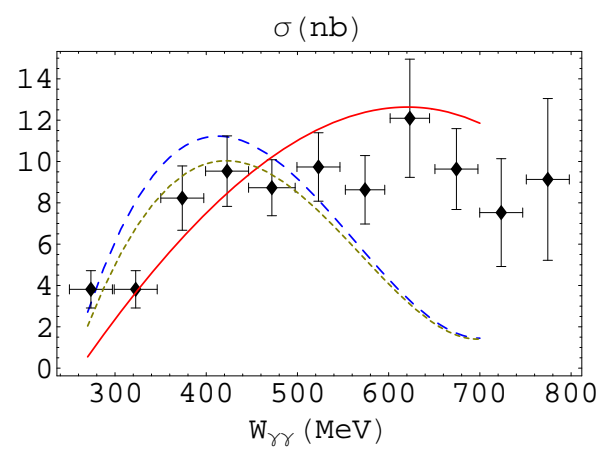

Fig. 29: $\sigma\left(\gamma \gamma \rightarrow \pi^{0} \pi^{0}\right)$ : Crystal Ball data compared with a two-loop ChPT prediction (solid curve), and with the cross-section of $\gamma \gamma \rightarrow \sigma \rightarrow \pi^{0} \pi^{0}$ with (dotted) and without (dashed) Adler zero, obtained according to Ref. 495.

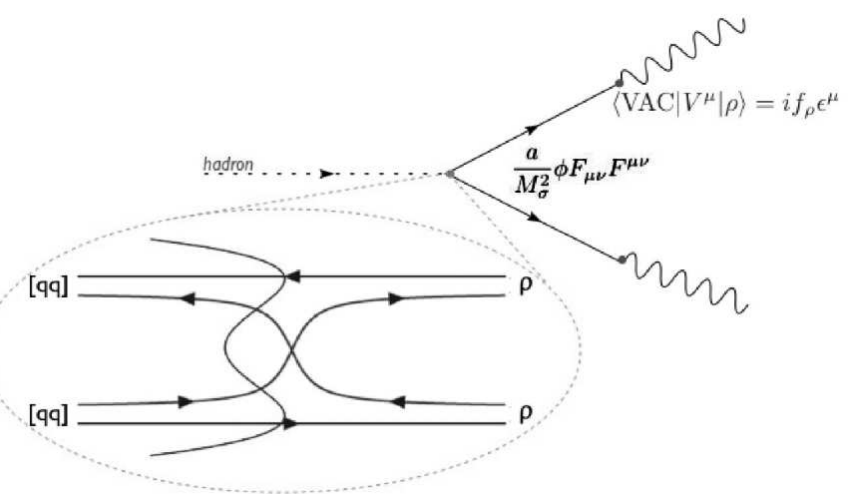

Fig. 30: Schematic view of the $\gamma \gamma \rightarrow \sigma$ transition.

$\rho-\gamma$ whose strength is described by VMD. The underlying dynamics in $\sigma \rightarrow \rho \rho$ is similar to $\sigma \rightarrow \pi \pi$ assuming [286] the $\sigma$ as a bound state of two diquarks: the process is described by the tunneling probability for a quark to escape its diquark shell and bind with an anti-quark of the anti-diquark to form a standard $q \bar{q}$ meson.

Running at $\sqrt{s}=M_{\phi}$ the electron tagger is essential to reduce the large background, mainly from $e^{+} e^{-} \rightarrow \phi \rightarrow$ $K_{S} K_{L}$, with the $K_{L}$ escaping from the detector and the $K_{S}$ decaying to neutral pions, $K_{S} \rightarrow \pi^{0} \pi^{0}$. The tagger is also important to close the kinematics of the process $e^{+} e^{-} \rightarrow e^{+} e^{-} \pi \pi$ allowing a precise reconstruction of the $\gamma \gamma$ invariant mass $\left(\mathrm{W}_{\gamma \gamma}\right)$. Leptons from $\gamma \gamma$ interactions, with $E<510 \mathrm{MeV}$, follow a path through the machine optics different from the orbit of the circulating beams. The trajectories of these off-momentum electrons have been studied by means of a MC simulation, to evaluate their exit point from the DA $\Phi$ NE beam pipe and to find proper location for the tagger devices. This study is based on BDSIM [46], a GEANT4 extension toolkit capable of simulating particle transport in the accelerator beamline. Particles coming from the IP with energies from 5 to 510 $\mathrm{MeV}$ have been simulated with $0.5 \mathrm{MeV}$ step. The results clearly indicate the need to place two different detectors in different regions on both sides of the IP: the Low Energy Tagger (LET) to detect leptons with energy between 150 and $400 \mathrm{MeV}$ and the High Energy Tagger (HET) for those with energy greater than $420 \mathrm{MeV}$. The LET region is one meter from the IP, inside the KLOE magnetic field (Fig. 31). In the LET region the correlation between the energy and the position of the leptons is weak. For this reason the LET detector is a crystal calorimeter able to measure the electron energy with a resolution better than $10 \%$ over the range $[150-400] \mathrm{MeV}$. The HET detector

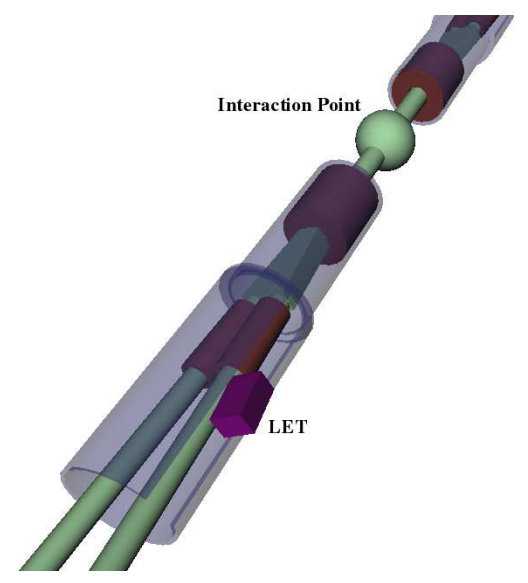

Fig. 31: View of one of the two LET detectors (the most external box) on the DA $\Phi$ NE beam-line.

is located just at the exit of the first bending dipole. In this position the off-momentum electrons escaping from the beam-pipe show a clear correlation between energy and deviation from the nominal orbit (Fig. 32). Therefore the energy of the particles can be obtained from the displacement with respect to the machine orbit measured by a position detector. The preliminary results of the MC

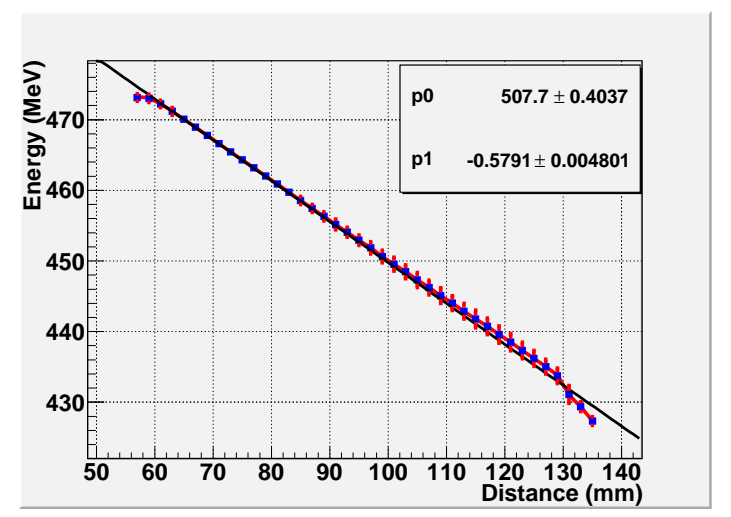

Fig. 32: Energy vs deviation from the nominal orbit for leptons arriving on the HET.

simulation of the tagger stations show that the whole interesting range of the $\gamma \gamma$ invariant mass (zones II and 
III of Fig. 26) is covered by either the LET/LET or the LET/HET coincidences, the latter signaling events from $\pi \pi$ threshold to $800 \mathrm{MeV}$ with maximal acceptance over the $M_{\gamma \gamma}$ range from [600-760] MeV. A LET/LET coincidence indicates instead a $\gamma \gamma$ event at $M_{\gamma \gamma}<350 \mathrm{MeV}$ with acceptance decreasing as invariant mass increases. Using both, data coming from the taggers, and from the KLOE detector, the background can be reduced without relevant signal loss.

For feasibility studies and to optimize the selection algorithms of $\gamma \gamma \rightarrow \pi^{0} \pi^{0}$, about $10 \mathrm{pb}^{-1}$ of KLOE data (i.e., without $e^{ \pm}$taggers) taken at $\sqrt{s}=1 \mathrm{GeV}$ have been used. Four photons compatible with the hypothesis of $\pi^{0} \pi^{0}$ decay with small $(\sim 80 \mathrm{MeV})$ total transverse momentum, were asked for. The distribution of the four-photon invariant mass, $M_{4 \gamma}$, shows an excess of events below 400 $\mathrm{MeV}$ (Fig. 33) not explained by $\phi$ decays or other processes in the continuum.

The excess is due to genuine $e^{+} e^{-} \pi^{0} \pi^{0}$ events and af-

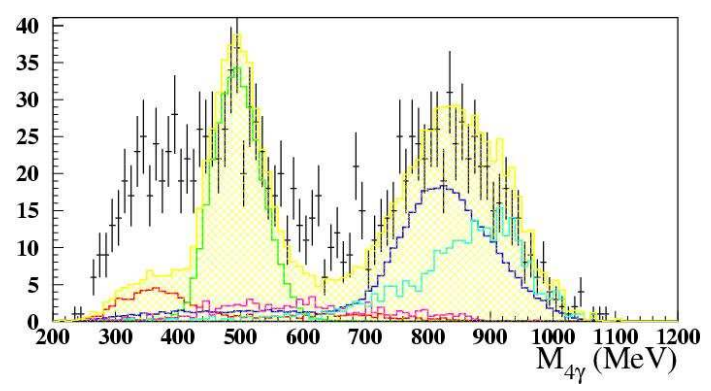

Fig. 33: Four-photon invariant mass: crosses are data, histograms are several sources of background evaluated from MC.

ter proper normalization provides the cross-section. The studies point out that KLOE-2 with an integrated luminosity at the $\phi$ peak of $L=5 \mathrm{fb}^{-1}$ can measure the $\gamma \gamma \rightarrow \pi^{0} \pi^{0}$ cross-section with the same energy binning of Fig. 29 obtained from Crystal Ball [458, reducing the statistical uncertainty in each bin to $2 \%$.

\subsection{Single pseudoscalar final states}

At leading order only two diagrams contribute to the $e^{+} e^{-} \rightarrow$ $e^{+} e^{-} P$ process at the $e^{+} e^{-}$colliders:

$$
\begin{aligned}
& e^{+} e^{-} \rightarrow e^{+} e^{-} \gamma^{*} \gamma^{*} \rightarrow e^{+} e^{-} P \quad(t-\text { channel }) \\
& e^{+} e^{-} \rightarrow \gamma^{*} \rightarrow \gamma^{*} P \rightarrow e^{+} e^{-} P \quad(s-\text { channel })
\end{aligned}
$$

The t-channel with quasi-real photons dominates the production cross-section, however in general the $s$ and the interference contributions are not negligible. The two-photon decay widths of $\pi^{0}, \eta$ and $\eta^{\prime}$ can be extracted from the cross-section of the process (70), of interest among other issues to determine the pseudoscalar mixing angle and improve on the KLOE analysis of the gluonium content in the $\eta^{\prime}$, as discussed in Sect. 4.2.6.
Moreover, the transition form factors $\mathcal{F}_{P \gamma^{*} \gamma^{*}}\left(m_{P}^{2}, q_{1}^{2}, q_{2}^{2}\right)$ at spacelike momentum transfers can be measured from the same process (70). They are important to discriminate among different phenomenological models, relevant for the hadronic light-by-light scattering contribution to the $g-2$ of the muon 383,497.

The $s$-channel contribution of Eq. (71) instead provides information on the timelike form factors, a complementary and promising opportunity to discriminate among alternative models of the underlying dynamics.

\subsubsection{Two photon width of pseudoscalar mesons}

The two-photon decay width $\Gamma(P \rightarrow \gamma \gamma)$ can be measured from the cross-section which in the narrow-width approximation reads 455]:

$$
\sigma_{e^{+} e^{-} \rightarrow e^{+} e^{-P}}=\frac{16 \alpha^{2} \Gamma(P \rightarrow \gamma \gamma)}{m_{P}^{3}}\left(\ln \frac{E_{b}}{m_{e}}\right)^{2} f\left(z_{P}\right),
$$

where $m_{P}$ is the mass of the pseudoscalar meson, $z_{P}=$ $\frac{m_{P}}{2 E_{b}}$ and $f\left(z_{P}\right)$ is given by Eq.(69). Equation (72) is obtained by neglecting the $P \gamma \gamma$ transition form factors, an approximation valid for quasi-real photons. Table 16] shows the cross-section of pseudoscalar meson production at different center-of-mass energies. The $\Gamma(P \rightarrow \gamma \gamma)$ is used as

Table 16: Cross-sections of pseudoscalar meson production at different $\sqrt{s}$ values computed using Eq.(72).

\begin{tabular}{|l||c|c|c||}
\hline$\sqrt{s}(\mathrm{GeV})$ & 1.02 & 1.2 & 1.4 \\
\hline$\sigma_{e^{+} e^{-} \rightarrow e^{+} e^{-} \pi^{0}}(\mathrm{pb})$ & 271 & 317 & 364 \\
\hline$\sigma_{e^{+} e^{-} \rightarrow e^{+} e^{-} \eta}(\mathrm{pb})$ & 45 & 66 & 90 \\
\hline$\sigma_{e^{+} e^{-} \rightarrow e^{+} e^{-} \eta^{\prime}}(\mathrm{pb})$ & 4.9 & 20 & 40 \\
\hline
\end{tabular}

input for:

i. the pseudoscalar mixing angle $\left(\varphi_{P}\right)$ determination from [283,

$$
\begin{aligned}
& \frac{\Gamma(\eta \rightarrow \gamma \gamma)}{\Gamma\left(\pi^{0} \rightarrow \gamma \gamma\right)}=\left(\frac{m_{\eta}}{m_{\pi^{0}}}\right)^{3} \frac{1}{9}\left(5 \cos \varphi_{P}-\sqrt{2} \frac{\bar{m}}{m_{s}} \sin \varphi_{P}\right)^{2} \\
& \frac{\Gamma\left(\eta^{\prime} \rightarrow \gamma \gamma\right)}{\Gamma\left(\pi^{0} \rightarrow \gamma \gamma\right)}=\left(\frac{m_{\eta^{\prime}}}{m_{\pi^{0}}}\right)^{3} \frac{1}{9}\left(5 \sin \varphi_{P}+\sqrt{2} \frac{\bar{m}}{m_{s}} \cos \varphi_{P}\right)^{2}
\end{aligned}
$$

ii. the test of the valence gluon content $\left(Z_{G}^{2}=\sin ^{2} \phi_{\mathrm{G}}\right)$ in the $\eta^{\prime}$ wavefunction 498,283 ,

$$
\begin{gathered}
\frac{\Gamma\left(\eta^{\prime} \rightarrow \gamma \gamma\right)}{\Gamma\left(\pi^{0} \rightarrow \gamma \gamma\right)}=\left(\frac{m_{\eta^{\prime}}}{m_{\pi^{0}}}\right)^{3} \frac{1}{9} \cos ^{2} \phi_{G} \\
\left(5 \sin \varphi_{P}+\sqrt{2} \frac{f_{n}}{f_{s}} \cos \varphi_{P}\right)^{2}
\end{gathered}
$$


$e^{+} e^{-} \rightarrow e^{+} e^{-} \pi^{0}$

For an efficient collection of this kind of events where the $\pi^{0}$ is mostly detected by one of the end-cap calorimeters, KLOE-2 has to overcome some limitations of the KLOE data-taking originated from the triggering criteria and software event filtering. The latter can be improved modifying the rejection procedure for events with unbalanced energy deposit in one of the end-cap calorimeters. The trigger requirement of having at least two energy deposits not in the same end-cap calorimeter produces a distortion in the $\pi^{0}$ energy spectrum and limits the global efficiency for $e^{+} e^{-} \rightarrow e^{+} e^{-} \pi^{0}$ to $20 \%$. Modifications in the KLOE trigger which somewhat soften the requirements on the energy deposit in the calorimeter for events tagged by the LET stations are under evaluation. In addition, the $e^{ \pm}$taggers at KLOE-2 allow the suppression of the background from electroproduction of $\pi^{0}$ 's $\left(\sigma\left(e N \rightarrow e \pi^{0} X\right) \sim \mathcal{O}(1 \mu \mathrm{b})\right)$.

$e^{+} e^{-} \rightarrow e^{+} e^{-} \eta$

Two different final states, $e^{+} e^{-} \gamma \gamma$ and $e^{+} e^{-} \pi^{+} \pi^{-} \pi^{0}$ can be considered. The KLOE analysis of the latter process, based on an integrated luminosity of $240 \mathrm{pb}^{-1}$, is underway using data taken at $\sqrt{s}=1 \mathrm{GeV}$, off the $\phi$ meson peak. Selection criteria consist of two prompt photons and two tracks with opposite curvature. Main background processes are:

$-e^{+} e^{-} \rightarrow \eta\left(\rightarrow \pi^{+} \pi^{-} \pi^{0}\right) \gamma$, with the monochromatic photon lost in the beam pipe;

- double radiative Bhabha events (cross-section of about $100 \mathrm{nb})$;

These amount to about $50 \%$ of fractional background in the final sample, that can be reduced with both, analysis refinements, and using $e^{ \pm}$taggers.

Due to the large background from $e^{+} e^{-} \rightarrow \gamma \gamma(\gamma)$, information from $e^{ \pm}$taggers is more crucial for the analysis of $e^{+} e^{-} \rightarrow e^{+} e^{-} \eta$ with $\eta \rightarrow \gamma \gamma$.

Table 17: Maximal energy of the $e^{+} e^{-}$in the final state for different center-of-mass energies.

\begin{tabular}{|l||c|c|c||}
\hline$\sqrt{s}(\mathrm{GeV})$ & 1.02 & 1.2 & 1.4 \\
\hline $\mathrm{E}_{e}^{\max }\left(\pi^{0}\right)(\mathrm{MeV})$ & 500 & 592 & 693 \\
\hline $\mathrm{E}_{e}^{\max }(\eta)(\mathrm{MeV})$ & 363 & 475 & 593 \\
\hline $\mathrm{E}_{e}^{\max }\left(\eta^{\prime}\right)(\mathrm{MeV})$ & 60 & 218 & 373 \\
\hline
\end{tabular}

$e^{+} e^{-} \rightarrow e^{+} e^{-} \eta^{\prime}$

At $\sqrt{s}=1.02 \mathrm{GeV}$, the $e^{+} e^{-}$in the final state have less than $60 \mathrm{MeV}$ momentum and are out of the acceptance of the tagging devices. Table 17 shows the maximal energy for the $e^{+} e^{-}$in the final state related to different pseudoscalar-meson productions and $\sqrt{s}$ values. Taking into account the $e^{+} e^{-} \rightarrow e^{+} e^{-} \eta^{\prime}$ cross-section, the background due to annihilation processes (scaling with $\sim 1 / \mathrm{s}$ ), and the $e^{+} e^{-}$energy spectrum, the ideal conditions for data analysis would be obtained running at $\sqrt{s}=1.4 \mathrm{GeV}$. Radiative return to the $\phi$ meson is not a limiting factor,

Table 18: Event yield for the dominant $\eta^{\prime}$ decay channels obtained at $\sqrt{s}=1.4 \mathrm{GeV}$ and $L=1 \mathrm{fb}^{-1}$.

\begin{tabular}{|l|c|c|r|}
\hline & Final state & $\eta^{\prime}$ fraction (\%) & events \\
\hline$\pi^{+} \pi^{-} \gamma$ & $\pi^{+} \pi^{-} \gamma$ & $29.4 \pm 0.9$ & 12,000 \\
\hline$\pi^{+} \pi^{-} \eta$ & $\pi^{+} \pi^{-} \gamma \gamma$ & $17.5 \pm 0.5$ & 7,000 \\
\hline$\pi^{0} \pi^{0} \eta$ & $\pi^{0} \pi^{0} \pi^{+} \pi^{-} \pi^{0}$ & $4.7 \pm 0.3$ & 2,000 \\
\hline$\omega \gamma$ & $\pi^{+} \pi^{-} \pi^{0} \gamma$ & $2.7 \pm 0.3$ & 1,200 \\
\hline$\gamma \gamma$ & $\gamma \gamma$ & $2.10 \pm 0.12$ & 800 \\
\hline
\end{tabular}

since the cross-section ranges from $10 \mathrm{pb}$ at $\sqrt{s}=1.2 \mathrm{GeV}$ to $4 \mathrm{pb}$ at $\sqrt{s}=1.4 \mathrm{GeV}$. Table 18 shows the event yields achieved at $\sqrt{s}=1.4 \mathrm{GeV}$ with an integrated luminosity of $1 \mathrm{fb}^{-1}$. Final states with no more than two charged tracks have been considered because they are experimentally clean and thus can be selected with high efficiency. Each decay chain provides $\sigma \propto \Gamma\left(\eta^{\prime} \rightarrow X\right) \times \Gamma\left(\eta^{\prime} \rightarrow \gamma \gamma\right)$, thus we can obtain a precision measurement of $\Gamma\left(\eta^{\prime} \rightarrow \gamma \gamma\right)$ from the analysis of the dominant branching fractions of Tab. 18, imposing $\sum B R_{i}=1$.

\section{Monte Carlo for the KLOE-2 experiment}

There are several Monte Carlo generators for $\gamma \gamma$ physics which can be considered in the forthcoming analyses of KLOE-2 data:

i. a code written by A. Courau 499 used, e.g., in Ref. 500,501 for the $e^{+} e^{-} \rightarrow e^{+} e^{-} \pi^{0} \pi^{0}$, and in Ref. 502. for the $e^{+} e^{-} \rightarrow e^{+} e^{-} \pi^{0}$ process;

ii. a code specifically developed by F. Nguyen et al. 495 for the KLOE analysis of the $e^{+} e^{-} \rightarrow e^{+} e^{-} \pi^{0} \pi^{0}$ (Sect. 6.1.1)

iii. TREPS written by S. Uehara 503 and used by the Belle collaboration 479,460;

iv. TWOGAM developed by D. M. Coffman, used by CLEO 504 for the $e^{+} e^{-} \rightarrow e^{+} e^{-} P$, with $P=\pi^{0}, \eta, \eta^{\prime}$;

v. GGResRC used by the BaBar collaboration [505] and based on the Ref. [455] for single-pseudoscalar production, and on Ref. 506] for the two-pion final state;

vi. EKHARA developed by H. Czyz and collaborators 507 , 508,509 ;

vii. GALUGA and GaGaRes written by G. A. Schuler [510, and F. A. Berends and R. van Gulik 511, respectively,

${ }^{3}$ At $\sqrt{s}=1.2 \mathrm{GeV}$ the yield is half than at $\sqrt{s}=1.4 \mathrm{GeV}$ 
for the study of heavy resonances from $\gamma \gamma$ interaction at the LEPII.

The latter two generators describe high-energy physics and adapting them to KLOE-2 is not straightforward. Most of the others are based on the EPA. EPA is useful to identify the main properties of the two photon processes, however it leads to some discrepancies with respect to the exact formulation as shown in Ref. 455, especially for single-pseudoscalar final states. As KLOE-2 aims at the measurement of the form factors with both photons off-shell, MC simulations beyond EPA are desirable to guarantee the accuracy of the theoretical description.

Basic requirements for a $\mathrm{MC}$ generator for $e^{+} e^{-} \rightarrow$ $e^{+} e^{-} P$ studies not restricted to the region where the photons are quasi-real, are:

i. not to use EPA,

ii. include $s$ - and $t$-channel as well as their interference,

iii. have the flexibility to allow user-defined form factors,

iv. have the flexibility to allow KLOE-2 specific kinematical cuts in order to speed up the generation procedure.

Some work is in progress 512 to develop the EKHARA code on this line. In the present version, EKHARA provides a generic description of the $e^{+} e^{-} \rightarrow e^{+} e^{-} \pi^{+} \pi^{-}$process. As it was developed for the background studies for the KLOE measurement of the pion form factor [513, several modifications are needed before it can be used for two photon physics, but it already contains all relevant contributions to the $e^{+} e^{-} \rightarrow e^{+} e^{-} \pi^{+} \pi^{-}$amplitude.

\subsubsection{Contribution to Light-by-Light scattering}

Figure 34 shows one of the six possible photon momenta configuration of the hadronic light-by-light (HLbL) contribution to the muon anomalous magnetic moment $a_{\mu}=$ $\left(g_{\mu}-2\right) / 2$. This configuration is described by the vertex

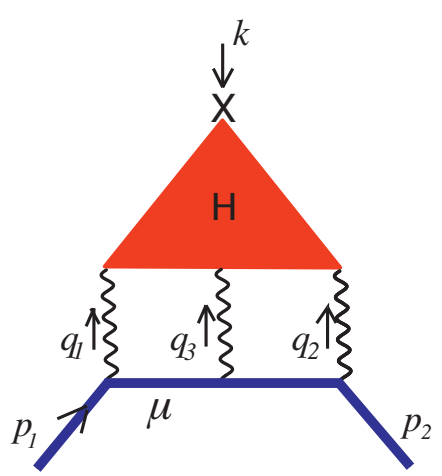

Fig. 34: Hadronic light-by-light scattering contribution.

function

$$
\begin{aligned}
\Gamma^{\mu}\left(p_{2}, p_{1}\right) & =e^{6} \int \frac{\mathrm{d}^{4} q_{1}}{(2 \pi)^{4}} \int \frac{\mathrm{d}^{4} q_{2}}{(2 \pi)^{4}} \frac{\Pi^{\mu \nu \rho \sigma}\left(k, q_{1}, q_{3}, q_{2}\right)}{q_{1}^{2} q_{2}^{2} q_{3}^{2}} \\
& \times \gamma_{\nu}\left(\not p_{2}+\not q_{2}-m_{\mu}\right)^{-1} \gamma_{\rho}\left(\not p_{1}-\not q_{1}-m_{\mu}\right)^{-1} \gamma_{\sigma}
\end{aligned}
$$

where $k$ is the momentum of the photon that couples to the external magnetic source, $k=p_{2}-p_{1}=-q_{1}-q_{2}-q_{3}$ and $m_{\mu}$ is the muon mass. The dominant contribution to the hadronic four-point function

$$
\begin{array}{r}
\Pi^{\rho \nu \alpha \beta}\left(k, q_{1}, q_{2}, q_{3}\right)= \\
i^{3} \int \mathrm{d}^{4} x \int \mathrm{d}^{4} y \int \mathrm{d}^{4} z \mathrm{e}^{i\left(-q_{1} \cdot x+q_{2} \cdot y+q_{3} \cdot z\right)} \\
\times\left\langle 0\left|T\left[V^{\mu}(0) V^{\nu}(x) V^{\rho}(y) V^{\sigma}(z)\right]\right| 0\right\rangle
\end{array}
$$

comes from the three light quark $(q=u, d, s)$ components of the electromagnetic current $V^{\mu}(x)=\left[\bar{q} \hat{Q} \gamma^{\mu} q\right](x)$ where $\hat{Q}$ denotes the quark electric charge matrix. In the limit $k \rightarrow 0$, current conservation implies:

$$
\Gamma^{\mu}\left(p_{2}, p_{1}\right)=-\frac{a^{\mathrm{HLbL}}}{4 m_{\mu}}\left[\gamma^{\mu}, \gamma^{\nu}\right] q_{\nu} .
$$

The main contributions to $a^{\mathrm{HLbL}}$ are summarized in Ref. 402 and reported in the following. Recent reviews of previous work [514,515, 516, 517, 518, 519, 399, 400, 401] are in Refs. [520,521,522,523,524, 405, 383, 525, 404. The discussion in Ref. 402, according to an $1 / N_{c}$ expansion [526], leads to the conclusions :

- Contribution from $\pi^{0}, \eta$ and $\eta^{\prime}$ exchange: implementing a new Operator Product Expansion (OPE) constraint into a neutral pion exchange model, the authors of Ref. 401] obtained $(11.4 \pm 1.0) \times 10^{-10}$. Within the extended Nambu-Jona Lasinio (ENJL) model the momenta higher than a certain cutoff are accounted separately via quark loops [517,518,519, while they are included in the OPE-based model. Assuming that the bulk of high energy quark loops is associated with pseudoscalar exchange, in Ref. [517,518,519] (10.7 \pm $1.3) \times 10^{-10}$ is obtained. Taking into account these results, the authors of Ref. 402 quote as central value the one in [401] with the error as evaluated in Ref. 517, 518, 519:

$$
a^{\mathrm{HLbL}}\left(\pi^{0}, \eta, \eta^{\prime}\right)=(11.4 \pm 1.3) \times 10^{-10} .
$$

- Contribution from pseudovector exchange: the analysis done in Ref.402 suggests that the errors quoted within the large $N_{c}$ ENJL model are underestimated. Taking the average of the estimates and increasing the uncertainty to cover both models, Ref. 402 reports:

$$
a^{\mathrm{HLbL}}(\text { pseudovectors })=(1.5 \pm 1.0) \times 10^{-10} .
$$

- Contribution from scalar exchange: the ENJL model should give a reliable estimate of the large $N_{c}$ contributions, In Ref. 402 therefore the result from 517 , 518 is kept but with a larger conservative error to cover for unaccounted higher-energy resonances providing negative contributions:

$$
a^{\mathrm{HLbL}}(\text { scalars })=-(0.7 \pm 0.7) \times 10^{-10} .
$$


- Contribution from dressed pion and kaon loops: the NLO in $1 / N_{c}$ contributions are the most complicated to calculate. In particular, the charged pion loop shows a large instability due to model dependence. This and the contribution of higher-energy resonance loops are taken into account in Ref. 402 by choosing the central value as the full VMD result of Ref.517,518, with again a conservative error:

$$
a^{\mathrm{HLbL}}\left(\pi^{+}-\text {dressed loop }\right)=-(1.9 \pm 1.9) \times 10^{-10} .
$$

Adding the contributions above as well as the small charm quark contribution and combining errors in quadrature, $a^{\mathrm{HLbL}}($ charm $)=0.23 \times 10^{-10}$, one gets the estimate 402]:

$$
a^{\mathrm{HLbL}}=(10.5 \pm 2.6) \times 10^{-10} .
$$

The proposed new $g_{\mu}-2$ experiment aims at an accuracy of $1.4 \times 10^{-10}$ calling for a considerable improvement on the present calculations. The use of further theoretical and experimental constraints could result in reaching such accuracy soon. In particular, imposing as many as possible short-distance QCD constraints 514,515, 516, 517, 518, 519, 399, 401 has already result in a better understanding of the dominant $\pi^{0}$ exchange. At present, none of the light-by-light hadronic parametrization satisfies fully all short-distance QCD constraints. In particular, this requires the inclusion of infinite number of narrow states for other than two-point functions and two-point functions with soft insertions 527. The dominance of certain momenta configurations can help to minimize the effects of short distance QCD constraints not satisfied, as in the model of Ref. 401.

Recently, the $\pi^{0}$-exchange contribution to $a^{\mathrm{HLbL}}$ has been evaluated from the off-shell form factor $\mathcal{F}_{\pi \gamma^{*} \gamma^{*}}[528$ 403 , obtaining results similar to the ones quoted above. How to take off-shellness effects consistently in the full four-point function of Eq.(74) remains however an open question.

More experimental information on the decays $\pi^{0} \rightarrow$ $\gamma \gamma^{*}, \pi^{0} \rightarrow \gamma^{*} \gamma^{*}$ and $\pi^{0} \rightarrow e^{+} e^{-}$(with radiative corrections included $529,530,531,532$ ) can also help to confirm the results on neutral-pion exchange.

The $\gamma \gamma$ physics program at KLOE- 2 will be well suited to study these processes contributing to solve the present model dependence of $a^{\mathrm{HLbL}}$.

In fact, the dominant contribution from pseudoscalar $(P)$ exchange (Fig. 35) can be written in terms of two form factors, $\mathcal{F}_{P^{*} \gamma^{*} \gamma}$ and $\mathcal{F}_{P^{*} \gamma^{*} \gamma^{*}}$ with one and two offshell photons respectively.

$$
\begin{aligned}
& a^{\mathrm{HLbL}}(P)=-e^{6} \int \frac{d^{4} q_{1}}{(2 \pi)^{4}} \frac{d^{4} q_{2}}{(2 \pi)^{4}} \frac{1}{q_{1}^{2} q_{2}^{2}} \times \\
& \frac{1}{\left(q_{1}+q_{2}\right)^{2}\left[\left(p+q_{1}\right)^{2}-m_{\mu}^{2}\right]\left[\left(p-q_{2}\right)^{2}-m_{\mu}^{2}\right]} \times \\
& {\left[\frac{\mathcal{F}_{P^{*} \gamma^{*} \gamma^{*}}\left(q_{2}^{2}, q_{1}^{2}, q_{3}^{2}\right) \mathcal{F}_{P^{*} \gamma^{*} \gamma}\left(q_{2}^{2}, q_{2}^{2}, 0\right)}{q_{2}^{2}-m_{\pi}^{2}} T_{1}\left(q_{1}, q_{2} ; p\right)+\right.} \\
& \left.\frac{\mathcal{F}_{P^{*} \gamma^{*} \gamma^{*}}\left(q_{3}^{2}, q_{1}^{2}, q_{2}^{2}\right) \mathcal{F}_{P^{*} \gamma^{*} \gamma}\left(q_{3}^{2}, q_{3}^{2}, 0\right)}{q_{3}^{2}-m_{\pi}^{2}} T_{2}\left(q_{1}, q_{2} ; p\right)\right](81)
\end{aligned}
$$
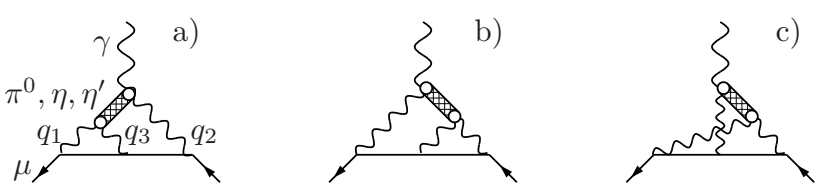

Fig. 35: Pseudoscalar exchange contribution to the light by light scattering.

$T_{1}$ and $T_{2}$ are polynomial function of the photon and pseudoscalar four momenta.

The functional form and the value of the form factors are theoretically unknown. Several models have been used to evaluate them 383, experimental information can be used to constrain both the shape and the value of the form factors.

The form factor at negative $q^{2}$ appears in the production cross section of $\pi^{0}, \eta$ and $\eta^{\prime}$ mesons in the reaction $e^{+} e^{-} \rightarrow e^{+} e^{-} P$. By detecting one electron at large angle with respect to the beams, $\mathcal{F}_{P \gamma \gamma^{*}}\left(m_{P}^{2}, Q^{2}, 0\right)$ with one quasi-real and one virtual spacelike photon $\left(Q^{2}=-q^{2}\right)$ can be measured for the on-shell pseudoscalar. These form factors, as reviewed in Ref. 534, have been measured by the CELLO 535, CLEO 504 and recently BaBar 505 collaborations in the range $\left(1<Q^{2}<40\right) \mathrm{GeV}^{2}$ using single-tagged samples. The experimental data are summarized in Fig. 36. The region below $1 \mathrm{GeV}^{2}$ is still poorly measured and KLOE-2 can cover this region for both $\pi^{0}$ and $\eta$ mesons. Furthermore, by selecting events in which both $e^{+}$and $e^{-}$are detected by the drift chamber KLOE2 can provide experimental information on form factors $\mathcal{F}_{P \gamma^{*} \gamma^{*}}\left(m_{P}^{2}, Q_{1}^{2}, Q_{2}^{2}\right)$, with two virtual photons.

\section{Hidden WIMP Dark Matter}

In recent years, several astrophysical observations have failed to find easy interpretations in terms of standard astrophysical and/or particle physics sources. A non exhaustive list of these observations includes the $511 \mathrm{keV}$ gamma-ray signal from the galactic center observed by the INTEGRAL satellite 536, the excess in the cosmic ray positrons reported by PAMELA [537, the total electron and positron flux measured by ATIC 538, Fermi 539, and HESS [540,541, and the annual modulation of the DAMA/LIBRA signal [542,543.

An intriguing feature of these observations is that they suggest 544,545,546, 547, 548,549,550,551,552,553) the existence of a WIMP dark matter particle belonging to a secluded gauge sector under which the SM particles are uncharged. An abelian gauge field, the $U$ boson with mass near the GeV scale, couples the secluded sector to the SM through its kinetic mixing with the SM hypercharge gauge field The kinetic mixing parameter, $\kappa$, can naturally be of the order $10^{-4}-10^{-2}$.

\footnotetext{
4 The possible existence of a light $U$ boson and the cou-
} pling to the SM particles were already postulated several years 

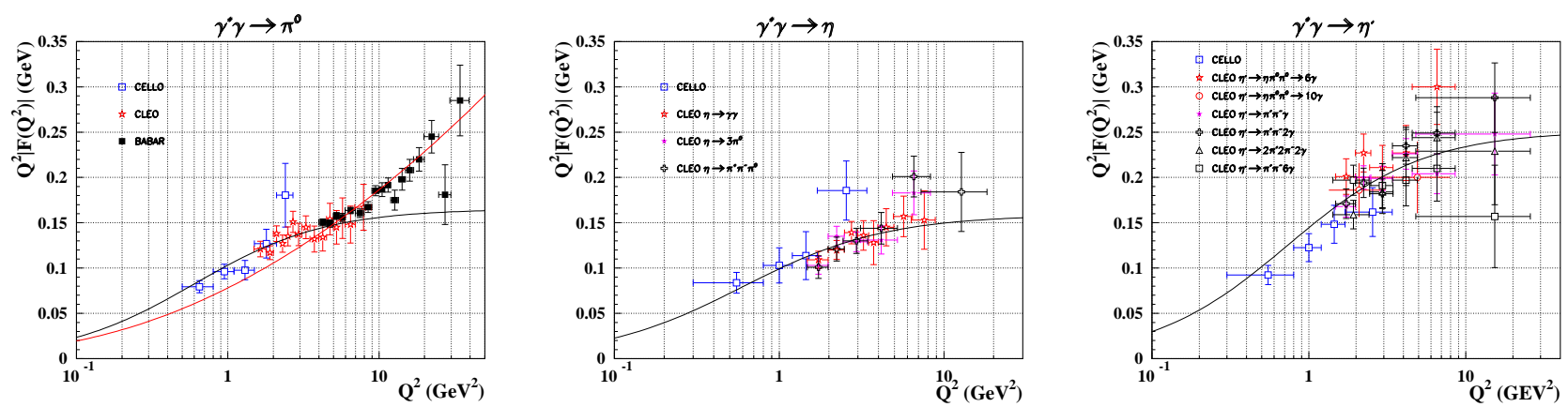

Fig. 36: Left: the $\pi^{0}$ transition form factor as measured by the CELLO, CLEO and BaBar experiments. The curve showing an asymptotic limit at $160 \mathrm{MeV}$ is from CLEO parametrization [504 while the other is from the $\mathcal{F}_{\pi \gamma \gamma^{*}}\left(m_{P}^{2}, Q^{2}, 0\right)$ expression given in Ref. [533. Center: the $\eta$ transition form factor as measured by CELLO and by CLEO in the specified $\eta$ decay channels. Right: the $\eta^{\prime}$ form factor as measured by CELLO and by CLEO in the specified $\eta^{\prime}$ decay channels. The curves in the central and left panel represent the CLEO parametrization for the form factors [504].

Annihilation of DM into the $U$ boson, which decays into charged leptons and is light enough to kinematically forbid a decay that produces anti-protons, can explain the electron and/or positron excesses, and the absence of a similar effect in the PAMELA anti-proton data. The condition $m_{U} \ll m_{\text {WIMP }}$, where mWIMP $_{\text {is }}$ the mass of the DM naturally leads to an enhanced WIMP annihilation cross section in the galaxy, as required by the data. If the DM is also charged under a non-abelian group, higgsed or confined near the GeV scale, then its spectrum naturally implements an inelastic DM scenario [557, thereby explaining the annual modulation signal reported by DAMA/LIBRA 542,543 and reconciling it with the null results of other experiments $545,546,557,558$. In this case, there could be many particles, such as hidden sector higgs and gauge bosons or hidden sector mesons, baryons, and glueballs, with masses near the GeV scale.

A dramatic consequence of the above hypotheses is that observable effects can readily be induced in $\mathcal{O}(\mathrm{GeV})$ energy $\mathrm{e}^{+} \mathrm{e}^{-}$colliders, such as DA $\Phi \mathrm{NE}$, and/or present and future B factories [559,560, 561, 562,563,564 and fixed target experiments [565, 566, 567,568 (c.f. also 569,570, 571,572,573,574, 575,576,577,578). In particular, the $U$ boson can be directly produced at these low-energy facilities, or hidden sector particles at the $\mathrm{GeV}$ scale could be produced through an off-shell $U$ boson as in Fig. 37.

In a very minimal scenario, in addition to the $U$, it is natural to have a secluded Higgs boson, the $h^{\prime}$, which spontaneously breaks the secluded gauge symmetry. Both the $U$ and the $h^{\prime}$ can be produced at DA $\Phi$ NE, and even in this minimal scenario a rich phenomenology is possible. The mass of the $U$ and $h^{\prime}$ are both free parameters, and the possible decay channels can be very different depend-

ago [554,555, 556], in the framework of supersymmetric extensions of the SM. This boson can have both vector and axialvector couplings to quarks and leptons; however, axial couplings are strongly constrained by data, leaving room for vector couplings only.
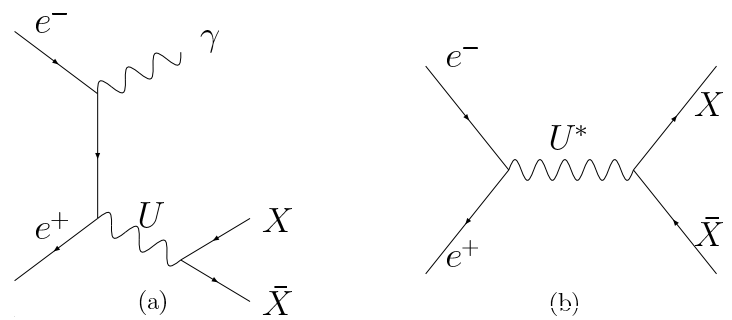

(b)

Fig. 37: Production modes of light secluded-sector particles $X \bar{X}$ at DA $\Phi$ NE. Right: Production through an offshell $U$. Left: Production of an on-shell $U$ and a photon the $U$ may subsequently decay into lighter hidden-sector particles.

ing on which particle is heavier. One thus has to consider different detection strategies for the two cases $m_{h^{\prime}}<m_{U}$ and $m_{h^{\prime}}>m_{U}$.

In order for the $U$ and the $h^{\prime}$ to be produced at DA $\Phi$ $\mathrm{NE}$, their masses need to be less than $\mathrm{m}_{\phi}$. This is somewhat a disadvantage with respect to the $B$-factories. Moreover, the integrated luminosity of the $B$-factories is larger than that of DA $\Phi$ NE. However, the production cross-sections scale as $1 / s$, where $s$ is the squared center-of-mass energy, which partially compensates for the lower luminosity of DA $\Phi N E$. In addition, there are scenarios with a confined secluded gauge group, in which searches at DA $\Phi$ NE are easier than at the $B$-factories. A rather comprehensive discussion of these effects can be found in Refs. 559 , 560]. In particular, the authors of Ref. [560] explicitely discuss the physics potential of DA $\Phi N E$. It turns out that a wide spectrum of physics channels can be studied with KLOE, with rather different detection potentials to the $B$-factories, as further discussed in Ref. [562].

One simple and interesting process to be studied is $\mathrm{e}^{+} \mathrm{e}^{-} \rightarrow U \gamma$. The expected cross section can be as high as $\mathcal{O}(\mathrm{pb})$ at DA $\Phi \mathrm{NE}$ energies, as shown in Fig. 38 (top). The on-shell boson can decay into a lepton pair, giving rise to a $l^{+} l^{-} \gamma$ signal. In the figure, black lines corre- 


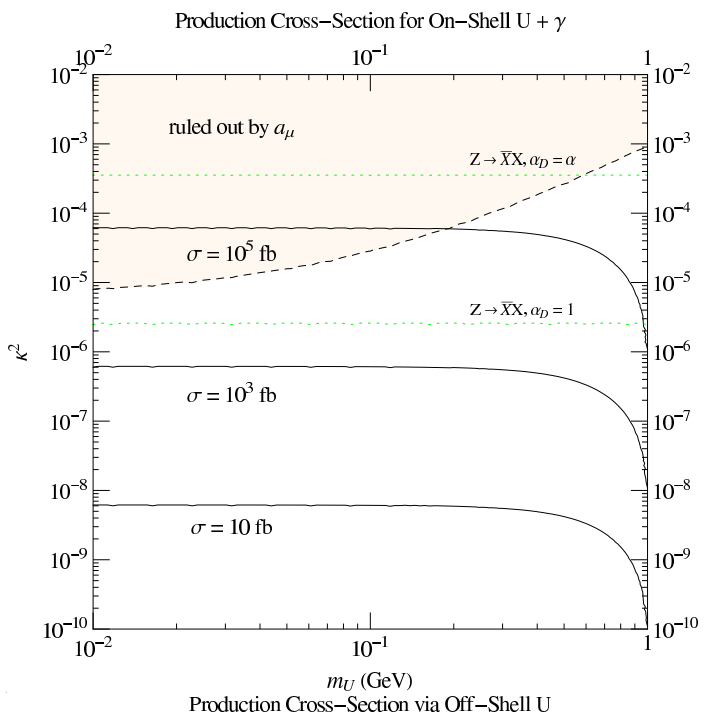

possible instrumental background that has to be taken into consideration for the electron channel is due to $\mathrm{e}^{+} \mathrm{e}^{-}$ $\rightarrow \gamma \gamma$ events with subsequent conversion of one of the two photons on the beam pipe, with a probability of $\sim 2 \%$, that is particularly relevant for low $U$-boson masses. The conclusion of 562 is that a reasonable background rejection can be obtained for $\mathrm{m}_{U} \geq 500 \mathrm{MeV}$.

The insertion of the inner tracker can be rather beneficial for the $\mathrm{e}^{+} \mathrm{e}^{-} \rightarrow U \gamma \rightarrow l^{+} l^{-} \gamma$ case, since it will provide a better definition of the pair production vertex. A quantitative statement on this issue, however, needs the use of a detailed Monte Carlo simulation, which is at present unavailable. For the muon channel, the above mentioned background is not present. However, one has to take into account the physical process $\mathrm{e}^{+} \mathrm{e}^{-} \rightarrow \pi \pi \gamma$, that is relevant since $\pi$ - $\mu$ separation is non-trivial at DA $\Phi$ NE energies. In general, the proposed detector upgrade for the second phase of KLOE-2 should be beneficial increasing acceptance both for charged tracks, thanks to the IT, and for photons, thanks to the forward calorimeters.

In Fig. 38 (bottom), the cross section for the production of an off-shell $U$ with subsequent decay of the $U$ into lighter hidden-sector particles is shown. In this case, the cross section is a function of the product between $\kappa$ and the coupling constant of the dark symmetry $\alpha_{D}$. The signature of these events is more complex than the previous one, depending on the available channels for the hiddensector particles. The case of multilepton channels will be briefly discussed further on.

Assuming the existence of the $h^{\prime}$, a particularly interesting process from the experimental point of view is the $h^{\prime}$-strahlung, $\mathrm{e}^{+} \mathrm{e}^{-} \rightarrow U h^{\prime}$, which can be observed at KLOE if $m_{U}+m_{h^{\prime}}<m_{\phi}$. As stated above, the signature of this process heavily depends on the relation between $m_{U}$ and $m_{h^{\prime}}$. Assuming that the $h^{\prime}$ is lighter than the $U$ boson it turns out to be very long-lived [559] so that the signature of the process will be a lepton pair, generated by the $U$ boson decay, plus missing energy.

There are several advantages for this type of signal. Firstly, there are no other physical processes with the same signature. The background due to QED $l^{+} l^{-} \gamma$ events with a photon lost by the calorimeter, is highly suppressed due to the high detection efficiency of this device. Moreover, this kind of background would give rise to a missing momentum equal to the missing energy, while in the case of the signal these two quantities will be sizeably different, due to the non-zero $h^{\prime}$ mass. In this case, differently from the one discussed above the key ingredient is the very high resolution of the DC as compared to the calorimeter one. A third advantage in terms of both background rejection and detection efficiency is that the leptons tend to be produced at large angle (the angular distribution of the process is proportional to $\sin ^{3} \theta$, for $\mathrm{m}_{h^{\prime}}, \mathrm{m}_{U} \ll \sqrt{s}$ ). Finally, for a wide choice of $\mathrm{m}_{U}$ and $\mathrm{m}_{h^{\prime}}$ the trigger efficiency should exceed $90 \%$.

The only physical process that can give rise to a dangerous background at DA $\Phi$ NE is the process $\phi \rightarrow K_{S}^{0} K_{L}^{0}$ followed by a $K_{S}^{0} \rightarrow \pi^{+} \pi^{-}$decay and the $K_{L}^{0}$ flying through the apparatus without interacting. This decay chain is rele- 
vant only for the $U \rightarrow \mu^{+} \mu^{-}$channel and its contribution can be well calibrated by using the events in which the $K_{L}^{0}$ is observed in the apparatus.

In the case $m_{h^{\prime}}>m_{U}$, the dark higgs frequently decays to a pair of real or virtual $U$ 's. In this case one can observe events with 6 leptons in the final state, due to the $h^{\prime}$-strahlung process, or 4 leptons and a photon, due to the $\mathrm{e}^{+} \mathrm{e}^{-} \rightarrow h^{\prime} \gamma$ reaction. Albeit very spectac-
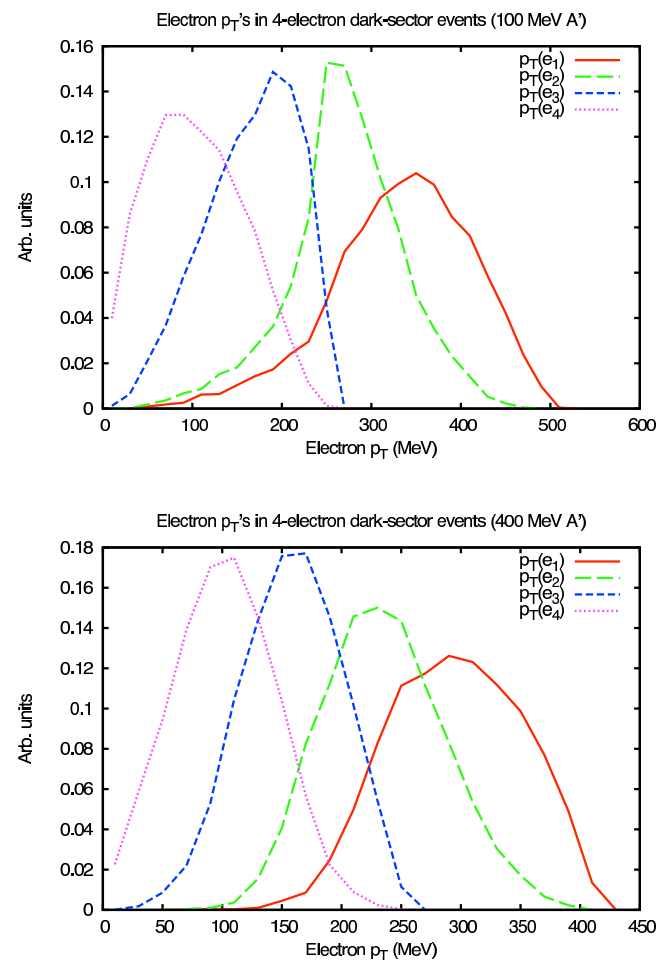

Fig. 39: Electron transverse momentum distributions for the process $e^{+} e^{-} \rightarrow U^{*} \rightarrow W_{D} W_{D} \rightarrow e^{+} e^{-} e^{+} e^{-}$for two $U$-boson masses of $100 \mathrm{MeV}$ (left) and $400 \mathrm{MeV}$ (right). (Here $W_{D}$ refers to two massive hidden sector gauge bosons that mix with the $U$-boson.)

ular, the occurrence of these kind of events at KLOE is limited by the center-of-mass energy, especially for the muon channel. In Fig. 39, we show the electron transverse momentum distributions for the process $e^{+} e^{-} \rightarrow U^{*} \rightarrow$ $W_{D} W_{D} \rightarrow e^{+} e^{-} e^{+} e^{-}$for two $U$-boson masses of $100 \mathrm{MeV}$ (top) and $400 \mathrm{MeV}$ (bottom). For such low multiplicity electron events, it is possible to completely reconstruct the event. However, the higher the multiplicity, the lower is the value of the minimal transverse momentum for the charged particles, resulting in a possible sizable loss of acceptance. In this regard, the insertion of the IT would be clearly beneficial. In order to make more quantitative statements, however, a detailed Monte Carlo simulation is needed for the higher multiplicity lepton signals.

It is important to stress that multilepton events are expected also in more complex models with respect to the one we have so far taken into consideration. For instance
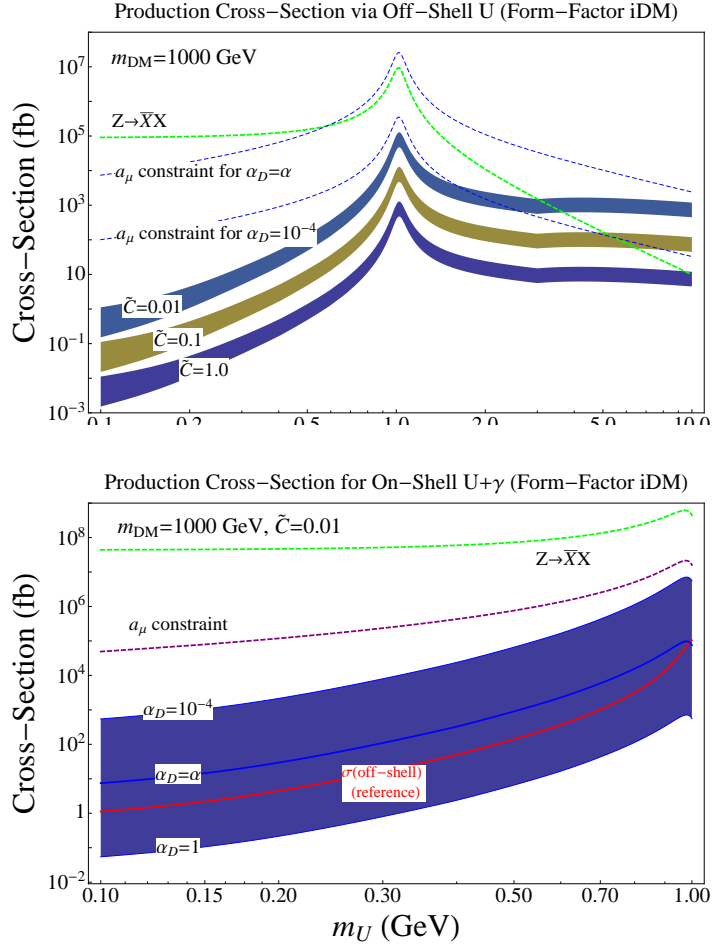

Fig. 40: Inclusive cross-section at DA $\Phi$ NE for production of dark-sector states, $X$, through an off-shell $U$ (top) and for production of an on-shell $U$ with a photon (bottom) as a function of $m_{U}$, after normalizing the couplings to the observed DAMA/LIBRA modulation rate assuming "form-factor-suppressed dipole inelastic dark matter scattering" - see 560] for all the details.

in models with a confined sector, such as the one of Ref. [546, one has hidden sector quarks which can be produced through an off-shell $U$ boson. The cross-section for this process can be very large, of $\mathcal{O}(\mathrm{pb})$ and larger as shown in Fig. 38 (bottom). Once produced, these hidden-sector quarks can confine into hidden-sector vector mesons, that can easily decay back to leptons, and hidden-sector scalar mesons that cannot, so that a possible resulting signal is multileptons plus missing energy. Recently, the BaBar collaboration has searched for 4 lepton events in the three possible channels, $4 e, 4 \mu$ and $2 e 2 \mu$. A preliminary upper limit on $\alpha_{D} \kappa^{2}$ of order $10^{-9}$, slightly dependent on the $\mathrm{W}_{D}$ gauge boson mass, has been set for $M_{W_{D}}$ in the range [0.5-5] GeV [581.

We briefly discuss the implications for DA $\Phi$ NE of taking seriously the inelastic dark matter (iDM) explanation of DAMA/LIBRA and the null results of the other direct detection experiments [557,558. In proposals to generate the iDM splitting among the dark matter states from new non-Abelian gauge interactions in $\mathrm{a} \sim \mathrm{GeV}$-mass hidden sector, it is also natural to assume that scattering is mediated by the $U$-boson 545. In such models, the signal rate and spectrum reported by DAMA/LIBRA constrain the couplings of the $U$ to the SM electromagnetic current. This allows us to estimate the inclusive cross-section for production of hidden-sector states at DA $\Phi$ NE for a given iDM 
model. The expected cross-sections can be $\mathcal{O}(\mathrm{pb})$, at least for $m_{U} \sim \mathrm{GeV}$. However, since the expected production cross-sections scale as $\sim m_{U}^{4}$, very low-mass $U$ 's may evade detection in existing data. The expected cross-sections are shown in Fig. 40, The coefficient $C$ in these figures roughly measures the effective strength of the DM coupling to nuclei, and thus affects the predicted production crosssection at DA $\Phi \mathrm{NE}$. Also shown are the constraints on the couplings of a new $U(1)$ gauge group kinetically mixing with hypercharge from measurements of the muon anomalous magnetic dipole moment 579. The green dotted lines correspond to the lower bounds on the range of couplings that could be probed by a search at LEP for rare $Z$-decays to various exotic final states, assuming that branching ratios as low as $10^{-5}$ can be probed; $m_{D M}$ is set to $1 \mathrm{TeV}$. The upper plot, where $m_{X}=0.2 \mathrm{GeV}$, the number of hidden-sector flavors, $N_{f}=1$, and the number of colors in the hidden sectors, $N_{c}=3$, shows various choices of $\tilde{C}=\{0.01,0.1,1.0\} ;$ in the bottom one, $\alpha_{D}=\left\{1, \alpha, 10^{-4}\right\}$ with $\tilde{C}=0.01$. Note that the off-shell production crosssection scales linearly with the number of dark flavors $N_{f}$ and dark colors $N_{c}$. On both plots constraints from $a_{\mu}$ as well as the rare $Z$ decay sensitivity region are also shown. The details involved in generating this figure are discussed in 560, to which we refer the interested reader.

The $U$ boson can also be produced in radiative decays of neutral mesons, where kinematically allowed. This possibility has been discussed in detail in 561, where several different processes are taken into consideration. It turns out that the $\phi \rightarrow \eta U$ decays can potentially probe couplings down to $\kappa \sim 10^{-3}$, i.e., can cover most of the parameter range interesting for the theory.

The $U$ boson can be observed by its decay into a lepton pair, while the $\eta$ can be tagged by its two-photon or $\pi^{+} \pi^{-} \pi^{0}$ decays. This channel is very attractive from the experimental point of view, since in the final state one has to reconstruct the decays of two particles, which greatly improves background rejection. A possible instrumental background comes from the standard radiative process, $\phi \rightarrow \eta \gamma$, with $\gamma$ conversions on the beam pipe. As for the previously discussed similar cases, the same experimental considerations apply here.

In Figure 41 we present the reach of the U-boson using $\phi \rightarrow \eta U$. Specifically, we estimate the number of background events at given $m_{U}^{2}$ by taking the shape of the background distribution and normalizing it to the PDG branching fraction. Given this number of background events (in a window of size determined by momentum resolution of $0.4 \%$ ), the value of $\epsilon$ for which $S / \sqrt{B}=5$ is plotted. We emphasize that this is only an estimate of possible reach, assuming 8 billion total $\phi$ 's. While it is simple to scale for different integrated luminosity, as the reach is proportional to $\mathcal{L}^{1 / 4}$, the actual reach requires detailed study fully including detector effects. Different choices of form factor significantly modify the reach near the upper end of the kinematically accessible range for $m_{U}$, but have little effect at the low end. The kink in the curve just above $0.2 \mathrm{GeV}$ is from the sudden drop in $U \rightarrow e^{+} e^{-}$branching fraction when the $\mu^{+} \mu^{-}$decay mode opens up. At around

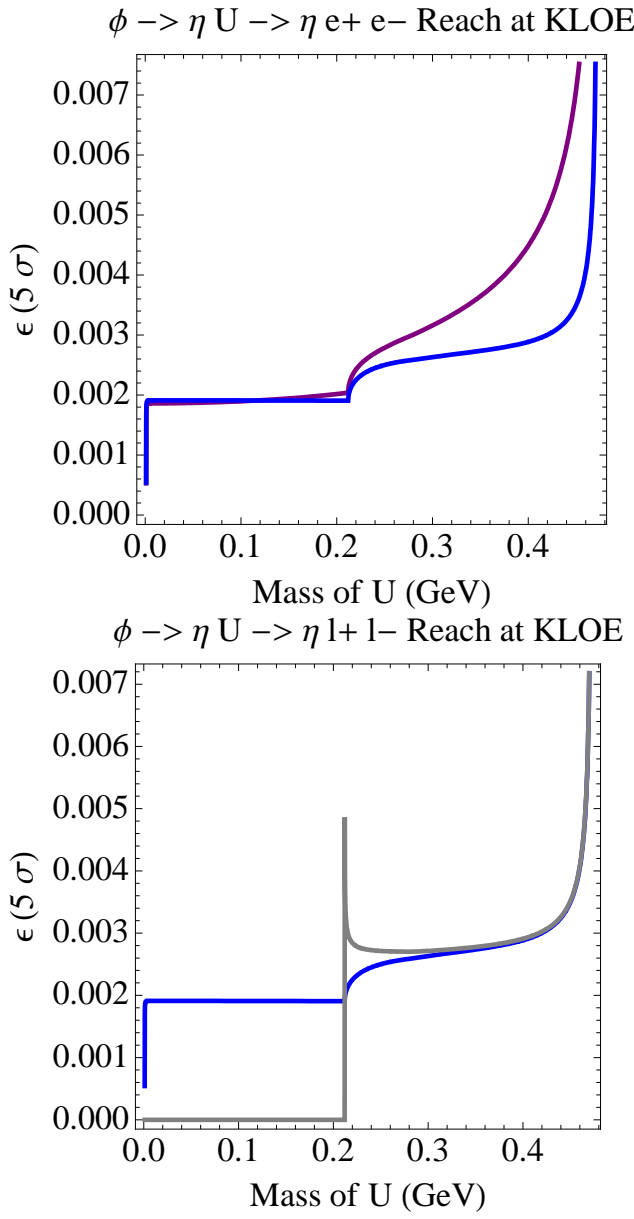

Fig. 41: Reach for $U \rightarrow e^{+} e^{-}$at KLOE in the process $\phi \rightarrow \eta U$. Top: KLOE reach in $e^{+} e^{-}$. The upper curve is for constant form factor $F_{\phi \eta \gamma^{*}}\left(q^{2}\right)=1$, whereas the lower curve is for the single-pole fit $F_{\phi \eta \gamma^{*}}\left(q^{2}\right)=1 /(1-$ $\left.3.8 \mathrm{GeV}^{-2} q^{2}\right)$ from Ref. 268. Bottom: The reach in $e^{+} e^{-}$ with single-pole form factor as above is compared with the corresponding reach in $\mu^{+} \mu^{-}$(gray curve).

$0.28 \mathrm{GeV}$, the decay to $\pi^{+} \pi^{-}$opens up. We estimate its branching ratio using a vector meson dominance model of the pion form factor, $F_{\pi}\left(q^{2}\right)=\frac{1}{1-q^{2} / m_{\rho}^{2}}$, which is approximately valid since we are probing $m_{U} \ll m_{\rho}$. The branching fraction to pions remains small $(\approx 14 \%)$ even at $m_{U} \approx m_{\phi}-m_{\eta}$, so this mode has only a small effect on the reach. After the di-muon threshold, the combined reach of $e^{+} e^{-}$and $\mu^{+} \mu^{-}$is similar to the $e^{+} e^{-}$reach below the threshold.

\section{Summary}

The scientific program at the upgraded $\phi$-factory deserves the attention of particle physicists involved in several different experimental and theoretical projects. 
Lattice QCD and kaon physics are entering a new phase in which experimental measurements and theoretical predictions are sufficiently precise to be sensitive to NP. One of the milestones of KLOE-2 is the improvement of the experimental inputs to $\left|V_{u s}\right|$ and thus the sensitivity of the unitarity test discussed in Sect. 2, for which unquenched lattice calculations are in progress to reduce the uncertainty on the form factors. Interesting enough, precision studies of kinematical distribution of the kaon semileptonic decays can independently provide the $f_{K} / f_{\pi} / f_{+}(0)$ value for the validation of new lattice calculations.

The accomplishment of the entire experimental program, with the completion of the detector upgrades in year 2011 followed by a data-taking campaign to integrate a luminosity of $20 \mathrm{fb}^{-1}$, would allow extensive, unique tests of CPT invariance and QM, investigating on fundamental questions of interest for the quantum theory of gravity.

The program is not limited to kaon physics. Low-energy QCD issues are also addressed with the analysis of $\eta$ and $\eta^{\prime}$ decays and the study of two-photon production of hadronic resonances.

Precision measurements of (multi)hadronic cross sections from $\pi \pi$ threshold to $2.5 \mathrm{GeV}$ are required for the calculation of the hadronic corrections to the fine-structure constant at the $M_{Z}$ scale and for improving the theoretical accuracy of the anomalous magnetic moment of the muon. Progress in this sector is necessary for precision physics at the ILC and to solve the $3-\sigma$ discrepancy between the measurement and the SM prediction of $a_{\mu}$, for which new experiments at the FNAL and J-PARC have been proposed.

The unique opportunity to investigate with proper sensitivity the elusive production of new particles is crucial for probing the hypothesis of a low-mass DM sector.

The list of measurements is not exhaustive but based on several focussing questions triggered by both the experimental and theoretical sides, chosen as benchmarks for planning and operating KLOE-2 at DA $\Phi$ NE.

\section{Acknowledgments}

We thank all the other participants to the workshop on the physics programme at the upgraded DA $\Phi$ NE for their suggestions and useful discussions. The helpful and enjoyable collaboration of the KLOE colleagues is gratefully acknowledged. This work was supported in part by the European Union under contracts of the FP6 Marie Curie Research and Training Networks: FLAVIAnet (MRTN-CT2006-035482), UniverseNet (MRTN-CT-2006-035863), and Hep Tools (MRTN-CT-2006-035505), and by the FP7 Research Infrastructure HadronPhysics2 (INFRA-2008227431). The authors from Jagiellonian University (Cracow) and Soltan Institute for Nuclear Studies (Warsaw) were partially supported by MNiSW Grant No. 0469/B/H03/2009/37. B.C. Hiesmayr gratefully acknowledges the fellowship MOEL 428. J.Prades contribution was partially supported by MICINN, Spain and FEDER, European Commission (EC)- Grant No. FPA2006-05294, by the Spanish Consolider-Ingenio 2010 Programme CPAN - Grant No. CSD2007-00042, and by Junta de Andalucía Grants No. P05-FQM 347 and P07-FQM 03048.

\section{References}

1. C. Milardi et al., ICFA Beam Dyn. Newslett. 48, 23 (2009)

2. M. Zobov et al., ICFA Beam Dyn. Newslett. 48, 34 (2009)

3. R. Beck et al. (KLOE-2) (2006), KLOE-2 Public Documents - K2PD-1, http://www.lnf .infn.it/kloe2/

4. M. Adinolfi et al., Nucl. Instrum. Meth. A488, 51 (2002)

5. M. Adinolfi et al., Nucl. Instrum. Meth. A482, 364 (2002)

6. M. Adinolfi et al. (KLOE), Nucl. Instrum. Meth. A492, $134(2002)$

7. M. Adinolfi et al., Nucl. Instrum. Meth. A483, 649 (2002)

8. A. Aloisio et al., Nucl. Instrum. Meth. A516, 288 (2004)

9. F. Bossi, E. De Lucia, J. Lee-Franzini, S. Miscetti, M. Palutan (KLOE), Riv. Nuovo Cim. 031, 531 (2008), 0811.1929

10. R. Beck et al. (KLOE-2) (2007), Letter of Intent for the KLOE-2 Roll-in, LNF-07/19(IR)

11. F. Archilli et al. (KLOE-2) (2010), 1002.2572

12. W.J. Marciano, A. Sirlin, Phys. Rev. D35, 1672 (1987)

13. K. Hagiwara, S. Matsumoto, Y. Yamada, Phys. Rev. Lett. 75, 3605 (1995), hep-ph/9507419

14. A. Kurylov, M.J. Ramsey-Musolf, Phys. Rev. Lett. 88, 071804 (2002), hep-ph/0109222

15. V. Cirigliano, J. Jenkins, M. Gonzalez-Alonso, Nucl. Phys. B830, 95 (2010), 0908.1754

16. V. Cirigliano, I. Rosell, Phys. Rev. Lett. 99, 231801 (2007), 0707.3439

17. V. Cirigliano, I. Rosell, JHEP 10, 005 (2007), 0707.4464

18. C. Amsler et al. (Particle Data Group), Phys. Lett. B667, $1(2008)$

19. F. Ambrosino et al. (KLOE), Eur. Phys. J. C64, 627 (2009), 0907.3594

20. M. Antonelli et al. (FLAVIAnet working group on kaon decays) (2008), 0801.1817

21. A. Masiero, P. Paradisi, R. Petronzio, Phys. Rev. D74, 011701 (2006), hep-ph/0511289

22. V. Cirigliano, H. Neufeld, H. Pichl, Eur. Phys. J. C35, 53 (2004), hep-ph/0401173

23. V. Cirigliano, M. Knecht, H. Neufeld, H. Rupertsberger, P. Talavera, Eur. Phys. J. C23, 121 (2002), hep-ph/0110153

24. T.C. Andre, Annals Phys. 322, 2518 (2007), hep-ph/0406006

25. V. Cirigliano, M. Giannotti, H. Neufeld, JHEP 11, 006 (2008), 0807.4507

26. M. Ademollo, R. Gatto, Phys. Rev. Lett. 13, 264 (1964)

27. H. Leutwyler, M. Roos, Z. Phys. C25, 91 (1984)

28. J. Bijnens, P. Talavera, Nucl. Phys. B669, 341 (2003), hep-ph/0303103

29. V. Bernard, E. Passemar, Phys. Lett. B661, 95 (2008), 0711.3450

30. D. Becirevic et al., Nucl. Phys. B705, 339 (2005), hep-ph/0403217

31. M. Jamin, J.A. Oller, A. Pich, JHEP 02, 047 (2004), hep-ph/0401080

32. V. Cirigliano et al., JHEP 04, 006 (2005), hep-ph/0503108 
33. A. Kastner, H. Neufeld, Eur. Phys. J. C57, 541 (2008), 0805.2222

34. N. Tsutsui et al. (JLQCD), PoS LAT2005, 357 (2006), hep-lat/0510068

35. C. Dawson, T. Izubuchi, T. Kaneko, S. Sasaki, A. Soni, Phys. Rev. D74, 114502 (2006), hep-ph/0607162

36. D. Brommel et al. (QCDSF), PoS LAT2007, 364 (2007), 0710.2100

37. V. Lubicz, F. Mescia, S. Simula, C. Tarantino (European Twisted Mass), Phys. Rev. D80, 111502 (2009), 0906.4728

38. P.A. Boyle et al., Phys. Rev. Lett. 100, 141601 (2008), 0710.5136

39. P.A. Boyle, J.M. Flynn, A. Juttner, C.T. Sachrajda, J.M. Zanotti, JHEP 05, 016 (2007), hep-lat/0703005

40. C. Aubin et al. (MILC), Phys. Rev. D70, 114501 (2004), hep-lat/0407028

41. A. Bazavov et al. (2009), 0903.3598

42. E. Follana, C. Davies, G.P. Lepage, J. Shigemitsu (HPQCD), Phys. Rev. Lett. 100, 062002 (2008), 0706.1726

43. S. Durr et al. (2010), 1001.4692

44. B. Blossier et al., JHEP 07, 043 (2009), 0904.0954

45. C. Aubin, J. Laiho, R.S. Van de Water (2008), 0810.4328

46. S.R. Beane, P.F. Bedaque, K. Orginos, M.J. Savage, Phys. Rev. D75, 094501 (2007), hep-lat/0606023

47. C. Allton et al. (RBC-UKQCD), Phys. Rev. D78, 114509 (2008), 0804.0473

48. S. Aoki et al. (PACS-CS), Phys. Rev. D79, 034503 (2009), 0807.1661

49. G. Colangelo et al. (FLAVIAnet lattice averaging group - FLAG), PoS KAON09, 029 (2009)

50. F. Ambrosino et al. (KLOE), Phys. Lett. B632, 43 (2006), hep-ex/0508027

51. F. Ambrosino et al. (KLOE), JHEP 02, 098 (2008), 0712.3841

52. F. Ambrosino et al. (KLOE), Phys. Lett. B632, 76 (2006), hep-ex/0509045

53. F. Ambrosino et al. (KLOE), Phys. Lett. B666, 305 (2008), 0804.4577

54. F. Ambrosino et al. (KLOE), Phys. Lett. B636, 173 (2006), hep-ex/0601026

55. F. Ambrosino et al. (KLOE), Eur. Phys. J. C48, 767 (2006), hep-ex/0601025

56. F. Ambrosino et al. (KLOE), Phys. Lett. B626, 15 (2005), hep-ex/0507088

57. F. Ambrosino et al. (KLOE), JHEP 01, 073 (2008), 0712.1112

58. F. Ambrosino et al. (KLOE), Phys. Lett. B636, 166 (2006), hep-ex/0601038

59. F. Ambrosino et al. (KLOE), JHEP 12, 105 (2007), 0710.4470

60. M. Testa et al. (KLOE) (2008), 0805.1969

61. F. Ambrosino et al. (KLOE), JHEP 04, 059 (2008), 0802.3009

62. F. Ambrosino et al. (KLOE), Phys. Lett. B638, 140 (2006), hep-ex/0603041

63. M. Adinolfi et al. (KLOE), Phys. Lett. B566, 61 (2003), hep-ex/0305035

64. F. Ambrosino et al. (KLOE), JHEP 05, 051 (2008), 0712.1744

65. F. Ambrosino et al. (KLOE), Phys. Lett. B619, 61 (2005), hep-ex/0505012
66. F. Ambrosino et al. (KLOE), Phys. Lett. B672, 203 (2009), 0811.1007

67. A. Aloisio et al. (KLOE), Phys. Lett. B597, 139 (2004), hep-ex/0307054

68. S. Bocchetta et al. (KLOE), PoS KAON09, 006 (2009)

69. M. Martini et al. (KLOE-2) (2009), 0906.1133

70. V. Bernard, M. Oertel, E. Passemar, J. Stern, Phys. Lett. B638, 480 (2006), hep-ph/0603202

71. V. Bernard, M. Oertel, E. Passemar, J. Stern, Phys. Rev. D80, 034034 (2009), 0903.1654

72. V. Bernard, M. Oertel, E. Passemar, J. Stern, JHEP 01, 015 (2008), 0707.4194

73. C.G. Callan, S.B. Treiman, Phys. Rev. Lett. 16, 153 (1966)

74. R.F. Dashen, M. Weinstein, Phys. Rev. Lett. 22, 1337 (1969)

75. J. Gasser, H. Leutwyler, Nucl. Phys. B250, 517 (1985)

76. B. Aubert et al. (BaBar), Phys. Rev. D76, 051104 (2007), 0707.2922

77. D. Epifanov et al. (Belle), Phys. Lett. B654, 65 (2007), 0706.2231

78. B. Moussallam, Eur. Phys. J. C53, 401 (2008), 0710.0548

79. M. Jamin, A. Pich, J. Portoles, Phys. Lett. B640, 176 (2006), hep-ph/0605096

80. M. Jamin, A. Pich, J. Portoles, Phys. Lett. B664, 78 (2008), 0803.1786

81. D.R. Boito, R. Escribano, M. Jamin, Eur. Phys. J. C59, 821 (2009), 0807.4883

82. D. Aston et al., Nucl. Phys. B296, 493 (1988)

83. E. Abouzaid et al. (KTeV) (2009), 0912.1291

84. D.R. Boito, R. Escribano, M. Jamin, PoS EFT09, 064 (2009), 0904.0425

85. A. Lai et al. (NA48), Phys. Lett. B647, 341 (2007), hep-ex/0703002

86. S. Descotes-Genon, B. Moussallam, Eur. Phys. J. C42, 403 (2005), hep-ph/0505077

87. M.J. Ramsey-Musolf, S. Su, S. Tulin, Phys. Rev. D76, 095017 (2007), 0705.0028

88. M. Davier, A. Hocker, Z. Zhang, Rev. Mod. Phys. 78, 1043 (2006), hep-ph/0507078

89. W.J. Marciano, Phys. Rev. Lett. 93, 231803 (2004), hep-ph/0402299

90. I.S. Towner, J.C. Hardy, Phys. Rev. C77, 025501 (2008), 0710.3181

91. W.J. Marciano, Phys. Rev. D60, 093006 (1999), hep-ph/9903451

92. W.J. Marciano, PoS KAON, 003 (2008)

93. G. Isidori, P. Paradisi, Phys. Lett. B639, 499 (2006), hep-ph/0605012

94. K. Ikado et al., Phys. Rev. Lett. 97, 251802 (2006), hep-ex/0604018

95. E. Goudzovski (2009), 0908.3858

96. M. Antonelli et al. (2009), 0907.5386

97. G. Luders, Annals Phys. 2, 1 (1957)

98. W. Pauli (1955), in *Enz, C. P. (ed.), Meyenn, K. V. (ed.): Wolfgang Pauli* 459-479

99. J. Bell, Proc. Roy. Soc. Lond. A231, 479 (1955)

100. R. Jost, Helv. Phys. Acta 30, 409 (1957)

101. F. Ambrosino et al. (KLOE), Phys. Lett. B642, 315 (2006), hep-ex/0607027

102. A. Di Domenico (2009), 0904.1976

103. R.A. Bertlmann, K. Durstberger, B.C. Hiesmayr, Phys. Rev. A68, 012111 (2003), quant-ph/0209017 
104. R.A. Bertlmann, W. Grimus, B.C. Hiesmayr, Phys. Rev. A73, 054101 (2006), quant-ph/0602116

105. R. Penrose, Phil. Trans. Roy. Soc. Lond. A356, 1927 (1998)

106. N.E. Mavromatos, PoS KAON, 041 (2008), 0707.3422

107. G.C. Ghirardi, A. Rimini, T. Weber, Phys. Rev. D34, $470(1986)$

108. G.C. Ghirardi, A. Rimini, T. Weber (1987), Trieste ICTP Report IC-87-96

109. G. Lindblad, Commun. Math. Phys. 48, 119 (1976)

110. V. Gorini, A. Kossakowski, E.C.G. Sudarshan, J. Math. Phys. 17, 821 (1976)

111. E. Schrodinger, Naturwiss. 23, 807 (1935)

112. W.H. Furry, Phys. Rev. 49, 393 (1936)

113. R.A. Bertlmann, W. Grimus, Phys. Lett. B392, 426 (1997), hep-ph/9610301

114. R.A. Bertlmann, W. Grimus, B.C. Hiesmayr, Phys. Rev. D60, 114032 (1999), hep-ph/9902427

115. A. Apostolakis et al. (CPLEAR), Phys. Lett. B422, 339 (1998)

116. A. Go et al. (Belle), Phys. Rev. Lett. 99, 131802 (2007), quant-ph/0702267

117. G. Amelino-Camelia (2008), 0806.0339

118. G. Amelino-Camelia, J.R. Ellis, N.E. Mavromatos, D.V. Nanopoulos, S. Sarkar, Nature 393, 763 (1998), astro-ph/9712103

119. R. Gambini, J. Pullin, Phys. Rev. D59, 124021 (1999), gr-qc/9809038

120. R. Aloisio, P. Blasi, P.L. Ghia, A.F. Grillo, Phys. Rev. D62, 053010 (2000), astro-ph/0001258

121. G. Amelino-Camelia, T. Piran, Phys. Rev. D64, 036005 (2001), astro-ph/0008107

122. G. Amelino-Camelia, Int. J. Mod. Phys. D11, 35 (2002), gr-qc/0012051

123. J. Magueijo, L. Smolin, Phys. Rev. D67, 044017 (2003), gr-qc/0207085

124. G. Amelino-Camelia, L. Smolin, A. Starodubtsev, Class. Quant. Grav. 21, 3095 (2004), hep-th/0306134

125. G. Amelino-Camelia, Mod. Phys. Lett. A12, 1387 (1997), gr-qc/9706007

126. G. Amelino-Camelia, S. Majid, Int. J. Mod. Phys. A15, 4301 (2000), hep-th/9907110

127. G. Amelino-Camelia, A. Marciano, M. Arzano (2007), in *Di Domenico, A. (ed.): Handbook of neutral kaon interferometry at a Phi-factory* $155-186$

128. G. Amelino-Camelia, Nature 398, 216 (1999), gr-qc/9808029

129. T. Jacobson, S. Liberati, D. Mattingly, Nature 424, 1019 (2003), astro-ph/0212190

130. U. Jacob, T. Piran, Nature Phys. 3, 87 (2007), hep-ph/0607145

131. G. Amelino-Camelia, F. Mercati (2010), in preparation

132. J. Bernabeu, N.E. Mavromatos, J. Papavassiliou, Phys. Rev. Lett. 92, 131601 (2004), hep-ph/0310180

133. V.A. Kostelecky, ed., CPT and Lorentz symmetry. Proceedings: 4th Meeting, Bloomington, USA, Aug 8-11 (2007)

134. O.W. Greenberg, Phys. Rev. Lett. 89, 231602 (2002), hep-ph/0201258

135. O.W. Greenberg, Found. Phys. 36, 1535 (2006), hep-ph/0309309

136. J.A. Wheeler, K. Ford (1998), Geons, black holes, and quantum foam: A life in physic - New York, USA: Norton (1998) $380 \mathrm{p}$
137. R.M. Wald, Phys. Rev. D21, 2742 (1980)

138. J.R. Ellis, J.S. Hagelin, D.V. Nanopoulos, M. Srednicki, Nucl. Phys. B241, 381 (1984)

139. J.R. Ellis, J.L. Lopez, N.E. Mavromatos, D.V. Nanopoulos, Phys. Rev. D53, 3846 (1996), hep-ph/9505340

140. F. Benatti, R. Floreanini, Nucl. Phys. B488, 335 (1997)

141. P. Huet, M.E. Peskin, Nucl. Phys. B434, 3 (1995), hep-ph/9403257

142. J. Bernabeu, N.E. Mavromatos, J. Papavassiliou, A. Waldron-Lauda, Nucl. Phys. B744, 180 (2006), hep-ph/0506025

143. J. Bernabeu, N.E. Mavromatos, S. Sarkar, Phys. Rev. D74, 045014 (2006), hep-th/0606137

144. N.E. Mavromatos, S. Sarkar, Phys. Rev. D79, 104015 (2009), 0812.3952

145. V.A. Kostelecky, Phys. Rev. D69, 105009 (2004), hep-th/0312310

146. D. Colladay, V.A. Kostelecky, Phys. Rev. D58, 116002 (1998), hep-ph/9809521

147. D. Colladay, V.A. Kostelecky, Phys. Rev. D55, 6760 (1997), hep-ph/9703464

148. V.A. Kostelecky, R. Potting, Phys. Rev. D63, 046007 (2001), hep-th/0008252

149. V.A. Kostelecky, R. Potting, Nucl. Phys. B359, 545 (1991)

150. V.A. Kostelecky, S. Samuel, Phys. Rev. D39, 683 (1989)

151. J. Alfaro, H.A. Morales-Tecotl, L.F. Urrutia, Phys. Rev. Lett. 84, 2318 (2000), gr-qc/9909079

152. F.R. Klinkhamer, C. Rupp, Phys. Rev. D70, 045020 (2004), hep-th/0312032

153. S.M. Carroll, J.A. Harvey, V.A. Kostelecky, C.D. Lane, T. Okamoto, Phys. Rev. Lett. 87, 141601 (2001), hep-th/0105082

154. O. Bertolami, R. Lehnert, R. Potting, A. Ribeiro, Phys. Rev. D69, 083513 (2004), astro-ph/0310344

155. N. Arkani-Hamed, H.C. Cheng, M. Luty, J. Thaler, JHEP 07, 029 (2005), hep-ph/0407034

156. V.A. Kostelecky, R. Lehnert, M.J. Perry, Phys. Rev. D68, 123511 (2003), astro-ph/0212003

157. R. Jackiw, V.A. Kostelecky, Phys. Rev. Lett. 82, 3572 (1999), hep-ph/9901358

158. V.A. Kostelecky, C.D. Lane, A.G.M. Pickering, Phys. Rev. D65, 056006 (2002), hep-th/0111123

159. V.A. Kostelecky, R. Lehnert, Phys. Rev. D63, 065008 (2001), hep-th/0012060

160. R. Lehnert, J. Math. Phys. 45, 3399 (2004), hep-ph/0401084

161. R. Lehnert, Phys. Rev. D68, 085003 (2003), gr-qc/0304013

162. B. Altschul, V.A. Kostelecky, Phys. Lett. B628, 106 (2005), hep-th/0509068

163. R. Bluhm, N.L. Gagne, R. Potting, A. Vrublevskis, Phys. Rev. D77, 125007 (2008), 0802.4071

164. R. Bluhm, V.A. Kostelecky, Phys. Rev. D71, 065008 (2005), hep-th/0412320

165. V.A. Kostelecky, M. Mewes, Phys. Rev. Lett. 87, 251304 (2001), hep-ph/0111026

166. J.Q. Xia, H. Li, X.l. Wang, X.m. Zhang, Astron. Astrophys. 483, 715 (2008), 0710.3325

167. H. Dehmelt, R. Mittleman, R.S. van Dyck, P. Schwinberg, Phys. Rev. Lett. 83, 4694 (1999), hep-ph/9906262

168. M.A. Humphrey et al., Phys. Rev. A68, 063807 (2003), physics/0103068 
169. H. Muller, S. Herrmann, C. Braxmaier, S. Schiller, A. Peters, Phys. Rev. Lett. 91, 020401 (2003), physics $/ 0305117$

170. V.A. Kostelecky, Phys. Rev. Lett. 80, 1818 (1998), hep-ph/9809572

171. H. Nguyen (2001), hep-ex/0112046

172. J.M. Link et al., Phys. Lett. B556, 7 (2003), hep-ex/0208034

173. B. Aubert et al. (BaBar), Phys. Rev. Lett. 100, 131802 (2008), 0711.2713

174. G.W. Bennett et al. (Muon (g-2)), Phys. Rev. Lett. 100, 091602 (2008), 0709.4670

175. M.A. Hohensee, R. Lehnert, D.F. Phillips, R.L. Walsworth, Phys. Rev. Lett. 102, 170402 (2009), 0904.2031

176. J.B.R. Battat, J.F. Chandler, C.W. Stubbs, Phys. Rev. Lett. 99, 241103 (2007), 0710.0702

177. V.A. Kostelecky, N. Russell (2008), 0801.0287

178. V.A. Kostelecky, Phys. Rev. D64, 076001 (2001), hep-ph/0104120

179. V.A. Kostelecky, Phys. Rev. D61, 016002 (2000), hep-ph/9909554

180. V.A. Kostelecky, R. Potting, Phys. Rev. D51, 3923 (1995), hep-ph/9501341

181. B.C. Hiesmayr, M. Huber, Phys. Lett. A372, 3608 (2008), 0711.1368

182. D.M. Greenberger, A. Yasin, Phys. Lett. A138, 391 (1988)

183. B.G. Englert, Phys. Rev. Lett. 77, 2154 (1996)

184. A. Bramon, G. Garbarino, B.C. Hiesmayr, Phys. Rev. A69, 022112 (2004), quant-ph/0311179

185. M.O. Scully, K. Drühl, Phys. Rev. A 25(4), 2208 (1982)

186. Y. Aharonov, M.S. Zubairy, Science 307, 875 (2005)

187. A. Bramon, G. Garbarino, B. Hiesmayr, Phys. Rev. Lett. 92, 020405 (2004), quant-ph/0306114

188. A. Bramon, G. Garbarino, B.C. Hiesmayr, Phys. Rev. A69, 062111 (2004), quant-ph/0402212

189. A. Bramon, G. Garbarino, B.C. Hiesmayr (2004), quant-ph/0404086

190. A. Bramon, G. Garbarino, B.C. Hiesmayr (2003), hep-ph/0311232

191. S. Weinberg, Physica A96, 327 (1979)

192. J. Gasser, H. Leutwyler, Ann. Phys. 158, 142 (1984)

193. G. D'Ambrosio, G. Isidori, Int. J. Mod. Phys. A13, 1 (1998), hep-ph/9611284

194. E. de Rafael (1995), hep-ph/9502254

195. A. Pich, Rept. Prog. Phys. 58, 563 (1995), hep-ph/9502366

196. G. Ecker, Prog. Part. Nucl. Phys. 35, 1 (1995), hep-ph/9501357

197. G. D'Ambrosio, Mod. Phys. Lett. A18, 1273 (2003), hep-ph/0305249

198. G. Ecker, J. Kambor, D. Wyler, Nucl. Phys. B394, 101 (1993)

199. G. D'Ambrosio, J. Portoles, Nucl. Phys. B533, 494 (1998), hep-ph/9711211

200. N. Cabibbo, G. Isidori, JHEP 03, 021 (2005), hep-ph/0502130

201. G. D'Ambrosio, G. Isidori, A. Pugliese, N. Paver, Phys. Rev. D50, 5767 (1994), hep-ph/9403235

202. F. Ambrosino et al. (KLOE), JHEP 12, 011 (2006), hep-ex/0610034

203. G. D'Ambrosio, D. Espriu, Phys. Lett. B175, 237 (1986)
204. F. Buccella, G. D'Ambrosio, M. Miragliuolo, Nuovo Cim. A104, 777 (1991)

205. A. Lai et al. (NA48), Phys. Lett. B551, 7 (2003), hep-ex/0210053

206. G. Buchalla, G. D'Ambrosio, G. Isidori, Nucl. Phys. B672, 387 (2003), hep-ph/0308008

207. A. Lai et al. (NA48), Phys. Lett. B578, 276 (2004), hep-ex/0309022

208. G. Ecker, A. Pich, E. de Rafael, Phys. Lett. B189, 363 (1987)

209. G. Ecker, A. Pich, Nucl. Phys. B366, 189 (1991)

210. A. Angelopoulos et al. (CPLEAR), Phys. Lett. B413, 232 (1997)

211. A.J. Buras, R. Fleischer, S. Recksiegel, F. Schwab, Phys. Rev. Lett. 92, 101804 (2004), hep-ph/0312259

212. G. Ecker, A. Pich, E. de Rafael, Nucl. Phys. B291, 692 (1987)

213. G. D'Ambrosio, G. Ecker, G. Isidori, J. Portoles, JHEP 08, 004 (1998), hep-ph/9808289

214. R. Appel et al. (E865), Phys. Rev. Lett. 83, 4482 (1999), hep-ex/9907045

215. J.R. Batley et al. (NA48/2), Phys. Lett. B677, 246 (2009), 0903.3130

216. J.R. Batley et al. (NA48/1), Phys. Lett. B576, 43 (2003), hep-ex/0309075

217. J.R. Batley et al. (NA48/1), Phys. Lett. B599, 197 (2004), hep-ex/0409011

218. L. Cappiello, G. D'Ambrosio, Phys. Rev. D75, 094014 (2007), hep-ph/0702292

219. G. D'Ambrosio, M. Miragliuolo, P. Santorelli (1992), LNF-92/066 (P)

220. G. D'Ambrosio, G. Isidori, Z. Phys. C65, 649 (1995), hep-ph/9408219

221. M. Raggi et al. (NA48/2), Nucl. Phys.B - Proceedings Supplements 167, 39 (2007), proceedings of BEACH 2006

222. A. Lai et al. (NA48), Eur. Phys. J. C30, 33 (2003)

223. V. Bernard, U.G. Meissner, Ann. Rev. Nucl. Part. Sci. 57, 33 (2007), hep-ph/0611231

224. J. Bijnens, Prog. Part. Nucl. Phys. 58, 521 (2007), hep-ph/0604043

225. J. Flynn, L. Randall, Phys. Lett. B216, 221 (1989)

226. J. Bijnens, Acta Phys. Slov. 56, 305 (2006), hep-ph/0511076

227. E. Witten, Nucl. Phys. B156, 269 (1979)

228. G. 't Hooft, Nucl. Phys. B72, 461 (1974)

229. R. Kaiser, H. Leutwyler, Eur. Phys. J. C17, 623 (2000), hep-ph/0007101

230. A.H. Fariborz, J. Schechter, Phys. Rev. D60, 034002 (1999), hep-ph/9902238

231. A.M. Abdel-Rehim, D. Black, A.H. Fariborz, J. Schechter, Phys. Rev. D67, 054001 (2003), hep-ph/0210431

232. N. Beisert, B. Borasoy, Nucl. Phys. A705, 433 (2002), hep-ph/0201289

233. T. Fujiwara, T. Kugo, H. Terao, S. Uehara, K. Yamawaki, Prog. Theor. Phys. 73, 926 (1985)

234. G. Ecker, J. Gasser, A. Pich, E. de Rafael, Nucl. Phys. B321, 311 (1989)

235. A.V. Anisovich, H. Leutwyler, Phys. Lett. B375, 335 (1996), hep-ph/9601237

236. J. Kambor, C. Wiesendanger, D. Wyler, Nucl. Phys. B465, 215 (1996), hep-ph/9509374 
237. N. Beisert, B. Borasoy, Phys. Rev. D67, 074007 (2003), hep-ph/0302062

238. B. Borasoy, R. Nissler, Eur. Phys. J. A26, 383 (2005), hep-ph/0510384

239. B. Borasoy, R. Nissler, Nucl. Phys. A740, 362 (2004), hep-ph/0405039

240. B. Borasoy, R. Nissler, Eur. Phys. J. A19, 367 (2004), hep-ph/0309011

241. D.G. Sutherland, Phys. Lett. 23, 384 (1966)

242. J.S. Bell, D.G. Sutherland, Nucl. Phys. B4, 315 (1968)

243. H. Leutwyler, Phys. Lett. B378, 313 (1996), hep-ph/9602366

244. W.B. Tippens et al. (Crystal Ball), Phys. Rev. Lett. 87, 192001 (2001)

245. F. Ambrosino et al. (KLOE) (2007), 0707.4137

246. M. Bashkanov et al., Phys. Rev. C76, 048201 (2007), 0708.2014

247. C. Adolph et al. (WASA-at-COSY), Phys. Lett. B677, 24 (2009), 0811.2763

248. M. Unverzagt et al. (Crystal Ball at MAMI), Eur. Phys. J. A39, 169 (2009), 0812.3324

249. S. Prakhov et al. (Crystal Ball at MAMI), Phys. Rev. C79, 035204 (2009), 0812.1999

250. J. Bijnens, K. Ghorbani, JHEP 11, 030 (2007), 0709.0230

251. F. Ambrosino et al. (KLOE), JHEP 05, 006 (2008), 0801.2642

252. D.J. Gross, S.B. Treiman, F. Wilczek, Phys. Rev. D19, 2188 (1979)

253. B. Borasoy, U.G. Meissner, R. Nissler, Phys. Lett. B643, 41 (2006), hep-ph/0609010

254. F.G. Binon et al. (Serpukhov-Brussels-Annecy(LAPP)), Phys. Lett. B140, 264 (1984)

255. D. Alde et al. (Serpukhov-Brussels-Los AlamosAnnecy(LAPP)), Z. Phys. C36, 603 (1987)

256. A.M. Blik et al., Phys. Atom. Nucl. 71, 2124 (2008)

257. P. Naik et al. (CLEO), Phys. Rev. Lett. 102, 061801 (2009), 0809.2587

258. M. Benayoun, P. David, L. DelBuono, P. Leruste, H.B. O'Connell, Eur. Phys. J. C31, 525 (2003), nucl-th/0306078

259. B.R. Holstein, Phys. Scripta T99, 55 (2002) hep-ph/0112150

260. M. Gormley et al., Phys. Rev. D2, 501 (1970)

261. J.G. Layter et al., Phys. Rev. D7, 2565 (1973)

262. B. Borasoy, R. Nissler, Eur. Phys. J. A33, 95 (2007), 0705.0954

263. A. Abele et al. (Crystal Barrel), Phys. Lett. B402, 195 (1997)

264. M. Acciarri et al. (L3), Phys. Lett. B418, 399 (1998)

265. R.I. Dzhelyadin et al., Phys. Lett. B102, 296 (1981)

266. R. Arnaldi et al. (NA60), Phys. Lett. B677, 260 (2009), 0902.2547

267. C. Terschluesen, S. Leupold (2010), 1003.1030

268. M.N. Achasov et al., Phys. Lett. B504, 275 (2001)

269. R. Meijer Drees et al. (SINDRUM-I), Phys. Rev. D45, 1439 (1992)

270. E. Abouzaid et al. (KTeV), Phys. Rev. Lett. 100, 182001 (2008), 0802.2064

271. R.I. Dzhelyadin et al., Phys. Lett. B94, 548 (1980)

272. J. Bijnens, F. Perrsson (1999), hep-ph/0106130

273. F. Ambrosino et al. (KLOE), Phys. Lett. B675, 283 (2009), 0812.4830
274. R.R. Akhmetshin et al. (CMD-2), Phys. Lett. B501, 191 (2001), hep-ex/0012039

275. L.G. Landsberg, Phys. Rept. 128, 301 (1985)

276. D.N. Gao, Mod. Phys. Lett. A17, 1583 (2002), hep-ph/0202002

277. X.G. He, J. Tandean, G. Valencia, JHEP 06, 002 (2008), 0803.4330

278. L. Ametller, J. Bijnens, A. Bramon, F. Cornet, Phys. Lett. B276, 185 (1992)

279. B. Di Micco et al. (KLOE), Acta Phys. Slov. 56, 403 (2006)

280. F. Ambrosino et al. (KLOE), JHEP 07, 105 (2009), 0906.3819

281. J.L. Rosner, Phys. Rev. D27, 1101 (1983)

282. H.Y. Cheng, H.n. Li, K.F. Liu, Phys. Rev. D79, 014024 (2009), 0811.2577

283. F. Ambrosino et al. (KLOE), Phys. Lett. B648, 267 (2007), hep-ex/0612029

284. R. Escribano, J. Nadal, JHEP 05, 006 (2007), hep-ph/0703187

285. R.L. Jaffe, Phys. Rev. D15, 267 (1977)

286. L. Maiani, F. Piccinini, A.D. Polosa, V. Riquer, Phys. Rev. Lett. 93, 212002 (2004), hep-ph/0407017

287. G. 't Hooft, G. Isidori, L. Maiani, A.D. Polosa, V. Riquer, Phys. Lett. B662, 424 (2008), 0801.2288

288. F. Giacosa, Phys. Rev. D74, 014028 (2006), hep-ph/0605191

289. G. Isidori, L. Maiani, M. Nicolaci, S. Pacetti, JHEP 05 049 (2006), hep-ph/0603241

290. D. Black, M. Harada, J. Schechter, Phys. Rev. D73, 054017 (2006), hep-ph/0601052

291. F. Giacosa, G. Pagliara, Nucl. Phys. A812, 125 (2008), 0804.1572

292. N.N. Achasov, A.V. Kiselev, Phys. Rev. D73, 054029 (2006), hep-ph/0512047

293. A. Bramon, R. Escribano, J.L. Lucio M, M. Napsuciale, G. Pancheri, Eur. Phys. J. C26, 253 (2002), hep-ph/0204339

294. R. Escribano, Nucl. Phys. Proc. Suppl. 126, 204 (2004), hep-ph/0307038

295. J.A. Oller, Nucl. Phys. A714, 161 (2003), hep-ph/0205121

296. S. Ivashyn, A. Korchin (2009), 0904.4823

297. A. Heinz, S. Struber, F. Giacosa, D.H. Rischke, Phys. Rev. D79, 037502 (2009), 0805.1134

298. F. Ambrosino et al. (KLOE), Eur. Phys. J. C49, 473 (2007), hep-ex/0609009

299. F. Ambrosino et al. (KLOE), Phys. Lett. B681, 5 (2009), 0904.2539

300. F. Ambrosino et al. (KLOE), Phys. Lett. B634, 148 (2006), hep-ex/0511031

301. A. Aloisio et al. (KLOE), Phys. Lett. B541, 45 (2002), hep-ex/0206010

302. F. Ambrosino et al. (KLOE), Phys. Lett. B679, 10 (2009), 0903.4115

303. D. Hanneke, S. Fogwell, G. Gabrielse, Phys. Rev. Lett. 100, 120801 (2008), 0801.1134

304. F. Jegerlehner, Nucl. Phys. Proc. Suppl. 131, 213 (2004), hep-ph/0312372

305. F. Jegerlehner, Nucl. Phys. Proc. Suppl. 162, 22 (2006), hep-ph/0608329

306. F. Jegerlehner, Nucl. Phys. Proc. Suppl. 181-182, 135 (2008), 0807.4206 
307. W.J. Marciano, A. Sirlin, Phys. Rev. D22, 2695 (1980)

308. A. Sirlin, Phys. Rev. D22, 971 (1980)

309. A. Sirlin, Phys. Lett. B232, 123 (1989)

310. G. Degrassi, S. Fanchiotti, A. Sirlin, Nucl. Phys. B351, 49 (1991)

311. A. Ferroglia, G. Ossola, A. Sirlin, Phys. Lett. B507, 147 (2001), hep-ph/0103001

312. M.J.G. Veltman, Nucl. Phys. B123, 89 (1977)

313. F. Abe et al. (CDF), Phys. Rev. Lett. 74, 2626 (1995), hep-ex/9503002

314. S. Abachi et al. (D0), Phys. Rev. Lett. 74, 2632 (1995), hep-ex/9503003

315. G. Degrassi, P. Gambino, M. Passera, A. Sirlin, Phys. Lett. B418, 209 (1998), hep-ph/9708311

316. G. Degrassi, P. Gambino, Nucl. Phys. B567, 3 (2000), hep-ph/9905472

317. A. Ferroglia, G. Ossola, M. Passera, A. Sirlin, Phys. Rev. D65, 113002 (2002), hep-ph/0203224

318. M. Awramik, M. Czakon, A. Freitas, G. Weiglein, Phys. Rev. D69, 053006 (2004), hep-ph/0311148

319. M. Awramik, M. Czakon, A. Freitas, G. Weiglein, Phys. Rev. Lett. 93, 201805 (2004), hep-ph/0407317

320. M. Awramik, M. Czakon, A. Freitas, JHEP 11, 048 (2006), hep-ph/0608099

321. H. Burkhardt, B. Pietrzyk, Phys. Rev. D72, 057501 (2005), hep-ph/0506323

322. S. Schael et al. (ALEPH), Phys. Rept. 427, 257 (2006), hep-ex/0509008

323. LEP-EW-WG (Last checked 25 February 2010), Electroweak theory tests, http://lepewwg.web.cern.ch

324. G. Weiglein et al. (LHC/LC Study Group), Phys. Rept. 426, 47 (2006), hep-ph/0410364

325. M. Steinhauser, Phys. Lett. B429, 158 (1998), hep-ph/9803313

326. N. Cabibbo, R. Gatto, Phys. Rev. 124, 1577 (1961)

327. S. Eidelman, F. Jegerlehner, Z. Phys. C67, 585 (1995), hep-ph/9502298

328. H. Burkhardt, B. Pietrzyk, Phys. Lett. B356, 398 (1995)

329. A.D. Martin, D. Zeppenfeld, Phys. Lett. B345, 558 (1995), hep-ph/9411377

330. M.L. Swartz, Phys. Rev. D53, 5268 (1996), hep-ph/9509248

331. M. Davier, A. Hoycker, Phys. Lett. B419, 419 (1998), hep-ph/9801361

332. M. Davier, A. Hocker, Phys. Lett. B435, 427 (1998), hep-ph/9805470

333. J.H. Kuhn, M. Steinhauser, Phys. Lett. B437, 425 (1998), hep-ph/9802241

334. S. Groote, J.G. Korner, K. Schilcher, N.F. Nasrallah, Phys. Lett. B440, 375 (1998), hep-ph/9802374

335. J. Erler, Phys. Rev. D59, 054008 (1999), hep-ph/9803453

336. A.D. Martin, J. Outhwaite, M.G. Ryskin, Phys. Lett. B492, 69 (2000), hep-ph/0008078

337. R. Alemany, M. Davier, A. Hocker, Eur. Phys. J. C2, 123 (1998), hep-ph/9703220

338. R.R. Akhmetshin et al. (CMD-2), Phys. Lett. B578, 285 (2004), hep-ex/0308008

339. J.Z. Bai et al. (BES), Phys. Rev. Lett. 88, 101802 (2002), hep-ex/0102003

340. H. Burkhardt, B. Pietrzyk, Phys. Lett. B513, 46 (2001)

341. F. Jegerlehner, J. Phys. G29, 101 (2003), hep-ph/0104304
342. K. Hagiwara, A.D. Martin, D. Nomura, T. Teubner, Phys. Rev. D69, 093003 (2004), hep-ph/0312250

343. J.F. de Troconiz, F.J. Yndurain, Phys. Rev. D71, 073008 (2005), hep-ph/0402285

344. K. Hagiwara, A.D. Martin, D. Nomura, T. Teubner, Phys. Lett. B649, 173 (2007), hep-ph/0611102

345. A. Aloisio et al. (KLOE), Phys. Lett. B606, 12 (2005), hep-ex/0407048

346. R.R. Akhmetshin et al. (CMD-2), JETP Lett. 84, 413 (2006), hep-ex/0610016

347. R.R. Akhmetshin et al. (CMD-2), Phys. Lett. B648, 28 (2007), hep-ex/0610021

348. V.M. Aulchenko et al. (CMD-2), JETP Lett. 82, 743 (2005), hep-ex/0603021

349. M.N. Achasov et al., J. Exp. Theor. Phys. 103, 380 (2006), hep-ex/0605013

350. B. Aubert et al. (BaBar), Phys. Rev. D76, 012008 (2007), 0704.0630

351. B. Aubert et al. (BaBar), Phys. Rev. D76, 092005 (2007), 0708.2461

352. B. Aubert et al. (BaBar), Phys. Rev. D76, 092006 (2007), 0709.1988

353. F. Jegerlehner (1999), hep-ph/9901386

354. S. Eidelman, F. Jegerlehner, A.L. Kataev, O. Veretin, Phys. Lett. B454, 369 (1999), hep-ph/9812521

355. J.H. Kuhn, M. Steinhauser, C. Sturm, Nucl. Phys. B778, 192 (2007), hep-ph/0702103

356. H.N. Brown et al. (Muon (g-2)), Phys. Rev. D62, 091101 (2000), hep-ex/0009029

357. H.N. Brown et al. (Muon g-2), Phys. Rev. Lett. 86, 2227 (2001), hep-ex/0102017

358. G.W. Bennett et al. (Muon g-2), Phys. Rev. Lett. 89, 101804 (2002), hep-ex/0208001

359. G.W. Bennett et al. (Muon g-2), Phys. Rev. Lett. 92, 161802 (2004), hep-ex/0401008

360. G.W. Bennett et al. (Muon g-2), Phys. Rev. D73, 072003 (2006), hep-ex/0602035

361. A. Czarnecki, W.J. Marciano, Phys. Rev. D64, 013014 (2001), hep-ph/0102122

362. D. Stockinger, J. Phys. G34, R45 (2007), hep-ph/0609168

363. D. Stockinger, Nucl. Phys. Proc. Suppl. 181-182, 32 (2008)

364. B.L. Roberts (2010), 1001.2898

365. R.M. Carey et al. (2009), FERMILAB-PROPOSAL-0989

366. T. Kinoshita, M. Nio, Phys. Rev. D73, 013003 (2006), hep-ph/0507249

367. T. Aoyama, M. Hayakawa, T. Kinoshita, M. Nio, Phys. Rev. Lett. 99, 110406 (2007), 0706.3496

368. T. Aoyama, M. Hayakawa, T. Kinoshita, M. Nio, Phys. Rev. D77, 053012 (2008), 0712.2607

369. S. Laporta, E. Remiddi, Phys. Lett. B301, 440 (1993)

370. S. Laporta, E. Remiddi, Phys. Lett. B379, 283 (1996), hep-ph/9602417

371. M. Passera, Phys. Rev. D75, 013002 (2007), hep-ph/0606174

372. A.L. Kataev, Phys. Rev. D74, 073011 (2006), hep-ph/0608120

373. M. Passera, J. Phys. G31, R75 (2005), hep-ph/0411168

374. A. Czarnecki, W.J. Marciano, A. Vainshtein, Phys. Rev. D67, 073006 (2003), hep-ph/0212229

375. A. Czarnecki, B. Krause, W.J. Marciano, Phys. Rev. Lett. 76, 3267 (1996), hep-ph/9512369 
376. A. Czarnecki, B. Krause, W.J. Marciano, Phys. Rev. D52, 2619 (1995), hep-ph/9506256

377. M. Gourdin, E. De Rafael, Nucl. Phys. B10, 667 (1969)

378. T. Teubner, Nucl. Phys. Proc. Suppl. 181-182, 20 (2008)

379. F. Ambrosino et al. (KLOE), Phys. Lett. B670, 285 (2009), 0809.3950

380. M. Davier, Nucl. Phys. Proc. Suppl. 169, 288 (2007), hep-ph/0701163

381. S. Eidelman, Acta Phys. Polon. B38, 3499 (2007)

382. F. Jegerlehner, Nucl. Phys. Proc. Suppl. 181-182, 26 (2008)

383. F. Jegerlehner, A. Nyffeler, Phys. Rept. 477, 1 (2009), 0902.3360

384. B. Aubert et al. (BaBar), Phys. Rev. Lett. 103, 231801 (2009), 0908.3589

385. M. Davier, A. Hoecker, B. Malaescu, C.Z. Yuan, Z. Zhang (2009), 0908.4300

386. M. Davier, S. Eidelman, A. Hocker, Z. Zhang, Eur. Phys. J. C27, 497 (2003), hep-ph/0208177

387. M. Davier, S. Eidelman, A. Hocker, Z. Zhang, Eur. Phys. J. C31, 503 (2003), hep-ph/0308213

388. W.J. Marciano, A. Sirlin, Phys. Rev. Lett. 61, 1815 (1988)

389. A. Sirlin, Nucl. Phys. B196, 83 (1982)

390. V. Cirigliano, G. Ecker, H. Neufeld, Phys. Lett. B513, 361 (2001), hep-ph/0104267

391. V. Cirigliano, G. Ecker, H. Neufeld, JHEP 08, 002 (2002), hep-ph/0207310

392. M. Davier et al., Eur. Phys. J. C66, 127 (2010), 0906.5443

393. F. Flores-Baez, A. Flores-Tlalpa, G. Lopez Castro, G. Toledo Sanchez, Phys. Rev. D74, 071301 (2006), hep-ph/0608084

394. F.V. Flores-Baez, G.L. Castro, G. Toledo Sanchez, Phys. Rev. D76, 096010 (2007), 0708. 3256

395. M. Benayoun, P. David, L. DelBuono, O. Leitner, H.B. O'Connell, Eur. Phys. J. C55, 199 (2008), 0711.4482

396. M. Benayoun, P. David, L. DelBuono, O. Leitner, Eur. Phys. J. C65, 211 (2010), 0907.4047

397. M. Benayoun, P. David, L. DelBuono, O. Leitner (2009), 0907.5603

398. B. Krause, Phys. Lett. B390, 392 (1997) hep-ph/9607259

399. M. Knecht, A. Nyffeler, Phys. Rev. D65, 073034 (2002), hep-ph/0111058

400. M. Knecht, A. Nyffeler, M. Perrottet, E. de Rafael, Phys. Rev. Lett. 88, 071802 (2002), hep-ph/0111059

401. K. Melnikov, A. Vainshtein, Phys. Rev. D70, 113006 (2004), hep-ph/0312226

402. J. Prades, E. de Rafael, A. Vainshtein (2009), 0901.0306

403. A. Nyffeler, Phys. Rev. D79, 073012 (2009), 0901.1172

404. F. Jegerlehner, Acta Phys. Polon. B38, 3021 (2007), hep-ph/0703125

405. J.P. Miller, E. de Rafael, B.L. Roberts, Rept. Prog. Phys. 70, 795 (2007), hep-ph/0703049

406. M. Passera, Nucl. Phys. Proc. Suppl. 155, 365 (2006), hep-ph/0509372

407. M. Passera, Nucl. Phys. Proc. Suppl. 169, 213 (2007), hep-ph/0702027

408. M. Davier, W.J. Marciano, Ann. Rev. Nucl. Part. Sci. 54, $115(2004)$

409. M. Knecht, Lect. Notes Phys. 629, 37 (2004), hep-ph/0307239
410. J. Prades (2009), 0909.2546

411. M. Passera, W.J. Marciano, A. Sirlin, Phys. Rev. D78, 013009 (2008), 0804.1142

412. M. Passera, W.J. Marciano, A. Sirlin (2010), 1001.4528

413. B. Aubert et al. (BaBar), Phys. Rev. D70, 072004 (2004), hep-ex/0408078

414. B. Aubert et al. (BaBar), Phys. Rev. D71, 052001 (2005), hep-ex/0502025

415. A.G. Denig, P.A. Lukin (BaBar), Nucl. Phys. Proc. Suppl. 181-182, 111 (2008)

416. B. Aubert et al. (BaBar), Phys. Rev. D73, 052003 (2006), hep-ex/0602006

417. B. Aubert et al. (BaBar), Phys. Rev. D77, 092002 (2008), 0710.4451

418. A.B. Arbuzov, E.A. Kuraev, N.P. Merenkov, L. Trentadue, JHEP 12, 009 (1998), hep-ph/9804430

419. S. Binner, J.H. Kuhn, K. Melnikov, Phys. Lett. B459, 279 (1999), hep-ph/9902399

420. M. Benayoun, S.I. Eidelman, V.N. Ivanchenko, Z.K. Silagadze, Mod. Phys. Lett. A14, 2605 (1999), hep-ph/9910523

421. G. Rodrigo, A. Gehrmann-De Ridder, M. Guilleaume, J.H. Kuhn, Eur. Phys. J. C22, 81 (2001), hep-ph/0106132

422. J.H. Kuhn, G. Rodrigo, Eur. Phys. J. C25, 215 (2002), hep-ph/0204283

423. G. Rodrigo, H. Czyz, J.H. Kuhn, M. Szopa, Eur. Phys. J. C24, 71 (2002), hep-ph/0112184

424. H. Czyz, A. Grzelinska, J.H. Kuhn, G. Rodrigo, Eur. Phys. J. C27, 563 (2003), hep-ph/0212225

425. H. Czyz, A. Grzelinska, J.H. Kuhn, G. Rodrigo, Eur. Phys. J. C33, 333 (2004), hep-ph/0308312

426. W. Kluge, Nucl. Phys. Proc. Suppl. 181-182, 280 (2008), 0805.4708

427. S. Actis et al. (2009), 0912.0749

428. S. Godfrey, N. Isgur, Phys. Rev. D32, 189 (1985)

429. M.N. Achasov et al., Phys. Rev. D68, 052006 (2003), hep-ex/0305049

430. R.R. Akhmetshin et al. (CMD-2), Phys. Lett. B489, 125 (2000), hep-ex/0009013

431. A. Antonelli et al. (DM2), Z. Phys. C56, 15 (1992)

432. D. Bisello et al. (DM2), Nucl. Phys. Proc. Suppl. 21, 111 (1991)

433. R.R. Akhmetshin et al. (CMD-2), Phys. Lett. B595, 101 (2004), hep-ex/0404019

434. M.N. Achasov et al., J. Exp. Theor. Phys. 101, 1053 (2005), hep-ex/0506076

435. M. Fujikawa et al. (Belle), Phys. Rev. D78, 072006 (2008), 0805.3773

436. T. Barnes, F.E. Close, P.R. Page, E.S. Swanson, Phys. Rev. D55, 4157 (1997), hep-ph/9609339

437. C. Balazs, H.J. He, C.P. Yuan, Phys. Rev. D60, 114001 (1999), hep-ph/9812263

438. S.I. Bityukov et al., Phys. Lett. 188B, 383 (1987)

439. P.L. Frabetti et al., Phys. Lett. B578, 290 (2004), hep-ex/0310041

440. A. Antonelli et al. (FENICE), Phys. Lett. B365, 427 (1996)

441. A. Castro (DM2), Nuovo Cim. A107, 1807 (1994)

442. M. Ablikim et al. (BES), Phys. Rev. Lett. 97, 142002 (2006), hep-ex/0606047

443. F. Mane et al., Phys. Lett. B112, 178 (1982) 
444. C.P. Shen et al. (Belle), Phys. Rev. D80, 031101 (2009), 0808.0006

445. J.M. Link et al. (FOCUS), Phys. Lett. B545, 50 (2002), hep-ex/0208027

446. M. Ablikim et al. (BES), Phys. Rev. Lett. 100, 102003 (2008), 0712.1143

447. S.R. Amendolia et al. (NA7), Nucl. Phys. B277, 168 (1986)

448. S.E. Muller et al. (KLOE) (2009), 0912.2205

449. V.E. Blinov et al., Nucl. Instrum. Meth. A598, 23 (2009)

450. O. Bartalini et al. (GRAAL), Eur. Phys. J. A26, 399 (2005)

451. V.G. Gurzadyan et al., Mod. Phys. Lett. A20, 19 (2005), astro-ph/0410742

452. M. Aoki et al. (2009), J-PARC proposal, http://j-parc.jp/jhf-np/Proposal_e.html

453. D. Babusci et al. (KLOE-2) (2009), 0906.0875

454. S.J. Brodsky, T. Kinoshita, H. Terazawa, Phys. Rev. Lett. 25, $972(1970)$

455. S.J. Brodsky, T. Kinoshita, H. Terazawa, Phys. Rev. D4, $1532(1971)$

456. C.N. Yang, Phys. Rev. 77, 242 (1950)

457. J. Boyer et al., Phys. Rev. D42, 1350 (1990)

458. H. Marsiske et al. (Crystal Ball), Phys. Rev. D41, 3324 (1990)

459. T. Oest et al. (JADE), Z. Phys. C47, 343 (1990)

460. T. Mori et al. (Belle), Phys. Rev. D75, 051101 (2007), hep-ex/0610038

461. S. Uehara et al. (Belle), Phys. Rev. D78, 052004 (2008), 0810.0655

462. E. Klempt, A. Zaitsev, Phys. Rept. 454, 1 (2007), 0708.4016

463. M.R. Pennington (2007), 0711.1435

464. T. Barnes, Phys. Lett. B165, 434 (1985)

465. T. Barnes (1992), invited paper to Int. Workshop on Photon-Photon Collisions, La Jolla, CA, Mar 22-26, 1992

466. N.N. Achasov, G.N. Shestakov, Z. Phys. C41, 309 (1988)

467. N.N. Achasov, A.V. Kiselev, Phys. Rev. D76, 077501 (2007), hep-ph/0606268

468. C. Hanhart, Y.S. Kalashnikova, A.E. Kudryavtsev, A.V. Nefediev, Phys. Rev. D75, 074015 (2007), hep-ph/0701214

469. T. Branz, T. Gutsche, V.E. Lyubovitskij, Phys. Rev. D78, 114004 (2008), 0808.0705

470. M.K. Volkov, E.A. Kuraev, Y.M. Bystritskiy (2009), 0904.2484

471. G. Mennessier, S. Narison, W. Ochs, Phys. Lett. B665, 205 (2008), 0804.4452

472. G. Mennessier, S. Narison, W. Ochs, Nucl. Phys. Proc. Suppl. 181-182, 238 (2008), 0806.4092

473. N.N. Achasov, G.N. Shestakov, Phys. Rev. D77, 074020 (2008), 0712.0885

474. N.N. Achasov, G.N. Shestakov (2009), 0905. 2017

475. F. Giacosa, AIP Conf. Proc. 1030, 153 (2008), 0804.3216

476. F. Giacosa, T. Gutsche, V.E. Lyubovitskij, Phys. Rev. D77, 034007 (2008), 0710.3403

477. E. van Beveren, F. Kleefeld, G. Rupp, M.D. Scadron, Phys. Rev. D79, 098501 (2009), 0811.2589

478. M.R. Pennington, T. Mori, S. Uehara, Y. Watanabe, Eur. Phys. J. C56, 1 (2008), 0803.3389

479. T. Mori et al. (Belle), J. Phys. Soc. Jap. 76, 074102 (2007), 0704.3538
480. D. Morgan, M.R. Pennington, Phys. Lett. B272, 134 (1991)

481. D.H. Lyth, Nucl. Phys. B30, 195 (1971)

482. G. Mennessier, Z. Phys. C16, 241 (1983)

483. G. Mennessier, T.N. Truong, Phys. Lett. B177, 195 (1986)

484. M.R. Pennington (1992), Durham Report DTP-92-32

485. M.R. Pennington (1995), Durham Report DTP-95-18

486. M. Boglione, M.R. Pennington, Eur. Phys. J. C9, 11 (1999), hep-ph/9812258

487. H.J. Behrend et al. (CELLO), Z. Phys. C56, 381 (1992)

488. J.K. Bienlein (Crystal Ball) (1992), Crystal Ball contributions to 9th Int. Workshop on Photon- Photon Collisions, La Jolla, CA, Mar 22-26, 1992

489. S. Uehara et al. (Belle), Phys. Rev. D80, 032001 (2009), 0906.1464

490. M.R. Pennington, Phys. Rev. Lett. 97, 011601 (2006)

491. J.A. Oller, L. Roca, C. Schat, Phys. Lett. B659, 201 (2008), 0708.1659

492. J. Bernabeu, J. Prades, Phys. Rev. Lett. 100, 241804 (2008), 0802.1830

493. M.R. Pennington, Prog. Theor. Phys. Suppl. 168, 143 (2007), hep-ph/0703256

494. M.R. Pennington, Mod. Phys. Lett. A22, 1439 (2007), 0705.3314

495. F. Nguyen, F. Piccinini, A.D. Polosa, Eur. Phys. J. C47, 65 (2006), hep-ph/0602205

496. S. Malton et al. (2008), BdSim MonteCarlo, https://www.pp.rhul.ac.uk/twiki/bin/view/JAI/BdSim

497. J. Prades (2009), 0905.3164

498. E. Kou, Phys. Rev. D63, 054027 (2001), hep-ph/9908214

499. A. Courau (1984), SLAC-PUB-3363

500. G. Alexander et al., Nuovo Cim. A107, 837 (1994)

501. S. Bellucci, A. Courau, S. Ong (1994), LNF-94/078(P)

502. S. Ong (1999), hep-ex/9902030

503. S. Uehara (1996), KEK-REPORT-96-11

504. J. Gronberg et al. (CLEO), Phys. Rev. D57, 33 (1998), hep-ex/9707031

505. B. Aubert et al. (BaBar), Phys. Rev. D80, 052002 (2009), 0905.4778

506. V.M. Budnev, I.F. Ginzburg, G.V. Meledin, V.G. Serbo, Phys. Rept. 15, 181 (1975)

507. H. Czyz, E. Nowak, Acta Phys. Polon. B34, 5231 (2003), hep-ph/0310335

508. H. Czyz, E. Nowak-Kubat, Acta Phys. Polon. B36, 3425 (2005), hep-ph/0510287

509. H. Czyz, E. Nowak-Kubat, Phys. Lett. B634, 493 (2006), hep-ph/0601169

510. G.A. Schuler, Comput. Phys. Commun. 108, 279 (1998), hep-ph/9710506

511. F.A. Berends, R. van Gulik, Comput. Phys. Commun. 144, 82 (2002), hep-ph/0109195

512. H. Czyz, S. Ivashyn (2010), in preparation

513. F. Ambrosino et al. (KLOE), Phys. Lett. B670, 285 (2009), 0809. 3950

514. M. Hayakawa, T. Kinoshita, A.I. Sanda, Phys. Rev. Lett. 75, 790 (1995), hep-ph/9503463

515. M. Hayakawa, T. Kinoshita, A.I. Sanda, Phys. Rev. D54, 3137 (1996), hep-ph/9601310

516. M. Hayakawa, T. Kinoshita, Phys. Rev. D57, 465 (1998), hep-ph/9708227

517. J. Bijnens, E. Pallante, J. Prades, Nucl. Phys. B474, 379 (1996), hep-ph/9511388 
518. J. Bijnens, E. Pallante, J. Prades, Phys. Rev. Lett. 75, 1447 (1995), hep-ph/9505251

519. J. Bijnens, E. Pallante, J. Prades, Nucl. Phys. B626, 410 (2002), hep-ph/0112255

520. J. Prades, Nucl. Phys. Proc. Suppl. 181-182, 15 (2008), 0806.2250

521. J. Bijnens, J. Prades, Mod. Phys. Lett. A22, 767 (2007), hep-ph/0702170

522. J. Bijnens, J. Prades, Acta Phys. Polon. B38, 2819 (2007), hep-ph/0701240

523. E. De Rafael, Nucl. Phys. Proc. Suppl. 186, 211 (2009), 0809.3085

524. D.W. Hertzog, J.P. Miller, E. de Rafael, B. Lee Roberts, D. Stockinger (2007), 0705.4617

525. F. Jegerlehner, Lect. Notes Phys. 745, 9 (2008)

526. E. de Rafael, Phys. Lett. B322, 239 (1994), hep-ph/9311316

527. J. Bijnens, E. Gamiz, E. Lipartia, J. Prades, JHEP 04, 055 (2003), hep-ph/0304222

528. A.E. Dorokhov, W. Broniowski, Phys. Rev. D78, 073011 (2008), 0805.0760

529. M.J. Ramsey-Musolf, M.B. Wise, Phys. Rev. Lett. 89, 041601 (2002), hep-ph/0201297

530. K. Kampf, M. Knecht, J. Novotny, Eur. Phys. J. C46, 191 (2006), hep-ph/0510021

531. K. Kampf, B. Moussallam, Phys. Rev. D79, 076005 (2009), 0901.4688

532. K. Kampf (2009), 0905.0585

533. A.E. Dorokhov (2009), 0905.4577

534. A.E. Dorokhov (2009), 0912.5278

535. H.J. Behrend et al. (CELLO), Z. Phys. C49, 401 (1991)

536. P. Jean et al., Astron. Astrophys. 407, L55 (2003), astro-ph/0309484

537. O. Adriani et al. (PAMELA), Nature 458, 607 (2009), 0810.4995

538. J. Chang et al., Nature 456, 362 (2008)

539. A.A. Abdo et al. (Fermi LAT), Phys. Rev. Lett. 102, 181101 (2009), 0905.0025

540. F. Aharonian et al. (H.E.S.S.), Phys. Rev. Lett. 101, 261104 (2008), 0811.3894

541. F. Aharonian et al. (H.E.S.S), Astron. Astrophys. 508, 561 (2009), 0905.0105

542. R. Bernabei et al., Int. J. Mod. Phys. D13, 2127 (2004), astro-ph/0501412

543. R. Bernabei et al. (DAMA), Eur. Phys. J. C56, 333 (2008), 0804.2741

544. M. Pospelov, A. Ritz, M.B. Voloshin, Phys. Lett. B662, 53 (2008), 0711.4866

545. N. Arkani-Hamed, D.P. Finkbeiner, T.R. Slatyer, N. Weiner, Phys. Rev. D79, 015014 (2009), 0810.0713

546. D.S.M. Alves, S.R. Behbahani, P. Schuster, J.G. Wacker (2009), 0903.3945

547. M. Pospelov, A. Ritz, Phys. Lett. B671, 391 (2009), 0810.1502

548. J. Hisano, S. Matsumoto, M.M. Nojiri, Phys. Rev. Lett. 92, 031303 (2004), hep-ph/0307216

549. M. Cirelli, M. Kadastik, M. Raidal, A. Strumia, Nucl. Phys. B813, 1 (2009), 0809.2409

550. J. March-Russell, S.M. West, D. Cumberbatch, D. Hooper, JHEP 07, 058 (2008), 0801.3440

551. I. Cholis, G. Dobler, D.P. Finkbeiner, L. Goodenough, N. Weiner, Phys. Rev. D80, 123518 (2009), 0811.3641
552. I. Cholis, D.P. Finkbeiner, L. Goodenough, N. Weiner, JCAP 0912, 007 (2009), 0810.5344

553. N. Arkani-Hamed, N. Weiner, JHEP 12, 104 (2008), 0810.0714

554. P. Fayet, Phys. Lett. B95, 285 (1980)

555. P. Fayet, Nucl. Phys. B187, 184 (1981)

556. B. Holdom, Phys. Lett. B178, 65 (1986)

557. D. Tucker-Smith, N. Weiner, Phys. Rev. D64, 043502 (2001), hep-ph/0101138

558. S. Chang, G.D. Kribs, D. Tucker-Smith, N. Weiner, Phys. Rev. D79, 043513 (2009), 0807.2250

559. B. Batell, M. Pospelov, A. Ritz, Phys. Rev. D79, 115008 (2009), 0903.0363

560. R. Essig, P. Schuster, N. Toro, Phys. Rev. D80, 015003 (2009), 0903.3941

561. M. Reece, L.T. Wang, JHEP 07, 051 (2009), 0904.1743

562. F. Bossi (2009), 0904.3815

563. N. Borodatchenkova, D. Choudhury, M. Drees, Phys. Rev. Lett. 96, 141802 (2006), hep-ph/0510147

564. P.F. Yin, J. Liu, S.h. Zhu, Phys. Lett. B679, 362 (2009), 0904.4644

565. J.D. Bjorken, R. Essig, P. Schuster, N. Toro, Phys. Rev. D80, 075018 (2009), 0906.0580

566. B. Batell, M. Pospelov, A. Ritz (2009), 0906.5614

567. R. Essig, P. Schuster, N. Toro, B. Wojtsekhowski (2010), 1001.2557

568. M. Freytsis, G. Ovanesyan, J. Thaler, JHEP 01, 111 (2010), 0909.2862

569. M.J. Strassler, K.M. Zurek, Phys. Lett. B651, 374 (2007), hep-ph/0604261

570. P. Schuster, N. Toro, I. Yavin, Phys. Rev. D81, 016002 (2010), 0910.1602

571. M.J. Strassler (2006), hep-ph/0607160

572. T. Han, Z. Si, K.M. Zurek, M.J. Strassler, JHEP 07, 008 (2008), 0712. 2041

573. M.J. Strassler (2008), 0801.0629

574. M. Baumgart, C. Cheung, J.T. Ruderman, L.T. Wang, I. Yavin, JHEP 04, 014 (2009), 0901.0283

575. J.F. Gunion, D. Hooper, B. McElrath, Phys. Rev. D73, 015011 (2006), hep-ph/0509024

576. B. McElrath, Phys. Rev. D72, 103508 (2005), hep-ph/0506151

577. R. Dermisek, J.F. Gunion, B. McElrath, Phys. Rev. D76, 051105 (2007), hep-ph/0612031

578. J. Galloway, B. McElrath, J. McRaven, Phys. Lett. B670, 363 (2009), 0807.2657

579. M. Pospelov, Phys. Rev. D80, 095002 (2009), 0811.1030

580. C. Boehm, P. Fayet, Nucl. Phys. B683, 219 (2004), hep-ph/0305261

581. B. Aubert et al. (BaBar) (2009), 0908.2821 